\title{
Non-equilibrium structural Dynamics of incommensurate Charge-Density Waves
}

Diffractive Probing with a micron-scale ultrafast Electron Gun

\section{Dissertation}

zur Erlangung des mathematisch-naturwissenschaftlichen Doktorgrades

"Doctor rerum naturalium"

der Georg-August-Universität Göttingen

im Promotionsprogramm ProPhys

der Georg-August University School of Science (GAUSS)

\author{
vorgelegt von \\ Herrn Gero Storeck \\ aus Frankfurt am Main
}

Göttingen, 2020 
Betreuungsausschuss

Prof. Dr. Claus Ropers, IV. Physikalisches Institut

Prof. Dr. Vasily Moshnyaga, I. Physikalisches Institut

Mitglieder der Prüfungskommission

Referent: Prof. Dr. Claus Ropers, IV. Physikalisches Institut

Korreferent: Prof. Dr. Stefan Mathias. I. Physikalisches Institut

Weitere Mitglieder der Prüfungskommission

Prof. Dr. Vasily Moshnyaga, I. Physikalisches Institut

Prof. Dr. Fabian Heidrich-Meisner, Institut für Theoretische Physik

Prof. Dr. Steffen Schumann, Institut für Theoretische Physik

Prof. Dr. Tim Salditt, Institut für Röntgenphysik

Tag der mündlichen Prüfung: 12. Juni 2020 


\section{Abstract}

In recent years, charge-density wave (CDW) systems have been studied extensively, as they provide a diverse testing field for basic concepts in electron-phonon coupling, electron correlation, and structural phase transitions. In particular, time-resolved techniques have participated in that process, disentangling the dynamics of the various degrees of freedoms in such complex materials. As a recently developed pump-probe technique, ultrafast low-energy electron diffraction provides complementary insight into the CDW-coupled structural dynamics at the surface. This cumulative thesis covers the investigation of the incommensurate CDWs phases in layered tantalum disulfide, employing a new miniaturized electron gun in the ULEED setup.

In a first study, the design and fabrication process of the miniaturized electron gun are described. Finite element modeling supports the design process and provides helpful insight into the performance of the device and estimates for voltages as well as pulse duration. Photolithography and focused-ion-beam etching were used for building a contact support and the gun assembly, including the nanotip emitter, lens electrodes and the shielding. The pulse duration and transverse beam quality were extracted using the transient electric field effect at a copper grid and static diffraction patterns, respectively.

In a second study, the structural dynamics in the incommensurate and nearly commensurate CDW phase of tantalum disulfide were investigated employing 1 ps temporal resolution. The diffraction intensities of main lattice spots and CDW satellites, as well as the diffuse background, indicate a multi-step relaxation process. The comparison of different groups of diffraction spots allowed to correct for the phonon-related reductions, yielding the $\mathrm{CDW}$-associated periodic lattice distortion (PLD). The persistent reduction of the PLD amplitude and fluence-dependent relaxation cycles reveal a structural non-equilibrium situation exhibiting time constants exceeding typical phonon equilibration times. This is discussed in the context of hot populations of CDW excitation modes. Satellite spot broadening at the highest fluence points to the creation of CDW dislocation defects. 



\section{Contents}

1 Introduction 1

1.1 Theoretical Framework of Charge-Density Waves . . . . . . . . . . . . 5

1.1.1 Peierls Transition . . . . . . . . . . . . . . . . . 5 5

1.1.2 Static Electronic Susceptibility . . . . . . . . . . . . . . . . 6

1.1.3 Peierls-Fröhlich model . . . . . . . . . . . . . . . . . 8

1.1.4 Stability Criterion $\ldots \ldots \ldots \ldots . \ldots \ldots$

1.1.5 Fluctuation Effects . . . . . . . . . . . . . . . 15

1.2 Aspects of Low-energy electron diffraction . . . . . . . . . . . . . . . . 25

1.2.1 Experimental LEED Setup . . . . . . . . . . . . . . . . . . 27

1.2.2 Instrumental Response Function . . . . . . . . . . . . . . . . 28

1.2 .3 Scattering Theory . . . . . . . . . . . . . . . . . . . . . . 29

1.3 The layered Compound $1 T-\mathrm{TaS}_{2} \ldots \ldots \ldots \ldots \ldots \ldots$

1.3.1 CDW States . . . . . . . . . . . . . . . . . . . . . . . . 44

1.3.2 Electronic and Phonon Band Structures _ . . . . . . . . . . . . . . 48

1.3.3 Previous time-resolved Experiments . . . . . . . . . . . . . . . . . 50

2 Nanotip-based photoelectron Microgun $\quad 55$

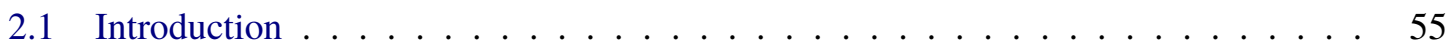

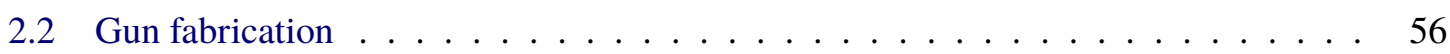

2.3 Numerical simulations . . . . . . . . . . . . . . . . . . . . . 57

2.4 Experimental Results . . . . . . . . . . . . . . . . . . . . . . 59

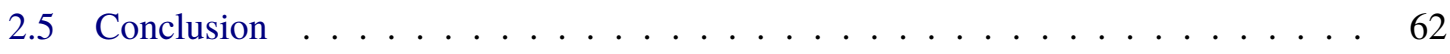

2.6 Acknowledgments . . . . . . . . . . . . . . . . . 62

3 Structural Dynamics of incommensurate Charge-Density Waves 63

3.1 Introduction . . . . . . . . . . . . . . . . . . . . . . . . 64 
3.2 Materials System and Experimental Approach . . . . . . . . . . . . . . . . 65

3.3 Results and Analysis . . . . . . . . . . . . . . . . . . . 68

3.3.1 Amplitude Analysis based on Main Lattice Reflexes . . . . . . . . . . . . . 69

3.3.2 Amplitude Analysis based on Satellite Reflexes . . . . . . . . . . . . 71

3.3.3 Non-equilibrium dynamics of the amplitude . . . . . . . . . . . . 73

3.4 Discussion . . . . . . . . . . . . . . . . . . . . . . . . . . 75

3.5 Conclusions . . . . . . . . . . . . . . . . . . . . . . . 77

3.6 Appendix . . . . . . . . . . . . . . . . . . . . . . . 78

3.6.1 Methods ....................... 78

3.6.2 Data for nearly commensurate $(\mathrm{NC})$ phase . . . . . . . . . . . . . 81

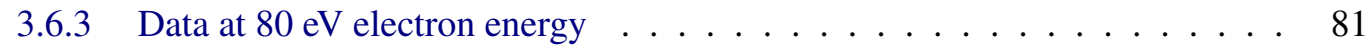

3.6.4 Relaxation cycles for main peaks $\left(\begin{array}{ll}-1 & 1\end{array}\right),\left(\begin{array}{ll}0 & 1\end{array}\right)$ and $\left(\begin{array}{llll}1 & -1\end{array}\right) \ldots \ldots 1$

3.6.5 Impact of CDW defects on peak width . . . . . . . . . . . 81

3.6.6 Dynamical LEED computation . . . . . . . . . . . . . . . . . . . . . . . 84

4 Discussion and Conclusions $\quad 87$

4.1 Summary of Publications . . . . . . . . . . . . . . . . . . . . . . 87

4.2 Improving the Electron Source $\ldots \ldots \ldots \ldots$. . . . . . . . . . . 88

4.3 CDW-related Prospects . . . . . . . . . . . . . . . . . . . . 95

4.4 Concluding Remarks $\ldots \ldots \ldots \ldots$

A Symbols and Abbreviations $\quad 99$

B NC-IC Spot Broadening $\quad 103$

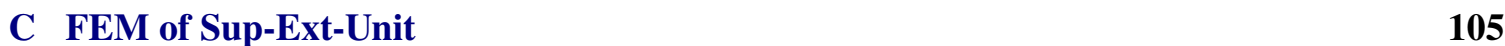

$\begin{array}{ll}\text { D Electron Gun Holder } & 107\end{array}$

$\begin{array}{ll}\text { E Diffraction Image Processing } & 109\end{array}$

$\begin{array}{ll}\text { Bibliography } & 115\end{array}$

$\begin{array}{ll}\text { Acknowledgements } & 145\end{array}$ 


\section{1 | Introduction}

In nature, most systems surrounding us, like the earth's atmosphere or all forms of life, are dynamic and therefore not in equilibrium. They exhibit energy and matter fluxes on a time and length scale that are typical for the specific system. Many of these dynamic processes that affect our daily lives take place within seconds, days, weeks or years. They can be grasped with our senses and we have developed an intuitive understanding. Nevertheless, we know today that there are much faster dynamics outside our perception, especially at the atomic level. In solids, non-equilibrium dynamics typically occur so rapidly and in such a small volume that they are beyond our normal intuition. The typical scales span over many orders of magnitude, ranging in time from atto- to nanoseconds and in space from micrometers down to picometers. To access these kinds of processes, probes featuring high spatio-temporal resolution are essential.

With the advent of femtosecond lasers, ultrafast light-triggered dynamics became possible, and an entire new field opened up aiming to study dynamic processes in materials systems across many disciplines, including physics, chemistry and biology. This branch of science seeks to study not only the initial and the final state of the process, but also to observe the intermediate states which allow insight into energy exchange, as well as reaction and transformation pathways. While initially the probing of materials was mainly realized with ultrashort light pulses [1], later on also ultrashort x-ray and electron pulses became available and widened the range of accessible observables [2]. More specifically, these probes added a complementary approach and paved the way to study non-equilibrium structural dynamics. Consequently, solids have been identified as the host of a rich set of non-equilibrium phenomena, such as non-thermal melting [3-6], meta-stable states [7, 8], characteristic phase excitations [9-12] or the dynamics in the creation and annihilation of topological defects $[13,14]$. Besides the scientific appeal, the discovery and skilful use of new materials and material properties are of crucial importance and guarantee future technological progress. A particularly interesting example is a new photo-induced non-equilibrium state that was suggested to serve a novel kind of non-volatile all-electronic ultrafast cryo-memory device [7, 15].

In ultrafast science, a common experimental approach is to deposit a high portion of energy 
in a small sample volume by intense laser illumination and to follow the subsequent energy flow cascading through the multiple degrees of freedom in the system by probing with a second pulse. In order to study different kinds of dynamical properties, many ultrafast probes have been developed in the past decades, such as ultrafast optical $[16,17]$ and photoemission spectroscopy $[18,19]$ for accessing electronic relaxation, ultrafast implementations of x-ray [20-22] and electron diffraction $[5,23,24]$ for the analysis of structural dynamics, or time-resolved magneto-optical pump-probe techniques for transient magnetic changes in the system [9].

The dimensionality of a materials system plays a crucial role as it controls the importance of interaction effects, like electron correlations or the electron-phonon coupling. Consequently, highly anisotropic structures in reduced dimensions often exhibit anomalous behavior [25], such as density waves or superconductivity [26]. By nature, a material's surface is two-dimensional and breaks the translational symmetry of an ideal periodic crystal, giving rise to many surface-specific phenomena, including altered band structures [19, 27], electronic surface states [28], surface reconstructions [29, 30] or topological states [31]. While this exemplifies the diversity of surface-specific effects, the class of low-dimensional systems also includes interfaces, adsorbates, thin layers or self-assembled nanostructures. Taken together, all of these systems constitute a vast playground for the study of new intriguing phenomena in reduced dimensions.

For many decades, it has been known [32] that low-dimensional metals can be unstable to a symmetry-lowering redistribution of charge, called charge-density wave (CDW), that is accompanied by a rearrangement of electronic bands and a periodic lattice distortion [26, 33]. The associated metal-insulator transition has a prominent role in solid state physics because it is not only relevant for electronic devices, but also because it is driven by a complex interplay of different electronic and structural degrees of freedom [34]. This interplay is responsible for phenomena such as strong renormalization in the phonon band structure (Kohn anomaly) as well as characteristic CDW fluctuation modes in amplitude and phase [26]. In particular, phase fluctuations in displacive incommensurate CDW systems have drawn much attention, as they were expected to 'slide' frictionless across the structure, possibly promoting a superconducting state [33, 35]. In ultrafast measurements [3638], however, the role of phase fluctuations is still under debate, as they remain very elusive and experimentally challenging to track.

Early studies on CDW materials include works on quasi-1D chain-like structures, such as the Krogmann's salt, transition-metal trichalcogenides (e.g., $\mathrm{NbSe}_{3}, \mathrm{TaS}_{3}, \mathrm{SmTe}_{3}$ [39]), transition metal bronzes $\left(\mathrm{A}_{0.3} \mathrm{MoO}_{3}\right.$ or $\mathrm{K}_{0.3} \mathrm{MoO}_{3}$ [40]) or the organic charge transfer compound TTF-TCNQ [41], as well as quasi-2D materials, like the layered transition-metal dichalcogenides (TMDCs) $\left(\mathrm{TaS}_{2}\right.$, $\mathrm{TiSe}_{2}$ ) [42]. More recently, charge-density waves on semiconductor and metal surfaces emerged as 
a new class of sample systems including self-assembled atomic indium chains on $\mathrm{Si}(111)$ [43] or $\mathrm{Cu}(001)$ [44], and bromine chains on $\operatorname{Pt}(110)$ [45]. In this work, we explore the structural dynamics of the layered material tantalum disulfide $\left(1 T-\mathrm{TaS}_{2}\right)$, one of the most prominent quasi-2D CDW systems that has a particularly rich phase diagram, including multiple CDW phases.

For the study of structural degrees of freedom at surfaces or thin layers, suitable diffraction techniques have been developed for which the scattering processes mainly take place in the first or first few layers. This mostly eliminates any parasitic signal from the bulk structure. Among these techniques are grazing incidence $\mathrm{x}$-ray, high-energy electron diffraction, and low-energy electron diffraction (LEED). The latter stands out as one of the most frequently used structural probes in surface science due to its high scattering efficiency, surface sensitivity, and its direct access to the structure's symmetry. Consequently, it would be highly beneficial to combine the strengths of LEED with a time-resolved approach in order to gain access to ultrafast structural phenomena in low-dimensional systems. In our group, we pursued this idea and recently developed ultrafast low-energy electron diffraction (ULEED) in transmission [46] as well as in backscattering geometry $[14,47-50]$.

The ULEED technique is based on a stroboscopic pump-probe approach in which a femtosecond optical pulse excites the sample out of equilibrium, while a subsequent ultrashort electron pulse probes the momentary state of the system. The entire ultrafast process can then be traced by changing the delay between these two pulses, recording snapshots for each configuration. For ultrafast electron techniques, a major challenge generally lies in reducing the electron pulse duration as it determines the achievable temporal resolution. Low-energy electron pulses are particularly susceptive to energetic broadening which complicates the realization of ultrashort pulses at the sample plane. Therefore, great effort is exerted into the development of suitable electron sources in order to generate electron pulses in the pico- and femtosecond regime. In the framework of this project, a new miniaturized electron gun was developed (see also Fig. D.1) that strongly enhanced the achievable temporal resolution by a factor of 50 (from about $50 \mathrm{ps}$ to $1 \mathrm{ps}$ ) and allowed ULEED to access a much broader range of ultrafast phenomena [14, 47, 49]. The first time-resolved study using this new gun is published in Ref. [14] highlighting its superior capabilities over the previous iteration, namely an equally high momentum resolution at significantly shorter electron pulse durations, and revealing the important role of CDW dislocation defects in the phase-ordering kinetics in the NC-IC transition of $1 T-\mathrm{TaS}_{2}$.

Outline The remainder of Chapter 1 covers the relevant background for the reprinted publications in the subsequent chapters. In the beginning, a review on the theoretical framework of charge-density 
waves is given. The chapter elucidates the emergence of the Peierls state and presents the common Peierls-Fröhlich model that in the one-dimensional case reproduces the most important features of CDW systems, such as a renormalized phonon dispersion, a gapped electronic spectrum as well as the charge-density modulation. The characteristic fluctuation modes of a CDW system and the effects of large phase fluctuations are briefly discussed. In the succeeding section, the benefits of low-energy electron scattering, the LEED technique itself and diffraction theory are introduced. The latter includes the discussion of the scattering amplitude, the real and reciprocal lattice, the Ewald construction in two dimensions and several detailed aspects affecting the diffracted signal. More specifically, it is shown that diffraction is sensitive to the PLD associated with collective excitations, defects, and thermal effects. The last section is a brief overview of the materials system $1 T-\mathrm{TaS}_{2}$ whose various CDW states, the electronic and the phonon band structure are briefly reviewed. Since this chapter has to cover many aspects from different fields, the reader will be referred to the appropriate literature for some details as indicated in the text.

Chapter 2 presents the development of a micron-scale ultrafast electron gun. The content of this chapter is published as Ref. [47], "Nanotip-based photoelectron microgun for ultrafast LEED". Here, we describe the fabrication process, present numerical studies supporting the gun performance, and characterize the low-energy electron pulses, yielding a duration of $1.3 \mathrm{ps}$ at an electron energy of $80 \mathrm{eV}$. We show first diffraction pictures of $1 T-\mathrm{TaS}_{2}$ in backscattering geometry using this electron gun.

While Chapter 2 shows the technological advances of the electron gun design, Chapter 3 presents a comprehensive study of the non-equilibrium dynamics in the NC- and IC phases of $1 T-\mathrm{TaS}_{2}$ using ULEED with 1ps temporal resolution without driving the phase transitions. The content of this paper is published in Ref. [49], "Structural Dynamics of incommensurate Charge-Density Waves tracked by Ultrafast Low-Energy Electron Diffraction". Here, we extract the transient mean amplitude of the periodic lattice distortion which shows a surprisingly long-lived, non-thermal suppression. We discuss this in the context of CDW fluctuation modes.

In Chapter 4, the key results of the two published works are summarized. Subsequently, we discuss ways of further technological improvement and outline possible future experiments on $1 T-\mathrm{TaS}_{2}$ or related material systems that could complement and deepen our understanding of the characteristic properties of CDW fluctuation modes. 


\subsection{Theoretical Framework of Charge-Density Waves}

A charge-density wave (CDW) is a type of a broken-symmetry state that leads to long-range order of the charge-density and the atomic distortion field. CDWs form preferably in low-dimensional metals in the presence of electron-phonon interactions. Long before physical manifestations were known, this idea was first promoted by Peierls [32] for 1d systems in which the metallic state is unstable when coupled to phonons at low temperatures. Interestingly, Peierls himself was not aware of the relevance for real physical systems, as he writes in his book [51]:

"This instability came to me as a complete surprise when I was tidying material for my book [32], and it took me a considerable time to convince myself that the argument was sound. It seemed of only academic significance, however, since there are no strictly one-dimensional systems in nature (and if there were, they would become disordered at any finite temperature). I therefore did not think it worth publishing the argument, beyond a brief remark in the book, [...]."

In theory, long-range order does not emerge in strictly one- or two-dimensional systems with shortrange interaction at finite temperatures since fluctuations of the order parameter play a major role $[26,52,53]$. Known as the Mermin-Wagner theorem [53], this statement, however, does not hold for so-called quasi-one- or quasi-two-dimensional systems. The term 'quasi' signifies that interactions and couplings, such as Coulomb forces between neighboring chains, Van-der-Waals forces between layers, or phonons are taken into account that ultimately introduce a $3 \mathrm{~d}$ character to the material.

The original literature on the theory of charge-density waves, dating from the 1970s, treats mostly one-dimensional systems. Since the treatment of $1 \mathrm{~d}$ cases cover the important features, we follow this line to understand the basic mechanism. Where possible or necessary, we widen the view to also cover $2 \mathrm{~d}$ or $3 \mathrm{~d}$ systems.

\subsubsection{Peierls Transition}

Peierls was the first to promote that a monoatomic chain with the lattice constant $a$ should be unstable when a periodic lattice distortion is introduced. Following his argument [32], we assume that each atom contributes one electron, leading to a half-filled band (Fig. 1.1 left). The band is half-filled because there are two electrons with opposite spin states in each energy state.

In the original configuration of the $1 \mathrm{~d}$ chain, the reciprocal lattice constant is given by $a^{*}=2 \pi / a$. 
An equal displacement of every other atom introduces a new periodicity in the lattice that leads to degenerate states at $\boldsymbol{k}= \pm \boldsymbol{k}_{F}$ where $\boldsymbol{k}_{F}$ denotes the Fermi wave vector. The removal of the degeneracy via a weak interaction with the lattice leads to electronic gaps (see Fig. 1.1 right) and induces a metal-insulator transition. The occupied states at $\pm \boldsymbol{k}_{F}$ are lowered while the empty states are lifted in energy, resulting in a decrease of the total electronic energy contribution. The new reciprocal lattice vector now reads $a_{\mathrm{dist}}{ }^{*}=\pi / 2 a=k_{F}$. This new ground state, the Peierls state, forms if the total energy of the system, including the contribution of the static lattice distortion due to Coulomb repulsion, is lower than in the initial state (see Section 1.1.4).
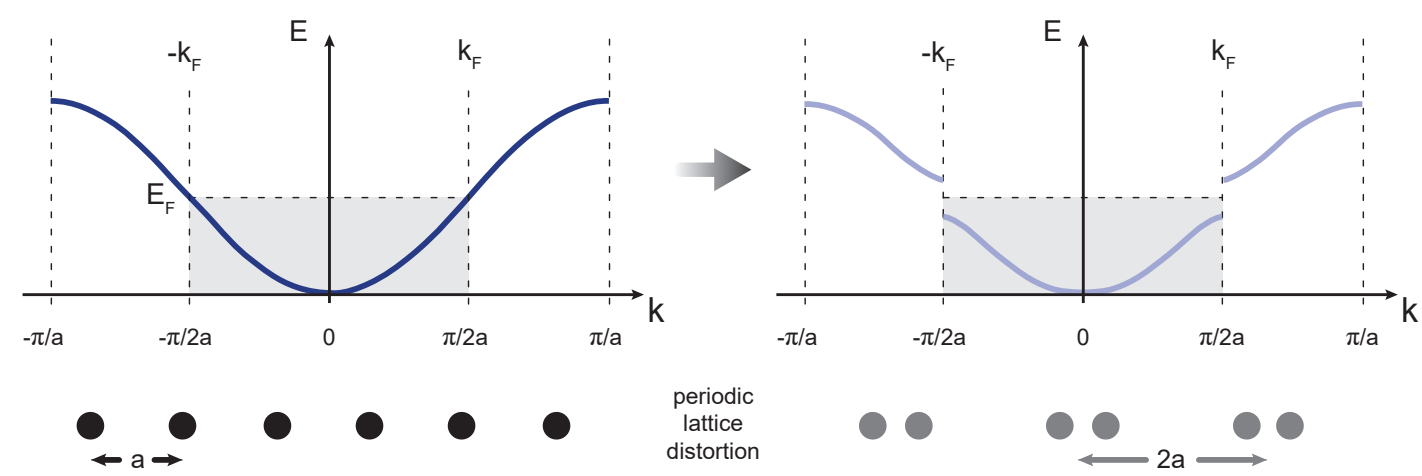

Figure 1.1: Original Peierls mechanism. A lattice distortion introduces a new periodicity and alters the the electronic band structure that forms CDW gaps at the wave vectors $\boldsymbol{k}= \pm \boldsymbol{k}_{F}$.

In the literature, the nomenclature of this state sometimes leads to confusion, as the term chargedensity wave state emphasizes the static modulation of the charge density. It is important to note, however, that a CDW is a coupled entity showing both a modulation of the charge and a periodic lattice distortion (PLD). Although in principle, low-energy electrons can show a sensitivity to valence electrons, the PLD of the CDW is probed by LEED in this work.

\subsubsection{Static Electronic Susceptibility}

For a deeper understanding of the emerging Peierls state, it is instructive to study the general properties of the conduction electrons in a metal, based on the model of an electron gas. Specifically, an important aspect is the response to an external potential, which changes dramatically for lower dimensions [26].

The response of an electron gas to a weak, spatially dependent external potential $\phi_{\mathrm{ext}}(\boldsymbol{r})$ is commonly studied in the context of linear response theory [26, 54]. The response function is generally temperature-dependent. In this theory, the Fourier components of the induced charge 
density are given by [26]

$$
\rho_{\text {ind }}(\boldsymbol{Q}, T)=\chi(\boldsymbol{Q}, T) \phi(\boldsymbol{Q}) .
$$

The function $\chi(Q, T)$ is the so-called static response or susceptibility of the system that defines how the electron system responds to the total potential $\phi(\boldsymbol{Q})$. The total potential also includes the induced potential that has to be taken into account in the self-consistent treatment [54]. The susceptibility of the electron gas in $d$ dimensions reads

$$
\chi(\boldsymbol{Q}, T)=\int \frac{d k}{2 \pi^{d}} \frac{f\left(\epsilon_{\boldsymbol{k}}\right)-f\left(\epsilon_{\boldsymbol{k}+\boldsymbol{Q}}\right)}{\epsilon_{\boldsymbol{k}}-\epsilon_{\boldsymbol{k}+\boldsymbol{Q}}} .
$$

Here, $\epsilon_{k}$ denotes the energy of a state with momentum $\boldsymbol{k}$ and $f\left(\epsilon_{\boldsymbol{k}}\right)$ is the Fermi function given by

$$
f\left(\epsilon_{k}, T\right)=\frac{1}{\exp \left(\left(\epsilon_{k}-\mu\right) / k_{B} T\right)+1}
$$

with the chemical potential $\mu$ and the Boltzmann constant $k_{B}$. For zero temperature, $\chi(Q, T)$ can be analytically evaluated near the wavevector $2 k_{F}$ and, in the one- and two dimensional case, yield [55-57]

$$
\begin{aligned}
& \chi_{1 \mathrm{~d}}(Q, T=0) \propto \ln \left(\frac{Q+2 k_{F}}{Q-2 k_{F}}\right) \\
& \chi_{2 \mathrm{~d}}(Q, T=0) \propto \begin{cases}1 & \text { for } Q<k_{F} \\
1-\sqrt{1-\left(2 k_{F} / Q\right)^{2}} & \text { for } Q>k_{F},\end{cases}
\end{aligned}
$$

where $n$ denotes the electron density and $\hbar$ the reduced Planck constant.

In comparison to the results of a higher-dimensional free electron gas (displayed in Fig. 1.2), the $1 \mathrm{~d}$ response function exhibits a drastically different behavior with a logarithmic divergence at $\boldsymbol{Q}=2 \boldsymbol{k}_{F}$. in Equation 1.2, the terms with a large contribution to $\chi$ are the ones obeying the condition $\boldsymbol{Q} \approx \pm 2 \boldsymbol{k}_{F}$ and $\boldsymbol{k} \approx \mp \boldsymbol{k}_{F}$. More specifically, pairs of states, one empty and another occupied, are connected by the same wavevector $\boldsymbol{Q}$ and differ negligibly in energy. This so-called Fermi surface nesting leads to a diverging response function [58] and a spatially varying induced charge density modulation according to equation 1.1 [59].

The qualitative difference of the response function for each dimensionality (Fig. 1.2b) stems from differently shaped Fermi surface topologies (Fig. 1.2a). Hence, the efficiency of Fermi nesting depends on the amount of Fermi surface that can be mapped onto itself by the same momentum vector $\boldsymbol{Q}$ (see Fig. 1.2a). In 1d, the Fermi surface consists of two points resulting in a perfect nesting and a divergence in $\chi$, whereas for $2 \mathrm{~d}$ and $3 \mathrm{~d}$, only a small fraction is connected, attenuating the divergence in the response function. Yet, strongly anisotropic or 'star-shaped' Fermi surfaces (Fig. 1.2a bottom) can also yield parallel segments that allow for an instability to occur. 
a

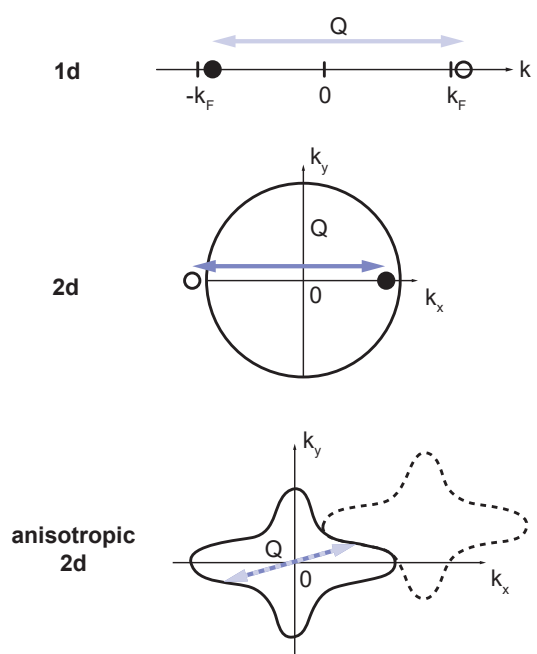

b

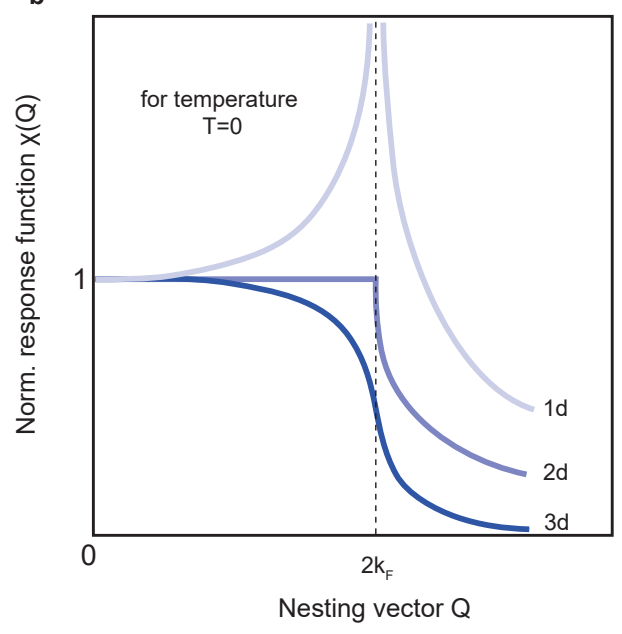

Figure 1.2: Fermi surface nesting. (a) Fermi surfaces for a $1 d, 2 d$ and an anisotropic $2 d$ case. Arrows indicate nesting vectors that connect parallel elements on the Fermi surface. (b) Response function in 1d, $2 \mathrm{~d}$ and $3 \mathrm{~d}$ systems for zero temperature. Dimensionality affects the strength of the instability at $Q=2 k_{F}$. Adapted from Ref. [55].

Generally, the response function $\chi(\boldsymbol{Q}, T)$ is temperature-dependent, as it is defined through the Fermi distribution $f(\epsilon, T)$. Thus, a finite temperature smears the Fermi edge and attenuates the response function $\chi(\boldsymbol{Q}, T)$. In $1 \mathrm{~d}, \chi\left(2 \boldsymbol{k}_{F}, T\right)$ is given by

$$
\chi\left(2 \boldsymbol{k}_{F}, T\right)=-e^{2} n\left(\epsilon_{F}\right) \ln \left(\frac{1.14 \epsilon_{0}}{k_{B} T}\right),
$$

where $e$ is the electron charge, $n\left(\epsilon_{F}\right)$ the electron density at the Fermi energy, $\epsilon_{0}$ is the dielectric constant and $k_{B}$ is the Boltzmann constant. Hence, for $\boldsymbol{Q} \rightarrow 2 \boldsymbol{k}_{F}$, the susceptibility diverges logarithmically with decreasing temperature, leading to an induced charge density modulation $\rho\left(2 \boldsymbol{k}_{F}\right)$.

At this point, we have not explicitly specified the external potential that could drive the instability. The Peierls picture suggests that the conduction electrons become unstable under a perturbation caused by a periodic distortion of the lattice. This line of argument is covered in the following sections.

\subsubsection{Peierls-Fröhlich model}

In the previous section, we have seen that the electron gas is unstable under the perturbation of a periodic potential. Here, we introduce a microscopic model with an explicit electron-phonon 
coupling that mimics the interaction with a lattice. Hence, this extended model also covers the structural change of the lattice that is associated with the Peierls transition.

Electron-phonon coupling has been studied extensively, both experimentally and theoretically, due to its ubiquity in condensed matter physics [60-62], in particular in the context of superconductivity. In the discussion of charge-density waves, a frequently encountered model is the so-called Fröhlich Hamiltonian [33]:

$$
H=H_{0}+H_{\text {el-ph}} \text {. }
$$

It consists of two parts, namely an unperturbed part $H_{0}$ describing electron quasiparticles and lattice vibrations (phonons), and the interaction Hamiltonian $H_{\mathrm{el}-\mathrm{ph}}$, considering a short-ranged electronphonon coupling. For the derivation, we refer to the Refs. [26, 55, 63]. In the formalism of second quantization [64], the unperturbed part is given by

$$
H_{0}=\sum_{\boldsymbol{k}} \epsilon_{\boldsymbol{k}} c_{\boldsymbol{k}}^{+} c_{\boldsymbol{k}}+\sum_{\boldsymbol{k}} \hbar \omega_{\boldsymbol{Q}} b_{\boldsymbol{Q}}^{+} b_{\boldsymbol{Q}}
$$

where the first term describes the electron quasiparticle gas by a sum of creation and annihilation operators $c_{\boldsymbol{k}}$ and $c_{\boldsymbol{k}}^{+}$, respectively, with dispersion $\epsilon_{\boldsymbol{k}}$ and wave vector $\boldsymbol{k}$. For simplicity, we omit the spin and consider a single band. The second term covers the quantized harmonic vibrations of the lattice with the corresponding bosonic ladder operators $b_{\boldsymbol{Q}}^{+}$and $b_{\boldsymbol{Q}}$ of a phonon mode with energy $\omega_{\boldsymbol{Q}}$ and wave vector $\boldsymbol{Q}$ [65]. For convenience, only a single longitudinally polarized acoustic phonon branch is considered. With the given notation, the interaction Hamiltonian for lowest-order coupling (Born approximation and small displacements of atoms [66]) between electrons and phonons reads

$$
\begin{aligned}
H_{\mathrm{el}-\mathrm{ph}} & =\sum_{\boldsymbol{k}, \boldsymbol{Q}} g_{\boldsymbol{k}, \boldsymbol{k}^{\prime}}\left(b_{-\boldsymbol{Q}}^{+}+b_{\boldsymbol{Q}}\right) c_{\boldsymbol{k}+\boldsymbol{Q}}^{+} c^{c_{k}} \\
\text { with } g_{\boldsymbol{k}, \boldsymbol{k}^{\prime}} & =i \sqrt{\frac{N \hbar}{2 M \omega_{\boldsymbol{Q}}}}\left|\boldsymbol{k}^{\prime}-\boldsymbol{k}\right| V_{\boldsymbol{k}-\boldsymbol{k}^{\prime},}
\end{aligned}
$$

where $g_{\boldsymbol{k}, \boldsymbol{k}^{\prime}}$ is the electron-phonon coupling constant that describes the probability amplitude for scattering an electron with momentum $\boldsymbol{k}$ to a state with momentum $\boldsymbol{k}^{\prime}=\boldsymbol{k} \pm \boldsymbol{Q}$ under the simultaneous absorption (emission) of a phonon with momentum $\boldsymbol{Q}(-\boldsymbol{Q})$. The quantities $M, N$ and $V_{\boldsymbol{k}-\boldsymbol{k}^{\prime}}$ are the atomic mass, the atom density and the single atom potential in Fourier space, respectively. The scattering can be visualized diagrammatically (see Fig. 1.3) and corresponds to the terms $b_{-\boldsymbol{Q}}^{+} c_{\boldsymbol{k}+\boldsymbol{Q}} c_{\boldsymbol{k}}$ and $b_{\boldsymbol{Q}} c_{\boldsymbol{k}+\boldsymbol{Q}}^{+} c_{\boldsymbol{k}}$ in the interaction Hamiltonian. The lattice displacement, in terms of the bosonic ladder operators, is given by

$$
u(\boldsymbol{x})=\sum_{\boldsymbol{Q}} \sqrt{\frac{\hbar}{2 N M \omega_{\boldsymbol{Q}}}}\left(b_{\boldsymbol{Q}}+b_{-\boldsymbol{Q}}^{+}\right) e^{i \boldsymbol{Q} \boldsymbol{x}} .
$$




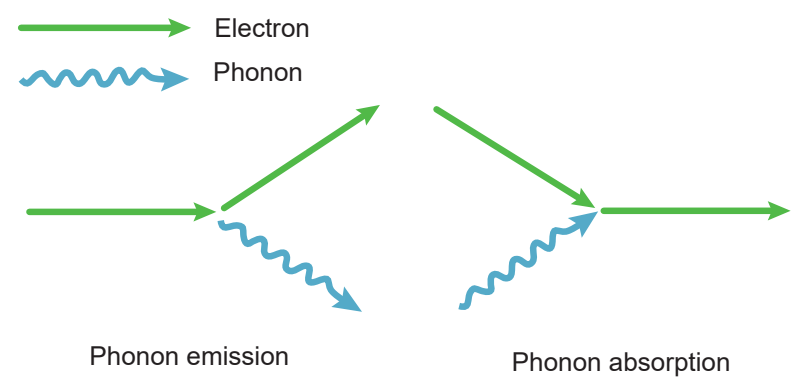

Figure 1.3: First order scattering processes between electrons and phonons in the Peierls-Fröhlich model. Adapted from Ref. [67].

\subsubsection{Kohn Anomaly}

Based on the Fröhlich Hamiltonian, we investigate the impact of electron-phonon interaction on the phonon dispersion relation. As an outcome, we will find a renormalized phonon dispersion at $\boldsymbol{Q}=2 \boldsymbol{k}_{F}$ called Kohn anomaly. In three dimensions, the change in energy correction is relatively small and can be calculated via second-order perturbation theory [54], whereas in quasi-onedimensional systems the correction to the energy can be significant resulting even in a vanishing phonon energy (giant Kohn anomaly) and a structural phase transition with a frozen-in CDW-coupled mode (see Fig. 1.4a). In the latter case, instead of low-order perturbation theory, a preferable theoretical description is a mean-field theory that treats the phonon system in the presence of a mean electronic density.

Following the derivation of the Kohn anomaly in Ref. [26], the essential idea is to determine the temporal evolution for the periodic lattice distortion $u(x)$ that leads to a simple equation of an harmonic oscillator. This can be done by evaluating the relevant commutators of the phonon operators $b_{\boldsymbol{Q}}$ and $b_{-\boldsymbol{Q}}^{+}$, and leads to the following equation

$$
\left(\frac{d}{d t}\right)^{2}\left(b_{\boldsymbol{Q}}+b_{\boldsymbol{Q}}^{+}\right)=-\left(\omega_{\boldsymbol{Q}}+\frac{2 g^{2} \omega_{\boldsymbol{Q}}}{M \hbar} \chi(Q, T)\right)\left(b_{\boldsymbol{Q}}+b_{\boldsymbol{Q}}^{+}\right),
$$

where $g$ is again the electron-phonon coupling taken to be constant and independent of $\boldsymbol{k}$ or $\boldsymbol{Q}$. In the derivation, the electron density $n_{\boldsymbol{Q}}$ emerging in the coupling term was replaced by its expectation value $\left\langle n_{\boldsymbol{Q}}\right\rangle$ (mean field) which is associated with the lattice deformation via the response function $\chi$. From equation 1.12, we can extract a renormalized phonon frequency

$$
\omega_{\mathrm{ren}, \boldsymbol{Q}}^{2}=\omega_{\boldsymbol{Q}}^{2}+\frac{2 g^{2} \omega_{\boldsymbol{Q}}}{M \hbar} \chi(\boldsymbol{Q}, T)
$$

As discussed above, the one-dimensional electron gas is unstable against a perturbation with wavevector $\boldsymbol{Q}=2 \boldsymbol{k}_{F}$ yielding a diverging susceptibility. Therefore, the phonon dispersion will 
be strongly lowered, or softened, in the vicinity of this wavevector where an optic mode starts to condensate [68]. Inserting the temperature-dependent expression given in Equation 1.6, the renormalized phonon frequency in $1 \mathrm{~d}$ is then given by

$$
\omega_{\mathrm{ren}, 2 \boldsymbol{k}_{F}}^{2}=\omega_{2 \boldsymbol{k}_{F}}^{2}-\frac{2 g^{2} n\left(\epsilon_{\boldsymbol{k}}\right) \omega_{2 \boldsymbol{k}_{F}}}{\hbar} \ln \left(\frac{1.14 \epsilon_{0}}{k_{B} T}\right)
$$

Figure $1.4 \mathrm{~b}$ shows the phonon dispersion $\omega_{\mathrm{ren}, 2 \boldsymbol{k}_{F}}^{2}$ relation for various temperatures as determined by equation 1.14. At the transition temperature $T_{c}$ and in a one-dimensional system, the renormalized phonon frequency vanishes due to a diverging response function $\chi$, and the system undergoes a structural phase transition, which is called Peierls transition. In higher dimensions shown in Fig. 1.4a, the dependence on $\chi(\boldsymbol{Q}, T)$ is weaker and the phonon softening less prominent. For a weak electron-phonon coupling $g$, the renormalized phonon frequency therefore remains finite, and no phase transition occurs. Consequently, a Peierls state in quasi-2d materials is favored by a non-zero electron-phonon coupling and an efficient Fermi nesting.

a

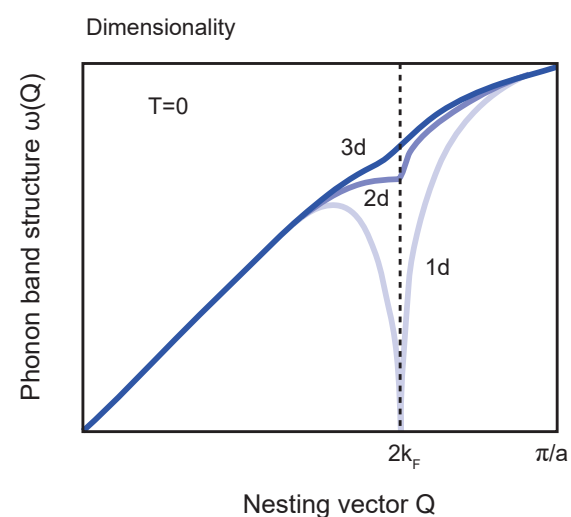

b

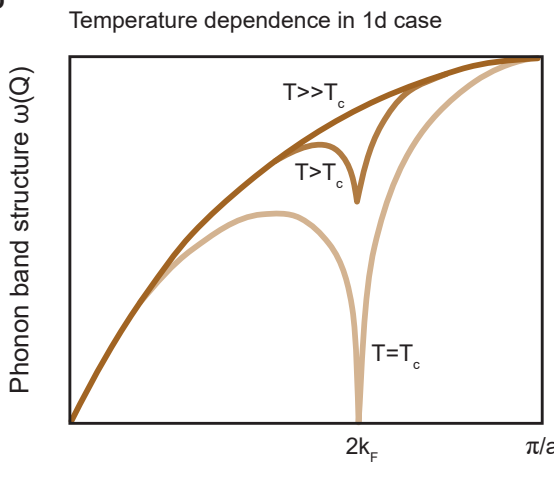

Nesting vector $Q$

Figure 1.4: Phonon softening. (a) Dimensionality dictates the strength of the Kohn anomaly. (b) Above $T_{c}$, phonon softening is an indicator for the Kohn anomaly. Adapted from Ref. [26]

Furthermore, below the transition temperature, zero-energy $2 \boldsymbol{k}_{F}$-phonons condensate in a macroscopic number, motivating the definition of an order parameter $\Delta$ that is based on the expectation value of the phonon operators. Borrowed from the theory of superfluidity [54], the expectation values behave as complex numbers, vanish above $T_{c}$, and have a finite value below. The complex order parameter $\Delta$ is described by

$$
\Delta=|\Delta| e^{i \varphi}=g_{2 \boldsymbol{k}_{F}}\left(\left\langle b_{2 \boldsymbol{k}_{F}}\right\rangle+\left\langle b_{-2 \boldsymbol{k}_{F}}^{+}\right\rangle\right),
$$

where $\Delta \in \mathbb{R}$ denotes the amplitude and $\varphi \in \mathbb{R}$ the phase. 
Accordingly, the expectation value of the lattice displacement $\langle u(\boldsymbol{x})\rangle$ (PLD), an observable in our experiment, takes the form

$$
\begin{aligned}
\langle u(\boldsymbol{x})\rangle & =\sqrt{\frac{\hbar}{2 N M \omega_{\boldsymbol{Q}}}}\left[\left(\left\langle b_{2 \boldsymbol{k}_{F}}\right\rangle+\left\langle b_{-2 \boldsymbol{k}_{F}}^{+}\right\rangle\right) e^{i 2 \boldsymbol{k}_{F} \boldsymbol{x}}+c . c\right] \\
& =\underbrace{\sqrt{\frac{\hbar}{2 N M \omega_{\boldsymbol{Q}}}} \frac{2|\Delta|}{g}}_{\Delta u} \cos \left(2 \boldsymbol{k}_{F} \boldsymbol{x}+\varphi\right) .
\end{aligned}
$$

Finite values of $\left\langle b_{ \pm 2 \boldsymbol{k}_{F}}\right\rangle$ lead to atom displacements away from their equilibrium positions and a static PLD with a $2 \boldsymbol{k}_{F}$-periodicity. In other words, the presence of a PLD is a direct measure of the CDW-order in the system. The complex nature of the order parameter will lead to characteristic collective excitations in the system, shown below in the context of the Ginzburg-Landau theory.

\subsubsection{Electronic Band Structure}

We now switch our point of view and examine the electronic spectrum in the presence of a mean distortion field. The Fröhlich Hamiltonian in this phonon mean-field approximation takes the form

$$
H=\sum_{\boldsymbol{k}} \epsilon_{\boldsymbol{k}} c^{+} c_{\boldsymbol{k}}+\sum_{\boldsymbol{Q}} \hbar \omega\left\langle b_{\boldsymbol{Q}}^{+} b_{\boldsymbol{Q}}\right\rangle+\sum_{\boldsymbol{k}, \boldsymbol{Q}} g_{\boldsymbol{Q}}\left\langle b_{-\boldsymbol{Q}}^{+}+b_{\boldsymbol{Q}}\right\rangle c_{\boldsymbol{k}+\boldsymbol{Q}}^{+} c_{\boldsymbol{k}}
$$

In the nearly-free electron approximation, the Hamiltonian can be diagonalized via a canonical transformation [63] for the relevant $2 \boldsymbol{k}_{F}$-phonon modes. We omit the detailed derivation and state the result for the electronic dispersion $[26,54]$

$$
E_{k}=\epsilon_{F}+\operatorname{sign}\left(\boldsymbol{k}-\boldsymbol{k}_{F}\right) \sqrt{\hbar^{2} v_{F}^{2}\left(\boldsymbol{k}-\boldsymbol{k}_{F}\right)^{2}+\Delta^{2}},
$$

where $v_{F}$ is the Fermi velocity. The dispersion $E_{k}$ exhibits single-particle gaps at the modes $\boldsymbol{k}= \pm \boldsymbol{k}_{F}$ of the size $2|\Delta|$ transforming the prior metal state into an Peierls insulator if the condensate does not contribute to the electric conductivity. Consequently, the amplitude of the order parameter can be experimentally accessed, for example, via spectroscopic techniques measuring the band structure of the material and identifying CDW gaps.

Moreover, the charge density $\rho$ in the Peierls state can be determined utilizing the new ground state wave functions yielding

$$
\rho(\boldsymbol{x})=\rho_{0}\left[1+\frac{\hbar \omega_{2 \boldsymbol{k}_{F}}}{g^{2} n\left(\epsilon_{F}\right)} \frac{\Delta}{\hbar v_{F} \boldsymbol{k}_{F}} \cos \left(2 \boldsymbol{k}_{F} \boldsymbol{x}+\varphi\right)\right],
$$

where $\rho_{0}$ is the constant electron density in the metallic state. The periodic form of the charge density motivates the name charge-density wave, already introduced above. 
Figure 1.5 shows the charge density modulation, the periodic lattice distortion and the electronic dispersion relation, in the metallic and the Peierls state for a one-dimensional chain. For illustration purposes, the band is half-filled which, however, represents a special case since the chain dimerizes with a periodicity $\lambda_{\mathrm{PLD}}=\pi / \boldsymbol{k}_{F}=\pi / 2 a$.

Generally, a system with two coexisting periodicities is classified as commensurate or incommensurate corresponding to a rational or irrational ratio of periodicities. In this example, the two periodicities are associated with the regular lattice of the chain and the distortion field. Hence, the dimerized chain is commensurate while it is incommensurate for an irrational filling. In the latter case, the total energy of the Peierls state is independent of the order parameter phase $\varphi$. For arbitrary values of $\varphi$, the charge-density wave can adiabatically go from one energy state to another and has the freedom to slide along the chain resulting in sliding modes of the CDW state. This notion originally stems from magnetic and compositional incommensurate structures [68] which represent a different type of incommensurateness compared to the $\mathrm{CDW}$-induced displacive character studied in this work $[69,70]$. The additional degree of freedom has important consequences for the collective excitation spectrum as well as for the electronic transport behavior [71].

a

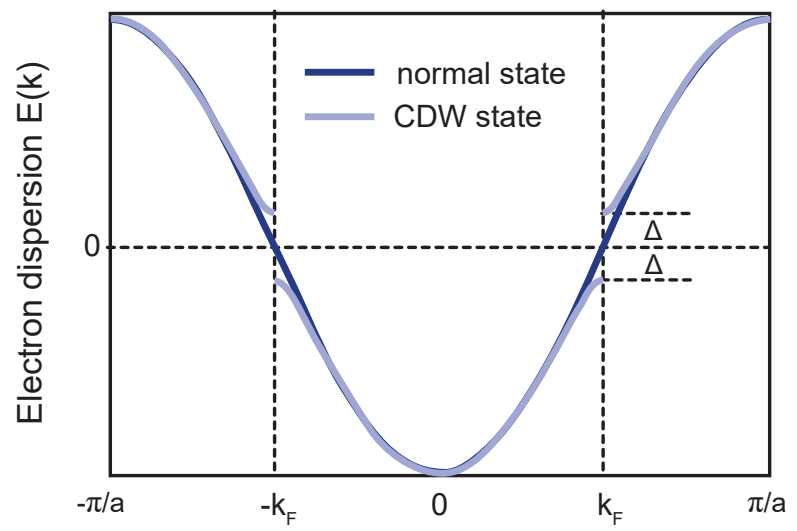

b

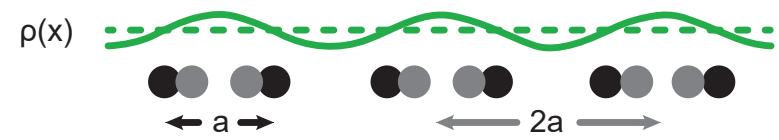

Figure 1.5: CDW as an coupled object. (a) In the mean field of lattice modes, the electronic spectrum is altered resulting in CDW gaps at $\pm \boldsymbol{k}_{F}$. (b) The charge density (green) and atomic positions (black and grey dots) are periodically modulated. Adapted from Ref. [26]. 


\subsubsection{Stability Criterion}

A metal transforms to a Peierls insulator when its ground state lies energetically lower than the metallic state. To this end, we compare the energy gain due to the opening of gaps in the band structure with the energy cost necessary to distort the lattice [72]. The lattice strain energy for a (mean) lattice distortion $u_{\boldsymbol{Q}}$ with frequency $\omega_{\boldsymbol{Q}}$ and momentum $\boldsymbol{Q}$ is given by

$$
\Delta E_{\mathrm{lat}}=\frac{1}{2} M \omega_{\boldsymbol{Q}}^{2} u_{\boldsymbol{Q}}^{2}
$$

The energy gained from the electron system is expressed by

$$
\Delta E_{\mathrm{band}}=-\left|v_{Q}\right|^{2} \chi(\boldsymbol{Q})
$$

with

$$
v_{\boldsymbol{Q}}=\frac{g_{\boldsymbol{Q}} u_{\boldsymbol{Q}} \sqrt{2 M \omega_{\boldsymbol{Q}}}}{\hbar},
$$

where $v_{\boldsymbol{Q}}$ is the effective potential, set up by the lattice distortion. The CDW ground state will be stable if

$$
\Delta E_{\mathrm{lat}}+\Delta E_{\mathrm{band}}<0
$$

for a specific phonon mode $\boldsymbol{Q}$ which leads to the criterion

$$
\frac{4 g_{\boldsymbol{Q}}^{2}}{\hbar \omega_{\boldsymbol{Q}}}>\frac{1}{\chi(\boldsymbol{Q})} .
$$

The Peierls state will be promoted in the vicinity of an electronic instability and for a large electronphonon coupling constant $g_{\boldsymbol{Q}}$.

A theoretical study by Johannes and Mazin [73] investigated the role of Fermi nesting in $\mathrm{NbSe}_{2}, \mathrm{TaSe}_{2}$, and $\mathrm{CeTe}_{2}$ and, pointed out that only few CDW transitions are true analogues of a Peierls instability since electronic instabilities are easily destroyed by even small deviations from perfect nesting conditions. Consequently, the strength and the $\boldsymbol{Q}$-dependence of the electron-phonon coupling $g_{\boldsymbol{Q}}$ have to be considered as an integral part in the formation of a CDW [25,73], in particular for quasi-2d systems in which CDW formation might not be rationalized purely by a simple Fermi nesting mechanism [74].

The characteristics of a CDW system can be quite diverse depending on the strength of the electron-phonon coupling. The weak coupling regime with small CDW gaps and displacements $\left(\Delta / \epsilon_{F} \ll 1\right.$ and $\left.\Delta u / a \ll 1\right)$ can be well understood in the above introduced Peierls-Fröhlich picture, while the electronic gaps and atomic displacements are typically larger in the strong coupling limit, and the spatial coherence length decreases due to larger fluctuations [75]. Additionally, nonlinear terms in the electron-phonon interaction have a considerable contribution and tend to 'lock in' the 
CDWs to the lattice [25]. For both limits, the energy gain is of electronic origin. However, for weak coupling, the gain is mostly around $\pm \boldsymbol{k}_{F}$ and is proportional to $\Delta^{2} \ln \Delta$, while for strong coupling the gain is spread over the entire Brillouin zone and is proportional to $\Delta$ [72]. Most real materials are situated in between these limiting regimes.

A further degree of complexity is introduced when electron-electron correlation becomes more important, usually for low temperatures. The above introduced Peierls-Fröhlich model (see Equation 1.10) neglects such an explicit electron-electron interaction term, but rather incorporates weak electron correlation effects. It enters implicitly trough the dispersion $\epsilon_{k}$ of non-interacting quasiparticles which are regarded as stationary solutions of band electrons in a perfect periodic lattice including the renormalization from Coulomb interactions [65].

In an expanded approach, Chan and Heine derived a more general stability criterion, also including the effects of Coulomb $\left(U_{\boldsymbol{Q}}\right)$ and screened electron-electron exchange $\left(V_{\boldsymbol{Q}}\right)$ interaction $[58,72]$, yielding

$$
\frac{4 g_{\boldsymbol{Q}}^{2}}{\hbar \omega_{\boldsymbol{Q}}}-2 U_{\boldsymbol{Q}}+V_{\boldsymbol{Q}} \geq \frac{1}{\chi(\boldsymbol{Q})}
$$

with the necessary hierarchy for CDW formation

$$
\frac{4 g_{\boldsymbol{Q}}^{2}}{\hbar \omega_{\boldsymbol{Q}}}>2 U_{\boldsymbol{Q}}>V_{\boldsymbol{Q}}>0 .
$$

In the absence of electron-phonon coupling $\left(g_{\boldsymbol{Q}}=0\right)$, this extended criterion shows that the Coulomb interaction $U_{\boldsymbol{Q}}$ outweighs the electron-electron exchange interaction $\left(V_{\boldsymbol{Q}}\right)$ and hinders the formation of a CDW. A beneficial situation, however, is realized for strong electron-phonon coupling $g_{\boldsymbol{Q}}$, large non-interacting susceptibility $\chi(Q)$, weak Coulomb interaction $U_{\boldsymbol{Q}}$ and small phonon energy $\hbar \omega_{\boldsymbol{Q}}$ [72].

The material studied in this thesis, $1 T-\mathrm{TaS}_{2}$, is a good example of a system that adheres to the principles outlined in the above discussion. The roles of the Fermi nesting mechanism and the strength of electron-phonon coupling $g_{\boldsymbol{Q}}$ are intimately connected to the emergence of various CDW phases exhibiting incommensurate as well as commensurate periodicities. In the low-temperature regime, electron-electron interactions $\left(U_{\boldsymbol{Q}}\right.$ and $\left.V_{\boldsymbol{Q}}\right)$ have an increasing influence and are believed to give rise to an insulating Mott state. The exact nature of this state is still under debate as it is challenging to access experimentally. Time-resolved techniques, in particular, could provide further information and elucidate important aspects [76].

\subsubsection{Fluctuation Effects}

The previously discussed mean field treatment neglects fluctuations in the system which results in a finite phase transition temperature for long range order. This, however, is an artefact of the 
chosen approach and does not hold for strictly one-dimensional systems with fluctuations. As mentioned in the introduction of this chapter, this leads to the nomenclature of quasi-one and quasitwo-dimensional systems. In this section, we discuss the role of fluctuations in a CDW system.

\subsubsection{Collective Excitations}

Among the various theoretical approaches, a common theoretical description of broken-symmetry states and their excitations is the phenomenological Ginzburg-Landau theory that has been widely and successfully used in describing structurally incommensurate phases [68, 77, 78]. Here, the breaking of a symmetry is described by a coordinate-dependent order parameter $\Delta(\boldsymbol{x}, t)$ that has a finite non-zero value in the low-symmetry phase while vanishing in the high-symmetry-phase [79-81]. This abstract object can be related to physically observable quantities, such as spontaneous magnetization or crystal lattice distortion, like in the present case for CDWs [82]. The system's equilibrium state is then determined by the global minimum of the order-parameter-dependent free-energy $F(\Delta(x, t))[83]$.

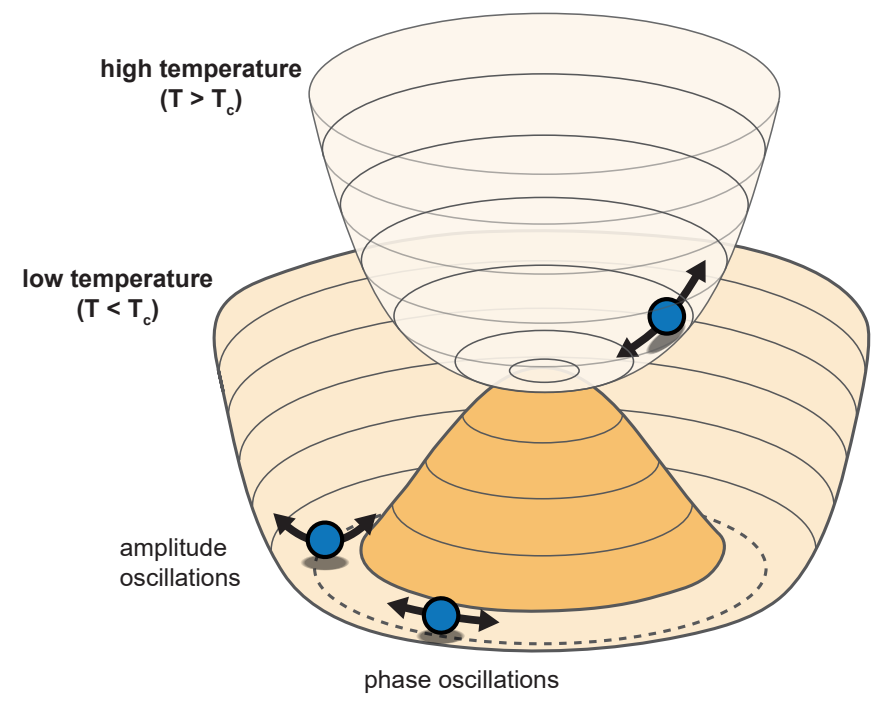

Figure 1.6: Symmetry-breaking phase transition described by a free-energy potential. For a transition described by a complex order parameter, the rotational symmetry yields a potential in the form of a champagne bottle bottom.

For the charge-density wave of a one-dimensional chain, the free-energy expansion is given by [26]

$$
F=F_{0}+n\left(\epsilon_{F}\right) \int d x\left[a|\Delta|^{2}+b|\Delta|^{4}+c\left|\frac{d \Delta}{d x}\right|^{2}+d\left|\frac{d \Delta}{d t}\right|^{2}\right],
$$

where $n\left(\epsilon_{F}\right)$ is the electron density at the Fermi energy, and $a, b, c$ and $d$ are known as the 
temperature-dependent Ginzburg-Landau parameters that can be derived from microscopic theories, like the Peierls-Fröhlich model introduced above.

For a complex order parameter, the form of the free-energy functional below and above $T_{c}$ is shown in Fig. 1.6. In the high-symmetry phase, the amplitude of the order parameter fluctuates around $|\Delta|=0$, whereas below $T_{c}$, the system's symmetry is spontaneously broken with fluctuations around a finite value of $|\Delta|$, in a potential whose shape resembles the bottom of a champagne bottle. The phase of the order parameter is continuous and can freely rotate in the minimum of the potential well [84].

in Equation 1.28, the third and fourth term refer to spatial and temporal changes of the fluctuating order parameter that can be assumed to have the form

$$
\Delta(\boldsymbol{x}, t)=[|\Delta|+\delta(\boldsymbol{x}, t)] e^{i\left(\varphi_{0}+\varphi(\boldsymbol{x}, t)\right)} .
$$

The quantities $|\Delta|$ and $\varphi_{0}(=0)$ are the spatially and temporally averaged amplitude and phase, whereas $\delta(\boldsymbol{x}, t)$ and $\varphi(\boldsymbol{x}, t)$ describe the fluctuations around the mean values. In this low-order approximation, the long-wavelength excitations of the condensate consist of two independent modes, the amplitude mode and the phase mode [26].

The concept of the spontaneous breakdown of a continuous symmetry has broad applicability in many fields of physics $[64,85-88]$. In quantum field theory, the amplitude and the phase excitations are also referred to as the Higgs [89] and the massless Nambu-Goldstone mode [90], respectively, named after its discoverers. For a rigorous mathematical derivation of symmetry breaking from a field-theoretical point of view, we refer to Ref. [91] published by Strocchi. In his lecture notes, he unfolds in detail the depths and subtleties of this mechanism, avoiding the 'standard folklore explanations'.

The altered phonon dispersion of CDW-coupled modes in the vicinity of $\boldsymbol{Q}=2 \boldsymbol{k}_{F}+\boldsymbol{\delta} \boldsymbol{q}$ has been calculated by Lee, Rice, and Anderson [92] within a perturbative approach, i.e., small fluctuations, based on the microscopic Fröhlich Hamiltonian, and yields for zero temperature

$$
\begin{aligned}
\omega_{\mathrm{pha}}^{2}(\boldsymbol{\delta} \boldsymbol{q}) & =\frac{m}{m^{*}} v_{F}^{2} \boldsymbol{\delta} \boldsymbol{q}^{2} \equiv v_{\varphi}^{2} \boldsymbol{\delta} \boldsymbol{q}^{2} \\
\omega_{\mathrm{amp}}^{2}(\boldsymbol{\delta} \boldsymbol{q}) & =\lambda \omega_{2 k_{F}}^{2}+\frac{4}{3} \frac{m}{m^{*}} v_{F}^{2} \boldsymbol{\delta} \boldsymbol{q}^{2},
\end{aligned}
$$

where $v_{\varphi}$ denotes the phason sound velocity, and the ratio of the effective mass $m^{*}$ and the band mass $m$ [92] is given by

$$
\frac{m^{*}}{m}=1+\frac{4 \Delta^{2}}{\lambda \omega_{2 k_{F}}^{2}}
$$


with the dimensionless electron-phonon coupling constant

$$
\lambda=\frac{p g^{2}}{\hbar \omega_{2 k_{F}} \epsilon_{F}}
$$

and the number of conduction electrons per atom $p$. The ratio of the effective mass ratio $m^{*} / m$ enters the dispersion expressions since the CDW-coupled lattice modes respond slower than that of electronic system [92]. A similar expression for the effective mass $m^{*}$ was derived by Fröhlich [33].
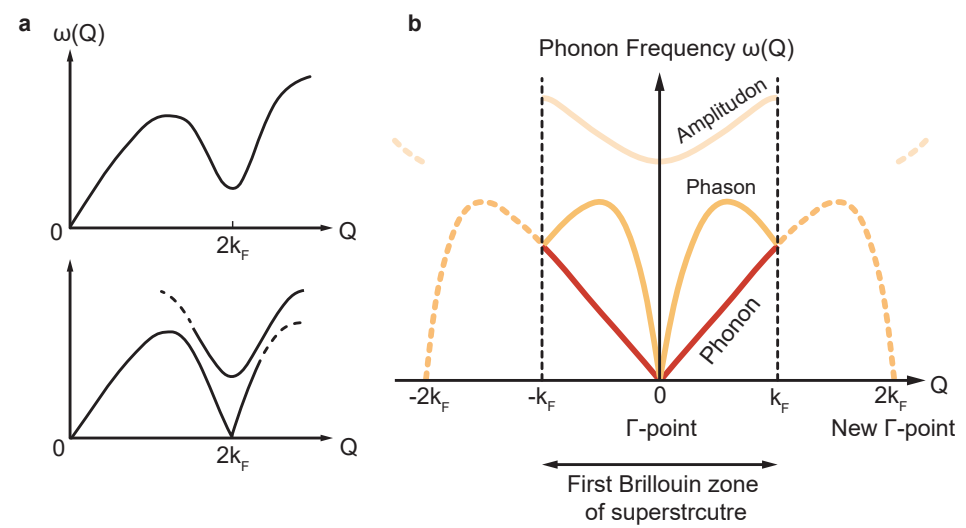

c

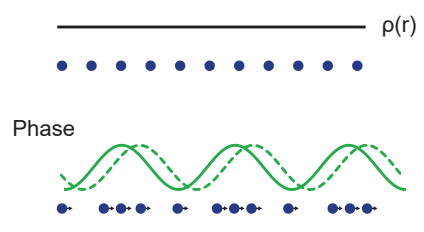

Amplitude

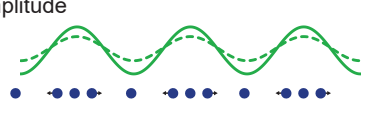

Figure 1.7: Schematic illustration of the dispersion relation for a material undergoing an incommensurate displacive phase transformation. Adapted from [93] (a) A phonon branch softens above $T_{c}$ at $\boldsymbol{Q}=2 \boldsymbol{k}_{F}$, and splits up into a gapless phason branch and an upper amplitudon branch below $T_{c}$. (b) Sketch of backfolded dispersion of CDW modes. Adapted from Ref. [55, 94, 95]. (c) Illustration of (sliding) phason and amplitude modes in a $1 \mathrm{~d}$ atomic chain.

a

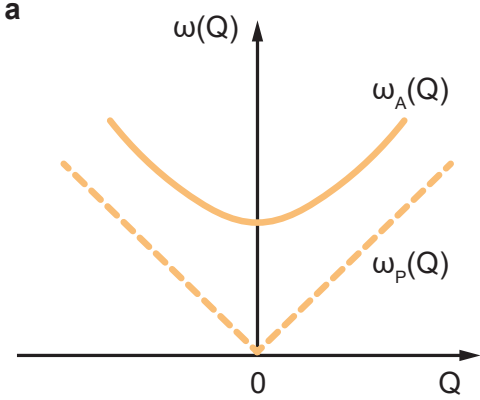

b

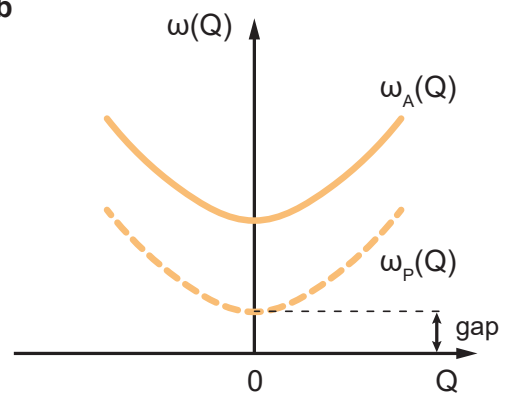

Figure 1.8: Sketch of the dispersion relation for an incommensurate structure featuring phase (dashed line) and amplitude (solid line) fluctuation modes. (a) A free-floating phason exhibits linear dispersion whereas (b) pinning of the phase by commensuration lock-in terms or impurities result in a gap of the phase excitation spectrum. Adapted from Ref. [96].

As shown in Figure 1.7, CDW-coupled modes emerge as a result of a soft lattice mode (see Fig. 1.7a) and introduce a new $2 \boldsymbol{k}_{F}$-periodicity leading to an altered Brillouin zone with backfolded branches 
of these new vibrational modes [84]. In terms of the order parameter introduced in Equation 1.15, the upper branch (see Fig. 1.7b) corresponds to a spatio-temporal modulation of the amplitude of the CDW, more specifically, the totally symmetric soft mode [68] or amplitudon. The lower branch, on the other hand, corresponds to the phase fluctuations of the order parameter, also called phasons $[92,94]$. The phason is expected to be optically active because of a non-vanishing dipole moment [92], whereas the amplitude mode is a Raman-active mode [26].

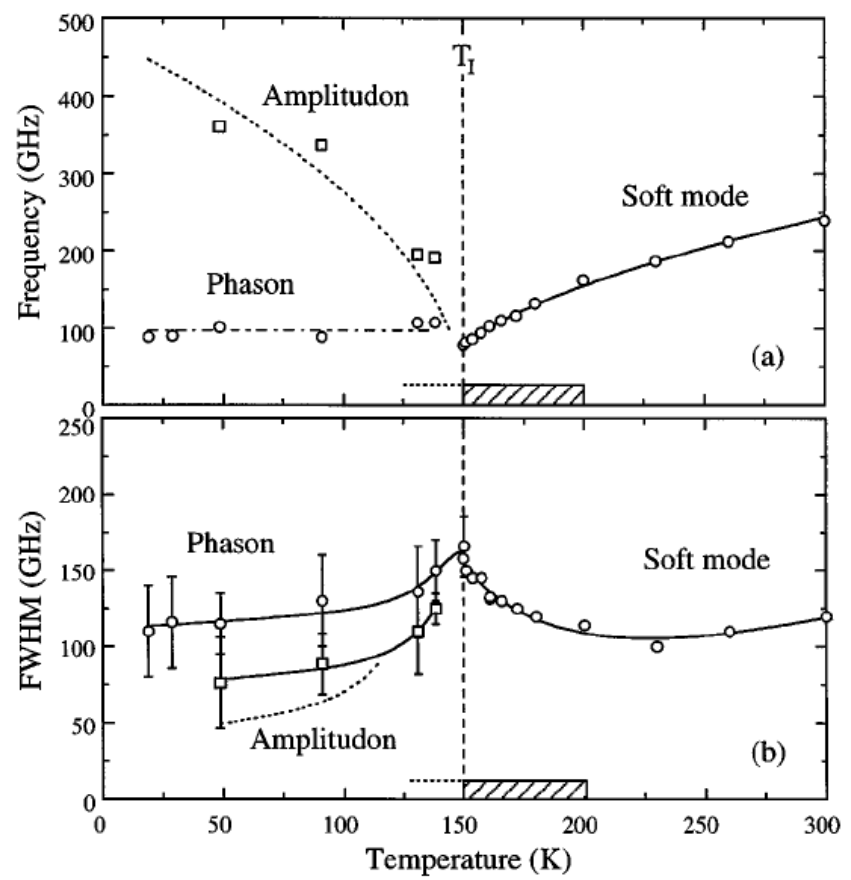

Figure 1.9: Frequencies (a) and damping (b) of the soft phonon $\left(T>T_{c}=150 \mathrm{~K}\right)$, phase and amplitude mode $\left(T<T_{c}\right)$ in BCPS obtained from neutron scattering measurements [84]. This insulating molecular compound undergoes a second order structural, displacive IC phase transition at $T_{c}$. One main characteristic is the underdamped soft mode in the high temperature phase that splits up into a amplitude and phase mode below $T_{c}$. From Refs. [84, 97]

While amplitudons as an optical mode always exhibit a finite energy gap, in an incommensurate structure, phasons are soft modes with vanishing excitation energy at $\boldsymbol{\delta} \boldsymbol{q}=0$. The low-energy excited phasons around this point can be thought of as a homogenous change of the phase or a sliding motion of the CDW which corresponds to the already introduced sliding mode (see Fig. 1.7c). For this reason, such modes were expected to carry current in a resistance-free manner [33, 92]. However, the sliding motion of the CDW is usually hindered by commensurate locking to the atomic lattice or pinning due to inhomogeneities $[71,96]$ removing the translational invariance in their presence. Consequently, the forming CDW does not break a continuous symmetry and 
completely softened modes should not emergence. Instead, phason modes exhibit an electronic gap with a finite excitation energy (see Fig. 1.8) [54].

As a result, for example shown by conductivity and neutron scattering measurements on quasione-dimensional systems $[71,74,98]$, the oscillator strength of the CDW sliding mode is shifted from zero to finite frequency $[84,99]$ and is referred to as the pinned phase mode [96]. It is interpreted as a 'bound' collective mode arising from the presence of polarisable impurities [71]. Nonlinear transport properties were reported by Sinchenko [100] for layered $\mathrm{DyTe}_{3}$, demonstrating the sliding in a quasi-2d compound with striped CDW order [71, 101] while in materials systems with $2 \mathrm{~d} \mathrm{CDW}$ order, $2 \mathrm{H}-\mathrm{TaSe}_{2}$ and $1 T-\mathrm{TaS}_{2}$, such a current-voltage nonlinearity was not observed, possibly due to efficient commensurability or impurity pinning [102].

a

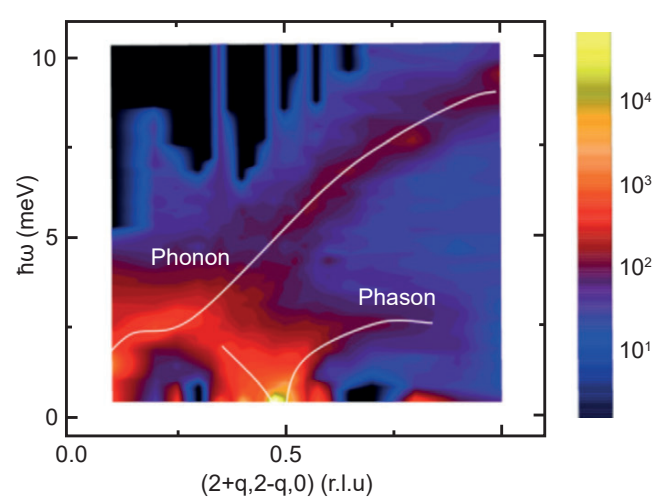

b

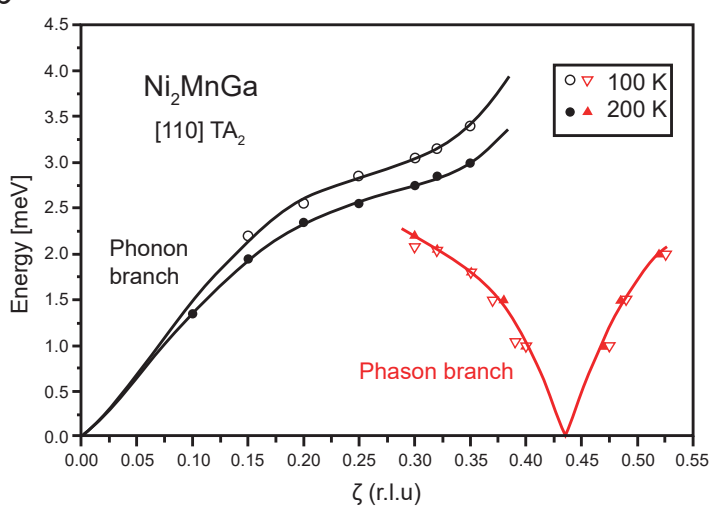

Figure 1.10: Neutron scattering experiment to retrieve phonon dispersion of the CDW material $\mathrm{Ni}_{2} \mathrm{MnGa}_{\text {. }}$ (a) Intensity contours determined from a series of constant $\boldsymbol{Q}$ scans measured with PUMA at FRM-II reactor. Black areas were outside the scan limits. The lines are guides through the intensity maxima. (b) Low-energy phonon dispersions measured along the transverse [110] direction. Measurements were made with FLEX at BENSC at Hahn Meitner Institute reactor. Adapted from Ref. [103] by Shapiro et al., licensed under CC BY 3.0.

Moreover, in many incommensurate structures, including non-CDW materials, it has been shown by means of inelastic neutron scattering that phason excitations exhibit a finite life-time due to dissipative coupling [80]. In the notation of various pinning models, a damping factor $\left(\Gamma_{\varphi}\right)$ is introduced that is defined through a phenomenological CDW viscosity [96]. Depending on the magnitude of this damping factor, the phason mode is diffusive instead of propagating [70, 104, 105]. The damping of phase excitations strongly depends on the material system and has been shown to be overdamped for, e.g., potassium selenate $\left(\mathrm{K}_{2} \mathrm{SeO}_{4}\right)$ [106] and betaine calcium chloride dihydrate [107], but underdamped for thorium tetrabromide $\left(\mathrm{ThBr}_{4}\right)$ [106], biphenyl [106, 108] and BCPS [84] (see Fig. 1.9). From experimental observations, values of the gap size and the damping 
constant of the collective excitation modes were found to be in a similar order of magnitude of $0.1 \mathrm{meV}[84,109,110]$.

CDW excitations have been well investigated in the past with many steady-state techniques [68, 110-114]. Since the damping of phasons and amplitudons is of similar strength and mostly $\boldsymbol{Q}$ independent, phasons in the vicinity of $\boldsymbol{Q}=2 \boldsymbol{k}_{F}$ are inevitably strongly damped and therefore very challenging to study. Among the experimental approaches, neutron scattering stood out as being well suited to map large parts of the material's vibrational spectrum providing first unambiguous evidence for phase excitations in incommensurate structures [68]. Exemplary, Figure 1.10 shows the vibrational spectrum of the $3 \mathrm{~d}$ metallic $\mathrm{CDW}$ compound $\mathrm{Ni}_{2} \mathrm{MnGa}$ featuring normal phonon modes and a well-defined phason branch [103].

\subsubsection{Domains}

Small CDW fluctuations led to the emergence of collective excitations, namely amplitudons or phasons, whereas large spatio-temporal fluctuations of the order parameter give rise to additional types of excitations, such as domain structures or CDW dislocation defects [78, 115]. Here, we briefly sketch two scenarios in which these special objects naturally arise [54].

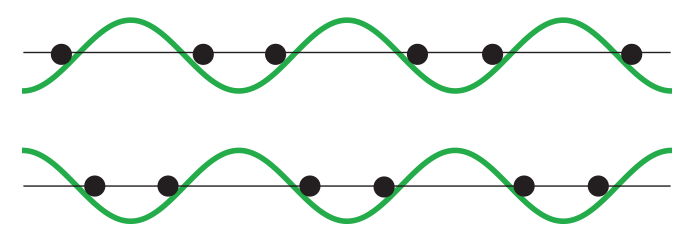

Figure 1.11: The dimerized lattice (dots) exhibits two energetically equivalent deformation configurations in the CDW state (green lines). Adapted from Ref. [54].

First, we consider once more a half-filled $1 \mathrm{~d}$ chain of atoms that commensurably dimerizes due to a $2 \boldsymbol{k}_{F}$ instability discussed above. The CDW phase is commensurate to the lattice resulting in a two-fold $(\varphi=0$ or $\varphi=\pi)$ degenerate ground state configuration for the lattice deformation (see Fig. 1.11). In the more general case of a $m$-fold commensurability, the ground state exhibits $m$ energetically equal configurations determined by the phase-dependent commensurability energy

$$
E_{\mathrm{comm}} \sim 1-\cos (m \varphi)
$$

Large phase fluctuations between the two ground states, defined by non-zero integer multiples of $2 \pi / m$, lead to different lattice deformations in these intervals. At the meeting points, a characteristic border will form compensating for the phase difference by either straining or compressing the lattice 
(see Fig. 1.12). Determined by the outer phase boundary condition of the system, the domain wall is topologically protected and cannot decay, leading to emerging domains of different phase. In theory, this is reflected by a sine-Gordon-type equation that incorporates the commensurability energy (equation 1.34) and yields solitary wave solutions that are interpreted as edges with an invariant shape [54].

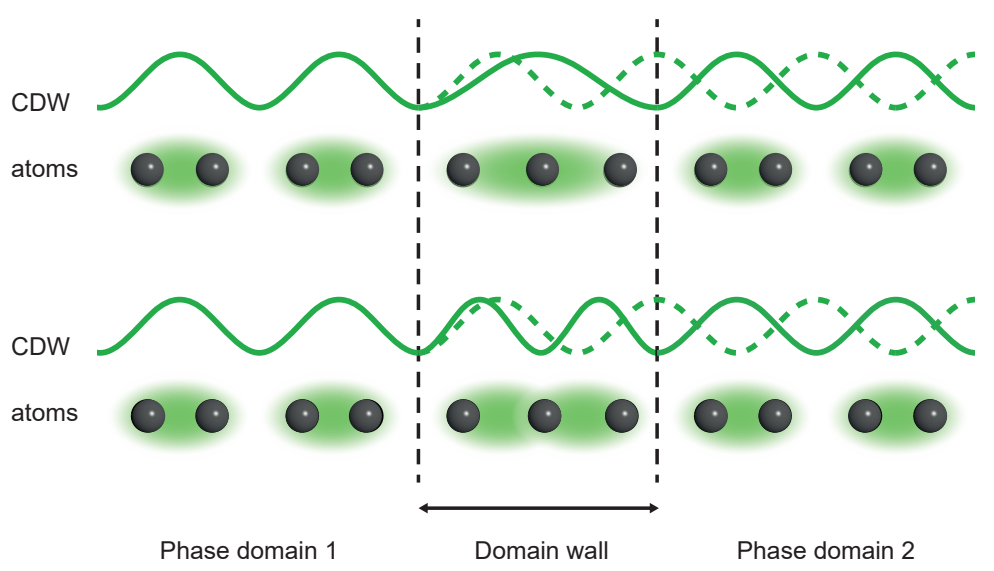

Figure 1.12: Phase compartmentalization. Where two oppositely dimerized domains meet, the density wave is either stretched (top) or compressed (bottom), creating a domain wall with different periodicity. Adapted from Refs. [54] and [56].

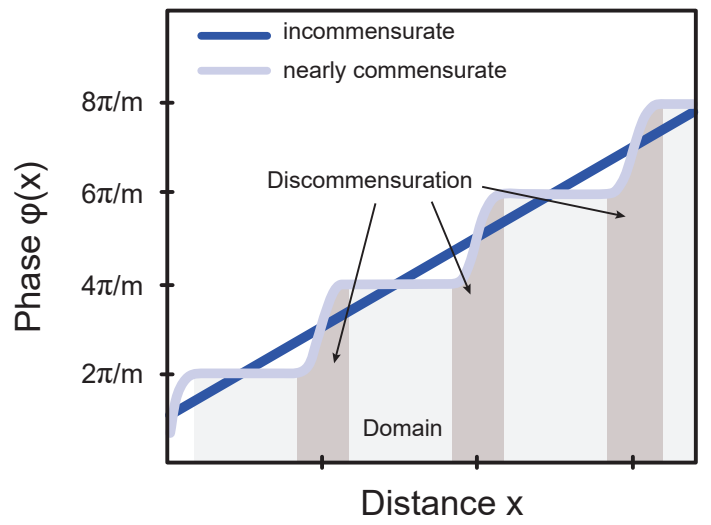

Figure 1.13: Phase jumps in the close to commensurate case. The CDW phase locally registers with the atomic lattice creating commensurate patches that are separated by discommensurations. Adapted from Ref. [116].

A particularly interesting case occurs when the lattice experiences a distortion by an incommensurate $\mathrm{CDW}$, whose wavelength $\lambda_{\mathrm{CDW}}$ is close to a multiple of the atomic lattice constant $a$. For the lattice, it may now be energetically favorable to locally increase commensurability within finite regions by 
pinning the phase to the atomic lattice. As a result, the emerging domains of constant (or slowly changing) phase are separated by domain walls that correspond to a fast spatial variation of the phase, also known as discommensurations $[78,116]$ (see Fig. 1.13). This phenomenon is not unique to CDW systems and was also studied theoretically very early in connection with adsorbates on substrates by Frenkel, Kontorowa and van Merwe, amongst others [116-122]. The local increase in commensurability also affects the spectrum of collective excitations and introduces a finite-energy phason (see Fig. 1.8).

As a matter of fact, the described scenario of patch formation is realized in the nearly commensurate $\mathrm{CDW}$ phase of $1 T-\mathrm{TaS}_{2}$ and can be experimentally observed at room temperature (see Sec. 1.3).

\subsubsection{CDW Dislocation Defects}

A genuinely incommensurate CDW phase interacts very weakly with the underlying atomic lattice and is often considered to be free-floating [80]. Any phase jumps of the type shown in Fig. 1.12 that are present in the system, for example, externally introduced by intense laser irradiation, will decay over time, restoring the slowly-varying phase dependency. Coming to the second scenario, this statement does not necessarily hold going to two-dimensional incommensurate CDW systems that usually exhibit a specific kind of crystalline order.

More specifically, McMillan [77] constructed the hexagonal CDW lattice of transition-metal dichalcogenides by superimposing complex order parameters in the form of three phase-locked complex plane waves

$$
\psi_{j}(\boldsymbol{r})=\phi_{j} \exp \left(i \boldsymbol{Q}_{j} \boldsymbol{r}\right), \text { for } j=1,2,3,
$$

where $\phi_{j}$ are complex amplitudes and $\boldsymbol{Q}_{j}$ the wave vectors, rotated by an angle of $120^{\circ}$ relative to each other. Based on a Landau free-energy of the incommensurate phase, he showed that dislocationtype defects may emerge in such CDW lattices, much like vortices in superfluidic helium and superconductors [77]. In the CDW, a single defect (see Fig. 1.14a and b; note two CDW dislocation defects are shown) consists in this picture of two pitchfork dislocations in two of the three complex order parameters where the amplitude vanishes in the center of the dislocation (Fig. 1.14c) and the phase adds to $\pm 2 \pi$ on a closed contour around the phase singularity (Fig. 1.14d). In the CDW lattice, this translates to two additionally inserted rows (Fig. 1.14b). CDW dislocation defects, each characterized by a Burgers vector $\boldsymbol{b}$, emerge as pairs that can move, annihilate or recombine to a different type.

Generally, an incommensurate phase in two dimensions presents a particular interesting case for the study of correlation effects [80]. As mentioned above, the non-existence of long-range order in 
a

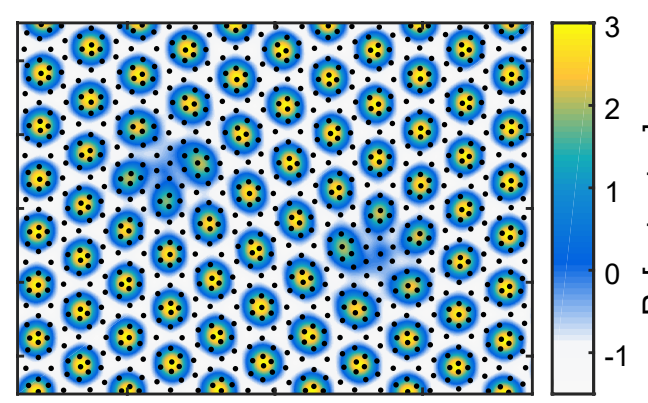

C

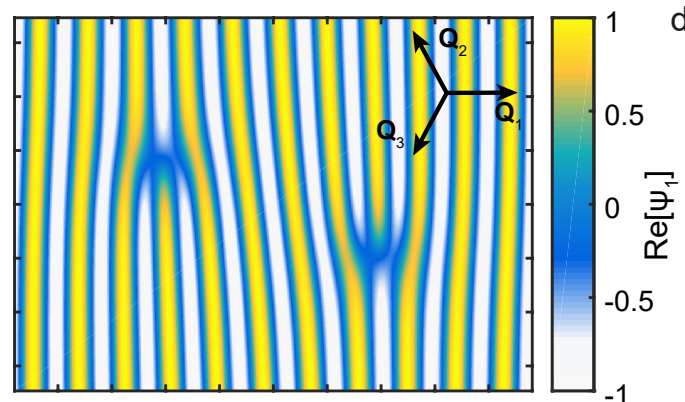

b
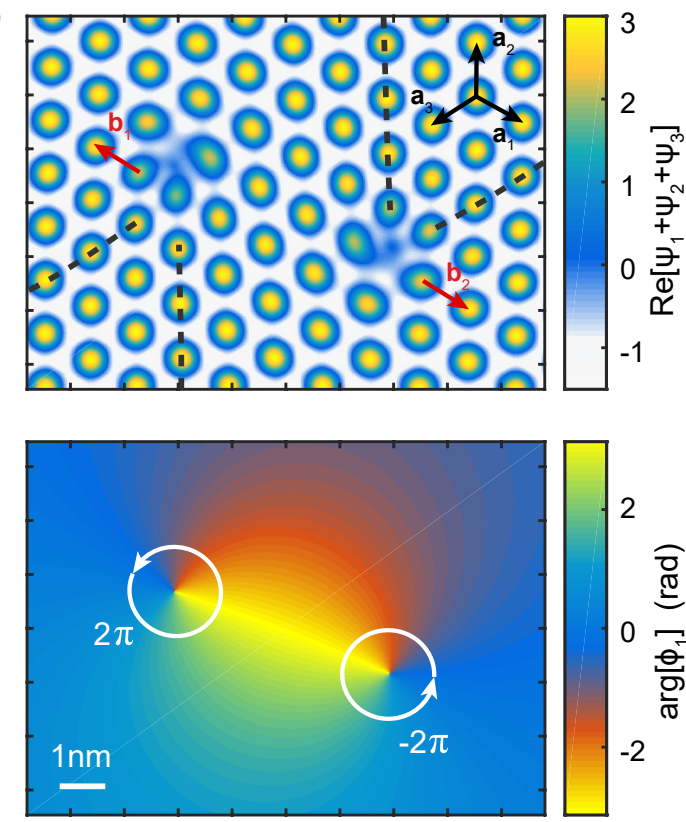

Figure 1.14: Dislocation-type topological defects. (a) Real part of the summed complex order parameters showing a hexagonal CDW dot-lattice (blue and yellow) and a CDW dislocation defect pair. Atoms are shown as black dots. (b) The individual defects are characterized by a Burgers vector (red arrows) or two additionally inserted CDW lattice rows (dashed lines). (c) The real of part of a single contribution to the triple CDW $\left(\psi_{1}\right)$ exhibits two pitchfork dislocations, one for each individual CDW dislocation defect. (d) The phase of $\psi_{1}$ shows two singularities that can be characterized by the value of the line integral around the defect site, yielding $\pm 2 \pi$. From Ref. [56].

two dimensions also prevents the formation of an incommensurate order over a long distance. The correlation function of the 'floating' I phase exhibits therefore an additional term that introduces an algebraic decay which is believed to be unique to two-dimensional systems [116]. Additionally, the system can undergo a phase transition into a so-called fluid phase that is connected to the unbinding of dislocations or vortices. The theory of such a microscopic melting process in two dimensions was first put forward by Kosterlitz, Thouless, Halperin and Young [80]. The correlation functions of the order parameter in the various phases take the following forms at long distances [116]:

$$
\begin{aligned}
\text { incommensurate: }\langle\Delta(0) \Delta(\boldsymbol{r})\rangle \sim \cos (\boldsymbol{Q} \cdot \boldsymbol{r}+\varphi) \\
\text { floating I: }\langle\Delta(0) \Delta(\boldsymbol{r})\rangle \sim r^{-\eta} \cos (\boldsymbol{Q} \cdot \boldsymbol{r}+\varphi) \\
\text { fluid: }\langle\Delta(0) \Delta(\boldsymbol{r})\rangle \sim e^{-\kappa r} \cos (\boldsymbol{Q} \cdot \boldsymbol{r}) \\
\text { commensurate: }\langle\Delta(0) \Delta(\boldsymbol{r})\rangle \sim \cos \left(\boldsymbol{Q}_{0} \cdot \boldsymbol{r}+\varphi\right), \boldsymbol{Q}_{0} \text { commensurate, locked, }
\end{aligned}
$$

where $\eta$ and $\kappa$ are constants. In a recent work, we showed that the phase-ordering kinetics of the 
laser-induced NC-IC phase transition in $1 T-\mathrm{TaS}_{2}$ is governed by the creation and annihilation of CDW dislocation defects [14].

\subsection{Aspects of Low-energy electron diffraction}

The chemical and physical properties of matter are strongly influenced by its specific structural order. It is therefore highly desirable to determine the precise relative positions of an ensemble of atoms. This goal can be achieved with different types of probes, often constituted of directed particle beams (see Fig. 1.15).

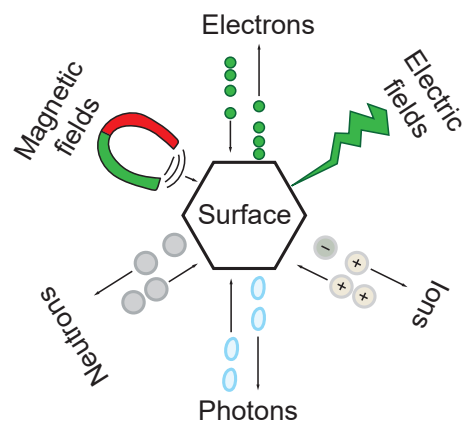

Figure 1.15: Sketch of different types of probes to investigate surfaces. Adapted from Ref. [123].

A particularly successful way of structure determination of periodic crystalline surfaces makes use of diffracting low-energy electrons. This technique is known as low-energy electron diffraction (LEED). Generally, diffraction is a very powerful tool when it comes to ordered structures because suitable interference conditions allow for clear diffraction reflexes and an unaltered insight into the structure. Moreover, small transient deviations from perfect order heavily influence the interference condition and strongly alter the observable signals.

Furthermore, low-energy electrons interact strongly with matter, hence, scatter efficiently and do not penetrate far into the sample in comparison to other probes like x-ray radiation or high-energy electrons. While a large portion of backscattered electrons lose energy through various inelastic scattering channels (see Fig. 1.16a), a few percent are elastically diffracted and contain information about the average crystal atom positions. Inelastically scattered electrons are recorded as diffuse background and offer a rich insight to excited lattice modes. The exact penetration depth, mainly determined by the inelastic mean free path, weakly depends on the specific material investigated and follows a universal, nearly constant trend in the range of tens up to few hundreds electron volts (see Fig. 1.16b). In this range, electrons penetrate several Ångström $\left(\AA=10^{-10} \mathrm{~m}\right)$ into the sample, which corresponds to a probing volume of few atomic layers. In order to map a typical crystalline 
a

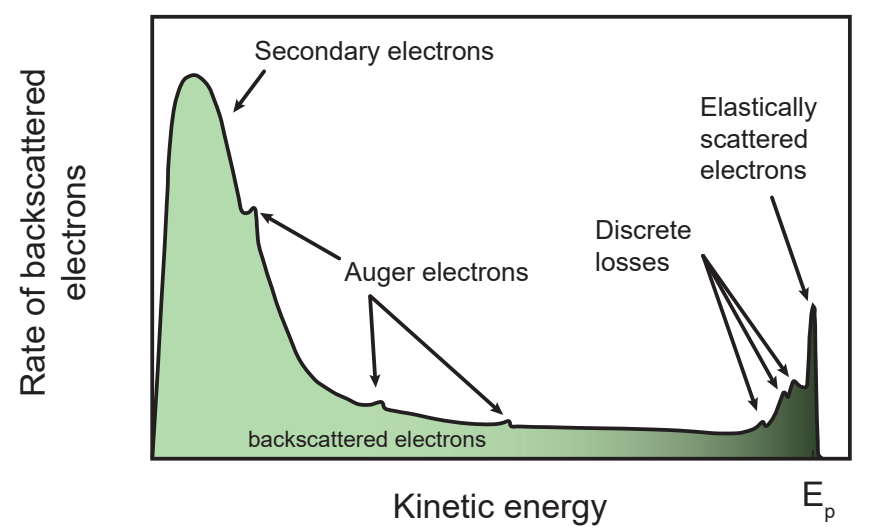

b

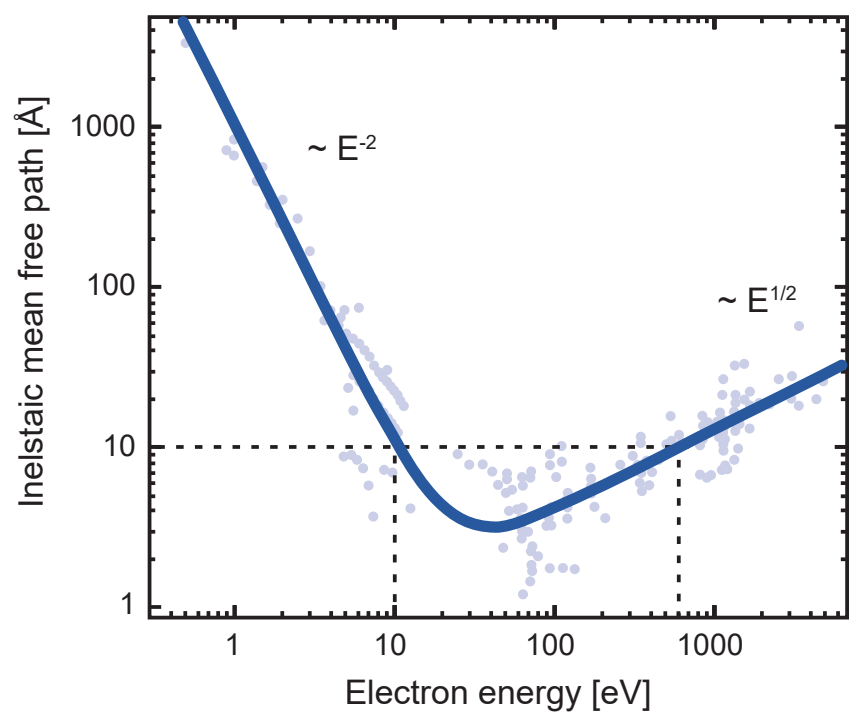

Figure 1.16: Electron scattering. (a) Schematic illustration of the energy distribution of backscattered electrons. A large fraction of the electrons loses energy by various scattering processes. A few percent are scattered elastically at an energy of $E_{p}$ (primary electrons). Adapted from [123, 124]. (b) Inelastic mean free path as a function of the kinetic electron energy. The curve (solid) shows the universal trend through the individual measurements on different surface elements (light blue dots). Adapted from Refs. [123, 125, 126].

structure using a diffraction technique, the wavelength of the incident beam should be smaller or equal to the lattice constant [127]. For low-energy electrons having an energy $E$, the de Broglie wavelength is given by

$$
\lambda=\sqrt{\frac{150.4}{E[\mathrm{eV}]}} \AA,
$$

where an electron volt is defined by $1 \mathrm{eV}=1.602 \cdot 10^{-19} \mathrm{~J}$. The wavelength of typical energies ranging from 30 up to $500 \mathrm{eV}$ is comparable to common lattice spacings of solids. The low penetration depth, the high scattering efficiency and the suitable wavelength range turn LEED into 
an ideal probe to investigate the structure of surfaces [14], thin films [46] or adsorbed molecules [50].

\subsubsection{Experimental LEED Setup}

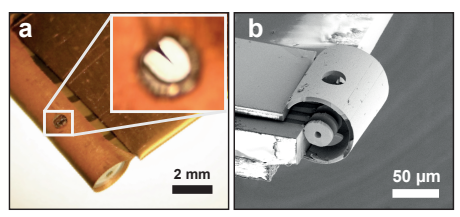

c UHV chamber

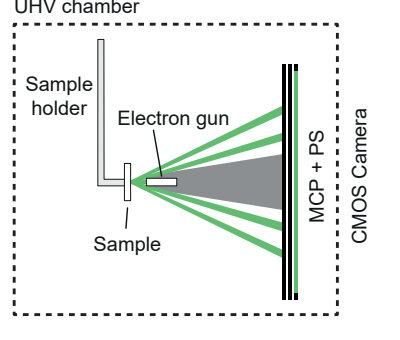

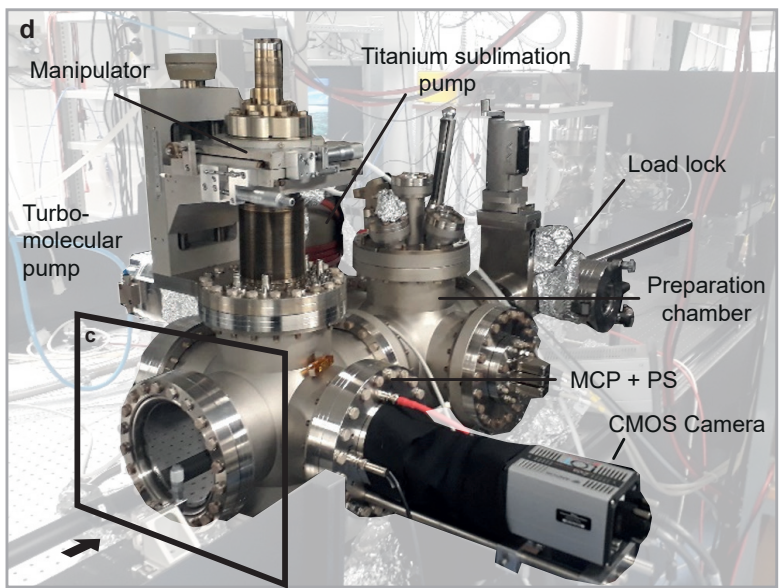

Figure 1.17: Experimental setup of LEED. (a) Millimeter-sized laser-driven electron gun. Inset: tungsten tip, view through the hole for laser illumination. Reprinted from Ref. [14]. (b) Micrometer-sized electron gun, prepared using nanofabrication techniques. From [14]. (c) Schematic of low-energy electron scattering in backreflection (see also Fig. D.1). (d) Photograph of ULEED measurement chamber featuring essential parts of a LEED setup.

In this section, the main experimental components of our LEED setup are briefly described. For surface diffraction experiments, it is important to prepare clean samples and avoid that impurities are adsorbed on the surface. A preparation process and measurement inside ultra-high vacuum (UHV) conditions (pressure $10^{-10}-10^{-11} \mathrm{mbar}$ ) is therefore advantageous since the amount of remaining residual gas in the vacuum chamber is strongly reduced. In order to obtain a stable UHV environment, an array of different types of vacuum pumps (in our case, a rotary vane pump, a turbomolecular pump and a titanium sublimation pump, see Fig. 1.17d) is installed, and a baking procedure of the chamber is carried out.

For the measurements, the sample is mounted on a manipulator that allows for sample translation and rotation in multiple axis. An optimized crystal orientation during the experiment can remove asymmetries in the diffraction pattern and simplify the analysis of the measured data.

Diffracted electrons are typically detected by a fluorescent screen for position-sensitive measurements (see Fig. 1.17c). The detector is recorded with a suitable camera system that provides experimental data in the form of diffraction images (see also Sec. E.1). The backscattering angles 
of the diffracted beams depend on the lattice constant of the sample and the specific electron energy, but typically lie in the range of several degrees, which is much larger compared to high-energy electron diffraction experiments. For low beam currents, as in our experiment, it is necessary to enhance the diffracted electron signal with a multi-channel plate (MCP) before detecting it on the fluorescent screen. Most commonly, the diffracted signal passes a retarding field energy analyzer capable of filtering scattered electrons or removing a constant diffuse background.

Generally, the achievable quality of scattering experiments depends strongly on the performance of the electron source. Commercial LEED guns are usually based on heated (tungsten) filaments providing beam currents of $10^{-8}-10^{-4} \mathrm{~A}$ in an energy range of $30-1000 \mathrm{eV}$ and electron optics allowing for electron foci of approximately $1 \mathrm{~mm}$ [125]. A further technical advantage of LEED for the manipulation of electron trajectories is the rather simple handling of low electric voltages compared to diffraction techniques at electron energies of several tens of $\mathrm{keV}$.

In the context of this thesis, the development of new electron gun designs (see Fig. 1.17a,b and D.1) was pursued for the purpose of a time-resolved realization of LEED, namely ULEED. For the details of the fabrication process of the micro-meter sized gun, we refer to Chapter 2 (or Ref. [47]), and for the concept of ULEED to Chapter 3 (or Refs. [14, 46, 56]). The first time-resolved results using the micro-meter sized gun are published in Ref. [14] by Vogelgesang et al. on the NC-IC transition of $1 T-\mathrm{TaS}_{2}$.

\subsubsection{Instrumental Response Function}

In this section, we shortly discuss the effect of the instrumentation on the quality of the diffraction pattern. This will lead to the so-called transfer width, which is a measure for the performance of a source used in a diffraction setup.

In an ideal scenario, diffraction with a perfect instrument on a flawlessly periodic, rigid and infinite crystal would result in infinitely sharp observed LEED reflexes. However in real experimental conditions, diffraction peaks exhibit a finite width due to instrumental distortions and surface imperfections. Some details of the impact of imperfections are discussed in the chapter on scattering theory 1.2.3.

In order to quantify the effect of the experimental setup, the so-called instrumental response function $T(s)$ is introduced. This function captures the broadening of the ideal intensity distribution $I(s)$ that would be measured with a perfect instrument. The major contributions to the broadening are a finite energy width of the incident electron beam, the electron beam diameter, the (virtual) electron source size and the aperture width of the detector [125]. Mathematically, the observed 
intensity $J(s)$ is expressed via the convolution

$$
J(s)=T(s) * I(s)=\int T(s) \cdot I\left(s-s^{\prime}\right) d s^{\prime},
$$

where $s$ is the scattering vector quantifying the momentum transfer. The Fourier transform of the observed quantity $J(s)$ is given by

$$
\mathcal{F}\{J(s)\}=\mathcal{F}\{T(s) * I(s)\}=\mathcal{F}\{T(s)\} \cdot \mathcal{F}\{I(s)\}=t(\boldsymbol{r}) \Phi(\boldsymbol{r}),
$$

where $t(\boldsymbol{r})$ denotes the transfer function and $\Phi(\boldsymbol{r})$ the autocorrelation or pair correlation function [123]. $\Phi(\boldsymbol{r})$ provides structural information and is a measure for the degree of ordering for a given vector $\boldsymbol{r}$. The following chapter shows that the diffraction intensity $I(s)$ can be obtained from the exact distribution of the atoms. It should be noted, however, that the reverse is not readily achievable due to the lack of the scattering phases. The autocorrelation function is modulated by the transfer function $t(\boldsymbol{r})$ whose full-width at half-maximum (FWHM) is called transfer width. The transfer width can be regarded as an effective coherence length of the entire instrumentation, setting an upper limit for the size of the observable spatial period in the sample. In particular, the correlation between two scatterers separated by $\boldsymbol{r}$ is undetectable if the value of $t(\boldsymbol{r})$ vanishes. Typical values of the transfer width for commercial LEED setups lie in the range of $20-120 \AA$ [125].

The electron guns developed during this thesis (see Fig. 1.17a,b) exceed these typical values and exhibit a transfer width of $200-250 \AA$ [14, 47]. The performance enhancement is due to the significant reduction of the virtual electron source size, high electron monochromaticity and a reduced electron beam diameter (between 10-100 $\mu \mathrm{m}$ ), achieved by using a nanometric photoemitter $[46,128]$ and a miniaturized gun design (see Chapter 2).

\subsubsection{Scattering Theory}

Generally, the scattering process of low-energy electrons inside a crystal consists of multiple consecutive scattering events due to the strong interaction with the atomic potentials. In a detailed theory, all of these scattering events must be accounted for in order to fully describe every feature of diffraction intensity profiles [125]. Such dynamical LEED calculations were employed in our group in order to fully resolve the surface structure of the C-phase of $1 T-\mathrm{TaS}_{2}$ [129].

For this introductory section, a simpler theory is used since multiple scattering generally has little qualitative effect on the LEED pattern and, for example, does not change the diffraction spot positions [125]. In this theory, it is assumed that the electron interaction is sufficiently weak such that multiple scattering can be neglected. As a result, the diffraction problem is reduced to a singlescattering theory, also known as a kinematic theory. For example, in x-ray and high-energy electron 
diffraction, this weak-interaction approach has real practical value in analyzing diffraction patterns and is successfully applied [125].

In general, electron beams behave as waves with a characteristic wavelength and wave vector. A simple example of scattering yielding structural information is illustrated in Figure 1.18. An incoming plane wave diffracts on a $1 \mathrm{~d}$ chain of equally spaced atoms and is modulated by constructive and destructive interferences due to the path difference $d$. The condition for constructive interference, also known as Bragg condition, is fulfilled if the difference in path length between two neighboring scatterers is a multiple of the wavelength

$$
n \lambda=a \sin (\theta)
$$

where $n$ is an integer number and $a$ the lattice constant. This elastic type of scattering gives rise to characteristic reflexes, the so-called Bragg peaks.

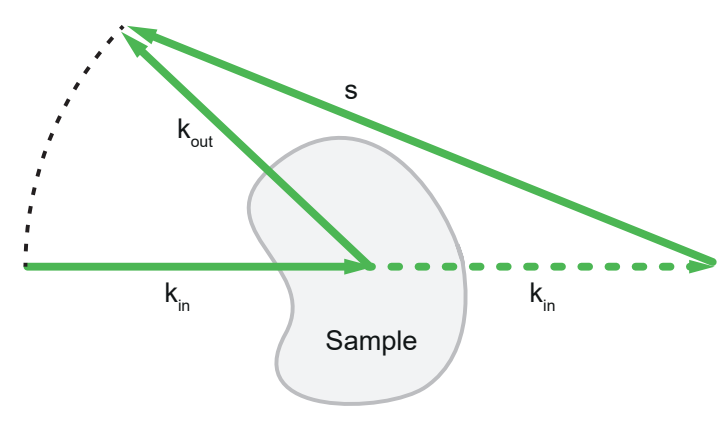

b

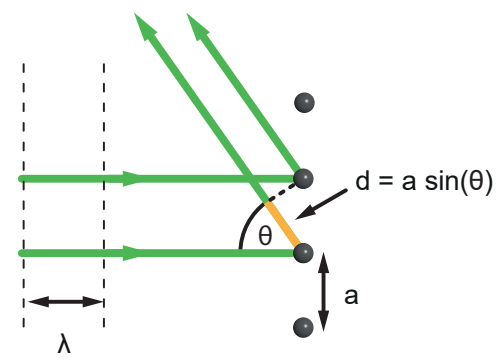

Figure 1.18: Sketch of a simple scattering event. (a) An incoming plane wave with a wavevector $k_{\text {in }}$ diffracts at a sample resulting in a backpropagating wave with wave vector $k_{\text {out }}$. The scattering vector $s$ is the difference of $\boldsymbol{k}_{\text {in }}$ and $\boldsymbol{k}_{\text {out }}$. (b) In Bragg scattering, constructive or destructive interference is controlled by the path difference $d$ between two neighboring scatterers. Adapted from Ref. [130].

Bragg scattering always emerges when an electron beam hits a inhomogeneous medium, in particular for periodic structures like crystals. A crystalline lattice is an infinite periodic array of indistinguishable sites in up to three dimensions. This Bravais lattice is spanned by a position vector $\boldsymbol{R}$, given by

$$
\boldsymbol{R}=n_{1} \boldsymbol{a}_{1}+n_{2} \boldsymbol{a}_{2}+n_{3} \boldsymbol{a}_{3}
$$

where $n_{1}, n_{2}$ and $n_{3}$ denote integer numbers and $\boldsymbol{a}_{1}, \boldsymbol{a}_{2}$ and $\boldsymbol{a}_{3}$ are linearly independent lattice vectors. In order to properly describe all types of crystals, a lattice site can be decorated by several atoms, called basis atoms. With the sum of lattice and basis vector $\boldsymbol{R}+\boldsymbol{t}_{i}$ all atoms inside a crystal can be reached. The vector $\boldsymbol{t}_{j}$ alone gives all atoms in a unit cell. The lattice can be considered to define volumes, the unit cells, which together fill the space and form the crystal. 
In a more elaborate approach, the scattering of an incoming plane electron wave from a single atom potential is described via the Schrödinger equation. In this picture, the amplitude of the outgoing spherical wave is determined via the Fourier transform of the electric potential [131] and is given by

$$
A(\boldsymbol{s})=\frac{1}{4 \pi} \int U\left(\boldsymbol{r}^{\prime}\right) e^{-i s \cdot r^{\prime}} d r^{\prime} .
$$

For a crystal, the total potential is the sum of an array of atomic potentials

$$
U(\boldsymbol{r})=\sum_{j} U_{j}^{a}\left(\boldsymbol{r}-\boldsymbol{r}_{j}\right)
$$

leading to a scattering amplitude

$$
A(\boldsymbol{s})=\frac{1}{4 \pi} \int U\left(\boldsymbol{r}^{\prime}\right) e^{-i \boldsymbol{s} \cdot \boldsymbol{r}^{\prime}} d r^{\prime}=\frac{1}{4 \pi} \sum_{j} e^{-i \boldsymbol{s} \cdot \boldsymbol{r}_{j}} \underbrace{\int U_{j}^{a}(\boldsymbol{r}) e^{-i \boldsymbol{s} \cdot \boldsymbol{r}} d r}_{f_{j}(\boldsymbol{s})},
$$

where $f_{j}(s)$ is the atomic form factor accounting for the specific shape of the atomic potential. In the far field, the situation simplifies, as the scattered spherical wave resembles a plane wave. In other words, the resulting signal will be the Fourier transform of the crystal structure giving rise to strong Bragg reflexes if the exponent $s \cdot \boldsymbol{r}_{j}$ is a multiple of $2 \pi$. This motivates the definition of a reciprocal vector $\boldsymbol{G}_{h k l}$

$$
\boldsymbol{G}_{h k l}=h \boldsymbol{a}_{1}^{*}+k \boldsymbol{a}_{2}^{*}+l \boldsymbol{a}_{3}^{*}
$$

where $h, k$ and $l$ are integer numbers and $\boldsymbol{a}_{1}^{*}, \boldsymbol{a}_{2}^{*}$ and $\boldsymbol{a}_{3}^{*}$ are reciprocal lattice vectors spanning the space of the Fourier transformed lattice, defined by

$$
\boldsymbol{a}_{i}^{*} \cdot \boldsymbol{a}_{j}=2 \pi \delta_{i j} \text { for } i, j=1,2,3
$$

In a crystal with a monoatomic basis, the condition $\boldsymbol{s} \cdot \boldsymbol{r}_{j}$ is fulfilled if the scattering vector $\boldsymbol{s}$ equals a reciprocal vector $\boldsymbol{G}_{h k l}$

$$
\boldsymbol{k}_{\mathrm{out}}-\boldsymbol{k}_{\mathrm{in}}=\boldsymbol{s}=\boldsymbol{G}_{h k l},
$$

where $\boldsymbol{k}_{\text {in }}$ and $\boldsymbol{k}_{\text {out }}$ denote the incoming and outgoing wave vector, respectively. Equation 1.50 is often referred to as the Laue condition, which can be shown to be equivalent to the Bragg condition (Equation 1.43). The measured intensity of the outgoing plane wave for identical scatterers is given by the interference function

$$
I(s)=|A(s)|^{2}=|f(s)|^{2} \sum_{i, j} e^{i s \cdot\left(r_{i}-r_{j}\right)} .
$$


For a crystal with a polyatomic basis, the diffracted amplitude takes the form

$$
\begin{aligned}
A(\boldsymbol{s}) & =\sum_{m, n} f_{m} e^{i \boldsymbol{s} \cdot\left(\boldsymbol{R}_{n}+\boldsymbol{t}_{m}\right)} \\
& =\underbrace{\sum_{m} f_{m} e^{i \boldsymbol{s} \cdot t_{m}}}_{F(\boldsymbol{s})} \underbrace{\sum_{n} e^{i \boldsymbol{s} \cdot \boldsymbol{R}_{n}}}_{G(\boldsymbol{s})},
\end{aligned}
$$

where $\boldsymbol{R}_{n}$ denotes the $\mathrm{n}^{\text {th }}$ unit cell and $f_{m}$ the atomic scattering factor associated with the strength of the $\mathrm{m}^{\text {th }}$ scatterer within the set of basis atoms $\left\{\boldsymbol{t}_{m}\right\}$. The first sum is also known as the structure factor $F(s)$ which determines the magnitude of a Bragg peak, whereas the second term $G(s)$ is associated with the appearance of Bragg reflexes in specific directions according to Laue's condition.

\subsubsection{Surface Lattice}

In LEED, only the top few layers contribute to the diffraction intensity. It is therefore often sufficient to consider a two-dimensional Bravais lattice spanned by

$$
\boldsymbol{R}=n_{1} \boldsymbol{a}_{1}+n_{2} \boldsymbol{a}_{2}
$$

where $n_{1}$ and $n_{2}$ are integers and the unit cell is constructed by $\boldsymbol{a}_{1}$ and $\boldsymbol{a}_{2}$. Accordingly, the reciprocal vector is obtained via

$$
\boldsymbol{G}_{h k}=h \boldsymbol{a}_{1}^{*}+k \boldsymbol{a}_{2}^{*}
$$

with $\boldsymbol{a}_{1}^{*}$ and $\boldsymbol{a}_{2}^{*}$ being the corresponding reciprocal lattice vectors in two dimensions.

The configuration of surface atoms can deviate from that of a bulk plane due to surface reconstructions, the formation of superstructures, like in CDW systems, or adsorbates, resulting in a new periodicity. In general, the surface structure therefore has different lattice vectors $\boldsymbol{b}_{1}$ and $\boldsymbol{b}_{2}$ that can be expressed in terms of the substrate lattice vectors

$$
\boldsymbol{G}_{h k}=\left(\begin{array}{l}
\boldsymbol{b}_{1} \\
\boldsymbol{b}_{2}
\end{array}\right)=\left(\begin{array}{ll}
m_{11} & m_{12} \\
m_{21} & m_{21}
\end{array}\right)\left(\begin{array}{l}
\boldsymbol{a}_{1} \\
\boldsymbol{a}_{2}
\end{array}\right) .
$$

Emerging superstructures may be commensurate or incommensurate with the bulk periodicity resulting in rational or irrational matrix elements $m_{i j}$, respectively. Both scenarii are realized in different CDW phases of the layered transition metal dichalcogenide $1 T-\mathrm{TaS}_{2}$.

Alternatively, Wood's notation is a second common way to define superstructures. It considers the ratios $p=b_{1} / a_{1}$ and $q=b_{2} / a_{2}$ between bulk and surface lattice vectors, and rotation of the superstructure cell by an angle $\phi$. A superstructure is then defined by $(p \times q) R \phi$. This notation, 
however, cannot cover all possible supercell configurations since it assumes the same rotation angle of both $\mathbf{b}_{1}$ and $\mathbf{b}_{2}$. [131, 132].

The reciprocal lattice vectors of the superstructure are defined accordingly by

$$
\left(\begin{array}{l}
\mathbf{b}_{1}^{*} \\
\mathbf{b}_{2}^{*}
\end{array}\right)=M^{*}\left(\begin{array}{l}
\mathbf{a}_{1}^{*} \\
\mathbf{a}_{2}^{*}
\end{array}\right) \text {. }
$$

The two matrices $M$ and $M^{*}$ are related via inversion, yielding

$$
M^{*}=\left(\begin{array}{ll}
m_{11}^{*} & m_{12}^{*} \\
m_{21}^{*} & m_{21}^{*}
\end{array}\right)=\frac{1}{m_{11} m_{22}-m_{21} m_{12}} \cdot\left(\begin{array}{cc}
m_{22} & -m_{21} \\
-m_{12} & m_{11}
\end{array}\right) .
$$

In many cases, the superstructure exhibits a larger periodicity than the bulk structure. In a diffraction experiment, this results in satellite spots around or between reflexes associated with the substrate.

\subsubsection{Ewald Construction}

The Ewald construction visualizes the angular features of elastic diffraction from a lattice, taking into consideration energy and momentum conservation. For diffraction from a two-dimensional lattice, the Laue conditions read

$$
\begin{aligned}
& \left|\boldsymbol{k}_{\text {out }}\right|=\left|\boldsymbol{k}_{\text {in }}\right| \\
& \boldsymbol{s}_{\|}=\boldsymbol{k}_{\text {out }, \|}-\boldsymbol{k}_{\text {in, },}=\boldsymbol{G}_{h k} .
\end{aligned}
$$

The first equation incorporates the conservation of electron energy and determines the radius of the Ewald sphere (see Fig. 1.19). The second equation ensures in-plane momentum conservation. More specifically, for constructive interference, the scattering vector's component parallel to the surface must coincide with an in-plane reciprocal lattice vector $\boldsymbol{G}_{h k}$. The reciprocal lattice of a strictly two-dimensional surface structure can be represented in three dimensions as infinite rods that are oriented perpendicular to the surface plane (see Fig. 1.19). The two-dimensional case can be seen as the limit of a $3 \mathrm{~d}$ lattice where the basis vector $\boldsymbol{a}_{3}$, pointing perpendicular to the surface, extends to an infinite length, resulting in an infinitesimal reciprocal vector $\boldsymbol{a}_{3}^{*}$. Hence, the reciprocal lattice in this dimension becomes the continuum that is expressed by rods. Diffraction reflexes are now given by the intersection points of these rods with the Ewald sphere. In contrast to diffraction from a $3 \mathrm{~d}$ lattice, the Laue condition can always be fulfilled independent of the angle of incidence on the sample [130].

In real systems, the electron beam has a non-zero penetration depth and usually probes more than a single atomic layer. The additional weak out-of-plane periodicity now has to be considered in 
the Laue conditions (Equations 1.59 and 1.60). This leads to additional interference paths between layers, resulting in energy-dependent intensity modulations along the diffraction rods (see Fig. $1.19 \mathrm{~b}$ ), also named LEED oscillations. In this work, we observe an energy-dependent sensitivity of main lattice reflexes to the PLD in $1 T-\mathrm{TaS}_{2}$.
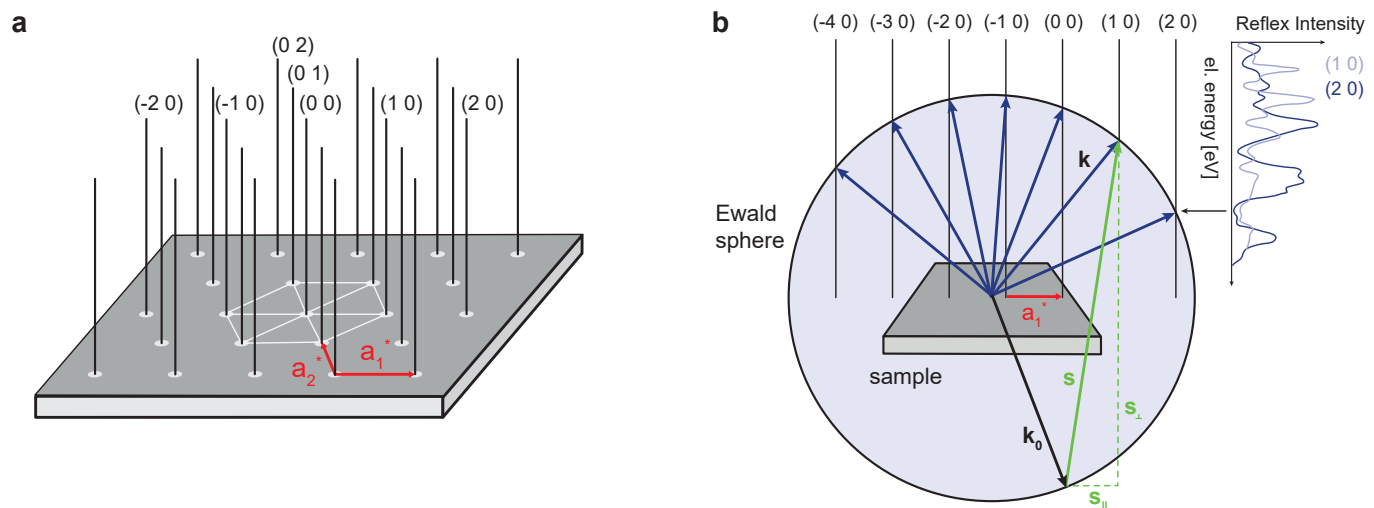

Figure 1.19: Diffraction on a surface crystal. (a) Rods of a hexagonal surface structure in reciprocal space. The spacing is given by the reciprocal basis vectors $\boldsymbol{a}_{1}^{*}$ and $\boldsymbol{a}_{2}^{*}$. (b) Ewald construction for nearnormal incidence scattering. Intersection points of the Ewald sphere with the rods determine the wave vector of scattering reflexes. Particular LEED characteristics are large scattering angles, a large out-of-plane component of the scattering vector $s$ and reflex intensity modulations along the rods when changing the incident electron energy. Adapted from Ref. [130].

\subsubsection{Finite 2d Sheet}

Up to this point, we considered diffraction from infinite bulk and surface lattices. In a real diffraction experiment, however, just a limited number of scatterers will contribute to the diffraction amplitude due to a finite electron beam diameter.

For a monoatomic 1d lattice (and $f(\boldsymbol{s})=1$ ) with scatterers at $\boldsymbol{r}=n \boldsymbol{a}, n=0,1,2, \ldots, N-1$, the diffraction amplitude is given by

$$
A(s)=G(s)=\sum_{n} e^{i \boldsymbol{s}_{x} n \boldsymbol{a}}=\frac{1-e^{i \boldsymbol{s}_{x} N \boldsymbol{a}}}{1-e^{i \boldsymbol{s}_{x} \boldsymbol{a}}} .
$$

The diffraction intensity is the square modulus of the amplitude, yielding

$$
I_{N}\left(s_{x}\right)=\frac{\sin ^{2}\left(\frac{N s_{x} a}{2}\right)}{\sin ^{2}\left(\frac{s_{x} a}{2}\right)} .
$$

As a result, diffraction reflexes are not described by a $\delta$-comb, but by the so-called Laue function featuring maxima for $s_{x}=\left(\frac{2 \pi n}{a}\right)$ (Laue condition) whose width and height scale with $1 / N$ and 
$N^{2}$, respectively. In other words, the limitation of the diffraction volume induces a broadening of diffraction reflexes in comparison to the delta peaks of an idealized infinite lattice. In typical electron diffraction experiments with beam diameters ranging from a few nanometers to several tens of micrometers, this broadening effect is expected to be negligible because a large number of scatterers are involved. More significant broadening contributions are introduced by the electron source or finite correlation lengths in the sample.

\subsubsection{Domains and Defects}

Frequently, periodic superstructures are assembled of larger units, i.e., steps or antiphase domains [131], introducing a further periodicity in the system. For example, Figure 1.20 shows a $1 \mathrm{~d}$ chain with two domains each consisting of $\mathrm{N}$ atoms. The domains are defined by a lattice vector

$$
\boldsymbol{g}=(N+\xi) \boldsymbol{a},
$$

where $\xi$ denotes the spacing between the domains. Consequently, both periodicities are considered in the total diffraction intensity being composed of two Laue functions, yielding [123]

$$
I_{\mathrm{tot}}(s)=I_{N}(\boldsymbol{s} \boldsymbol{a}) \cdot I_{2}(\boldsymbol{s} \boldsymbol{g})=\frac{\sin ^{2}\left(N \frac{\boldsymbol{s} a}{2}\right)}{\sin ^{2}\left(\frac{s \boldsymbol{a}}{2}\right)} \cdot \frac{\sin ^{2}(\boldsymbol{s} \boldsymbol{g})}{\sin ^{2}\left(\frac{s \boldsymbol{g}}{2}\right)} .
$$

As a result, the diffraction pattern exhibits richer features because of an additional finer reciprocal grid. This effect can be observed in the nearly commensurate phase of $1 T-\mathrm{TaS}_{2}$ whose CDW state has an inhomogeneous phase distribution consisting of domains of nearly constant phase separated by regions of rapidly changing phase.

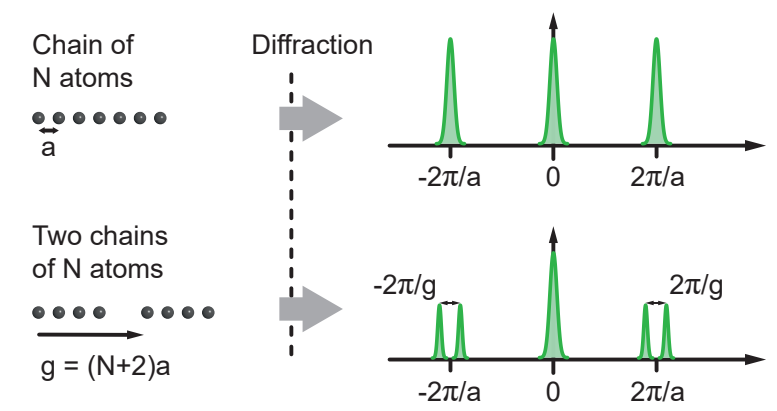

Figure 1.20: Diffraction intensity for a chain of atoms (top) and a cluster of chains (bottom). The additional superstructure leads to a finer grid of diffraction reflexes. Adapted from Ref. [123].

Furthermore, irregular deviations from perfect crystalline periodicity and arrangements of different kinds of defect structures have a characteristic impact on the diffraction pattern. For example, 
randomly distributed adsorbate atoms lead to a non-uniform structure factor for each unit cell, leading to an average scattering intensity given by

$$
\langle I(s)\rangle=\sum_{m, n}\left\langle F_{m} F_{n}^{*}\right\rangle e^{i s \cdot\left(\boldsymbol{r}_{m}-\boldsymbol{r}_{n}\right)}=N\left(\left\langle F^{2}\right\rangle-\langle F\rangle^{2}\right)+\langle F\rangle^{2} \sum_{m, n} e^{i s \cdot\left(\boldsymbol{r}_{m}-\boldsymbol{r}_{n}\right)} .
$$

Here, the diffraction pattern consists of two contributions. The first is a constant part independent of the scattering vector $s$ creating a homogeneous background in the diffraction pattern and the second is a term that is identical to the lattice factor $G(s)$ of the ideal surface scaled by a factor $\langle F\rangle^{2}$. Hence, the reflexes appear on top of a constant background, but have an unaltered intensity profile. Experimentally, that explains the need for clean samples and ultra-high vacuum conditions.

The diffraction spot profile is affected by the size or density distribution within a probed ensemble, such as stepped surfaces, domains or islands of different extent, lattice impurities or dislocation-type defects in a CDW [14]. When modeling, a common choice is to assume a geometrical distribution that allows for a closed calculation. The probability $P$ of finding a domain of size $N$, assuming an average size of $1 / \gamma$, is given by [123]

$$
P(N)=\gamma(1-\gamma)^{N-1},
$$

yielding an exponential distribution in the continuous case. In diffraction, the Fourier-transformed exponential pair correlation function leads to intensity profiles given by a Lorentzian function [123, 131, 133]. This result is reflected in the spot profile analysis for the system investigated in this thesis (see Fig. 3.4).

\subsubsection{Effect of Temperature}

Besides static imperfections of the surface, also dynamic effects due to a finite temperature play an important role in electron diffraction. Temperature-induced atomic vibrations around their equilibrium positions lead to a reduction of reflexes, the so-called Debye-Waller effect, and a thermal diffuse background.

Lattice vibrations produce a time-dependent change of the lattice coordinates $\boldsymbol{r}_{j}$

$$
\boldsymbol{r}_{j}(t)=\boldsymbol{r}_{j}+\boldsymbol{u}_{j}(t)
$$

with time-dependent deviations from the equilibrium positions $\boldsymbol{u}_{j}$. For identical scatterers, the time-averaged diffraction intensity reads

$$
\langle I(\boldsymbol{s})\rangle=|f(\boldsymbol{s})|^{2} \sum_{i, j} e^{i s \cdot\left(\boldsymbol{r}_{i}-\boldsymbol{r}_{j}\right)}\left\langle e^{i s \cdot\left(\boldsymbol{u}_{i}-\boldsymbol{u}_{j}\right)}\right\rangle .
$$


The time average of the exponential term can be expanded for small deviations, yielding

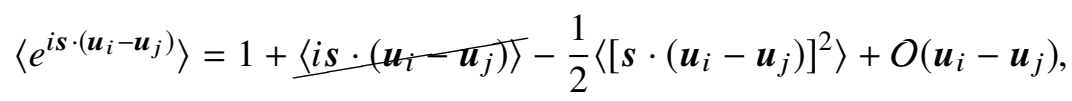

where the linear term vanishes having zero mean. Skipping the details of the derivation [131], the time-averaged intensity for independent oscillators becomes

$$
\langle I(\boldsymbol{s})\rangle=e^{-\left\langle(\boldsymbol{s} \cdot \boldsymbol{u})^{2}\right\rangle}|f(\boldsymbol{s})|^{2} \sum_{i, j} e^{i \boldsymbol{s} \cdot\left(\boldsymbol{r}_{i}-\boldsymbol{r}_{j}\right)}+N|f(\boldsymbol{s})|^{2}\left\{1-e^{-\left\langle(\boldsymbol{s} \cdot \boldsymbol{u})^{2}\right\rangle}\right\} .
$$

The first term describes the usual Bragg diffraction, reduced by an additional factor $e^{-2 W}=e^{-\left\langle(\boldsymbol{s} \cdot \boldsymbol{u})^{2}\right\rangle}$, known as the Debye-Waller factor [131, 134, 135], whereas the second term corresponds to onephonon diffuse scattering. For isotropic displacements and assuming the Debye model for phonons [125], the exponent of the Debye-Waller factor can be written as

$$
\left\langle(\boldsymbol{s} \cdot \boldsymbol{u})^{2}\right\rangle=\boldsymbol{s}^{2}\left\langle\boldsymbol{u}^{2}\right\rangle\left\langle\cos ^{2}(\alpha)\right\rangle=\frac{1}{6} \boldsymbol{s}^{2}\left\langle\boldsymbol{u}^{2}\right\rangle \sim \boldsymbol{s}^{2} \frac{T}{\theta_{D}^{2}},
$$

where $T$ denotes the temperature, $\alpha$ the angle between the scattering vector $s$ and the displacement vector $\boldsymbol{u}$, and $\theta_{D}$ the Debye temperature, a measure for the rigidity of the lattice with respect to vibrations [125]. Large momentum transfer and increased temperatures lower the magnitude of reflexes, while increasing the amount of diffuse signal in the areas in-between. Note that the effect of temperature amounts to a lowering of the scattered spot intensity, but does not change the shape or width.

Moreover, diffuse scattering generally depends on the product of the momentum transfer $s$ and the orientation of the displacement vector $\boldsymbol{u}$, which is determined by the polarization of the involved phonon modes. Therefore, the emerging structure of the diffuse background contains precious information about lattice vibrations in the system. A common, more general approach considers the one-phonon scattering intensity given by [136-138]

$$
I(s) \sim \sum_{j} \frac{\left(n_{j, k}+\frac{1}{2}\right)}{\omega_{j, k}} \underbrace{\left|\sum_{p} \frac{f_{p}}{\sqrt{\mu_{p}}} e^{-M_{p}}\left(\boldsymbol{s} \cdot \hat{\boldsymbol{e}}_{j, p, s}\right)\right|^{2}}_{\left|F_{j}(\boldsymbol{s})\right|^{2}} .
$$

The first sum is taken over all phonon branches, $j$, while the second sums over all atoms $p$ in the unit cell. $n_{j, k}$ is the population of the phonon mode with a momentum $k$, frequency $\omega_{j, k}$ and polarization $\hat{\boldsymbol{e}}_{j, p, \boldsymbol{s}} . f_{p}$ is the atomic scattering factor, $e^{-M_{p}}$ the atomic Debye-Waller factor, and $\mu_{p}$ the atomic mass. In principle, this expression allows to predict the intensity distribution of the diffuse background in reciprocal space, based on the knowledge of $n_{j, k}, \omega_{j, k}$ and $\hat{\boldsymbol{e}}_{j, p, s}$. 
While many different probes, such as angle-resolved photoemission spectroscopy [139], and Raman spectroscopy [140] are available to investigate vibrational modes, recently, a number of groups [141-143] successfully implemented a time-resolved technique based on high-energy electron scattering in order to map the transient changes of diffuse inelastic scattering in momentum space [138, 141]. Exemplarily, Figure 1.21 illustrates these changes in a sequence of snapshots on a picosecond scale, featuring characteristic patterns that belong to specific non-equilibrium phonon distributions in graphite [138].
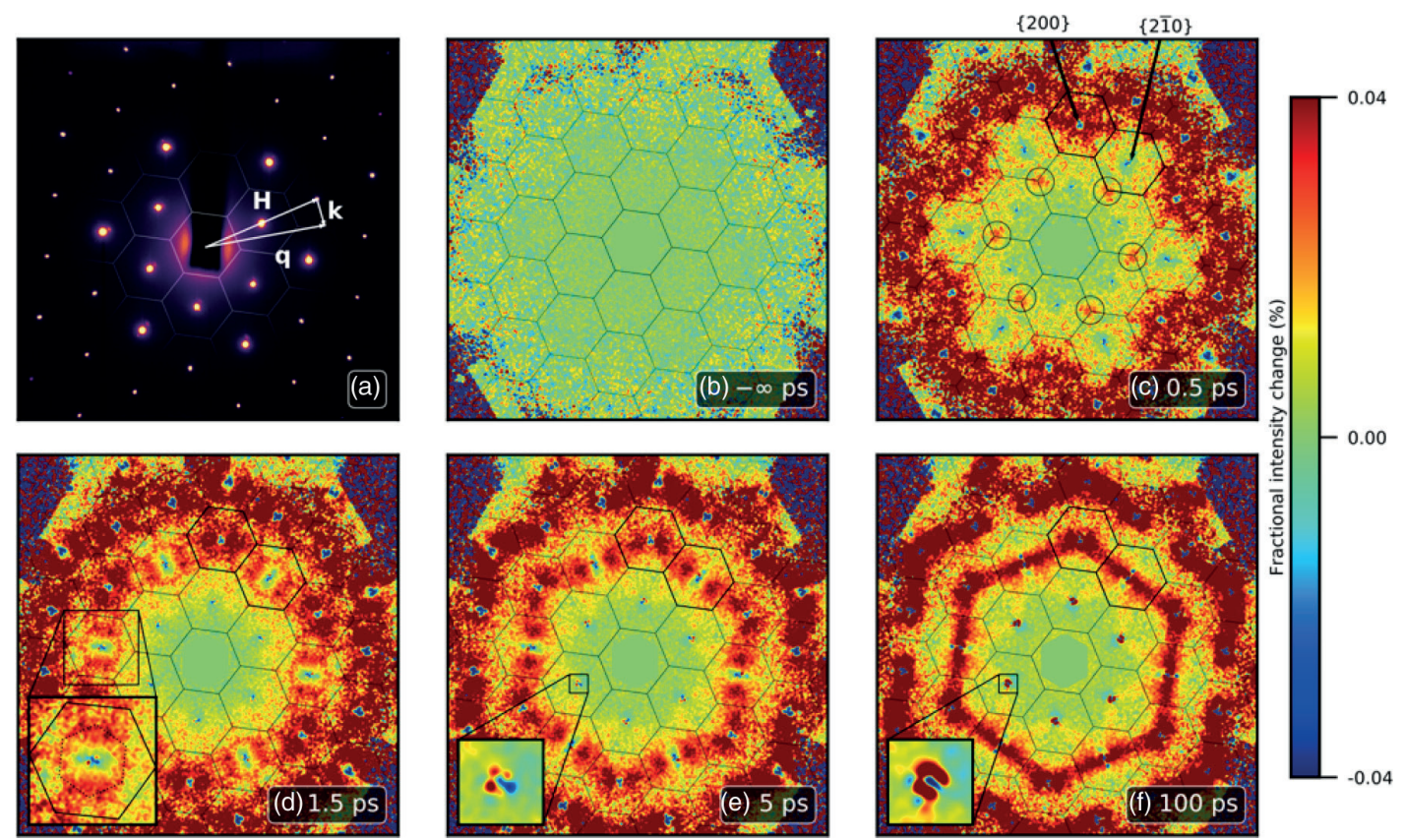

Figure 1.21: Temporal evolution of thermal diffuse background of graphite upon fs-laser excitation. The diffuse background exhibits a rich pattern (red areas) that evolves on a time scale of $100 \mathrm{ps}$. This allows to track the energy relaxation pathways and the equilibration process of different phonon modes. Reprinted figure with permission from Ref. [138] by Stern et al. Copyright 2020 by the American Physical Society.

\subsubsection{Modulated Structures}

The effect of a displacive lattice modulation on a diffraction pattern has already been studied over a century ago in the context of optical gratings [144]. A sinusoidal variation of the grating's spacing resulted in equally spaced satellites, so-called Geister, that decrease in intensity for increasing distance from the main reflex. A crystal structure with displacive lattice modulation was first studied by Dehlinger in 1927 using x-ray beams [145]. The same concept applies for the PLD of CDW materials. In this section, we briefly derive an expression for the structure factor of a periodically modulated lattice. 
For a monoatomic $\left(f_{m}=1\right)$ CDW crystal with equilibrium sites $\{\boldsymbol{L}\}$, the PLD can often be modeled as a single harmonic

$$
\boldsymbol{u}^{0}(\boldsymbol{L})=\boldsymbol{A} \cdot \sin \left(\boldsymbol{Q} \cdot \boldsymbol{L}+\varphi_{\boldsymbol{L}}\right)
$$

The scattering amplitude (equation 1.53) then yields

$$
A(\boldsymbol{s})=\sum_{\boldsymbol{L}} e^{i \boldsymbol{s} \cdot\left(\boldsymbol{L}+\boldsymbol{u}^{0}(\boldsymbol{L})\right)}=\sum_{L} e^{i \boldsymbol{s} \cdot \boldsymbol{L}} e^{i \boldsymbol{s} \cdot \boldsymbol{A} \sin (\boldsymbol{Q} \cdot \boldsymbol{L})},
$$

where $\varphi_{L}$ was set to zero. This expression can be simplified using the Jacobi-Anger expansion for Bessel functions [94]

$$
e^{i z \cdot \sin (\alpha)}=\sum_{n=0}^{\infty} e^{i n \alpha} J_{n}(z),
$$

where $n$ denotes an integer number. Accordingly, the amplitude becomes

$$
A(\boldsymbol{s})=\sum_{\boldsymbol{L}, n} \underbrace{J_{n}(\boldsymbol{Q} \cdot \boldsymbol{A})}_{F(\boldsymbol{s})} e^{i(\boldsymbol{s}+n \boldsymbol{Q}) \cdot \boldsymbol{L}} .
$$

The allowed Bragg reflexes are determined by the altered Laue condition $\boldsymbol{s}=\boldsymbol{G}-n \boldsymbol{Q}$. The main lattice reflections are denoted by $n=0$, while additional CDW satellite peaks decorate the main reflexes, corresponding to integers $n=1,2, \ldots$ The structure factor $F(s)$ determines the brightness of each order and follows the individual Bessel function of $n^{\text {th }}$ order.

For incommensurate crystals, the sum over the lattice $\{\boldsymbol{L}\}$ cannot be restricted to a single unit cell since, strictly speaking, the latter does not exist. Nevertheless, the structure factor can be defined in a similar way, as the scattering normalized to a particular volume [70]. For a triple-CDW, the above derivation can be naturally extended and yields satellite peaks in three orientations as shown in the static LEED diffraction patterns of the NC and IC CDW phase of $1 T-\mathrm{TaS}_{2}$ (Fig. 2.4, 3.2, 3.8 and 3.9).

\subsubsection{Effects of CDW Collective Excitations}

It is important to note that the typical modes of an incommensurate structure, amplitudons and phasons, do not increase the number of degrees of freedom. It remains the value of three times the number of atoms in the crystal [146]. Since phasons are CDW-coupled modes with a completely softened dispersion, they can have large amplitudes similar to low-energy acoustic phonons that are known to contribute to the normal Debye-Waller factor. In real materials, damping and the size of a potential phason gap also play a role [70]. The additional low-frequency modes that coexist and merge with the normal acoustic phonons, affect many physical properties, such as the low-temperature heat capacity, electrical resistivity, NMR spectrum and lattice thermal conductivity 
[95]. In this section, we discuss theoretically the influence of collective modes on the diffraction pattern.

In 1971, Overhauser [94] first predicted that incommensurate CDW systems should have a new dynamical mode that corresponds to the phase fluctuations of the modulation wave in space and time. For a monoatomic crystal with a sinusoidal equilibrium modulation (Equation 1.73), phasons are assumed to be the long-wavelength fluctuations in the phase $\varphi_{L}$ of the modulation. The dynamic distortion of atoms in one phason mode is then given by

$$
\begin{aligned}
\boldsymbol{u}(t) & =A \sin \left[\boldsymbol{Q} \cdot \boldsymbol{L}+\varphi_{\boldsymbol{q}} \sin (\boldsymbol{q} \cdot \boldsymbol{L}-\omega t)\right] \\
& \approx u^{0}+\frac{1}{2} A \varphi_{\boldsymbol{q}}(\sin [(\boldsymbol{q}+\boldsymbol{Q}) \cdot \boldsymbol{L}-\omega t]+\sin [(\boldsymbol{q}-\boldsymbol{Q}) \cdot \boldsymbol{L}-\omega t]),
\end{aligned}
$$

where $\varphi_{\boldsymbol{q}}$ denotes the amplitude of the particular phason mode and $\omega$ its frequency. In this approximation, a phason mode constitutes a coherent superposition of phonon modes with wave vectors $\boldsymbol{Q}+\boldsymbol{q}$ and $\boldsymbol{Q}-\boldsymbol{q}$. In reciprocal space, this implies that phasons are located near satellite reflexes, creating an additional diffuse background [147, 148]. According to Overhauser and Giuliani [94, 95], phasons and amplitudons lead to additional Debye-Waller-type structure factors given by

$$
F_{m}^{\varphi}=e^{-m^{2}\left\langle\delta \varphi^{2}\right\rangle}
$$

and

$$
F_{m}^{A}=e^{-m(m-1)\left\langle\delta A^{2}\right\rangle},
$$

where $m \geq 0$ denotes the reflex order (main lattice: $m=0$, CDW satellite: $m>0$ ), and the quantities $\left\langle\delta \varphi^{2}\right\rangle$ and $\left\langle\delta A^{2}\right\rangle$ are the mean square deviation of phase and amplitude, respectively. These structure factors only affect the satellite intensities and do not depend on the scattering vector, in contrast to the conventional Debye-Waller factor [95, 148]. Moreover, Overhauser stated that phason excitations might even reduce the intensity of CDW satellites below the limit of observability [94].

Axe [149] reevaluated Overhauser's expression and concluded that the phason contribution had been strongly overestimated, which is supported by the observation of satellite reflexes in experiments [68]. First, he reproduces Overhausers's result and compares two versions of the Gaussian phase approximation (GPA). The structure factor for a monoatomic crystal with a cosinusoidal modulation is given by

$$
F(\boldsymbol{s})=\sum_{\boldsymbol{L}} e^{i s \cdot \boldsymbol{L}}\left\langle e^{i s \cdot \boldsymbol{u}_{\boldsymbol{L}}}\right\rangle
$$

with

$$
u_{\boldsymbol{L}}=A \cos \left(\theta_{\boldsymbol{L}}-\varphi_{\boldsymbol{L}}\right), \quad \theta_{\boldsymbol{L}}=\boldsymbol{Q} \cdot \boldsymbol{L}-\theta_{0}
$$


where $\theta_{0}$ is a global phase. In the GPA, it is assumed that the phase $\varphi_{\boldsymbol{L}}$ fluctuates with a Gaussian distribution. The average value of a phase-dependent function is then defined by

$$
\left\langle f\left(\varphi_{\boldsymbol{L}}\right)\right\rangle \sim \int f\left(\varphi_{\boldsymbol{L}}\right) e^{-(1 / 2)\left(\varphi_{\boldsymbol{L}}^{2} /\left\langle\varphi_{\boldsymbol{L}}^{2}\right\rangle\right)} d \varphi_{\boldsymbol{L}}
$$

In this approximation, the structure factor becomes

$$
F(\boldsymbol{s})=\Delta(\boldsymbol{s}+m \boldsymbol{Q}) \sum_{m} i^{m} e^{-i m \theta_{0}} \boldsymbol{J}_{m}(\boldsymbol{s} \cdot A) e^{-m^{2}\left\langle\varphi^{2}\right\rangle / 2},
$$

where $\Delta$ denotes a comb of delta functions representing the Laue condition for main and satellite reflexes. Axe's expression of the structure factor is in agreement with Overhauser's result, in which phase fluctuations are independent of the position. However, phase fluctuations cause larger position fluctuations near the nodes of the modulation wave than near maxima. Consequently, the mean square position fluctuations are modulated in space with a wave vector of $2 \boldsymbol{Q}$.

In a second GPA version, Axe splits the displacement modulation into the average displacement and its deviation in order to account for a thermal smearing of individual atoms. This yields

$$
\left\langle\boldsymbol{u}_{\boldsymbol{L}}\right\rangle=\boldsymbol{A}\langle\cos (\varphi)\rangle \cos \left(\theta_{\boldsymbol{L}}\right)+\boldsymbol{A}\left\langle\sin \left(\varphi_{\boldsymbol{L}}\right)\right\rangle \sin \left(\theta_{\boldsymbol{L}}\right)=\boldsymbol{\eta} \cos \left(\theta_{\boldsymbol{L}}\right)
$$

with a renormalized amplitude

$$
\boldsymbol{\eta}=\boldsymbol{A}\langle\cos (\varphi)\rangle
$$

and

$$
\boldsymbol{\delta} \boldsymbol{u}_{\boldsymbol{L}}=\boldsymbol{u}_{\boldsymbol{L}}-\left\langle\boldsymbol{u}_{\boldsymbol{L}}\right\rangle=\boldsymbol{A}\left[\cos \left(\theta_{\boldsymbol{L}}\right)\left(\cos \left(\varphi_{\boldsymbol{L}}\right)-\left\langle\cos \left(\varphi_{\boldsymbol{L}}\right)\right\rangle\right)+\sin \left(\theta_{\boldsymbol{L}}\right) \sin \left(\varphi_{\boldsymbol{L}}\right)\right] .
$$

The structure factor is then given by the expression

$$
F(\boldsymbol{s})=\sum_{\boldsymbol{L}, m} i^{m} e^{i(\boldsymbol{s}+m \boldsymbol{Q}) \cdot \boldsymbol{L}} J_{m}(\boldsymbol{s} \cdot \boldsymbol{\eta})\left\langle e^{i \boldsymbol{s} \cdot \boldsymbol{\delta} \boldsymbol{u}_{\boldsymbol{L}}}\right\rangle .
$$

In this approach, it is important to note that phase fluctuations influence the structure factor via two effects. The amplitude is renormalized and fluctuations around this new average value contribute via the term $\left\langle e^{i \boldsymbol{s} \cdot \boldsymbol{\delta} \boldsymbol{u}_{\boldsymbol{L}}}\right\rangle$. This term does not have simple closed form and is considered in an expansion [149]. Expanding the exponential and the term $\left\langle e^{i s \cdot \delta u_{\boldsymbol{L}}}\right\rangle$ for small $\left\langle\varphi^{2}\right\rangle$ yields Overhauser's result. For larger $\left\langle\varphi^{2}\right\rangle$, both approaches give differing predictions.

Axe provides a third approach for the general case, including amplitudons, in which the displacements are assumed to follow a Gaussian distribution. From Landau theory, two new normal coordinates $A_{q}$ and $\varphi_{q}$ can be introduced. The displacement deviation can then be written as a superposition of amplitude and phase modes, yielding

$$
\boldsymbol{\delta} \boldsymbol{u}_{\boldsymbol{L}}=\frac{1}{\sqrt{2}} \sum_{\boldsymbol{q}}\left[\cos \left(\boldsymbol{q} \cdot \boldsymbol{L}-\theta_{0}\right) \boldsymbol{A}_{\boldsymbol{q}}+\sin \left(\boldsymbol{Q} \cdot \boldsymbol{L}-\theta_{0}\right) \boldsymbol{\varphi}_{\boldsymbol{q}}\right] e^{i \boldsymbol{q} \cdot \boldsymbol{L}}
$$


For independent modes, one obtains the mean square displacement

$$
\left\langle\boldsymbol{\delta} \boldsymbol{u}_{\boldsymbol{L}}^{2}\right\rangle=\left\langle\boldsymbol{\delta} \boldsymbol{\eta}_{\boldsymbol{A}}^{2}\right\rangle \cos ^{2}\left(\theta_{\boldsymbol{L}}\right)+\left\langle\boldsymbol{\delta} \boldsymbol{\eta}_{\boldsymbol{\varphi}}^{2}\right\rangle \sin ^{2}\left(\theta_{\boldsymbol{L}}\right)
$$

where $\left\langle\delta \boldsymbol{\eta}_{\boldsymbol{A}}^{2}\right\rangle$ and $\left\langle\boldsymbol{\delta} \boldsymbol{\eta}_{\boldsymbol{\varphi}}^{2}\right\rangle$ denote the mean square position fluctuations associated with amplitude and phase fluctuations, respectively. For the Gaussian displacement approximation (GDA), the structure factor

$$
F(\boldsymbol{s})=\sum_{n} e^{i \boldsymbol{s} \cdot \boldsymbol{L}} e^{i \boldsymbol{s} \cdot\left\langle\boldsymbol{u}_{\boldsymbol{L}}\right\rangle}\left\langle e^{i \boldsymbol{s} \cdot \delta \boldsymbol{u}_{\boldsymbol{L}}}\right\rangle
$$

becomes

$$
F(\boldsymbol{s})=\Delta(\boldsymbol{s}+m \boldsymbol{Q}) i^{m} e^{-w^{\prime}} \sum_{l}(-1)^{l} J_{m-2 l}(\boldsymbol{s} \cdot \boldsymbol{\eta}) I_{l}\left(w^{\prime \prime}\right)
$$

with

$$
w^{\prime}=\frac{\overline{\boldsymbol{s}}^{2}}{2}\left(\left\langle\boldsymbol{\delta} \boldsymbol{\eta}_{\boldsymbol{A}}^{2}\right\rangle+\left\langle\boldsymbol{\delta} \boldsymbol{\eta}_{\varphi}^{2}\right\rangle\right) \text { and } w^{\prime \prime}=\frac{\overline{\boldsymbol{s}}^{2}}{2}\left(\left\langle\boldsymbol{\delta} \boldsymbol{\eta}_{\boldsymbol{A}}^{2}\right\rangle-\left\langle\boldsymbol{\delta} \boldsymbol{\eta}_{\varphi}^{2}\right\rangle\right)
$$

where $J_{m}$ and $I_{l}$ are (modified) Bessel functions and $\overline{\boldsymbol{s}}=\boldsymbol{s} \cdot \boldsymbol{A} /|\boldsymbol{A}|$. This result is much more complex and deviates from Overhauser's expression. In the regime $\boldsymbol{s} \cdot \boldsymbol{\eta}<1$ and $n^{2}\left\langle\varphi^{2}\right\rangle$, however, the results of GPA and GDA yield identical predictions. For the discussion of other regimes, we refer to Axe's publication [149].

The results published by Overhauser and Axe were reprinted in the books of Bruce et al. [150], Currat and Jansen [146], Krivoglaz [148] and van Smaalen [70]. Several follow-up works, based on either Overhauser's or Axe's approach, illuminate further aspects. Wang and Overhauser [151] report on the diffuse scattering of $\mathrm{x}$-rays by phasons. They derive expressions for the absolute diffraction intensity, surrounding a CDW satellite, and claim that phason scattering dominates other diffuse scattering. Adlhart [152] extends Axe's approach and takes normal phonons into account. He states that phasons are observable near intense satellite reflexes, but also near reflexes of the main lattice, and that amplitudons should be visible near main and satellite reflexes when $(\boldsymbol{Q A})$ is sufficiently large. More recently, Aslayan et al. [153] pointed out weaknesses of Axe's approach, regarding the modes extracted from Landau theory. According to their comment, the new set of variables leads to physical inconsistencies due to anharmonicities in the Landau functional.

Several experimental works claimed evidence for a phason Debye-Waller factor [154-156] and phason diffuse scattering $[147,151]$ using x-ray and electron diffraction techniques. Nevertheless, their results remain inconclusive and do not resolve the quite complex theoretical situation, discussed above [70]. Despite a number of more recent works [36, 38], the role of phasons in diffraction experiments still remains controversial even today. 


\subsection{The layered Compound $1 T-\mathrm{TaS}_{2}$}

As part of the family of transition metal dichalcogenides, the prototypical quasi- $2 \mathrm{~d}$ metallic compound $1 T-\mathrm{TaS}_{2}$ features a particularly rich electronic phase diagram which is spanned by temperature and pressure $[157,158]$. The material is host to a commensurate, a triclinic, a nearly commensurate and an incommensurate charge-density wave state (see Fig. 3.3), first shown by electron diffraction experiments $[42,159]$. At low temperatures, there is evidence for a Mott-insulating $[76,160]$ and a metastable hidden phase [7]. Moreover, below $T \approx 50 \mathrm{~K}$, the system can be transfered into to a superconducting state by applying pressure [157].

Tantalum disulfide is a layered material and comes in various polytypes [159] defined by the stacking sequence of the layers (see Fig. 1.22). The hexagonal unit cell of the polytype $1 T$ consists of a S-Ta-S trilayer in which the tantalum atoms are octahedrally coordinated (purple octahedron in Fig. 1.22) to the neighboring sulfur atoms. Within a trilayer, atoms are covalently bonded, while two trilayers are bound by weaker van der Waals interactions, turning $1 T-\mathrm{TaS}_{2}$ into a quasi-2d material. Above $543 \mathrm{~K}\left(850^{\circ} \mathrm{C}\right)$, in the metallic phase, the structure is classified as the symmetry space group 164-P3m1 $\left(\mathrm{Cd}(\mathrm{OH})_{2}\right.$ type $)$ with lattice parameters $a_{0}=b_{0}=3.36 \AA$ and $c_{0}=5.90 \AA$ [161]. Below this temperature, the structure of the metallic state is distorted due to the formation of different CDW states. In what follows, the structures of these lower-temperature phases will be characterized by the lattice distortions in relation to the structure of the high-temperature metallic phase.

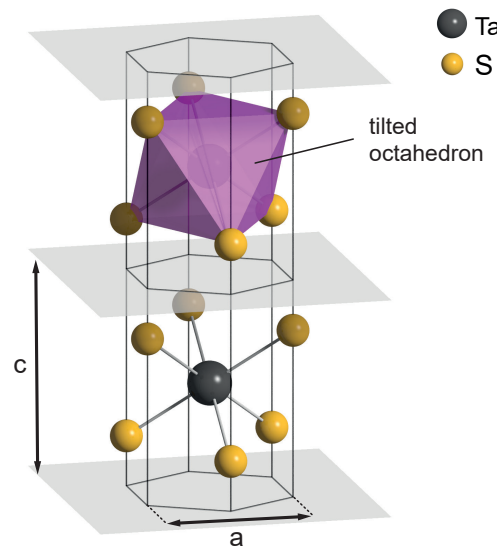

b

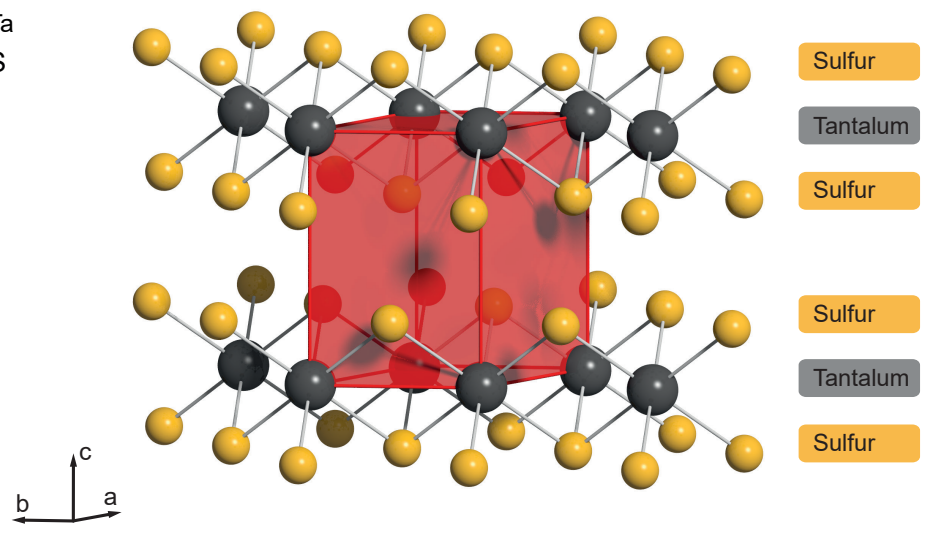

Figure 1.22: Atomic structure of $1 T-\mathrm{TaS}_{2}$. (a) Sulfur atoms are octahedrally (violet) coordinated around a Tantalum site. Adapted from Refs. [72, 158]. (b) The $1 T$ crystal structure is trigonal. A unit cell (red) contains a Tantalum atom $(0,0,0)$ and two sulfur atoms at $\pm\left(\frac{1}{3}, \frac{2}{3}, z\right)$ with $z \approx 0.25$ [161]. There are weak van der Walls bonds between S-Ta-S trilayers. 


\subsubsection{CDW States}

Generally, the emergence of a CDW phase leaves fingerprints in various measurable quantities. For example, satellite peaks appear around the main lattice reflexes in electron diffraction patterns indicating a structural transformation (see Fig. 2.4, 3.2, 3.8 and 3.9). Close to the critical temperature of the phase transition, anomalous behavior is observable in the temperature dependence of the heat capacity, magnetic susceptibility, electrical resistivity or spectroscopic band gaps (see Fig. 1.23) resulting in kinks, divergencies or hysteresis loops.
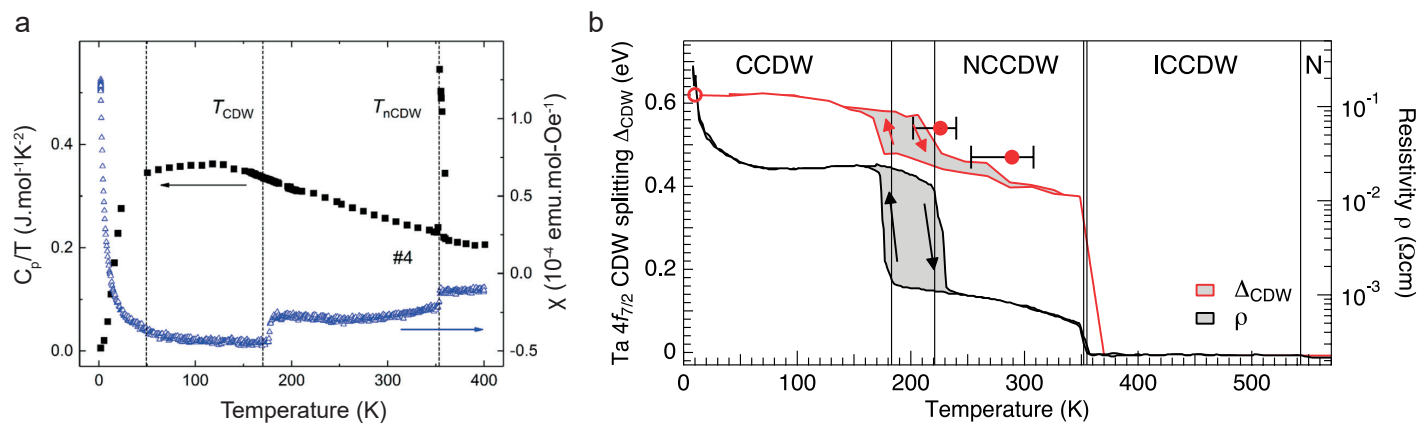

Figure 1.23: Thermodynamic properties of $1 T-\mathrm{TaS}_{2}$. (a) Comparison of the temperature dependence of (a) specific heat (black squares) and magnetic susceptibility (blue triangles) upon cooling [162] as well as (b) the in-plane resistivity [163]. Vertical lines indicate the position of the CDW phase transitions. Adapted from Ref. [162] by Kratochvilova et al., licensed under Creative Commons CC BY, and from [163] by Hellmann et al., licensed under CC BY 3.0.

For $T<543 \mathrm{~K}$, this layered material exhibits a stable triple- $\boldsymbol{Q}$ charge-density wave being described by three superimposed standing waves at a relative angle of $120^{\circ}$ degrees with respect to each other. Besides exhaustive experimental studies, a number of theoretical works based on free-energy potentials have investigated the specific shape of the CDW, as well as its characteristic collective excitations and phase transitions (single CDW: [78, 164, 165], triple CDW: [115, 166, 167]). We will now discuss the three main CDW phases of the material in more detail, starting at lowest temperatures.

\subsubsection{Commensurate Phase}

For temperatures below $183 \mathrm{~K}$, the material is in the commensurate (C) CDW state, which exhibits the charge-density modulation with the simplest geometry. For a single trilayer, the center atoms form a hexagonal lattice while the entire trilayer is part of an effective $\mathrm{P} 3$ space group. The supercell in the C-phase is usually denoted as $\sqrt{13} a \times \sqrt{13} a \times 13 c$ [161] with a rotation of $\phi=13.9^{\circ}$ (see Fig. 
1.24). Clusters of thirteen tantalum atoms form so-called 'stars of David', in each of which twelve Ta atoms move laterally towards the center Ta-atom (see Fig. 1.24). There are two energetically equivalent in-plane configurations considering a clockwise ( $\alpha$-phase) and counterclockwise ( $\beta$ phase) rotation $[159,168]$. The supercell basis (see Sec. 1.2.3.1) vectors within a triple-layer are given by

$$
M_{1}=\left(\begin{array}{cc}
3 & -1 \\
1 & 4
\end{array}\right) \text { and } M_{2}=\left(\begin{array}{cc}
4 & 1 \\
-1 & 3
\end{array}\right) \text {. }
$$

In reciprocal space, diffraction reflexes appear at the positions of the linearly combined CDW wave vectors (for one of the above configurations) $\boldsymbol{Q}_{1, c}=\left(3 \boldsymbol{a}_{0}^{*}+\boldsymbol{b}_{0}^{*}\right) / 13=0.2308 \boldsymbol{a}_{0}^{*}+0.0769 \boldsymbol{b}_{0}^{*}$ and $\boldsymbol{Q}_{2, c}=\left(\boldsymbol{a}_{0}^{*}-4 \boldsymbol{b}_{0}^{*}\right) / 13$ [161]. In order to simplify the theoretical description incorporating the 3 -fold symmetry, a third wave vector $\boldsymbol{Q}_{3, c}$ (triple CDW) is introduced, having an angle of $120^{\circ}$ to $\boldsymbol{Q}_{1, c}$ and $\boldsymbol{Q}_{2, c}$. With a wavelength of $3.606 a_{0}$, the CDW fully registers to the underlying main lattice, resulting in coinciding higher-order diffraction reflexes. In section 1.2.3.6, the emergence of higherorder spots was the result of a harmonic lattice distortion that gives rise to a description via Bessel functions. Any deviations from a purely harmonic distortion, however, can have a similar effect. In several works, Nakanishi and Shiba [115, 165-167] studied the role of additional harmonics using Landau functionals, and pointed out their importance, in particular in the $\mathrm{C}$ and $\mathrm{NC}$ phase.

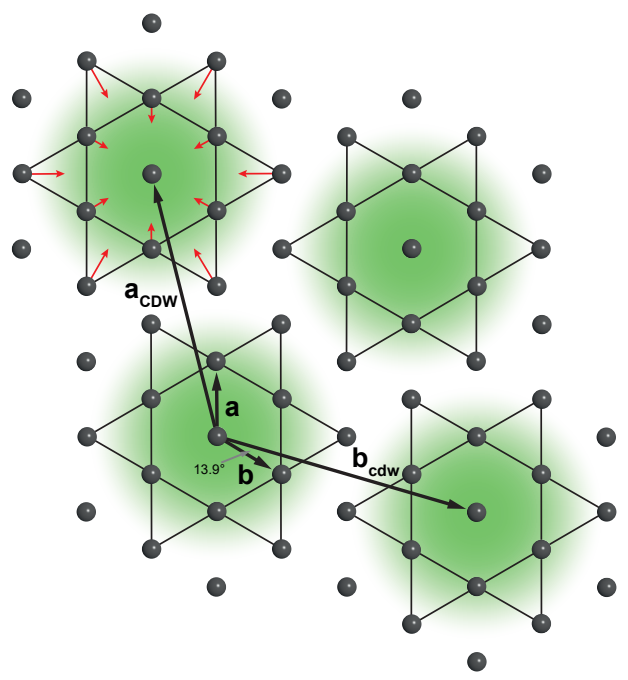

Figure 1.24: Real space unit cell of the commensurate CDW. Clusters of 13 atoms (gray spheres) emerge that are distorted by the charge modulation (red arrows) forming stars. The superstructure is determined by the vectors $\boldsymbol{a}_{\mathrm{CDW}}=3 \boldsymbol{a}-\boldsymbol{b}$ and $\boldsymbol{b}_{\mathrm{CDW}}=\boldsymbol{a}+4 \boldsymbol{b}$ and corresponds to the $\sqrt{13} a \times \sqrt{13} a$ in-plane unit cell.

Moreover, it is important to note that, besides the in-plane formation of stars of David, outof-plane distortions are taking place, leading to a periodic swelling of individual layers (see Fig. 
1.25) $[129,169]$. Tantalum atoms are mainly displaced within the plane, having mean in-plane and out-of-plane distortion amplitudes of $\bar{A}_{\mathrm{Ta}, \|}=0.22 \AA$ and $\bar{A}_{\mathrm{Ta}, \perp}=0.01 \AA$ in the bulk [129], respectively. However, sulfur atoms exhibit the reverse behavior and are mainly displaced in the out-of-plane direction with $\bar{A}_{\mathrm{S}, \|}=0.02 \AA$ and $\bar{A}_{\mathrm{S}, \perp}=0.1 \AA$ [129]. Recently, it was shown [129] that the C-phase surface structure exhibits a distinct on-top-stacked double trilayer periodicity for which neighboring double trilayers are shifted by almost half a superstructure. Figure 1.25 exemplifies this particular stacking using four trilayers.

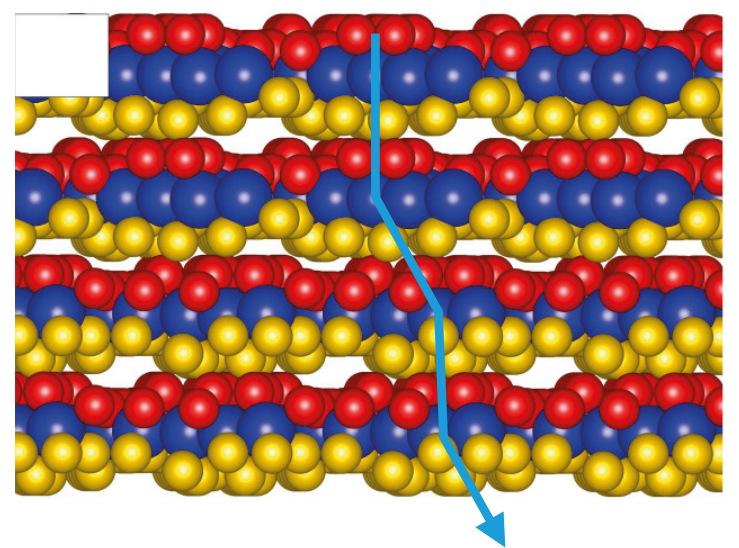

Figure 1.25: Stacking of commensurate CDW. Side view of four S-Ta-S trilayers (red, blue, yellow) in direction of a superstructure unit vector. The blue arrow indicates the stacking sequence of layers. Sulfur atoms (red and yellow) exhibit a strong out-of-plane distortion component. Reprinted figure with permission from Ref. [129] by von Witte et al. Copyright 2020 by the American Physical Society.

\subsubsection{Nearly commensurate Phase}

Heating the system above $223 \mathrm{~K}$ [161] (see Fig. 3.3), the material undergoes a phase transition into the triclinic phase, before it changes to the nearly commensurate (NC) CDW phase at $283 \mathrm{~K}$ (see also Fig. 2.4 and 3.8). As the name NC indicates, it is closely related to the C-phase with its $\sqrt{13} a \times \sqrt{13} a$ structure. This is also reflected in the similar position of the NC-superstructure diffraction peaks. For $T=298 \mathrm{~K}$, the CDW vector becomes $\boldsymbol{Q}_{N C}=0.245 \boldsymbol{a}_{0}^{*}+0.0068 \boldsymbol{b}_{0}^{*}+\boldsymbol{c}_{0}^{*} / 3$ with a temperature-dependent angle of $\phi \approx 12^{\circ}$ [161]. However, the superstructure is not uniform and exhibits commensurate, roughly hexagonally shaped domains that are arranged in a kagome patchwork [157, 161, 170] (see Fig. 1.26). As discussed in chapter 1.1.5.2, the emerging pattern is due to an incommensurate CDW wavevector that is close to the commensurate value, resulting in a local registration to the main lattice to gain commensurability energy, and phase slips to maintain the correct long-range average periodicity of the CDW-lattice [170]. The commensurate domains 
are separated by corner-sharing triangular discommensurate regions $[161,166]$ where the average distortion amplitude is significantly reduced and the CDW phase changes rapidly. Within a domain, the distortion amplitude increases towards the center, leading to more pronounced stars of David [161]. Analogous to the pure C-phase, the same out-of-plane distortion behavior of Ta and S is observed.

Diffraction on such a block-wave distortion modulation with phase jumps at the domain borders [161] results in a fine reciprocal grid (see also section 1.2.3.4) which can be observed in the emergence of groups of higher order satellites (see Fig. 2.4). Consequently, in the triple CDW description, multiple harmonics of the charge-density wave vector $\boldsymbol{Q}_{N C}$ play an important role in order to form the block-wave structure $[115,165,166]$. The density of the fine reciprocal grid depends on the temperature-dependent patch sizes. At room temperature, the average domain diameter corresponds to approximately five stars-of-David clusters and the center-to-center domain distance is approximately $73 \AA$ [161]. The angle of $\boldsymbol{Q}_{\mathrm{NC}}$ also exhibits a temperature dependence, varying from about $13^{\circ}$ at $250 \mathrm{~K}$ to about $11^{\circ}$ at $350 \mathrm{~K}$ [170].

It is difficult to assign a global value for the distortion amplitude, as it is strongly modulated along the structure. Considering that a large part of the volume can be assumed to be commensurate-like, the average distortion value is expected to be only slightly decreased compared to the global C-phase. From Spijkerman et al., precise values of individual atom displacements at room temperature can be extracted, leading to mean values across 100 unit cells of $\bar{A}_{\mathrm{Ta}, \|}=0.1761 \AA, \bar{A}_{\mathrm{Ta}, \perp}=0.0154 \AA$, $\bar{A}_{\mathrm{S}, \|}=0.0324 \AA$ and $\bar{A}_{\mathrm{S}, \perp}=0.064 \AA$.

a

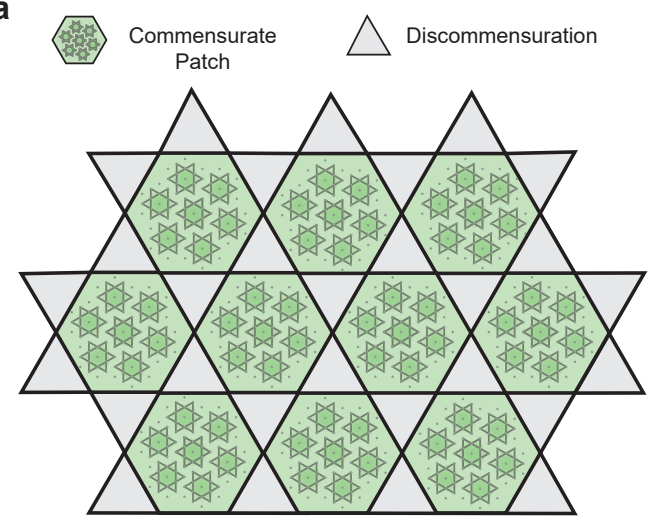

b

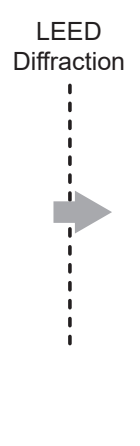

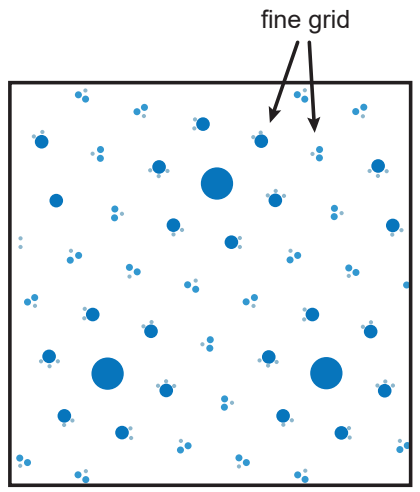

Figure 1.26: Structure of the NC phase. (a) Sketch of a kagome lattice with corner-sharing commensurate patches (green hexaga) that are separated by incommensurate regions or discommensurations (gray triangles). (b) Diffractive probing of the NC structure results in a rich diffraction pattern adding a fine grid of spots. Adapted from Refs. [161] and [56] 


\subsubsection{Incommensurate Phase}

At a temperature of about $353 \mathrm{~K}, 1 T-\mathrm{TaS}_{2}$ shows a first-order phase transition into the incommensurate CDW state, in which the CDW vector $Q_{\mathrm{IC}}=0.283 \boldsymbol{a}_{0}^{*}+\boldsymbol{c}_{0}^{*} / 3$ [161] aligns to the main lattice with a periodicity of $3.53 a_{0}$ [170]. In the LEED diffraction experiment, only first-order satellite spots are observed (see Fig. 3.2 and 3.9), pointing to a weak harmonic distortion modulation. Due to the incommensurate nature of the structure and the size of the electronic gap, the average displacement amplitude is expected to be lower. It was measured by means of electron and x-ray diffraction experiments, adopting bulk distortion values for Ta ranging from $A=0.03 \AA$ [42] up to $0.166 \AA$ [154].

As the CDW is fully incommensurate, it does not register to the underlying lattice, allowing it to freely float across the crystal. This renders the IC-phase of $1 T-\mathrm{TaS}_{2}$ a promising candidate for gapless collective excitations, since very low excitation energies should suffice to populate a major fraction of phase modes (see also Fig. 1.8). However, lattice impurities possibly lead to pinning of the CDW and to the opening of a gap in the phonon band structure of the corresponding low-lying excitations. Generally, the IC-CDW phase is much less studied since reflex intensities are lower due to both a small PLD amplitude and a significant Debye-Waller suppression. In addition, spectroscopic features in the electronic band structure are strongly smeared out in this high-temperature regime. Heating the structure above $543 \mathrm{~K}$ melts the CDW structure and takes the crystal, in a second-order phase transition, into the normal metal state $[72,76]$.

\subsubsection{Electronic and Phonon Band Structures}

The CDW formation is paralleled by significant change of the electronic and phonon band structure. Figure 1.27a shows a sketch of the 2D Brillouin zone of the normal (thick line) and reconstructed commensurate $1 T-\mathrm{TaS}_{2}$ phase with elliptical pockets of the unreconstructed Ta $5 \mathrm{~d}$ Fermi surface at the edges [72]. Red arrows indicate possible nesting vectors rendering the system unstable for a Peierls-type transition. While the Fermi surface topology suggests a Peierls-type scenario for the transition into the IC phase $[159,171]$, the nature of the $\mathrm{C}$ and NC phase, as well as of the IC-NC and NC-C transition are still under debate [172].

In order to investigate the electronic properties of $1 T-\mathrm{TaS}_{2}$, many different techniques have been applied, such as optical scattering [173], electrical transport [174, 175] and angle-resolved photoemission spectroscopy (ARPES) [76]. In ARPES, the IC phase (see 1.27b) displays a single nesting-induced CDW gap $\Delta_{\text {ICCDW }}$ of $300-400 \mathrm{meV}$ between the high symmetry points $\mathrm{M}$ and $\mathrm{K}$, whereas the $\mathrm{C}$ phase exhibits a much richer structure with multiple spectral signatures. At low 
a

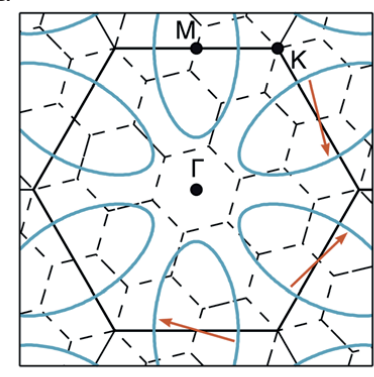

b

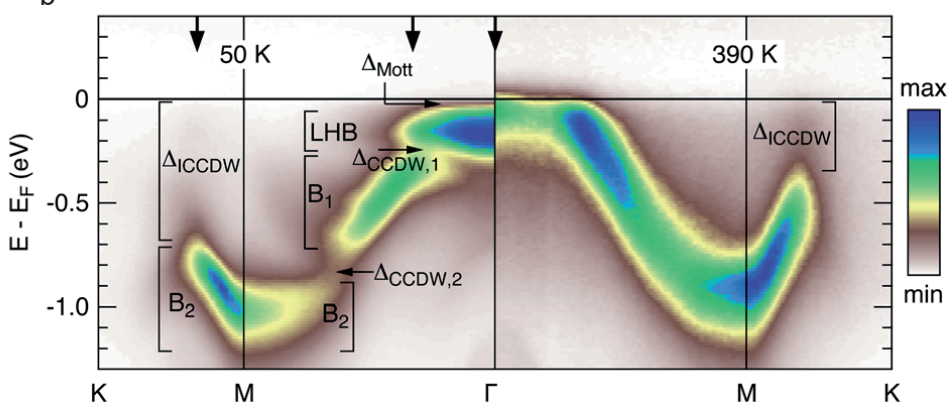

Figure 1.27: (a) Brillouin zones in the normal (thick solid lines) and commensurate (thin dashed lines) phases. The unreconstructed Ta 5d Fermi surface has elliptical pockets. Possible nesting vectors are indicated. (b) Measured ARPES band structure in the commensurate (left) and incommensurate (right) charge-density wave phase. Characteristic spectral signatures are labeled. From Ref. [76] by Sohrt et al., licensed under Creative Commons CC BY.

temperatures, it is commonly accepted that strong electron-electron interactions lead to the opening of a Mott gap with an upper and lower Mott band separated by approximately $100 \mathrm{meV}$, transforming the system into an insulator [76]. The NC phase can be understood as a combination of these two pictures, exhibiting a spatially inhomogeneous pattern of both commensurate and incommensurate regions. However, Ritschel et al. recently challenged the paradigm of local Mott physics as the main driving mechanism behind the formation of these gaps, and proposed an alternative, pointing out the crucial role of the CDW stacking and the related orbital order in the direction perpendicular to the layers $[176,177]$.

As discussed above, the static structural distortion in Peierls systems can be described as a strongly softened and eventually frozen-in phonon mode, a consequence of a drastically altered phonon band structure. The phonon response of $1 T-\mathrm{TaS}_{2}$ was studied around the zone center by means of infrared and Raman spectrocopy [178-182], and over the entire Brillouin zone using neutron [183], helium atom [184], and thermal diffuse scattering [185].

Figure 1.28 illustrates the softening of the longitudinal phonon mode in the NC phase at room temperature, indicating a Kohn anomaly in the system. While $1 T-\mathrm{TaS}_{2}$ certainly represents a model system to study the effects of CDW formation on the electronic structure of materials, several essential properties and phenomena are still subject to current experimental and theoretical studies. In this context, time-resolved measurements of the electronic and lattice structure have contributed significantly to the understanding of CDWs as will be discussed hereafter. 


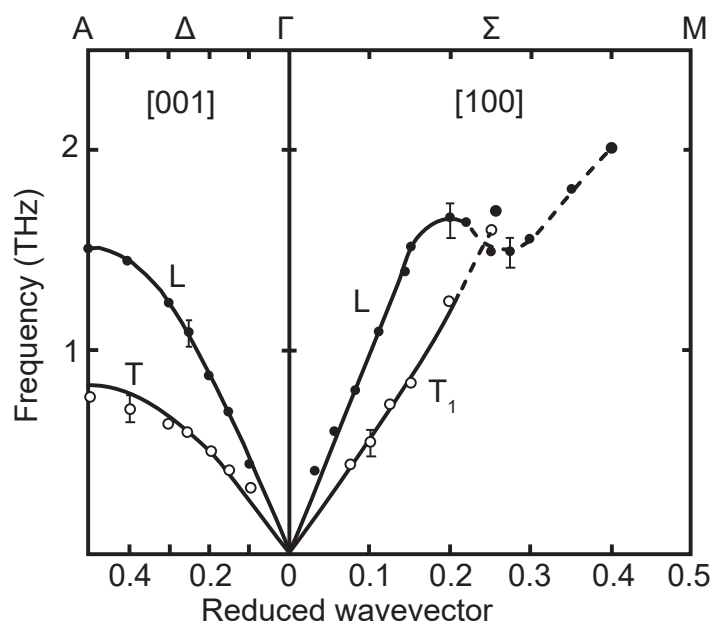

Figure 1.28: Phonon dispersion of $1 T-\mathrm{TaS}_{2}$ at room temperature. The filled and open circles refer to phonons measured in longitudinal and transverse geometry, respectively. Phonon softening emerges between the $\Gamma$ and M-point [183]. (C) IOP Publishing. Reproduced with permission. All rights reserved.

\subsubsection{Previous time-resolved Experiments}

Many time-resolved studies have been performed on $1 T-\mathrm{TaS}_{2}$, investigating the dynamics of singleparticle and collective excitations $[24,34,76,163,173,186-188]$ as well as CDW phase transitions $[7,14,173,189-191]$. A selection of these works will be briefly discussed in this section.
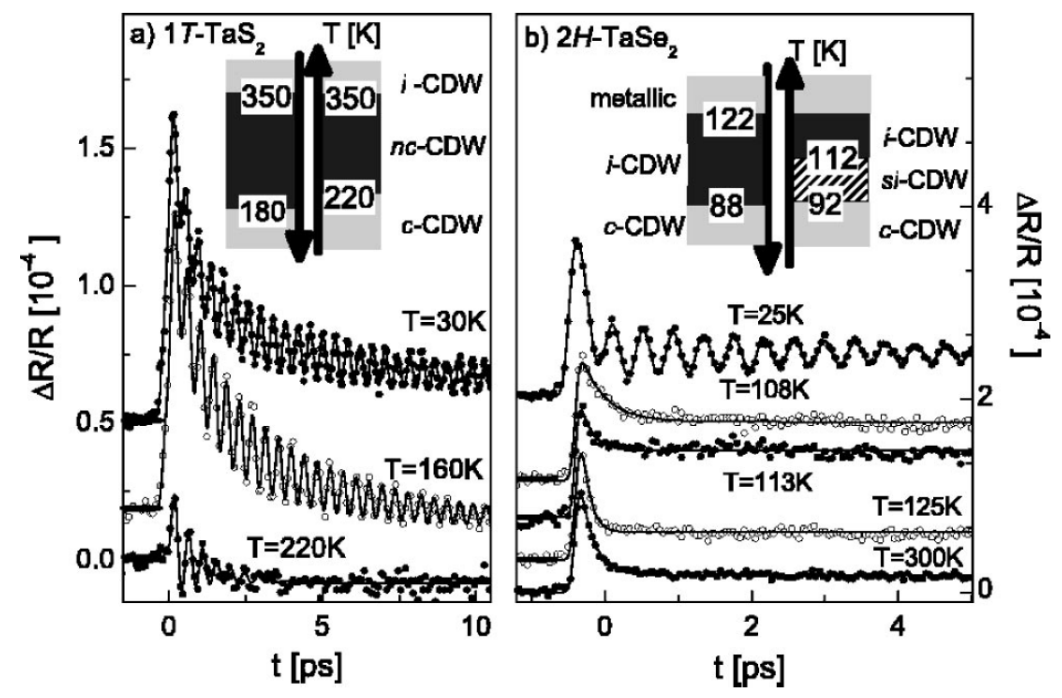

Figure 1.29: Transient reflectivity measurements for $1 T-\mathrm{TaS}_{2}$ (a) and $2 \mathrm{H}-\mathrm{TaSe}_{2}$ (b) at different temperature above and below $T_{\mathrm{C}-\mathrm{NC}}$ and $T_{\mathrm{I}-\mathrm{N}}$, respectively. The signals are offset for clarity. The insets represent the phase diagrams of the corresponding bulk material. Reprinted figure with permission from Ref. [173] by Demsar et al.. Copyright 2002 by the American Physical Society. 
In the work of Demsar et al. [173], the collective and single-particle excitations of $1 T-\mathrm{TaS}_{2}$ (and $2 \mathrm{H}-\mathrm{TaSe}_{2}$ ) were probed by time-resolved optical spectroscopy. This technique measures the transient reflectivity of the material and allows to trace the evolution of above-gap carriers after pulsed optical excitation. For temperatures ranging from $30 \mathrm{~K}$ up to $220 \mathrm{~K}$, the transient reflection $\Delta R / R$ exhibits typical features comprising a strong increase within few femtoseconds followed by a relaxation on a few-picosecond time scale and THz-oscillations around the average value. From these curves, characteristic time constants, oscillation frequencies and the gap size are obtained. The prominent oscillation observed in these measurements is associated with the CDW amplitude mode of the system whose damping is strongly dependent on the temperature. Specifically, for the highest temperature of $220 \mathrm{~K}$, the amplitude oscillation is attenuated after 2-4 ps only (see Fig. 1.29).

Hellmann et al. [163] investigated the C-NC phase transition by means of femtosecond timeresolved core-level photoemission spectroscopy, which allows for measuring the atomic-site-specific charge-order dynamics of the charge-density wave in the low-temperature commensurate phase. More specifically, the $4 \mathrm{f}$ core-level splitting of Ta is a direct measure for the CDW gap $\Delta_{\mathrm{CDW}}$. Upon intense fs-laser illumination, the response consists of a subpicosecond reduction of the CDW-induced splitting and a partial recovery on a few-picosecond time scale into a quasiequilibrium state having a lifetime of more than $10 \mathrm{ps}$. The authors conclude that the two-step melting process is governed by, firstly, a quasi-instantaneous collapse of the charge order due to hot electrons and, secondly, melting of the long-range order of the $\mathrm{C}$ phase via energy transfer to the lattice. Moreover, they point out that the strong coupling of the charge density modulation and the periodic lattice distortion in the equilibrium state is suspended after photoexcitation for the time scale of electron-phonon thermalization.

In a seminal work, Eichberger et al. [24] studied the structural changes of the NC phase using femtosecond electron diffraction experiments in transmission geometry with a temporal resolution of about $250 \mathrm{fs}$, and optical spectroscopy (see Fig. 1.30). They monitored the transient change of main and satellite diffraction reflexes, observing a rapid suppression of the periodic lattice distortion by about $20 \%$ on a timescale of about $250 \mathrm{fs}$ and a subsequent recovery to a thermalized state in about 4 ps (see Fig. 1.30). In particular, while the satellite peak intensity strongly decreases, the main lattice reflex shows a prominent local maximum as the CDW amplitude is quenched. The authors interpret their results based on a common free-energy picture of broken-symmetry states that includes the rapid breakdown of the electronic modulation, the excitation of collective atomic motion during relaxation and the transition to a thermalized state.

In a comparative study, time-resolved ARPES enabled Hellmann et al. [34] to trace the full electronic band dispersion of $1 T-\mathrm{TaS}_{2}, 1 T-\mathrm{TiSe}_{2}$ and intercalated $\mathrm{Rb}: 1 T-\mathrm{TaS}_{2}$ (see Fig. 1.31). 

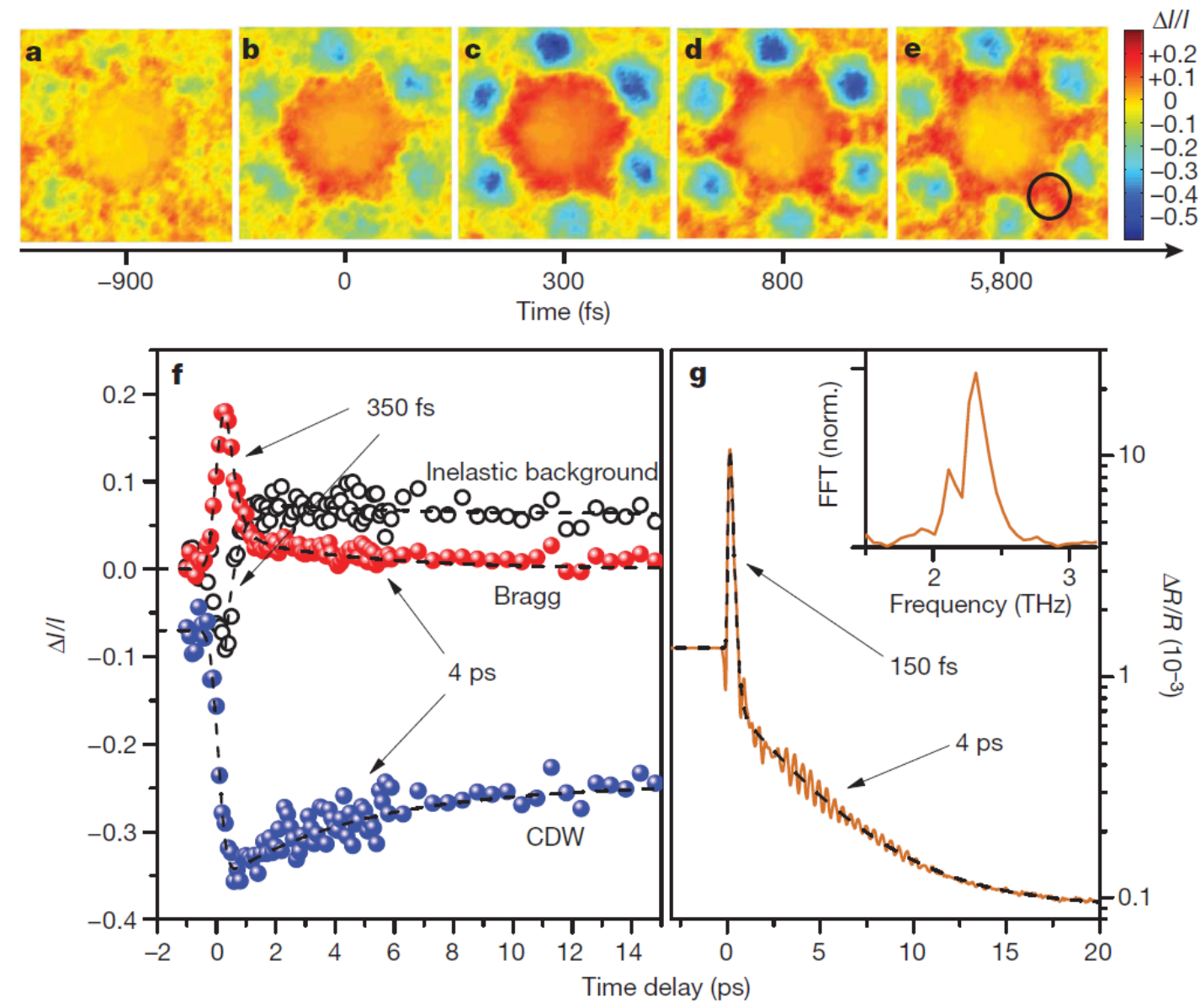

Figure 1.30: Time-dependent diffraction intensities after optical excitation of $1 T-\mathrm{TaS}_{2}$. (a-e) Snapshots of diffraction pattern segment showing main and satellite reflexes as well as the diffuse background for several time delays. (f) Temporal evolution of relative intensities. (g) Transient reflectivity change exhibiting distinct oscillation frequencies (inset). Reprinted by permission from Springer Nature Customer Service Centre GmbH [24], Copyright 2010.

Contrasting the length and excitation-density dependence of the gap melting times, the authors gain detailed insight into the interaction-dependent processes and provide a more reliable classification of the three insulators. The time-dependent results for the $\mathrm{C}$ phase of pristine $1 T-\mathrm{TaS}_{2}$ show the fast Mott and Peierls gap collapse within $<50$ fs and $~ 200$ fs, respectively, and an oscillatory component during relaxation that is associated with the coherent amplitude-mode oscillations of the CDW (see Fig. 1.31), as previously measured by optical spectroscopy.

The selection of works presented gives a brief overview of the transient response of the material, including the electronic and structural degrees of freedom. The associated time scales and excited modes will serve as a reference for the investigation of the IC and NC phase using ULEED in Chapter 3. Special emphasis lies on the relaxation dynamics which exhibit a long-lived structural 
non-equilibrium scenario. While several other recent works studied the ultrafast structural dynamics of $1 T-\mathrm{TaS}_{2}$ [191, 192] and related CDW materials [38, 193-195] using high-energy electrons, ULEED provides a new complementary view on the structural dynamics at the surface.
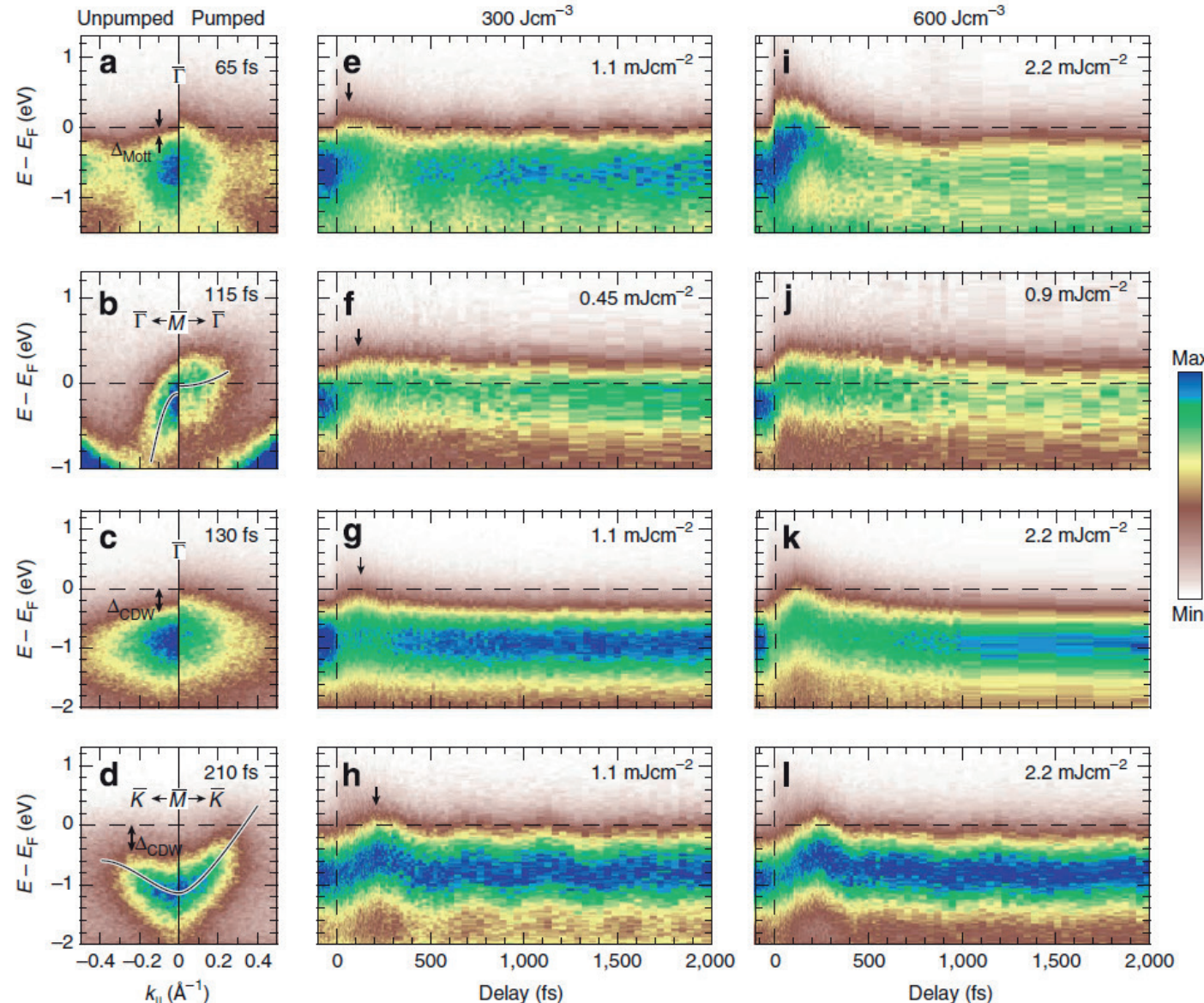

Figure 1.31: Time-resolved electronic band structure for the layered charge-density wave compounds $1 T$ $\mathrm{TaS}_{2}(\mathrm{C}$ phase at $110 \mathrm{~K})\left(\mathrm{a}, \mathrm{e}, \mathrm{j}\right.$ and d,h,l), $1 T-\mathrm{TiSe}_{2}(\mathrm{~b}, \mathrm{f}, \mathrm{j})$ and intercalated $\mathrm{Rb}: 1 T-\mathrm{TaS}_{2}(\mathrm{c}, \mathrm{g}, \mathrm{k})$. While the first column compares unpumped and pumped ARPES spectra, the second and third column show the temporal evolution of momentum-integrated spectra for two different fluences. Reprinted by permission from Springer Nature Customer Service Centre GmbH [34], Copyright 2012. 


\title{
2 | Nanotip-based photoelectron Microgun for ultrafast LEED
}

\author{
G. Storeck, S. Vogelgesang, M. Sivis, S. Schäfer, and C. Ropers
}

Structural Dynamics 4, 044024, April 2017

DOI: $10.1063 / 1.4982947$

We present the design and fabrication of a micrometer-scale electron gun for the implementation of ultrafast low-energy electron diffraction from surfaces. A multi-step process involving photolithography and focused-ion-beam nanostructuring is used to assemble and electrically contact the photoelectron gun, which consists of a nanotip photocathode in a Schottky geometry and an einzel lens for beam collimation. We characterize the low-energy electron pulses by a transient electric field effect and achieve pulse durations of $1.3 \mathrm{ps}$ at an electron energy of $80 \mathrm{eV}$. First diffraction images in a backscattering geometry (at $50 \mathrm{eV}$ electron energy) are shown.

\subsection{Introduction}

Ultrafast electron diffraction [196] and microscopy [2] are rapidly evolving tools for the study of structural dynamics. In recent years, ultrafast variants of numerous techniques employing electrons as structural and spectroscopic probes were developed, including high-energy electron diffraction [197, 198], transmission electron microscopy, [5, 199-204] and electron energy loss spectroscopy $[205,206]$.

One of the particular benefits of electron beams is the high scattering cross-section facilitating surface-sensitive electron diffraction, for example, in reflection high-energy and low-energy electron diffraction (RHEED and LEED). Ultrafast RHEED was implemented early on in Refs. [207] and [208], and its temporal resolution has reached the few-picosecond to femtosecond domain in the 
past few years [23, 209]. However, because of its grazing incidence geometry, the real strength of RHEED is its in-situ capability to characterize growth during epitaxy, rather than to obtain direct representations of the surface structure and symmetry. Some drawbacks of RHEED are enhanced volume contributions for stepped and imperfect surfaces and its restriction to map a limited angular fraction of reciprocal space. Ultrafast low-energy electron diffraction (ULEED), on the other hand, is highly desirable due to LEED's outstanding ability to map atomic-scale surface structures [125] but has remained particularly challenging experimentally [210, 211]. A main obstacle in the implementation of ULEED lies in achieving ultrashort electron probe pulses at low energies, which are extremely susceptible to pulse spreading in the propagation from the electron source to the sample [210, 212]. Recently, employing nanoscale photocathodes [128, 213-218] and minimized propagation distances, this limitation was overcome in a compact transmission ULEED setup for the study of structural dynamics in monolayers and ultrathin films [46]. In a related approach, ultrafast point-projection microscopy was developed [219-221] and applied in the imaging of charge dynamics [219]. Extending the ULEED methodology to a backscattering geometry would enable investigations of ultrafast structural processes at surfaces, but, in order to avoid shadowing of the backscattered diffraction pattern, this requires the development of miniaturized photoelectron sources of sufficiently small outer diameters.

Here, we present the implementation of a nanofabricated electron gun (hereafter referred to as the 'microgun') facilitating ULEED. The microgun consists of a tungsten nanotip photoemitter embedded in a shielded micrometer-scale electrostatic lens assembly (total outer diameter of 80 $\mu \mathrm{m}$; Fig. 2.1d). Utilizing this photoelectron source, we achieve a temporal resolution in electron projection imaging of $1.3 \mathrm{ps}$ at an electron energy of only $80 \mathrm{eV}$ and a source-sample distance of $400 \mu \mathrm{m}$. High-quality electron diffraction patterns are recorded in a backscattering geometry, demonstrating the high spatial coherence of the generated electron beam. This photoelectron gun combines ultrafast temporal resolution with high momentum resolution and ultimate surface sensitivity, promoting access to numerous ultrafast phenomena in the structural dynamics at surfaces.

\subsection{Gun fabrication}

The electrostatic microgun is assembled at the edge of a glass slide, onto which metallic lines (see Figs. 2.1 a and 2.1b) are deposited to connect the micrometer-sized gun electrodes to millimeter-scale pads and the voltage supplies. The chromium contact lines are fabricated using a photolithographic process and subsequently covered with an insulating polyimide layer (Kapton). The polymer and the backside of the glass slide are coated with thermally evaporated titanium films to shield electrical 
stray fields, except for the external contact pads and the strip lines leading to the electron gun (Fig. 2.1b).

The electrode structures comprising the microgun assembly are fabricated by slicing a gold wire using focused-ion-beam etching and are fixed to the edge of a glass slide by ion-beaminduced platinum deposition. In order to minimize the electron propagation distance to the sample, the electrode assembly is placed close to the corner of the contact support. Figure 2.1c displays a scanning electron micrograph of the resulting electron gun with five separate gold electrodes, representing (left to right) the ground, gun lens, extractor, suppressor, and cathode electrodes. The cathode electrode holds a nanometric tungsten tip (focus-ion-beam-prepared, $20 \mu \mathrm{m}$ tip length, radius of curvature below $50 \mathrm{~nm}$, see Figs. 2.1e and 2.1f). Bright areas in the electron micrograph in Fig. 2.1c are due to electron beam induced charging, indicating sufficient insulation between the contact lines (darker regions). At this stage, the outer diameter of the electron gun is approximately $30 \mu \mathrm{m}$. Finally, to minimize electric stray fields, the remaining exposed contact lines and the gun electrodes are shielded by a grounded metal-coated mica plate and a Kapton cylinder, respectively, leading to an effective gun diameter of $80 \mu \mathrm{m}$ (Fig. 2.1d). The aperture in the shielding hull (indicated with the square in Fig. 2.1d) allows for inducing photoemission from the nanometric tungsten tip by side illumination with laser pulses. The magnified view in Fig. 2.1e shows the tip with its apex located in the center between the suppressor and extractor electrodes (approximately 6 $\mu \mathrm{m}$ from the suppressor electrode).

\subsection{Numerical simulations}

In order to estimate the gun performance in terms of temporal resolution and spatial beam parameters, we carried out finite element simulations solving for the electric field and the propagation of electrons in our gun geometry (Fig. 2.2). Generally, the microgun is composed of a source region including a tip, an extractor and a suppressor electrode, an einzel lens formed by the extractor, a gun lens electrode, and a grounded exit aperture (Fig. 2.2a). Electron trajectories are simulated for a range of voltage settings and initial conditions of the electrons emitted from the hemispherical nanotip apex (green lines in Figs. 2.2a and 2.2b). Assuming one to a few electrons per pulse, we do not consider Coulomb interactions between electrons. For each electron kinetic energy (tip bias plus photoemission excess energy), the suppressor and lens electrode voltages are chosen to form a collimated beam exiting the gun, holding the extractor at ground potential (Fig. 2.2c). Trajectories are obtained for a range of emission positions along the apex $\left(0^{\circ}-90^{\circ}\right.$ from the axis), emission angles $\left( \pm 90^{\circ}\right.$ from the surface), and initial kinetic energies $(0-3 \mathrm{eV})$. A total number of about 50.000 

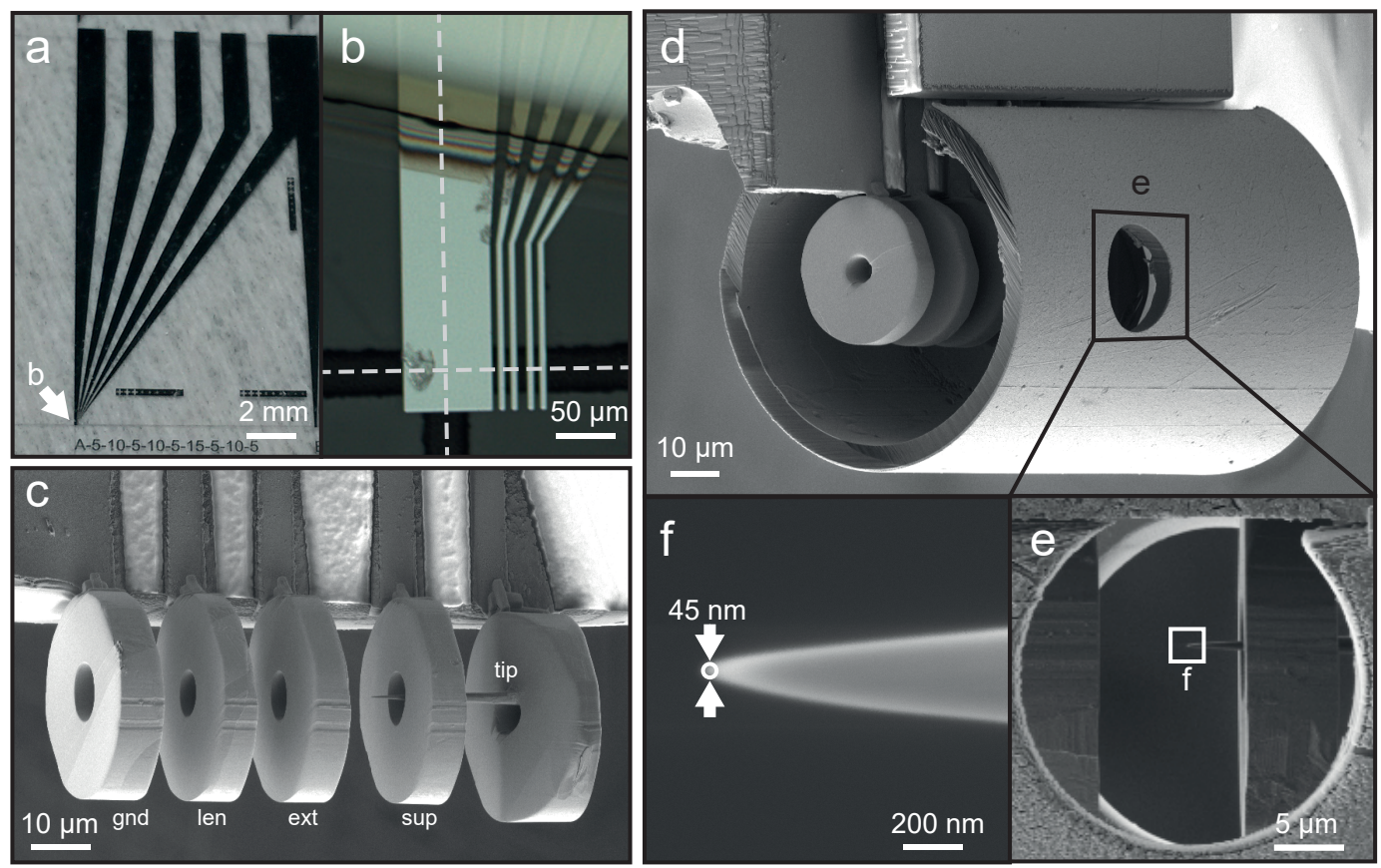

Figure 2.1: (a), (b) Optical microscope images of photolithographically patterned metallic tracks ( $\mathrm{Cr}$ on a $70-\mu \mathrm{m}$ thick Borosilicate glass slide). The electrostatic gun assembly is placed at an edge of the cleaved substrate (dashed lines indicated in (b)). (c)-(f) Scanning electron micrographs of the miniaturized electron gun at various fabrication stages and magnifications. (c) Contacted electrodes of the microgun (without shielding), exhibiting tip, suppressor, extractor, lens, and ground electrodes. (d) Finished microgun with electrostatic shielding attached. (e) Side-view through the laser excitation aperture. (f) Tungsten tip-emitter with a radius of curvature below $50 \mathrm{~nm}$ (prepared by focused-ionbeam etching).

particle trajectories are computed for each bias voltage.

From these trajectories, we predict electron pulse durations by weighting the different trajectories with distributions of the initial kinetic energy and emission angle and position, using procedures outlined in Refs. [212] and [128]. The initial energy distribution is taken as the positive energy half of a Gaussian centered at an energy of $0 \mathrm{eV}$, with a standard deviation (of the corresponding full Gaussian) of $\sigma_{E}$. For different initial kinetic energy widths, Fig. 2.2d displays the resulting electron pulse duration (full-width-at-half maximum, FWHM) in the energy range of $40-100 \mathrm{eV}$, derived from the distribution of arrival times at a plane $400 \mu \mathrm{m}$ behind the exit aperture of the gun. In particular, pulse widths below 1 ps are predicted throughout the energy range shown for initial energy widths of $\sigma_{E} \leq 0.5 \mathrm{eV}$ and at energies $>70 \mathrm{eV}$ for $\sigma_{E} \leq 1 \mathrm{eV}$. Such energy widths were previously observed for two-photon photoemission from tungsten nanotips in Ultrafast Transmission Electron Microscopy (UTEM) [203]. The set of curves in Fig. 2.2d illustrates that both electron 

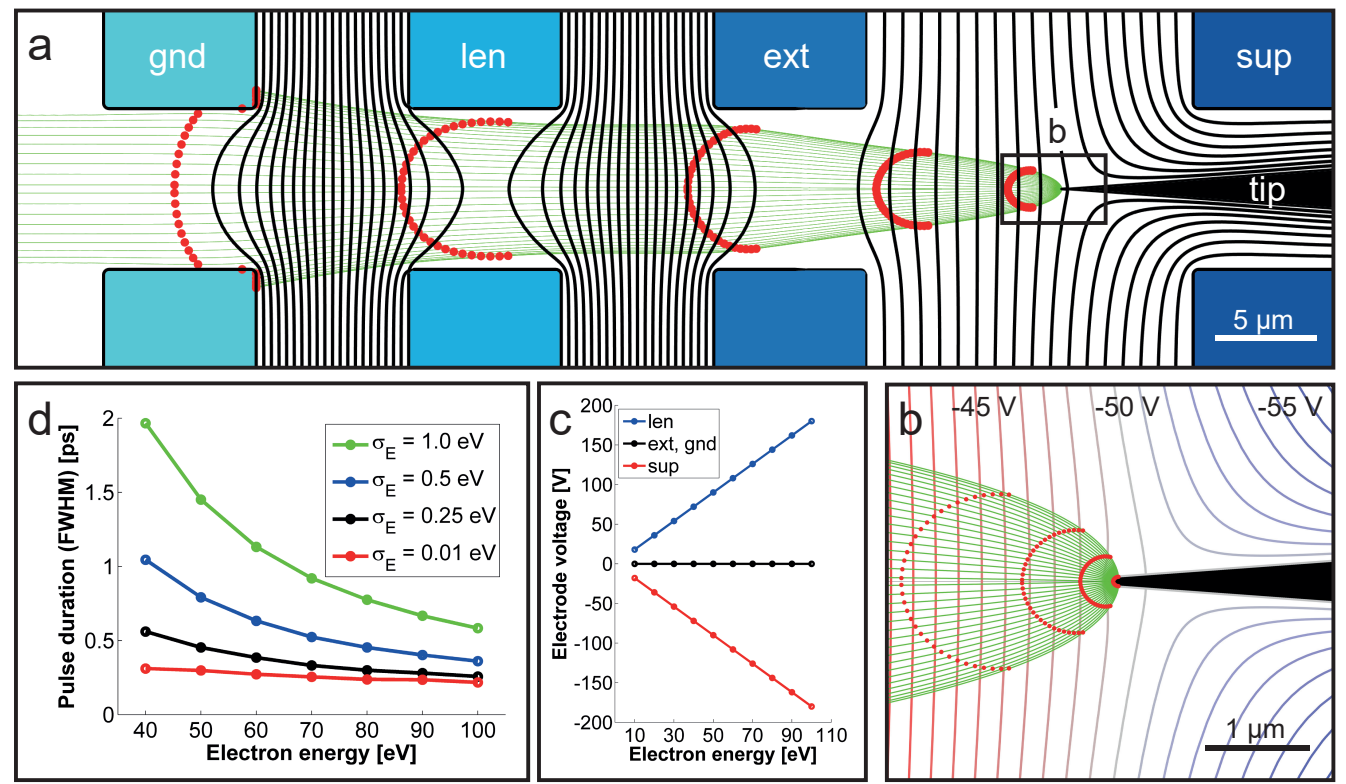

Figure 2.2: (a) Finite element modeling (FEM) of the miniaturized electron gun for a set of applied electrode voltages described by (tip, sup, ext, len, gnd) $=(-50,-90,0,90,0) \mathrm{V}$. Cylindrical symmetry is assumed. Solid black: equipotential lines, green: electron trajectories. (b) Magnified view of the tip region. (c) Energy-dependent voltage setting applied to electrodes for a collimated electron beam. (d) Pulse duration versus electron energy at a plane $400 \mu \mathrm{m}$ behind the ground electrode, for various widths in the initial kinetic energy distribution. Red dots in (a) and (b) indicate the positions of groups of electrons emitted at the same time.

velocity dispersion and path length differences contribute to the final electron pulse duration. For the present design, path length differences amount to a pulse spreading of about 200-300 fs across the energy range plotted (red line, quasimonochromatic initial energy distribution), which could be further reduced by the application of a higher extraction field or a smaller exit aperture.

\subsection{Experimental Results}

In the following, we experimentally characterize the pulse duration and the beam quality of the ultrafast photoelectron microgun. To this end, the gun is mounted inside an ultrahighvacuum (UHV) chamber (base pressure $7 \times 10^{-10} \mathrm{mbar}$ ) and connected to computer-controlled voltage supplies. Two-photon photoelectron emission (identified by a quadratic intensity scaling of the photoemission current) is induced by focusing 400-nm femtosecond laser pulses (duration $80 \mathrm{fs}$, repetition rate $312 \mathrm{kHz}$, and pulse energy $110 \mathrm{pJ}$ ) onto the tungsten nanotip emitter using a plano-convex lens on a motorized linear 3D stage (focal length $23 \mathrm{~mm}$ focus diameter approx. $5 \mu \mathrm{m}$ ), resulting in the 
emission of about one electron per pulse [46, 128, 204].

The electron pulse duration is measured by using a previously established method based on transient electric fields [218, 222, 223]. Specifically, the photoelectron beam is directed through a transmission electron microscope (TEM) copper mesh covered with a finer amorphous carbon grid (Figs. 2.3a-2.3c). For the lens potential at ground, a divergent beam is produced that results in a projection image of the TEM grid (Figs. 2.3a and 2.3d) with a magnification of about 200, which is recorded using a phosphor-screen microchannel plate $(5 \mathrm{~cm}$ behind sample) and a CCD camera. In the pulse duration measurement, the projection image is distorted by a spacecharge cloud near the sample, induced by an intense pump laser pulse (duration $80 \mathrm{fs}$, center wavelength $800 \mathrm{~nm}$, and fluence up to $2 \mathrm{~mJ} / \mathrm{cm}^{2}$ ), and the images are taken for variable optical-pump/electron-probe delays (see Figs. 2.3a and 2.3d). A delay-dependent series of projections (Fig. 2.3d) and difference images with respect to a fixed negative time delay (Fig. 2.3e) show a pump-induced contrast change over time. Evaluating the delay-dependent image contrast, we observe dynamical features as rapid as 1.3 ps (Fig. 2.3f), which represents an upper bound to the local electron pulse duration.
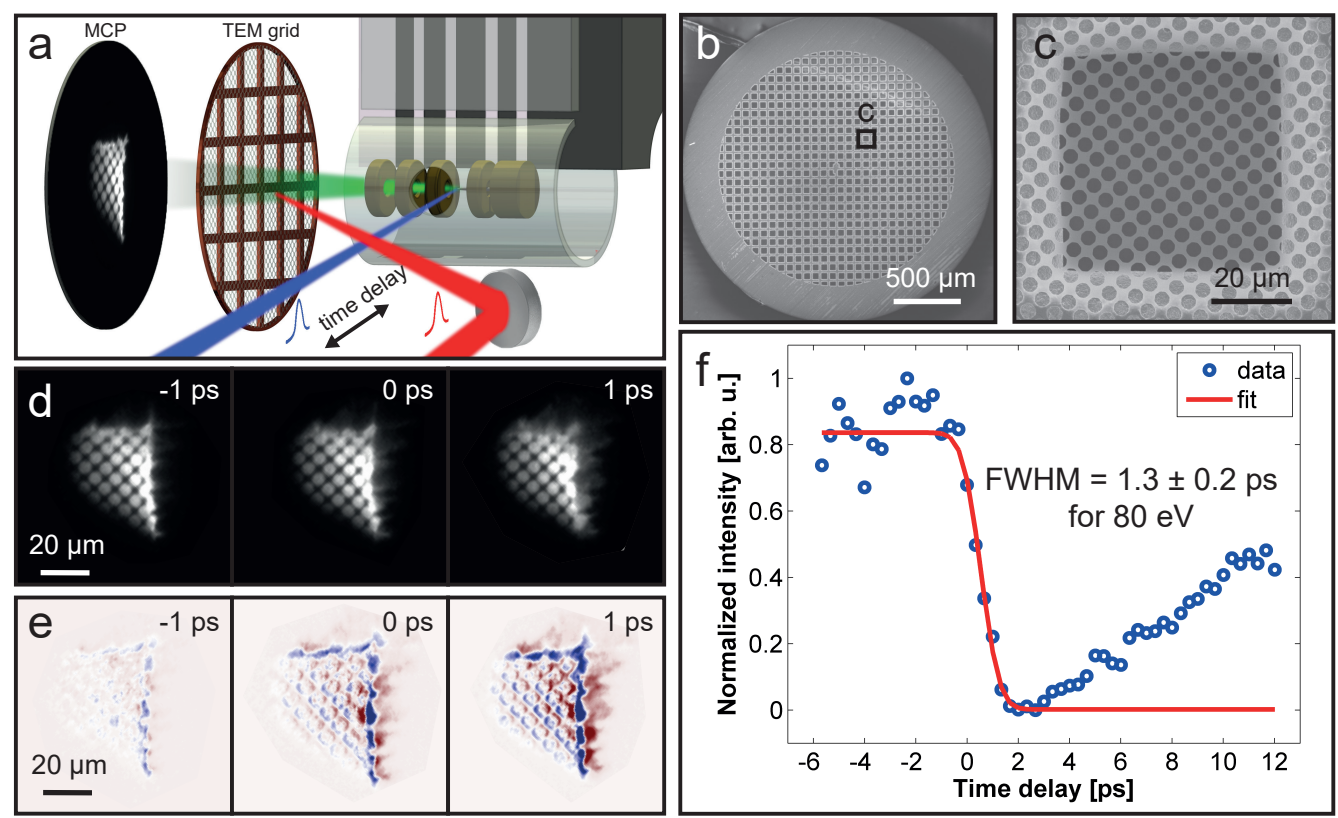

Figure 2.3: (a) Schematic of the experimental setup for characterizing electron-pulse durations via the transient electric field effect (not to scale). (b) and (c) TEM copper grid (square opening with $50 \mu \mathrm{m}$ width), covered with a perforated carbon film (Quantifoil, circular apertures of $3.5 \mu \mathrm{m}$ diameter). (d) Projection images recorded with photoelectrons from the microgun for different pump-probe time delays. (e) Difference in images taken at the given delay and a large negative delay $(<-20 \mathrm{ps})$. (f) Contrast change fitted with an error function (red line), resulting in a temporal resolution of 1.3 ps or shorter at an electron energy of $80 \mathrm{eV}$. 
In Fig. 2.4, we demonstrate the operation of the microgun in backscattering diffraction-mode. LEED images from a single-crystalline surface of the transition metal dichalcogenide $1 T-\mathrm{TaS}_{2}$ (cleaved in UHV) are recorded, as shown in Fig. 2.4b. This material exhibits a periodic lattice distortion (PLD) associated with a charge-density wave (CDW) [159], which results in a complex pattern of superstructure diffraction spots. Atomic lattice Bragg spots (Fig. 2.4b, red circles) and PLD spots of different orders (all other peaks) are clearly resolved. For approximate diffraction probabilities of $1 \%-3 \%$ and one incident electron per pulse, $10^{5}-10^{6}$ electrons are detected in images within one to few minutes of exposure (Figs. 2.4b and 2.4c). In order to map a large number of Bragg conditions, the backscattered electron diffraction pattern was recorded with a gun-sample distance of $550 \mu \mathrm{m}$. This gun-sample distance was determined using a series of diffraction patterns at different positions in front of the sample, using the linear scaling of the respective shadow diameters with the change in the working distance. Restricting the pattern to smaller parts of reciprocal space allows us to reduce the sample-gun distance to $270 \mu \mathrm{m}$ (Fig. 2.4c) or below, so that electron pulse durations as in the projection geometry (Fig. 2.3) are expected. From the minimum peak width observed $\left(0.025 \AA^{-1}\right)$, we determine a transfer width of $25 \mathrm{~nm}$. Combined with a beam diameter on the sample of approximately $3 \mu \mathrm{m}$, we estimate a normalized beam emittance of $200 \mathrm{~nm}$ mrad. This emittance compares favorably with most commercial LEED instruments and is largely caused by the small electron beam source size. A further reduction of the emittance may be achieved by reducing the exit aperture diameter.
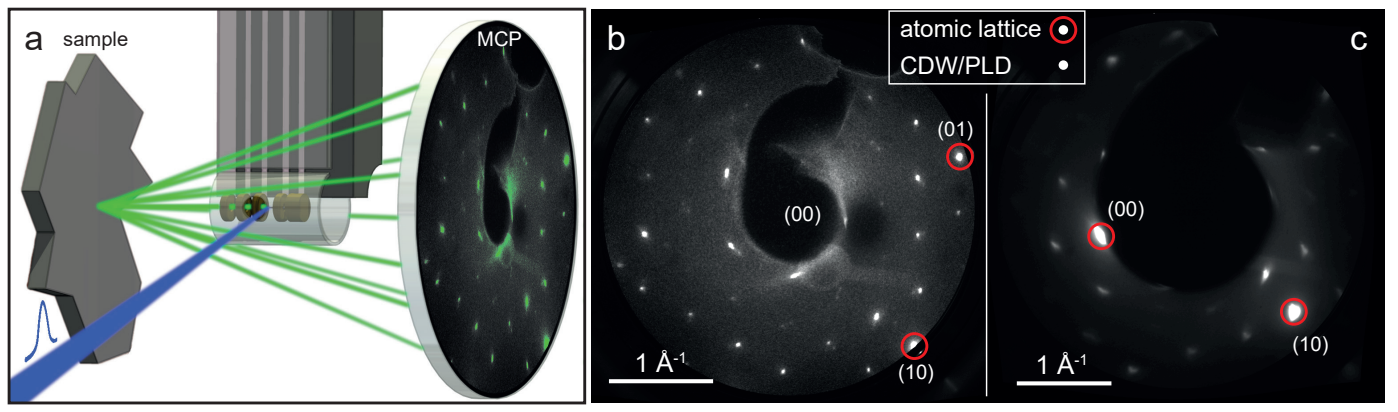

Figure 2.4: (a) Schematic of backscattering diffraction using the microgun (not to scale). Sample-detector distance: $55 \mathrm{~mm}$. The maximum diffraction angle is approximately $35^{\circ}$. (b)-(c) LEED patterns of $1 T-\mathrm{TaS}_{2}$ for an electron energy of $50 \mathrm{eV}$, corrected for distortions caused by the planar detector. Gun-sample distance: $550 \mu \mathrm{m}$ (b) and $270 \mu \mathrm{m}$ (c). Integration time: $100 \mathrm{~s}$ (b) and $600 \mathrm{~s}$ (c). 


\subsection{Conclusion}

We described the fabrication and characterization of a micrometer-scale ultrafast photoelectron gun using photolithography and focused-ion-beam processing. Electron pulse widths of to $1.3 \mathrm{ps}$ at $80 \mathrm{eV}$ were observed, in agreement with numerical simulations for this gun geometry. Even shorter pulse durations could be achieved by further reducing the gun-sample distance, higher extraction fields at the tip apex, or by minimizing the photoemission excess energy using a lower photon energy. At present, the overall size of the gun assembly is limited by the breakdown voltages of the metallic lines and the thickness of the supporting substrate. Besides its benefits in the temporal resolution, the nanolocalized photoelectron source employed has demonstrated its potential to yield high-resolution LEED images. In the future, this microgun and its further developments will promote ultrafast LEED studies with picosecond and femtosecond temporal resolutions, providing direct access to structural dynamics at surfaces and surface reconstructions or in molecular adsorbate layers.

\subsection{Acknowledgments}

We would like to thank Kerstin Hanff and Kai Rossnagel for providing us single-crystalline $1 T-\mathrm{TaS}_{2}$ samples. This work was funded by the European Research Council (ERC-StG 'ULEED'). 


\section{3 | Structural Dynamics of incommen- surate Charge-Density Waves tracked by Ultrafast Low-Energy Electron Diffraction}

G. Storeck, J. G. Horstmann, T. Diekmann, S. Vogelgesang, G. von Witte, S. V. Yalunin, K.Rossnagel, and C. Ropers

Structural Dynamics 7, 044024, June 2020

DOI: $10.1063 / 4.0000018$

We study the non-equilibrium structural dynamics of the incommensurate and nearly commensurate charge-density wave phases in $1 T-\mathrm{TaS}_{2}$. Employing ultrafast low-energy electron diffraction (ULEED) with 1 ps temporal resolution, we investigate the ultrafast quench and recovery of the CDW-coupled periodic lattice distortion. Sequential structural relaxation processes are observed by tracking the intensities of main lattice as well as satellite diffraction peaks and the diffuse scattering background. Comparing distinct groups of diffraction peaks, we disentangle the ultrafast quench of the PLD amplitude from phonon-related reductions of the diffraction intensity. Fluence-dependent relaxation cycles reveal a long-lived partial suppression of the order parameter for up to 60 picoseconds, far outlasting the initial amplitude recovery and electron-phonon scattering times. This delayed return to a quasi-thermal level is controlled by lattice thermalization and coincides with the population of zone-center acoustic modes, as evidenced by a structured diffuse background. The long-lived non-equilibrium order parameter suppression suggests hot populations of CDW-coupled lattice modes. Finally, a broadening of the superlattice peaks is observed at high fluences, pointing to a nonlinear 
generation of phase fluctuations.

\subsection{Introduction}

The spontaneous breaking of a continuous symmetry is a fundamental concept of physics with broad relevance in such diverse areas as particle physics [85], cosmology [86, 87], and condensed matter physics $[64,88]$. An essential consequence of this symmetry breaking is the emergence of new amplitude and phase excitations of the fields considered, exemplified in the Higgs mechanism [89] and massless Nambu-Goldstone bosons [90, 224], respectively. Moreover, the degenerate ground state of such systems allows for nontrivial topological states, as in the case of magnetic vortices [88].

Electron-lattice interaction is an important source of symmetry breaking in solids, most prominently in superconductivity and the formation of charge-density wave (CDW) phases [26, 32, 33, 225]. Specifically, CDWs constitute a periodic modulation of the charge density by electron-hole pairing [26], coupled to a periodic lattice distortion (PLD) [40, 147, 154] and an electronic gap $[39,178,182,226]$. The emergence, correlations and fluctuations of symmetry-broken CDW states can be revealed in the time domain by ultrafast measurement techniques. In this way, quenches of the electronic gap coupled to coherent amplitude oscillations [37, 76, 163, 173, 187, 188, 227], light-induced PLD dynamics [24, 194, 195, 228] and phase transitions have been investigated [163, $229,230]$. In particular, ultrafast structural probes trace changes of structural symmetry [191, 231] and long-range ordering following a phase transformation [14, 189].

However, while the initial quench and coherent amplitude dynamics of CDW systems following short-pulsed excitation are rather well-characterized [76, 163, 173, 187, 188, 227], the subsequent path to thermal equilibrium, including the roles of different collective modes in re-establishing a thermal CDW state, are far less understood. In particular, a sensitive structural probe is required to study the interplay of CDW-coupled excitations and regular phonons.

Here, we employ ultrafast low-energy electron diffraction, a recently developed surface-sensitive structural probe [14, 46-48], to give a comprehensive account of the non-equilibrium structural dynamics of the incommensurate charge-density wave phases at the surface of $1 T-\mathrm{TaS}_{2}$. Harnessing the sensitivity of ULEED to the out-of-plane periodic lattice displacements of the sulfur atoms, we isolate the dynamics of an optically-induced amplitude quench from a multi-stage excitation of phonons. Following a rapid partial recovery, we observe a surprisingly long-lived non-thermal amplitude suppression that equilibrates only after approximately 60 ps. Energy transfer to acoustic phonons is required to re-establish a thermal value of the PLD amplitude, suggesting that transient populations of collective CDW modes have a lasting impact on the structural order parameter. 


\subsection{Materials System and Experimental Approach}

In this work, we study one of the most prominent CDW systems, $1 T-\mathrm{TaS}_{2}$, which is part of the class of transition metal dichalcogenides. The atomic structure of this material consists of weakly interacting S-Ta-S trilayers $[42,161]$, in which the tantalum atoms are octahedrally coordinated between the sulfur atoms (Fig. 3.1a). This compound has attracted much attention for its various CDW phases [26, 42, 161, 232], excitations [24, 34, 76, 173, 187, 188] (Fig. 3.1c), and correlation effects [233-235], serving as a model system to study, for example, Peierls- versus Mott-type metalinsulator transitions [72, 227], pressure-induced superconductivity in coexistence with CDWs [157], transitions to metastable 'hidden' CDW states [7, 230], the emergence of complex orbital textures [176], or quantum spin liquid behavior [236].

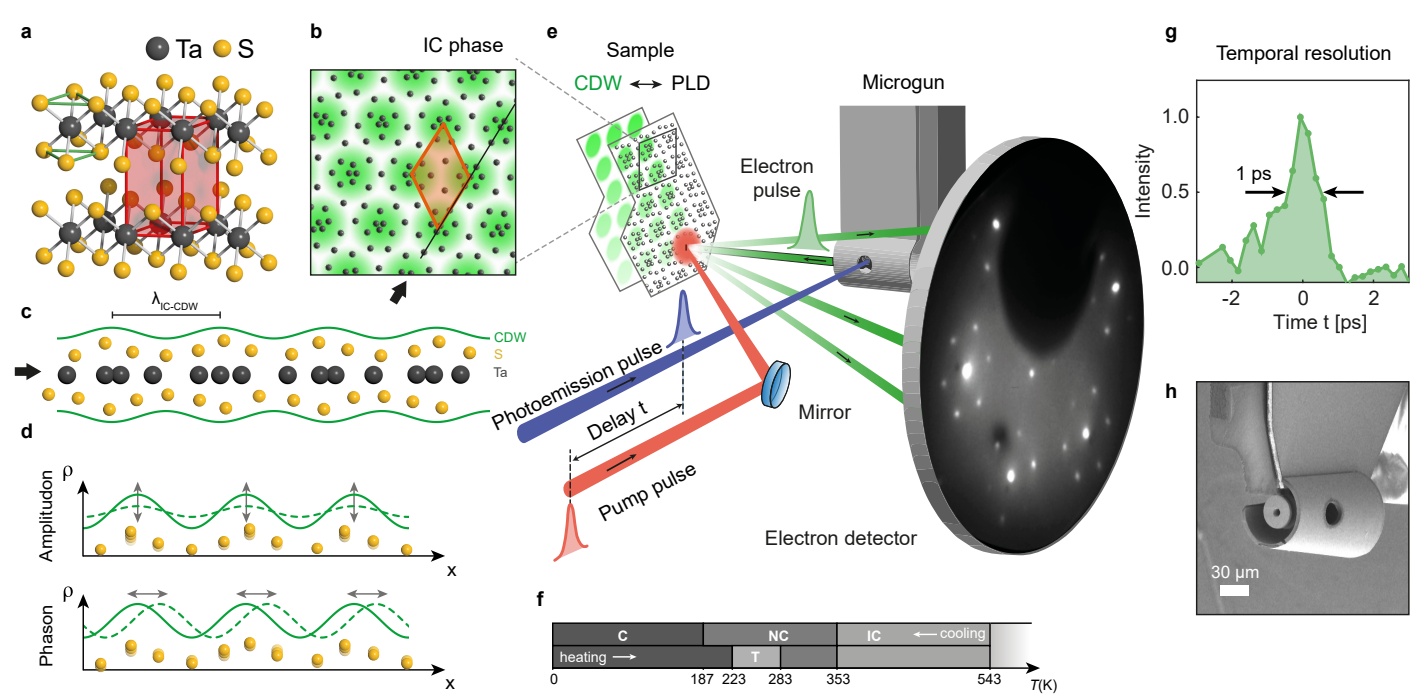

Figure 3.1: Materials system and experimental setup. (a) Layered transition metal dichalcogenide $1 T-\mathrm{TaS}_{2}$ exhibiting a trigonal crystal structure in the high-temperature phase (green lines: octahedral $1 T$-coordination; red: unit cell). (b) Top view of incommensurate (IC) CDW phase illustrating charge density (green), distorted lattice (black dots: Ta atoms, displacements exaggerated) and superstructure unit cell (orange). (c) Side view of a single S-Ta-S trilayer, illustrating the out-of-plane periodic lattice displacements of the sulfur atoms (exaggerated). (d) 1D sketch of CDW amplitude and phase excitations and corresponding lattice fluctuations. (e) Schematic of the experimental setup, showing ultrafast LEED in a backscattering geometry. Ultrashort electron pulses (green) from a nanofabricated electron gun probe the dynamical evolution of the laser-excited surface structure. (f) Temperature-dependent CDW phases. (g) Achieved electron pulse duration of 1 ps (see Appendix 3.6.1 for details). (h) Scanning electron micrograph of miniaturized electron gun.

The material exhibits multiple temperature-dependent phases (Fig. 3.1f) with characteristic lattice deformations coupled to electronic structure changes [42, 72, 237]. Starting from a metallic phase 
with an undistorted trigonal structure (Fig. 3.1a) above $543 \mathrm{~K}$, the system undergoes a sequence of CDW transitions, forming a commensurate (C) (Mott-insulating) state below $187 \mathrm{~K}$. At intermediate temperatures, two incommensurate phases are found, namely the so-called 'nearly commensurate' (NC) phase (187-353 K), exhibiting commensurate patches separated by discommensurations [161, 166, 167, 238], and a homogeneous, fully incommensurate (IC) structure (Fig. 3.1b) between $353 \mathrm{~K}$ and $543 \mathrm{~K}$. The periodic lattice distortions in these phases are characterized by primarily in-plane and out-of-plane displacements of the tantalum and sulfur atoms, respectively (Figs.1b, c). Ultrafast transitions between and manipulation of these phases, as well as their collective modes (Fig. 3.1d) have been observed in various diffraction and spectroscopy studies $[14,16,24,34,38,76,163,173$, 186-188, 190, 191, 227, 239, 240].

In our experiments, we employ pulses of electrons at low energies, typically in the range of 40-150 eV, to probe the structural evolution of the NC and IC states in backscattering diffraction. Ulrafast low-energy electron diffraction $[14,46-48]$ allows us to trace the changes of the diffraction pattern in the time domain, following intense fs-laser illumination (red pulse in Fig. 3.1e). In this optical-pump/electron-probe scheme, excitation and relaxation processes are sampled by varying the time delay $t$ between the optical pump pulse (red) and the photoemission pulse (blue) generating the electron probe (green). Reducing electron pulse broadening by short propagation lengths, a miniaturized electron gun (Fig. 3.1h) [47] allows for a temporal resolution of 1 ps (Fig. 3.1g). Further experimental details are provided in Appendix 3.6.1 (Fig. 3.7).

To facilitate the discussion, we focus the presentation on the response of the IC phase, which has not been studied by ultrafast diffraction, and provide a comprehensive data set of related observations for the NC phase in Appendix 3.6.2.

The IC phase exhibits a triple-Q CDW/PLD, with lattice displacements for each unit-cell atom of the form $[77,78]$

$$
\mathbf{u}(\mathbf{L})=\sum_{i=1,2,3} \mathbf{A}_{i} \sin \left(\mathbf{Q}_{i} \cdot \mathbf{L}+\varphi_{i}\right)
$$

for lattice sites $\mathbf{L}, \mathrm{CDW}$ wavevectors $\mathbf{Q}_{i}$ and phases $\varphi_{i}$. The CDW/PLD texture of a 'dot-lattice' arises for the phasing condition $\sum_{i} \varphi_{i}=0$, and for symmetry reasons, the individual plane wave components share a common amplitude $A=\left|\mathbf{A}_{i}\right|$. The PLD at a wavelength $\lambda_{I C}=3.53 a$ (a: lattice constant) leads to characteristic arrangements of satellite peaks [94, 95] around the main lattice diffraction spots, seen in the ULEED pattern displayed in Fig. 3.2c. As the IC state wave vectors are collinear to the lattice vectors, the satellites are located on the lines connecting the main reflexes. Due to the harmonic (and weak) structural modulation [166, 167], only first-order satellites are observed, with an intensity [94] $I_{\text {sat }} \sim\left|J_{1}\left(\mathbf{s} \cdot \mathbf{A}_{i}\right)\right|^{2} \sim A^{2}$ (s: scattering vector). We note that in 

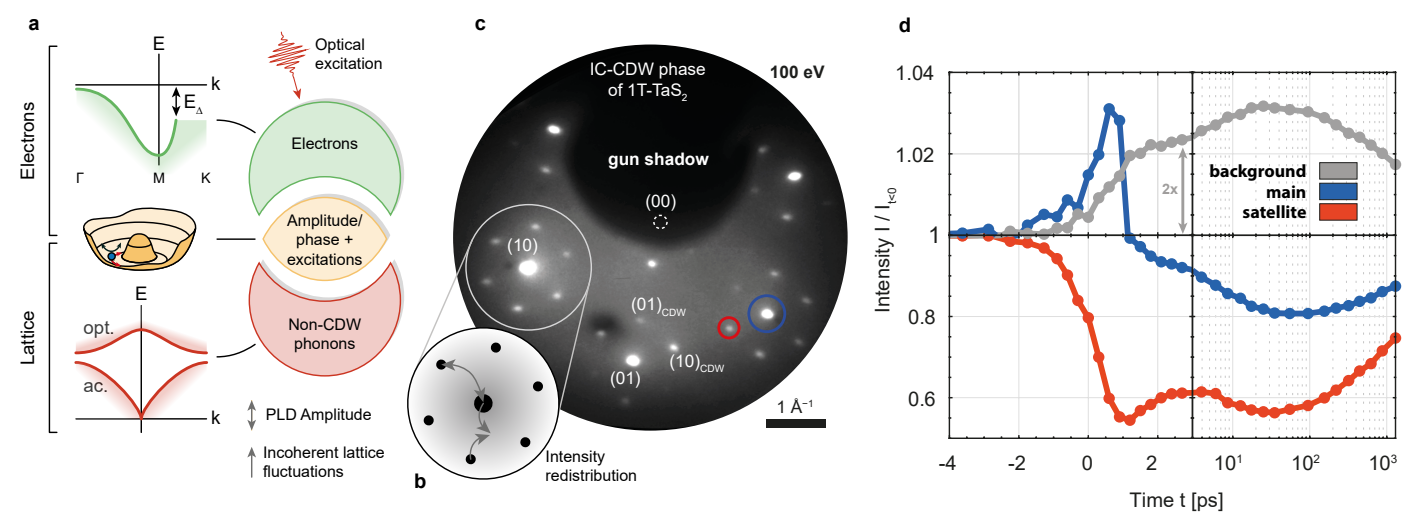

Figure 3.2: Dynamics and excitations in CDW systems influencing diffraction. (a) Electron and lattice subsystems (right) governing CDW dynamics. Gapped band structure (top, left), symmetry broken CDW state with phase and amplitude excitations (middle), and non-CDW phonons (bottom). (b) Changes in average amplitude and all lattice excitations (CDW and non-CDW) lead to a redistribution of intensity in the electron diffraction pattern. (c) Diffraction pattern of the IC phase of $1 T-\mathrm{TaS}_{2}$ showing main lattice reflexes and first-order PLD-induced satellites (integration time: $90 \mathrm{~s}$, electron energy: $100 \mathrm{eV}$ ). (d) Time-dependent measurement of reflexes (blue and red circles in (c)) and diffuse background (fluence $F=2.5 \mathrm{~mJ} / \mathrm{cm}^{2}$ ). The main lattice signal is averaged over (10) and (-1 1) spots (blue), the satellite signal over several reflexes. Curves are normalized to the signal at negative times.

this energy range, LEED is a very efficient structural probe of the PLD, because (i) backscattering diffraction is dominated by the sulfur sublattice, and (ii) the large out-of-plane momentum transfer enhances the sensitivity to out-of-plane displacements.

We study the excitation and relaxation of the IC and NC phases, without driving the system across a phase transition $[14,77,167,189,191,228]$. The dynamics of this incommensurate Peierls system can be discussed based on a simplified picture of three coupled subsystems, namely, the electronic system exhibiting a gapped band structure (Fig. 3.2a, top), the collective amplitude and phase excitations around the symmetry-broken CDW ground state (center) [26], and the ordinary lattice modes far from the CDW wavevector in reciprocal space, i.e., regular phonons (bottom).

It is widely established that electronic excitation by an ultrashort laser pulse induces a carrier population above the band gap, which results in a quench of the CDW/PLD amplitude that recovers upon carrier cooling by electron-phonon scattering [24, 34, 187]. The corresponding sequence of relaxation processes involving the three subsystems causes characteristic changes to the diffraction intensities of the satellite peaks and the main peaks (intensity $I_{\text {main }}$ ). Specifically, for small PLD 
amplitudes, the peak intensities are expected to scale as $[94,95,131,151]$ :

$$
\begin{aligned}
& I_{\mathrm{sat}} \sim A^{2} e^{-2 W_{\varphi}} e^{-2 W_{\mathrm{s}}}, \\
& I_{\text {main }} \sim\left(1-c_{\mathrm{s}} A^{2}\right) e^{-2 W_{\mathrm{s}}},
\end{aligned}
$$

These expressions reflect that a light-induced quench of the mean PLD amplitude $A$ will lead to a redistribution of intensity from the satellites to the main peaks [24, 194, 241]. Different main reflexes are sensitive to the PLD to a varying degree, which requires the introduction of the factor $c_{\mathbf{S}}$ that depends on the momentum transfer $\mathbf{s}$. Inelastic scattering by generated phonons transfers intensity from the reflexes to a diffuse background (Fig. 3.2b) [125, 138, 141, 242], leading to a peak suppression by a Debye-Waller factor $\exp \left(-2 W_{\mathbf{s}}\right)[125,131]$. The general form of the exponent $[125$, 131] $W_{\mathbf{s}} \sim \sum_{p h}\left(\mathbf{s} \cdot \mathbf{u}_{p h}\right)^{2}$ sums over the momentum transfer projected onto phonon displacements $\mathbf{u}_{p h}$ in various branches. According to Overhauser [94], phase fluctuations result in the additional 'phason Debye-Waller factor' $e^{-2 W_{\varphi}}=e^{-\left\langle\varphi^{2}\right\rangle}$, which only affects the satellite spots and also causes diffuse scattering in the vicinity of the satellite peaks [36, 151]. Finally, dislocation-type topological defects in the CDW may broaden the superlattice peaks and also reduce the PLD in the dislocation core $[14,38]$.

\subsection{Results and Analysis}

Our ULEED experiments directly show the characteristic diffraction changes mentioned above: In the exemplary data displayed in Fig. 3.2d, a main lattice peak (blue) exhibits a transient intensity increase after the pump pulse, before experiencing an initially rapid and then slowed suppression to a minimum at $t=60 \mathrm{ps}$. The satellite peaks (red), on the other hand, are first suppressed, before approaching a similar trend as the main peak beyond approximately $10 \mathrm{ps.} \mathrm{Both} \mathrm{the} \mathrm{satellite} \mathrm{and}$ main peak intensities are significantly reduced by phonon populations [125]. These are evident in the diffuse background (gray), which mirrors the suppression of the reflexes, with a step-like increase in the first ps and a slower rise to a maximum at the delay of $60 \mathrm{ps}$. The initial step can be interpreted as the excitation of a broad population of optical and acoustic phonons on the timescale of electron-phonon energy relaxation $(<1 \mathrm{ps})$ [173], while the slower timescale corresponds to phonon-phonon equilibration [243] and the population of low-energy acoustic modes. LEED intensities are rather sensitive to the large amplitudes of low-frequency modes, particularly those with out-of-plane polarization. Specifically, phonon modes with out-of-plane displacements $\mathbf{u}_{p h}$ have a more pronounced Debye-Waller factor due to the backscattering geometry with a primarily out-of-plane scattering vector of the electron. In addition, these modes exhibit comparatively slow 
phase velocities, as is typical for layered van-der-Waals materials [244]. Thus, the prominent main lattice suppression evolving over tens of picoseconds primarily stems from the increasing population of low frequency acoustic modes modulating the layer distance.

These strong Debye-Waller factors complicate an analysis of the temporal evolution of the amplitude quench. On the other hand, our experimental data shows that the different reflexes share a common phonon-induced peak suppression. In the following sections 3.3.1 and 3.3.2, we pursue two approaches of disentangling the dynamics of the structural order parameter from the phonon population, exploiting (see Section 3.3.1) the different sensitivities of two inequivalent classes of main lattice reflections to the PLD, and (see Section 3.3.2) the direct sensitivity of the satellite reflexes to the PLD.

\subsubsection{Amplitude Analysis based on Main Lattice Reflexes}

Concerning the time-dependent peak intensity, the main reflexes fall into two different groups. Whereas all five visible main peaks show a suppression opposite to the increase in diffuse background (Fig. 3.3a), we find that the transient amplitude signal is prominent only in the (1 0$)$ and (-1 1) peaks, while it is largely absent in the $(01),(-10)$, and $(1-1)$ peaks (see also difference maps in Fig. 3.3b) [245]. These two groups of peaks are crystallographically distinct, and the peaks within each group are equivalent in the effective threefold symmetry of the 1T structure [129]. The different sensitivity of the peak intensities to the PLD is a particular feature of LEED, as described in the following.

In the electron energy range of 70-110 eV, diffraction intensities are mainly governed by scattering from sulfur atoms, due to large atomic scattering factors $[129,161]$. As a result of the CDWinduced contraction of the tantalum sub-lattice, the sulfur atoms predominantly exhibit out-ofplane displacements. In backscattering, the opposing directions for the displacements in the upper and lower sulfur layers within each S-Ta-S trilayer $[129,161]$ (Fig. 3.1c) lead to an interference with enhanced or suppressed sensitivity of the two groups of main lattice peaks to the lattice distortion. This feature is expected in all CDW phases of $1 T-\mathrm{TaS}_{2}$, which share the phasing condition mentioned above (compare Fig. 3.1b). Experimentally, we found the same trend in experiments on the NC phase (see Appendix 3.6.2), which exhibits different wavevectors but the same phasing between the three CDWs. In order to further corroborate these findings and considering the importance of multiple scattering in LEED, we conducted dynamical LEED simulations for a PLD of a varying amplitude and as a function of the electron beam energy (see Appendix 3.6.6). In these simulations, for computational reasons, the commensurate modulation was employed, taking quantitative displacements from a recent LEED reconstruction [129]. Importantly, the dynamical LEED simulations qualitatively reproduce our experimental findings of different sensitivities to 

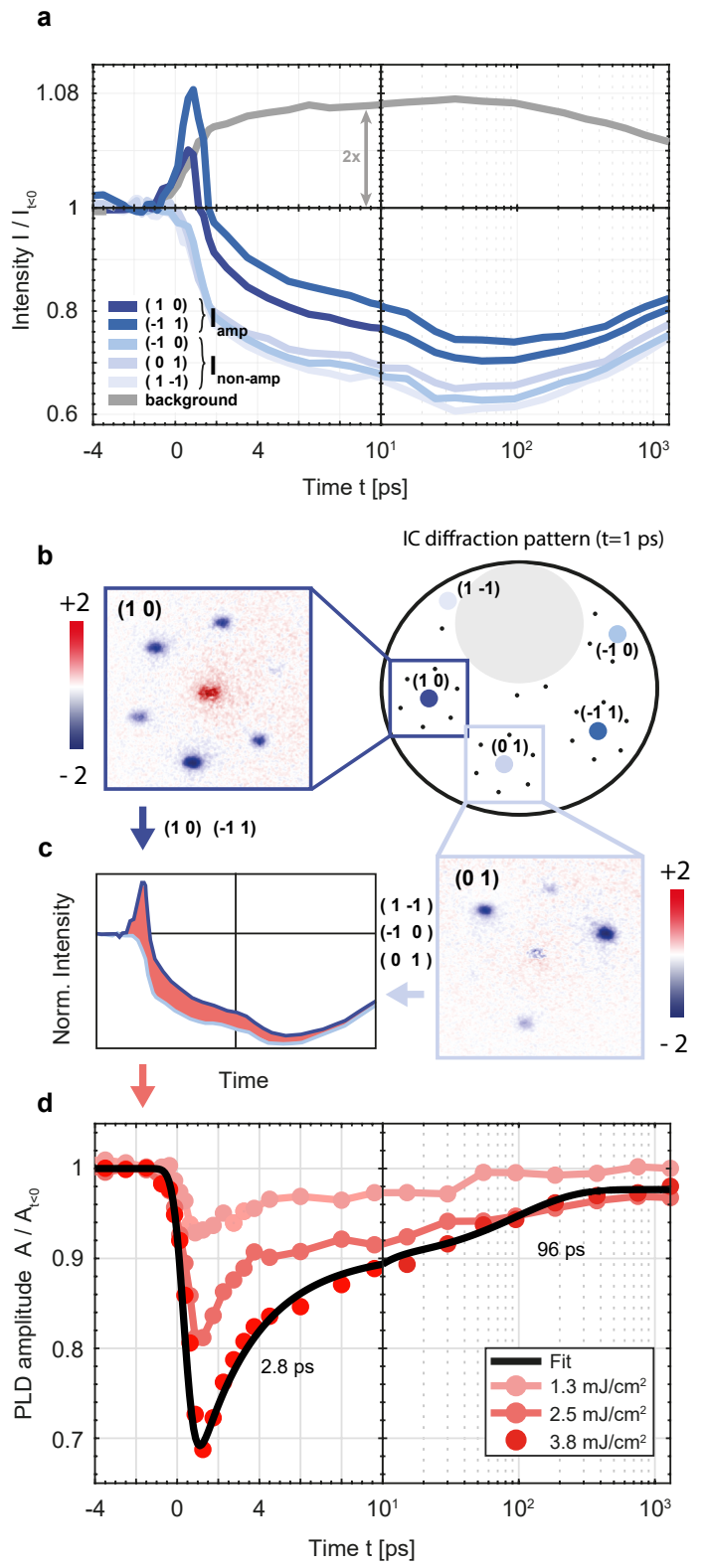

Figure 3.3: Amplitude dynamics of the PLD obtained from main lattice reflexes. (a) Time-dependent intensity of visible main lattice reflexes and integrated background intensity, for a fluence of $F=3.8 \mathrm{~mJ} / \mathrm{cm}^{2}$. Two inequivalent classes of spot groups are found, featuring a strong (dark blue) and weak (light blue) sensitivity to the amplitude quench. (b) Sketch of IC diffraction pattern, and parts of the difference diffraction image $\left(I_{t=1 \mathrm{ps}}-I_{t<0}\right)$ around the (10) and (01) main reflexes (insets). (c) Schematic comparison of peak intensities in the spot groups. Red area highlights different sensitivities to the PLD. (d) Extracted PLD amplitude quench and relaxation (see also Appendix 3.6.1) for three fluences, showing a rapid and a slower relaxation component. (Time constants from a biexponential fit (black line) to the highest fluence data: $(2.8 \pm 0.3) \mathrm{ps}$ and $(96 \pm 3) \mathrm{ps})$. 
the PLD by the two groups of main lattice peaks. Moreover, the simulations predict an energydependent and strongly reduced PLD-sensitivity at an electron energy of $80 \mathrm{eV}$. Indeed, experiments at this lower energy show that the transient increase of the main peak is generally much weaker (see additional data in Appendix 3.6.3).

We employ these different sensitivities to the PLD to derive a phonon-corrected amplitude signal. Specifically, we remove the phonon-induced Debye-Waller suppression by normalizing the intensity of the PLD-sensitive peaks to that of the weakly sensitive peaks (Fig. 3.3c; see Appendix 3.6.1 for details). The resulting phonon-corrected amplitude suppression is displayed in Fig. 3.3d for three pump fluences. In each case, the amplitude exhibits a rapid initial quench (within our temporal resolution), and a recovery with an exponential time constant of about 3 ps. The re-establishment of the amplitude is, however, incomplete, slowing down considerably beyond $4 \mathrm{ps}$, and lasting well into the range of tens to one-hundred picoseconds.

\subsubsection{Amplitude Analysis based on Satellite Reflexes}

We now aim at characterizing the evolution of the mean amplitude based on the satellite peak intensities, again removing a time-dependent phonon Debye-Waller factor. To this end, we compare the intensities of the main peaks with weak PLD-sensitivity to the satellite peaks. In Fig. 3.4a, we plot the logarithm of these intensities (normalized to the signal at $t<0$ ), divided by the fluence. For all three fluences, the traces of the main lattice peaks collapse to a single universal curve (blue), illustrating the phonon-induced Debye-Waller suppression $W_{\mathrm{s}}$ and its proportionality to fluence. The satellite peaks show a non-exponential fluence dependency in their suppression and recovery. At low fluences, however, where only a minor amplitude quench is induced, the satellite peak suppression closely follows that of the main peaks. We use this information to derive a phonon-corrected amplitude signal from the satellite peaks (see Appendix 3.6.1). Figure 3.4b shows the resulting amplitude evolution. For this graph, the satellite intensities were integrated over circular masks in the diffraction pattern (width of $\Delta k_{\mathrm{sat}}=0.36 \AA^{-1}$ ), therefore including also electrons scattered by a small angle from the reflex. We find a very similar behavior as from the main peak analysis (see Section 3.3.1), namely a rapid and fluence-dependent quench, a fast initial recovery and a rather persistent partial suppression, and we therefore consider this quantity as representative for the evolution of the amplitude $A$.

A somewhat different curve is obtained by utilizing not the area-integrated intensity, but the maximum intensity on top of the diffraction spot (bottom graph in Fig. 3.4b). Whereas the maximum and integrated intensities behave similarly at low fluence, at the highest fluence, the suppression of the maximum intensity exceeds that of the integrated intensity (grey curve from integrated intensity 
a

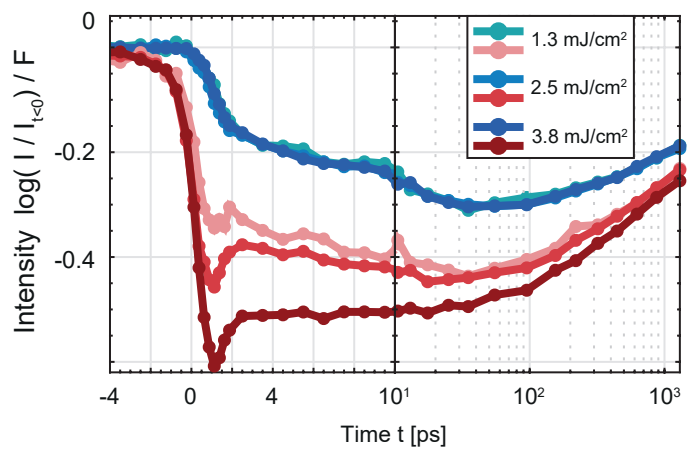

b $\quad \downarrow$ Phonon correction $\downarrow$

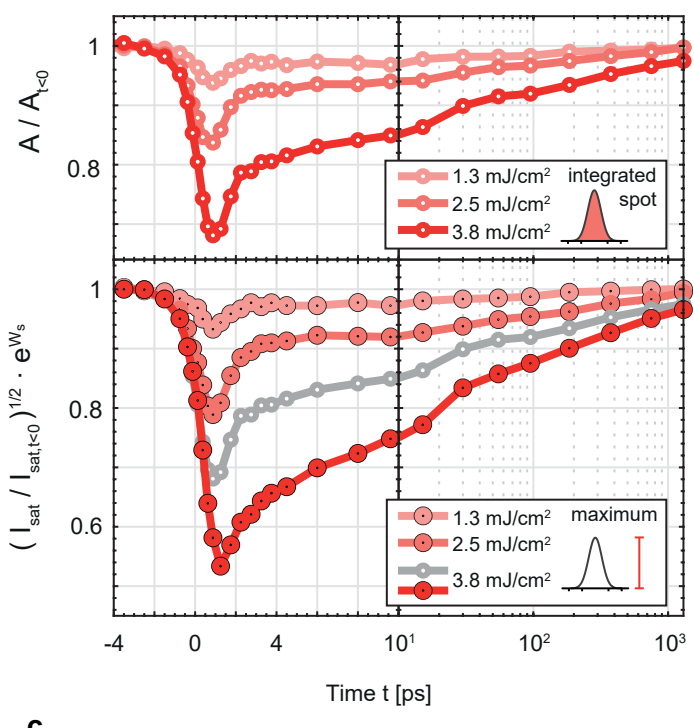

C

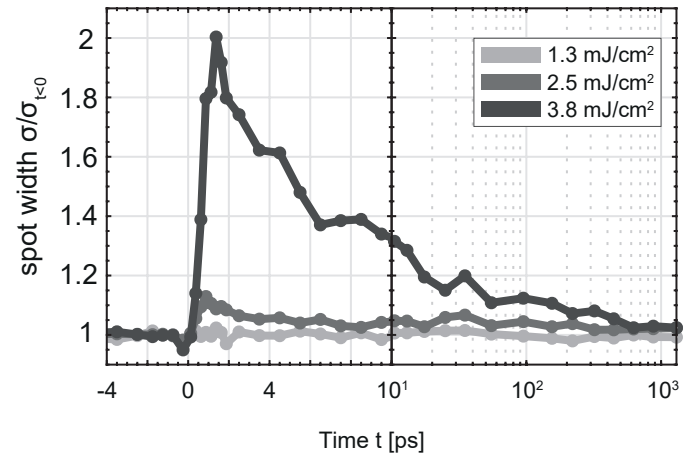

Figure 3.4: Amplitude dynamics of the PLD obtained from satellite reflexes. (a) Logarithm of normalized main lattice and satellite peak intensities (mean value), divided by fluence, versus time delay. While the main peak intensities (blue) collapse to a single curve due to the exponential (in fluence) Debye-Waller-type suppression, the satellite intensities (red) show a strong fluence-dependent behavior for early times, before converging for long time delays. (b) Phonon-corrected PLD amplitude obtained from integrated (top) and maximum (bottom) satellite intensities. (c) Fluence-dependent azimuthal spot width $\sigma$ versus time. 
shown again for comparison). Moreover, the recovery of the maximum proceeds more gradually than the integrated intensity.

The difference between the evolution of the integrated and maximum intensities implies a change in diffraction peak shape, which is analyzed in Fig. 3.4c. Plotting the azimuthal width of the diffraction peak, we find a significant time-dependent broadening for the highest fluence.

This effective broadening may be a result of several phenomena: (i) Diffuse scattering to the wings of the peak by low-energy phase excitations [147] will suppress the reflex maximum via the phason Debye-Waller factor $\exp \left(-2 W_{\varphi}\right)$ while largely maintaining the integrated intensity. (ii) An overall peak broadening from reduced correlation lengths will arise from the generation of CDW dislocation-type topological defects [14,38]. Except for the amplitude suppression in the dislocation core, this broadening also preserves the integrated intensity. At this point, we cannot rule out either scenario, and a more detailed spot profile analysis or higher momentum resolution may be required to further elucidate the different contributions.

\subsubsection{Non-equilibrium dynamics of the amplitude}

The incomplete recovery and persistent suppression of the PLD amplitude, independently obtained from the main (Fig. 3.3d) and satellite (Fig. 3.4b) reflexes, warrants further investigation. It implies that the system is either thermalized at a higher temperature with reduced equilibrium amplitude [194], or, alternatively, that non-equilibrium dynamics inhibit the recovery of the order parameter. It was previously suggested for the $\mathrm{NC}$ phase that the rapid recovery results in a thermalized system at elevated temperature [24]. Specifically, this would entail equilibrium between the electronic and different structural degrees of freedom after approximately 4 ps.

As shown in the following, we have evidence for a sustained non-thermal suppression of the order parameter. In Fig. 3.5, we consider in more detail the path to thermal equilibrium. An instructive depiction is obtained by plotting the main and satellite intensities against each other, resulting in cyclic trajectories in a two-dimensional plane (Fig. 3.5a), traced out over time in a clockwise fashion. At long delays (beyond $100 \mathrm{ps}$ ), the curves for all fluences follow a universal path (dashed line) representing a thermalized system at elevated temperatures, cooling down. Different trajectories reach the same combination of intensities at different times. For instance, the high-fluence trajectory exhibits the same combination of intensity suppressions at $1500 \mathrm{ps}$ as the intermediate fluence at a somewhat earlier time of 290 ps (black circle in Fig. 3.5a). Once the trajectory reaches this line, the surface is in local thermal equilibrium, characterized by a single temperature, and the satellite peak suppression is composed of a Debye-Waller factor as well as a thermal reduction of the amplitude. The further progression of the system, i.e. its cooling, is governed by thermal diffusion to the bulk. 


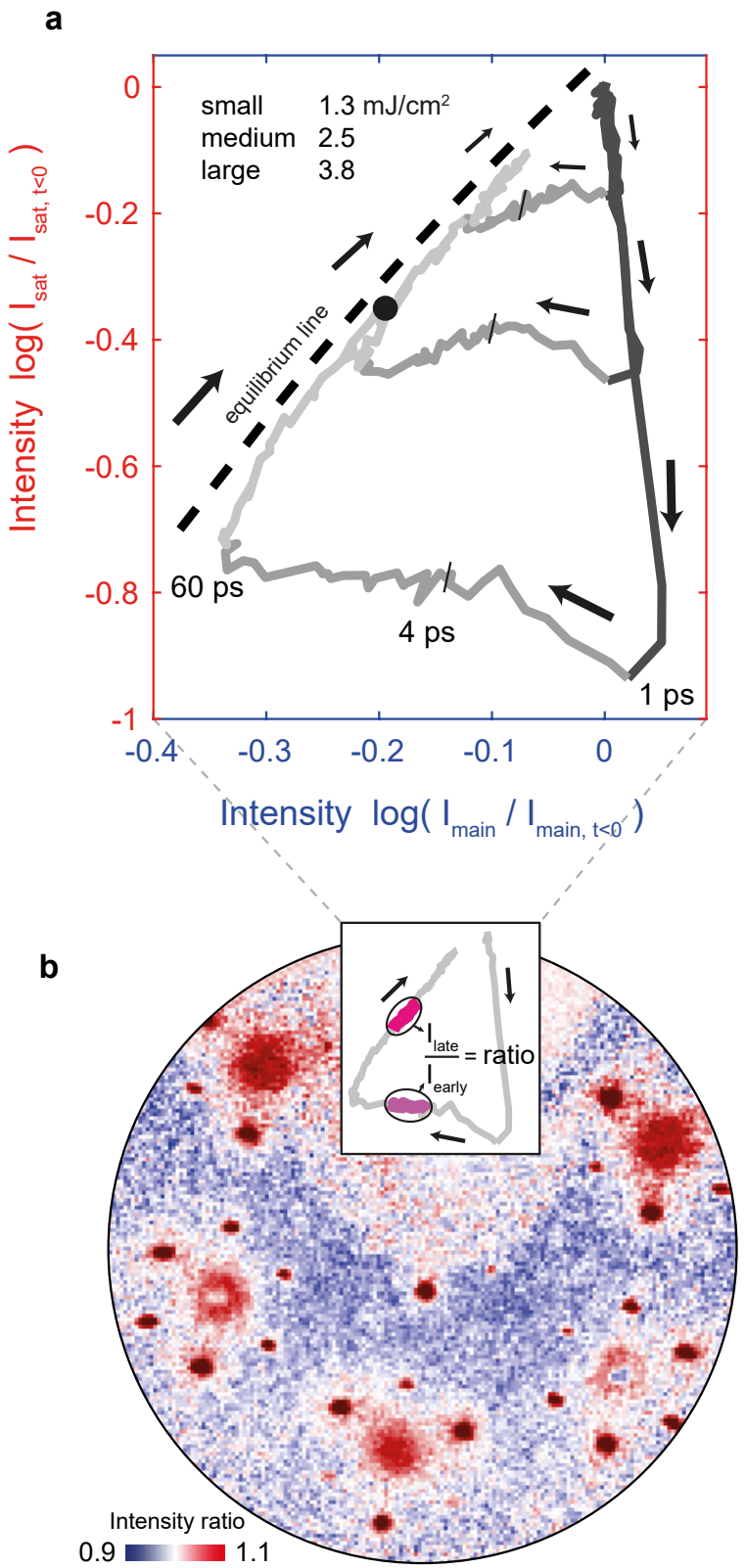

Figure 3.5: Path to equilibrium. (a) Intensities of satellite and main peaks (with PLD sensitivity) plotted against each other, leading to cyclic trajectories in a 2D plane with varying size. Note that all curves reach a common equilibrium line after approximately $60 \mathrm{ps}$. The gray color scale highlights certain time intervals (dark gray: 0-1 ps, intermed. gray: 1-60 ps, light gray: 60-1500 ps). The same combination of intensity suppressions is found for different fluences at different times (black circle corresponds to $1500 \mathrm{ps} / 290$ ps at high/intermediate fluence). (b) Ratio of time-integrated frames exhibits prominent pedestals around diffraction peaks, pointing to an enhanced acoustic phonon population on the equilibrium line. Late frames (dark magenta in inset, $t=790 \ldots 1500 \mathrm{ps}$ ) divided by early frames (light magenta in inset, $t=4.5 \ldots 10 \mathrm{ps}$ ). 
All points displaced from the dashed line represent deviations from a thermal state, with the distance being a very sensitive measure of the structural non-equilibrium. For example, within the first picosecond after the excitation (dark segments of the curves), the rapid quench of the order parameter causes a reduction of satellite intensity and a moderate enhancement of the main lattice signal, with a fluence-dependent maximum displacement from thermal equilibrium (corresponding curves for the main peaks insensitive to the amplitude are found in Appendix 3.6.4). The recovery to the thermal state now proceeds through various stages and in a fluence-dependent manner. After about 4 ps (see marks), the fast component of the amplitude recovery is completed (cf. Fig. 3.3d, compare also Ref. 74) [246]. However, the system remains far from the equilibrium state, i.e., exhibits a lower-than-thermal satellite intensity. Interestingly, for all curves, a surprisingly long time of approximately $60 \mathrm{ps}$ is required to reach the thermal state. This depiction directly shows that the persistent amplitude suppression discussed in Fig. 3.3d and 3.4b is in fact not of a thermal nature, and that we have a pronounced deviation from equilibrium between the degrees of freedom affecting the diffraction intensities.

To identify the origin of this long-lived amplitude suppression, we first note that the time at which the system reaches a thermal amplitude nearly coincides with the strongest suppression of the main lattice peaks. As this time also corresponds to the maximum intensity of the diffuse background (cf. Figs. 3.2d, 3.3a), the full equilibration of lattice fluctuations appears to be critical in controlling the structural order parameter. In particular, this lattice equilibration induces a significant increase of diffuse background intensity around main lattice peaks (difference image in Fig. 3.5b), directly pointing to the excitation of low-energy acoustic modes near the center of the Brillouin zone.

\subsection{Discussion}

Taken together, these observations indicate the sequence of relaxation processes illustrated in Fig. 3.6b, which can be related to the intensity curves (Fig. 3.4a) and the cyclic trajectories introduced above (simplified sketch in Fig. 3.6a). Within the first picosecond, optical excitation of the electronic system leads to a CDW amplitude quench and a strong deformation of the potential energy landscape (see insets in Fig. 3.6b), which triggers cooperative motion of the lattice towards its unmodulated state, including the excitation of coherent amplitude modes (stage 1) [34, 173]. Facilitated by the generation of high-energy lattice modes, the electron system cools down within few ps (stage 2), and as a result, the electronic potential and amplitude partially recover. The remaining PLD suppression in the following stage 3 strongly indicates a substantial population of CDW-coupled lattice excitations (Fig. 3.6b, red filling in bottom inset), such as amplitudons, phasons, and possible 
dislocation-type topological defects. Remaining non-thermal electronic excitations, on the other hand, can be largely ruled out at these late times, based on results from time- and angle-resolved photoemission spectroscopy [34, 187, 227, 230,247]. While it is known that phonon equilibration may take tens of picoseconds $[142,248]$, the present observations are significant in the sense that the persistent structural non-equilibrium is found to directly lead to an amplitude suppression via long-lived CDW-coupled excitations.

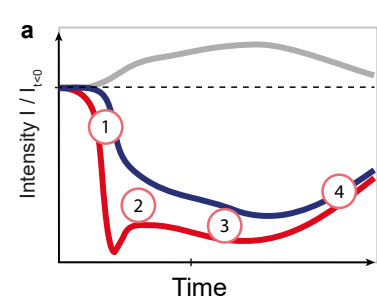

b

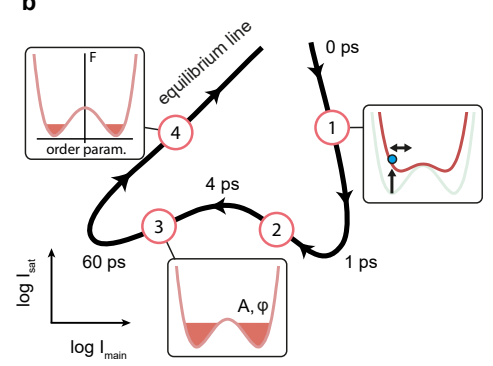

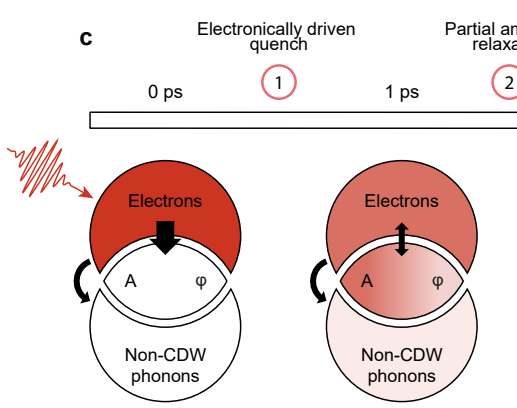

d $T_{e}>k_{b} \Delta$

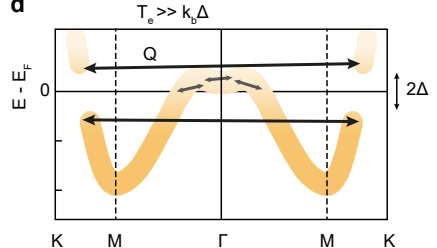

Partial amplitude
relaxation $\quad \begin{gathered}\text { Persisent amplitude } \\ \text { renormalization }\end{gathered} \quad$ Cooling

(2) $4 \mathrm{ps}$ (3) $60 \mathrm{ps}$
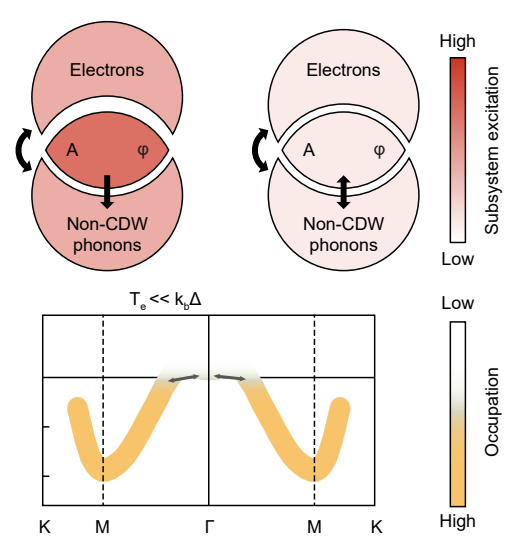

Low

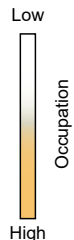

Figure 3.6: Linking relaxation pathways to CDW/PLD dynamics. (a) and (b) are simplified sketches of Figs. $2 \mathrm{~d}$ and $4 \mathrm{a}$, respectively, highlighting four phases of the relaxation process observed in the data. (c) Illustration of sequential relaxation process and the excitation levels of the three subsystems. The color shade represents energy content/temperature, black arrows indicate energy flow. (d) Simplified electronic band structure and populations (saturation of orange line) for high (left) and low (right) electronic temperature. Arrows indicate electron-lattice scattering processes. Scattering between gap regions (momentum transfer Q) is suppressed for reduced electronic temperatures.

Both amplitude and phase modes are expected to be rather efficiently excited by the optical pump, either directly by the deformation of the electronic potential (amplitude modes) [76] or by electron-lattice scattering between gap regions (Fig. 3.6d). Specifically, Fermi surface nesting is expected to result in a high probability of scattering events with a momentum transfer around the CDW wavevector $Q$ (Fig. 3.6d, left). Subsequent cooling of the carrier temperature below the energy scale of the electronic gap will effectively suppress these inelastic scattering pathways (Fig. 3.6d right) and decouple the subsystems (Fig. 3.6c), contributing to the persistent amplitude suppression in stage (3). Full lattice thermalization and the excitation of zone-center acoustic modes is then only achieved after $60 \mathrm{ps}$, from which point on the equilibrated system cools down (stage 4).

Let us consider the possible roles of different CDW excitations in the long-lived amplitude 
suppression, namely amplitudons, phasons, and CDW dislocation defects. Spatiotemporal variations of the amplitude and phase affect the observable value of $A$. Specifically, amplitudons represent amplitude oscillations $\Delta A$ around an equilibrium amplitude $A_{0}$, leading to an observed average value of $\left\langle A_{0}+\Delta A\right\rangle$. By an anharmonicity of the electronic potential, these oscillations become asymmetric, and a high population of amplitudons can reduce the value of $A$. In the case of phasons, despite early theoretical and experimental work [94, 95, 147-149, 152-154, 249], a unifying picture has not been reached, and recent assignments of their contribution in diffraction studies range from largely negligible [241] to dominant [36]. While our results do not definitely resolve this issue, the redistribution of scattering intensity near the satellite peaks suggests significant spatial or spatiotemporal phase distortions.

CDW dislocation defects should also be considered as a possible cause for the long-lived order parameter suppression, as they have been observed as a consequence of phase transitions, e.g. in $1 T-\mathrm{TaS}_{2}[14]$ or $\mathrm{LaTe}_{3}[38,193]$. The fact that we find a significant spot broadening of the satellites (Fig. 3.4c) most strongly at high fluences suggests a nonlinear dependence of phase fluctuations. This would be consistent with either CDW dislocations generated by critical phase fluctuations or a parametric decay of amplitudons into phase modes, as previously proposed [37].

\subsection{Conclusions}

The impact of fluctuations on symmetry breaking transitions has long been considered, for example in the Peierls instability [250-253]. Providing a time-domain view of the structural relaxation pathways, the present measurements highlight the impact of long-lived structural non-equilibrium to the order parameter.

The general mechanism of amplitude suppression by CDW-coupled modes should apply also to other phases and systems. Indeed, measurements in the NC phase feature a similar behavior as the IC phase (Appendix 3.6.2). Despite differences in symmetry, CDW wavevectors and electronic gaps, both phases exhibit closely related amplitude and phase excitations, as pointed out by Nakanishi and Shiba [115].

Relevant further questions pertain to the possible mechanisms of generating phasons and dislocation-type topological defects, as well as their coupling to regular lattice modes. Also the link between fluctuation modes and the creation and relaxation of metastable states [7,230] and the influence of partial and full commensurability in the different CDW states call for further investigation. Additional insights may be gained by investigating the ultrafast phase transitions between different CDW states $[14,189,190]$ and the populations of amplitude and phase modes in the nascent state 
after transition.

Considering methodical aspects, this work represents the first comprehensive study employing ultrafast low-energy electron diffraction with a temporal resolution of 1 ps. Future investigations using ULEED will enable a quantitative analysis of the three-dimensional structural evolution based on time- and energy-dependent diffraction. Moreover, the method is applicable to a wide variety of other surface systems and low-dimensional structures, harnessing its strengths of high momentum resolution, efficient scattering and enhanced sensitivity to lattice fluctuations.

\section{Acknowledgments}

This work was funded by the European Research Council (ERC Starting Grant 'ULEED', ID: 639119) and the Deutsche Forschungsgemeinschaft (SFB-1073, project A05). We gratefully acknowledge insightful discussions with H. Schwoerer, B. Siwick, J. D. Axe and T. Aslanyan. We thank L. Hammer for introducing us to the dynamical LEED computations. Furthermore, we thank K. Hanff for help with sample preparation.

\subsection{Appendix}

\subsubsection{Methods}

\subsubsection{Experimental details}

Here, we briefly describe our experimental ULEED apparatus (Fig. 3.7). The time-resolved measurements are conducted in an ultra-high vacuum chamber (base pressure $p=5 \cdot 10^{-11}$ mbar) into which samples are transferred via a load-lock system and cleaved in-situ. Inside the chamber, the electron source (microgun [47]) and a microchannel plate detector are mounted. A cooled CMOS camera records the detected electron diffraction patterns from outside the UHV chamber.

A femtosecond laser system (Amplifier, NOPA and OPA) generates three femtosecond laser beams of different center wavelength. An ultraviolet beam (center wavelength of $400 \mathrm{~nm}$ ) is focused on a nanometric tungsten needle that is embedded inside the microgun [47] generating ultrashort electron pulses via two-photon photoemission. An electrostatic lens assembly controls the collimation of the electron beam having an energy range of 40-100 eV. With a gun front diameter of $80 \mu \mathrm{m}$ and a working distance of around $150 \mu \mathrm{m}$, we achieve a temporal resolution of $1 \mathrm{ps}$ and an electron beam diameter of approximately $10 \mu \mathrm{m}$ at the sample. An upper limit of the technique's temporal resolution is obtained by the derivative of the fastest intensity change in our data set (suppression of satellite peak at $100 \mathrm{eV}$ ) shown in Fig. 3.1g. An infrared beam (1030 nm center 


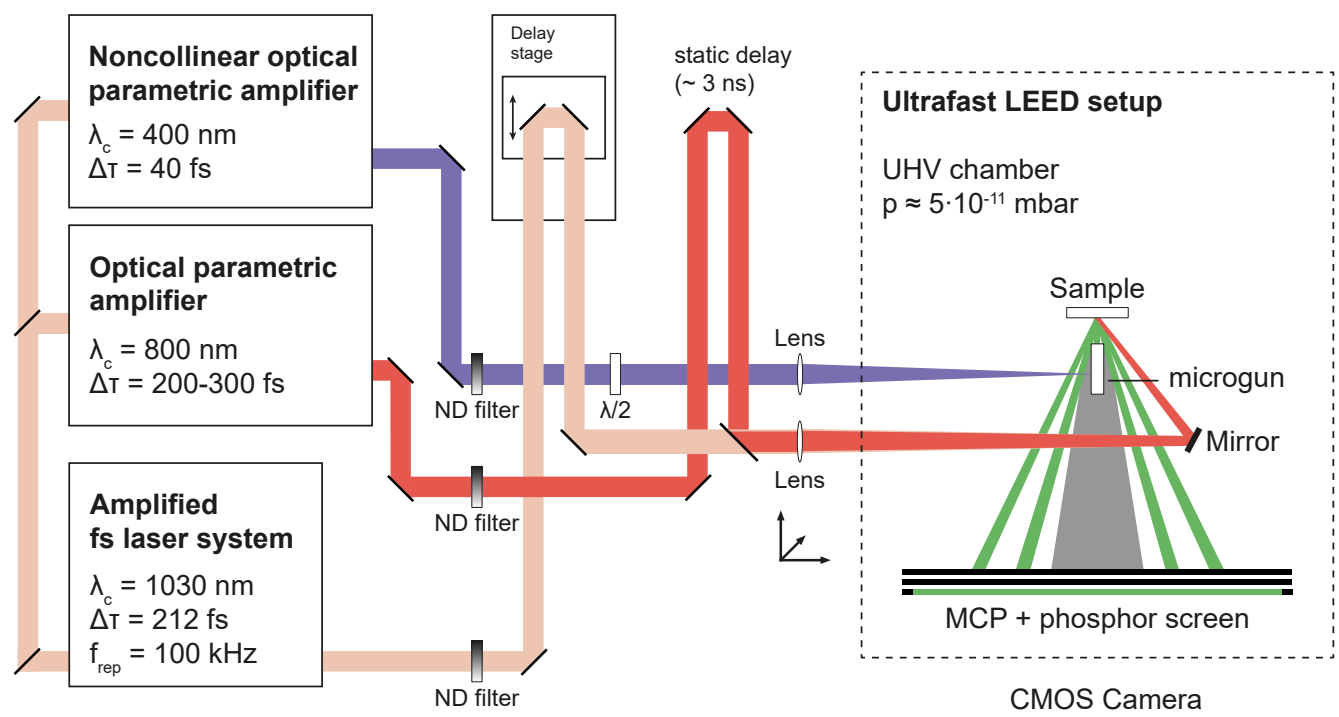

Figure 3.7: Schematic of the ultrafast LEED setup.

wavelength) optically excites the sample at specific times controlled by an automated linear delay stage. In order to heat the $1 T$-TaS 2 sample and stabilize it in the IC phase slightly above $T \approx 353 \mathrm{~K}$, a third beam $(800 \mathrm{~nm})$ is aligned collinearly with the infrared beam. This pulse is delayed by about $3 \mathrm{~ns}$ with respect to the electron and $1030 \mathrm{~nm}$ pulses (i.e., it arrives $10 \mu$ s before the next pulses) and thus leads to an average increase in sample temperature.

\subsubsection{Data analysis}

The recorded LEED images are preprocessed to correct for minor drifts in-between measurement runs, and for distortions caused by local electromagnetic fields as well as the projection to a flat MCP detector.

In order to obtain time curves (Fig. 3.2d) from the stacks of diffraction patterns, we process the data in a sequence of operations. First, a binary circular mask is laid on top of each individual reflex (Fig. 3.2c, blue and red circle, diameter $\Delta k_{\text {main }}=0.6 \AA^{-1}$ and $\Delta k_{\text {sat }}=0.36 \AA^{-1}$ respectively) for each time delay. Second, we fit 2D Cauchy distributions (background: slope and constant offset) to the satellite reflexes and 2D pseudo Voigt profiles (background: slope and constant offset) to the 
main lattice reflexes, to determine a background profile and subtract it from each spot:

$$
\begin{aligned}
& C(x, y)=\frac{A}{2 \pi \sigma_{1} \sigma_{2}} \cdot\left(1+\left(\frac{x}{\sigma_{1}}\right)^{2}+\left(\frac{y}{\sigma_{2}}\right)^{2}\right)^{-3 / 2}+(a \cdot x+b \cdot y)+C, \\
& P V(x, y)=A \cdot\left(1+\left(\frac{x}{\sigma_{1}}\right)^{2}+\left(\frac{y}{\sigma_{2}}\right)^{2}\right)^{-3 / 2}+B \cdot e^{-\left(x / \sigma_{3}\right)^{2}-\left(x / \sigma_{4}\right)^{2}}+(a \cdot x+b \cdot y)+C .
\end{aligned}
$$

Here, the $\mathrm{x}$ and $\mathrm{y}$ axes correspond to the azimuthal and radial directions (with respect to the main peak) for a given spot. Third, from the background-corrected segment, the average and the maximum intensity (average over brightest $4 \%$ within a mask) are determined for each reflex within the mask. The remaining intensity outside the circular masks forms the integrated background. For improved signal-to-noise ratio, several spot curves are averaged, i.e the satellite curves represent the mean of the 11 brightest reflexes. Furthermore, from the 2D fit functions, we obtain the azimuthal $\left(\sigma_{1}\right)$ and radial $\left(\sigma_{2}\right)$ widths for each reflex (Fig. 3.4b).

\subsection{Debye-Waller-corrected amplitude signal}

Next, we describe the separation of the amplitude-quench-related intensity changes from DebyeWaller-type peak suppression for main lattice (Fig. 3.3d) and satellite reflexes (Fig. 3.4b).

The dynamical LEED simulations indicate that there can be considerable differences in the coefficients $c_{\mathbf{s}}$ (Eq. 3.3). Empirically, we find that for the IC phase, the $\left\{\left(\begin{array}{ll}0 & 1\end{array}\right),(-10),(1-1)\right\}$ peaks show a negligible influence of the initial quench $\left(c_{\mathrm{s}} \approx 0\right)$. In Fig. 3.3a, for each fluence, light blue $\left(c_{\mathbf{s}} \approx 0\right)$ and dark blue curves $\left(c_{\mathbf{s}}>0\right)$ are averaged $\left(I_{\text {non-amp }}\right.$ and $\left.I_{\mathrm{amp}}\right)$. We now use the two peak groups to extract the temporal evolution of $A$ by removing the time-dependent DebyeWaller suppression $e^{-2 W_{\mathrm{s}}}$ from the intensity of the peaks sensitive to the PLD, with a constant factor $C_{1}=0.81$ that accounts for the slightly different $W_{\mathrm{s}}$ of these peaks:

$$
I_{\text {ratio, main, } F}=\left(\frac{I_{\text {amp }, F}}{I_{\text {non-amp, } F_{1}}}\right)^{\frac{F}{F_{1}} \cdot C_{1}}=1-c_{\mathrm{s}} A^{2}
$$

The value of $C_{1}$ was determined by the main peak suppression at long delays (beyond $1 \mathrm{~ns}$ ) and the lowest fluence $\left(F_{1}=1.3 \mathrm{~mJ} / \mathrm{cm}^{2}\right)$, for which a negligible amplitude change is expected. The value of $c_{\mathbf{s}}$ for the amplitude-sensitive peaks is determined from the $A^{2}$-intensity dependence of the phonon-corrected satellite peaks (Eq. 3.3), evaluated at maximum suppression of the lowest fluence.

From the satellite reflexes (11 brightest spots), the corrected amplitude is obtained similarly using the Debye-Waller-dominated main lattice curve $I_{\text {non-amp, } F_{1}}$ for the lowest fluence with the factor $C_{2}=1.2$

$$
I_{\text {ratio, sat, } F}=\left(\frac{I_{\text {sat }, F}}{I_{\text {non-amp }, F_{1}}}\right)^{\frac{F}{F_{1}} \cdot C_{2}}=A^{2} .
$$




\subsection{Fitting of time constants}

The fit function in Fig. 3.3d is based on a step-like decrease followed by two exponential relaxations

$$
S(t)=1-\theta\left(t-t_{0}\right) \cdot\left(-A_{1}+A_{2} \cdot\left(1-e^{-\left(t-t_{0}\right) / \tau}\right)\right)+A_{3} \cdot\left(1-e^{-\left(t-t_{0}\right) / \tau_{2}}\right)
$$

where $\theta$ is the Heaviside function, $t_{0}$ time zero, $A_{1}, A_{2}$ and $A_{3}$ are the amplitudes and $\tau_{1}$ and $\tau_{2}$ time constants. The complete fit function is the convolution of $S(t)$ with a Gaussian (FWHM of $1 \mathrm{ps)}$ corresponding to the temporal resolution in our experiment.

\subsubsection{Data for nearly commensurate (NC) phase}

Figure 3.8 displays the analysis discussed above applied to the nearly commensurate phase. Similar features are found in the pump-probe curves for the main and satellite diffraction peaks as well as the background (Fig. 3.8c), the long-lived amplitude suppression (Fig. 3.8d-e) and the relaxation cycles (Fig. 3.8f). In particular, the two-stage amplitude relaxation process (first stage up to 4 ps, second stage up to $60 \mathrm{ps}$ ) is very pronounced at all fluences.

\subsubsection{Data at $80 \mathrm{eV}$ electron energy}

Figure 3.9 shows additional data recorded in the IC phase at $80 \mathrm{eV}$ energy. The main lattice peaks show a much weaker dependency on the PLD amplitude (Fig. 3.9b-d).

\subsubsection{Relaxation cycles for main peaks (-1 1), ( $\left(\begin{array}{ll}0 & 1\end{array}\right)$ and $\left(\begin{array}{l}1 \\ -1\end{array}\right)$}

Figure 3.10 shows the relaxation cycles in the IC phase as in Fig. 3.5a, using the intensities of the main lattice peaks (-1 1), (0 1) and (1 -1) without sensitivity to the PLD amplitude.

\subsubsection{Impact of CDW defects on peak width}

Here, we argue that our data rules out a linear scaling of CDW defect density with fluence and is only consistent with a nonlinear or threshold behavior. Assuming a linear relation of the defect density with the fluence $n \sim F$ and a correlation length $L \sim 1 / \sqrt{n}$ (see Ref. [14]), the defect-induced broadening should scale as $\sigma_{\text {top }} \sim 1 / L \sim \sqrt{n} \sim \sqrt{F}$. A doubling of the normalized peak widths $\sigma_{\text {tot }}=\sqrt{\sigma_{0}^{2}+\sigma_{t o p}^{2}}$ with respect to the instrument resolution $\sigma_{0}$ for the highest fluence would then imply considerably higher broadening values for lower fluences $\left(\sigma_{t o t}, 2.5=1.7\right.$ and $\left.\sigma_{t o t}, 1.3=1.4\right)$ than observed in our measurement (Fig. 3.4c). Experimentally, we find maximum broadening values at $t \approx 1$ ps of $\sigma_{\text {tot }, 3.8} \approx 2, \sigma_{\text {tot }, 2.5} \approx 1.3$ and $\sigma_{\text {tot }, 1.3} \approx 1$ for the highest, intermediate and lowest fluence, respectively (see Fig. 3.4c). Thus, we infer that the density of topological defects does not scale linearly with fluence. 

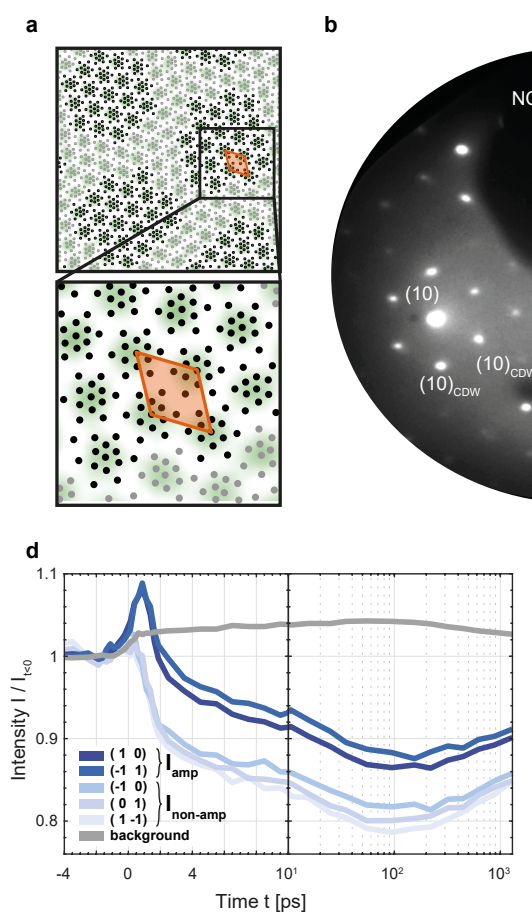

b

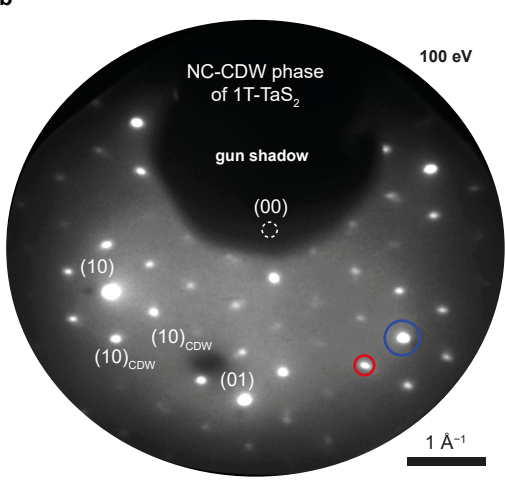

e

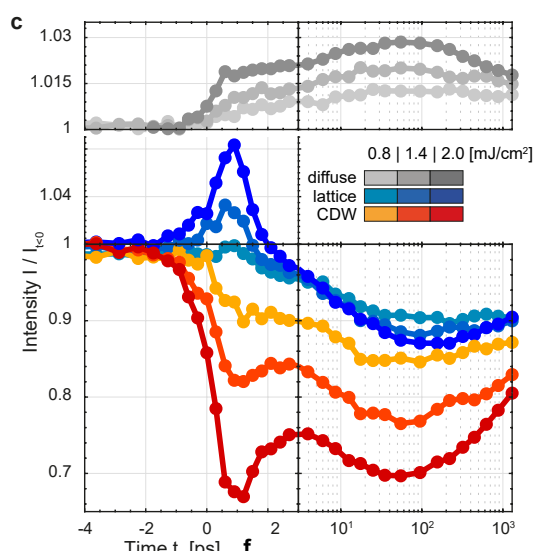

$\begin{array}{ccc}-4 & -2 & 0 \\ & \text { Time t }[p s] & \text { f }\end{array}$
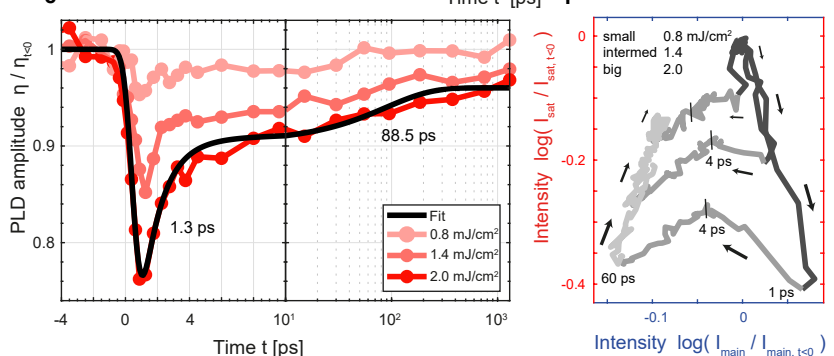

Figure 3.8: Measurements in the NC phase for an electron energy of $100 \mathrm{eV}$. (a) Top view of nearly commensurate (NC) CDW phase illustrating charge density (green), distorted lattice (black dots, displacements exaggerated) and superstructure unit cell (orange). (b) Diffraction pattern of the $\mathrm{NC}$ phase of $1 T-\mathrm{TaS}_{2}$ showing main lattice reflexes and several orders of PLD induced satellites (integration time: $90 \mathrm{~s}$ ). (c) Time-dependent measurement of reflexes (blue and red circles in (b)) and diffuse background (for three fluences). The main lattice signal is averaged over the (10) and (-1 1) spots (blue), the satellite signal over several reflexes. Curves are normalized to signal at negative times. (d) Time-dependent intensity of visible main lattice reflexes and integrated background intensity, for a fluence of $F=2.0 \mathrm{~mJ} / \mathrm{cm}^{2}$. Two inequivalent classes of spot groups are found, featuring a strong (dark blue) and weak (light blue) sensitivity to the amplitude quench. (e) Extracted PLD amplitude quench and relaxation for three fluences, showing a rapid and a slower relaxation component. (Time constants from a biexponential fit (black line) to the highest fluence data: $1.3 \mathrm{ps}$ and $88.5 \mathrm{ps}$ ). (f) Main lattice peak intensities vs. satellite peak intensities, leading to cyclic trajectories in a 2D plane with varying size. Following a two-stage relaxation, all curves reach a common equilibrium line after approximately 60 ps. The gray color scale highlights certain time intervals (dark gray: 0-1 ps, intermed. gray: 1-60 ps, light gray: $60-1500 \mathrm{ps})$. 

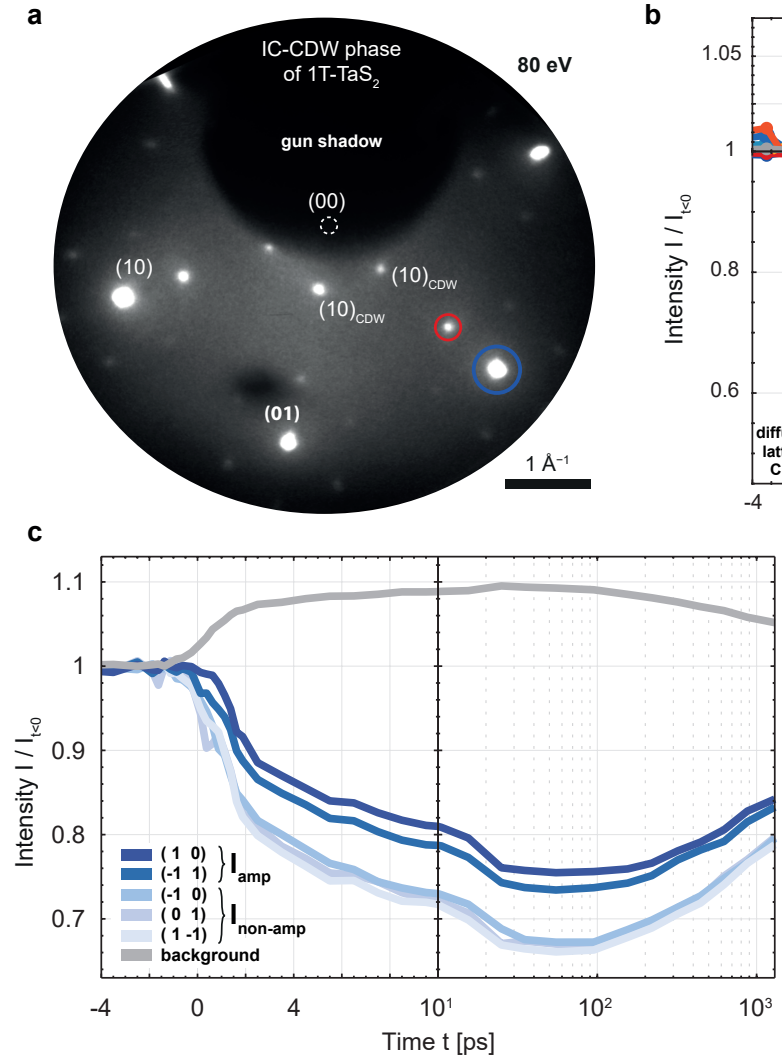

b

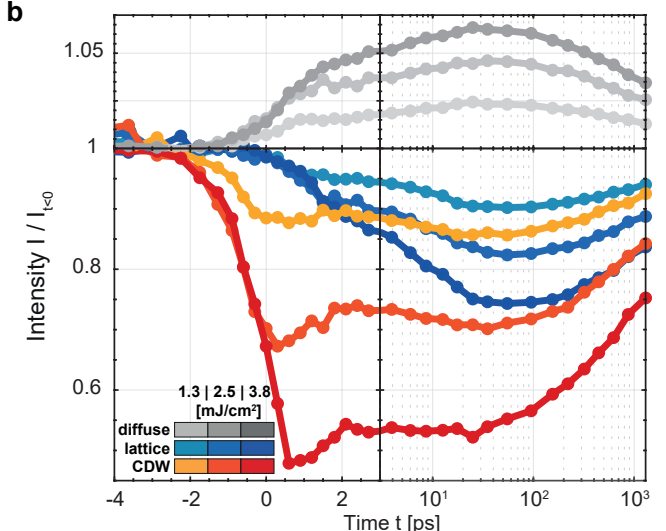

d

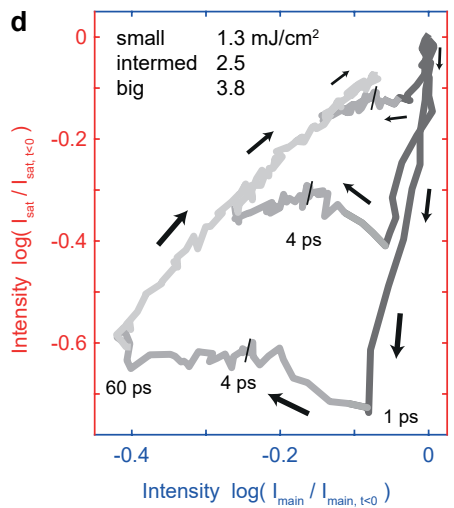

Figure 3.9: Measurements in the IC phase for an electron energy of $80 \mathrm{eV}$. (a) Diffraction pattern of the IC phase of $1 T-\mathrm{TaS}_{2}$ showing main lattice reflexes and first-order PLD-induced satellites (integration time: 90 s). (b) Time-dependent measurement of reflexes (blue and red circles in (a)) and diffuse background (for three fluences). The main lattice signal is averaged over the (10) and (-1 1) spots (blue), the satellite signal over several reflexes. Curves are normalized to signal at negative times. (c) Time-dependent intensity of visible main lattice reflexes and integrated background intensity, for a fluence of $F=3.8 \mathrm{~mJ} / \mathrm{cm}^{2}$. Two inequivalent classes of spot groups are found, but none shows a strong dependence on the amplitude quench. (d) Main lattice intensity vs. satellite peak intensity, leading to cyclic trajectories in a 2D plane with varying size. Note that all curves reach a common equilibrium line after approximately $60 \mathrm{ps.} \mathrm{The} \mathrm{gray} \mathrm{color} \mathrm{scale} \mathrm{highlights}$ certain time intervals (dark gray: 0-1 ps, intermed. gray: 1-60 ps, light gray: 60-1500 ps). 


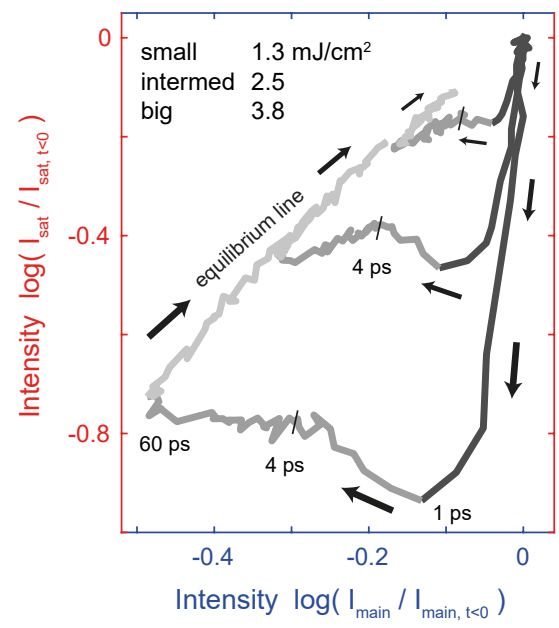

Figure 3.10: Main lattice peaks without amplitude feature vs. satellite peak intensities, leading to cyclic trajectories in a 2D plane with varying size. All curves reach a common equilibrium line after approximately $60 \mathrm{ps}$. The gray color scale highlights certain time intervals (dark gray: 0-1 ps, intermed. gray: 1-60 ps, light gray: $60-1500 \mathrm{ps})$.

\subsubsection{Dynamical LEED computation}

We performed dynamical LEED simulations on the commensurate CDW phase of $1 T-\mathrm{TaS}_{2}$, varying the atomic displacements of sulfur and tantalum continuously from the undistorted structure towards the C-phase structure recently reconstructed [129]. We are aware that the $\mathrm{C}$ phase is a simple approximation for the description of the high-temperature incommensurate CDW phase. However, it exhibits the same crucial feature of opposing sulfur displacements that we believe is responsible for the different sensitivities of the main lattice peaks. Also, dynamic LEED calculations involve high computational effort, in particular for large unit cells necessary for incommensurate structures. The obtained data contains PLD-amplitude- and energy-dependent scattering intensities for main lattice and CDW satellites spots. In the following, we focus on main lattice diffraction intensities.

In the electron energy range of $70-140 \mathrm{eV}$, the diffraction intensity is mainly determined by scattering from sulfur atoms, explaining the strong dependence from the PLD amplitude of sulfur atoms (Fig. 3.11a).

Figure 3.11b shows PLD dependent intensities for an electron energy of 100 and $80 \mathrm{eV}$, each normalized to the intensity value for zero distortion (metal structure). The PLD amplitude range is adapted to the expected values realized in the incommensurate phase [42] which are assumed to be considerably smaller ( $~ 30 \%$ of PLD amplitude of the commensurate low-temperature phase). In this range for $100 \mathrm{eV}$, we can show that there are two groups of main lattice spots that respond differently upon PLD change, whereas for $80 \mathrm{eV}$, all intensities follow a common curve. Moreover, 
the magnitude of the relative intensity changes approximately matches the observed ones in the experiment. The curves within a group of main lattice peaks $\left\{\left(\begin{array}{ll}1 & 0),(-1\end{array}\right)\right\}$ and $\{(-10),(01),(1$ -1) \} conincide since the simulation is performed at normal incidence.

Figure 3.11c shows energy-dependent intensity curves for two main lattice peaks contained
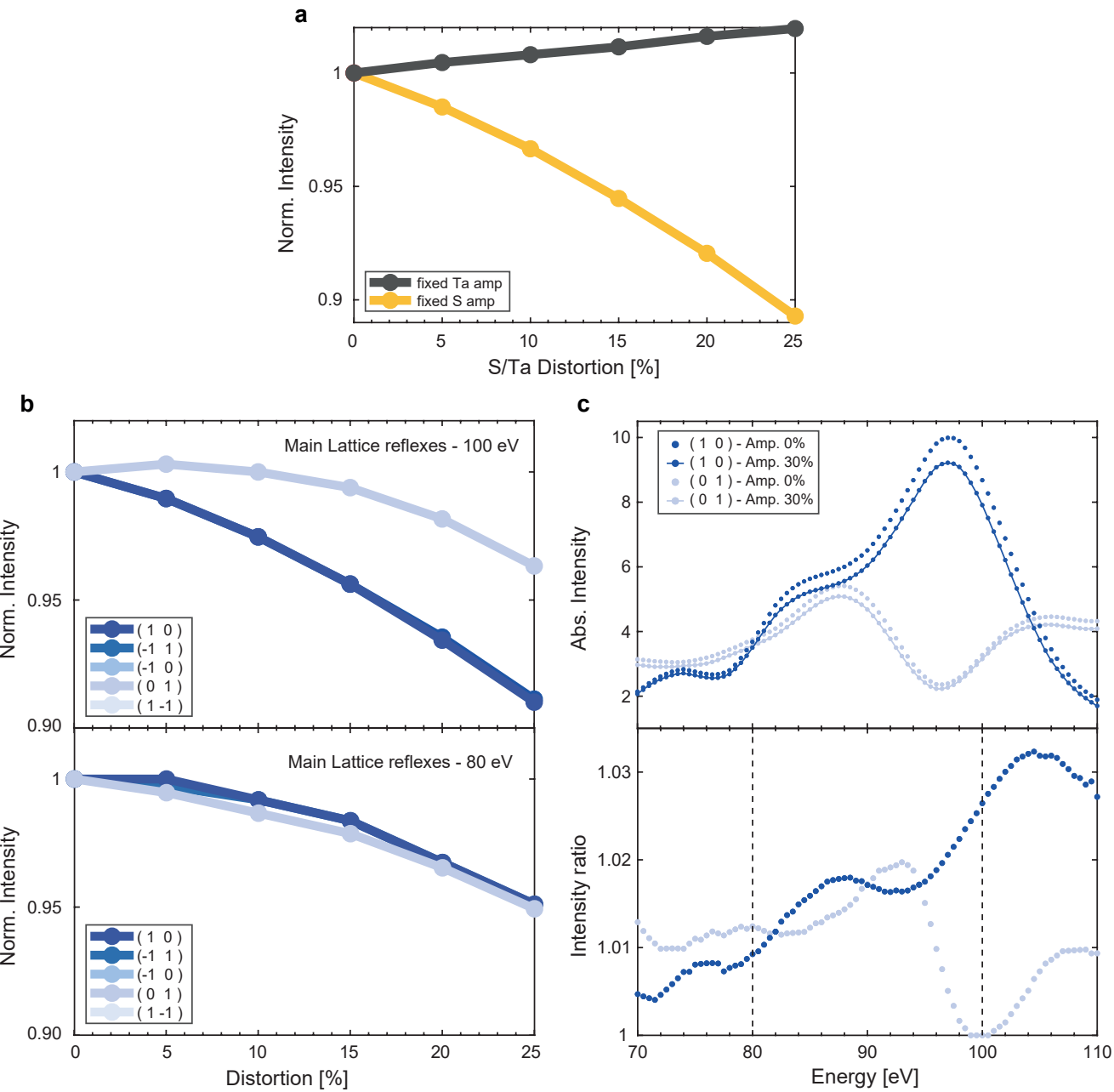

Figure 3.11: Dynamical LEED simulations. (a) Normalized intensity of main lattice reflex (ll 0 ) as a function of sulfur and tantalum displacement for an electron energy of $100 \mathrm{eV}$. Enhanced scattering off sulfur atoms results in a much stronger dependence on the sulfur atom displacements. (b) Normalized intensities of main lattice spots for an electron energy of 80 and $100 \mathrm{eV}$ as a function of the fraction of the maximum commensurate PLD amplitude. The diffraction reflexes split up into two spot groups. Light and dark blue curves coincide, respectively, due to normal incidence of the electron beam. (c) LEED spectra (top) for both groups (light and dark blue) for vanishing (points) and finite (dash points) distortion. The percentage refers to the amplitude of the commensurate PLD in low-temperature phase. The intensity ratio (bottom) illustrates the energy-dependent sensitivity between reflex groups. 
in one of the two groups (light and dark blue), each for zero PLD and 30\% PLD amplitude of the commensurate low-temperature phase. The ratio of spectra for each spot with minimal and maximal amplitude (Fig. 3.11c, bottom) displays a rich oscillatory behavior. Importantly, however, for energies of $80 \mathrm{eV}$ and $100 \mathrm{eV}$, the spots exhibit a drastically different sensitivity to PLD changes, with a small and large difference for the separate spot groups, respectively. These predictions directly corroborate our experimental findings at the different electron beam energies. 


\section{4 | Discussion and Conclusions}

In the following, a brief summary of the previous two chapters is given. After this, the current technological status of ultrafast LEED are discussed and possible future perspectives for more detailed work on CDW or CDW-related materials systems are pointed out.

\subsection{Summary of Publications}

Chapter 2 reports the design and fabrication of a micrometer-scale electron gun for the implementation in ULEED experiments. The fabrication process consists of multiple steps, including the preparation of a suitable support for electrical contacts by means of photolithography, as well as the construction of the gun assembly, the nanometric tip emitter and the shielding using focused-ionbeam (FIB) etching. We achieve an effective gun diameter of $80 \mu \mathrm{m}$, allowing for a considerably shorter propagation distance to the sample in the diffraction experiment as compared to the previous minigun design.

Finite element simulations enable us to estimate the gun performance. A large number of particles, each having different initial conditions, are propagated to obtain the trajectories in the electric field determined by the gun geometry. At a distance of $400 \mu \mathrm{m}$ behind the ground aperture, the pulse duration is extracted from the differences in time of flight within the ensemble of particles for an energy range of 40-100 eV, yielding values of 1 ps or less for most electron energies.

Finally, the pulse duration and the momentum resolution are characterized experimentally. For the measurement of the pulse duration, the electron pulses traverse a time-dependent electric field that forms when an intense laser pulse hits a metal grid. From the transient change of the projected image, we obtain a pulse duration of $1.3 \pm 0.2$ ps. LEED images of $1 T-\mathrm{TaS}_{2}$ demonstrate the achievable momentum resolution yielding a transfer width of $25 \mathrm{~nm}$ and a beam emittance of $200 \mathrm{~nm}$ rad.

In chapter 3, the microgun is employed to study the non-equilibrium lattice dynamics of the charge-density wave material $1 T-\mathrm{TaS}_{2}$ with $1 \mathrm{ps}$ temporal resolution. In the nearly commensurate 
and incommensurate CDW phase, we trace the time-dependent intensity of main and satellite reflexes as well as the diffuse background. The intensity redistribution within a diffraction pattern is governed by changes of the average PLD amplitude, CDW-related fluctuation modes and non-CDW phonons.

We use two approaches in order to separate the dynamics of the structural order parameter from the phonon population. We compare the different intensities of two inequivalent classes of main lattice reflections to the PLD, and exploit the direct sensitivity of the satellite reflexes to the PLD. When we apply these methods to our data, we obtain the transient non-equilibrium signal of the average PLD amplitude that recovers over the course of about 60 ps.

The observations suggest a sequence of processes that include the laser-induced heating of the electronic system, efficient coupling to CDW-coupled modes, lattice equilibration, and cooling of the thermalized state. We assign the long thermalization time of the order parameter to the presence of a hot population of long-lived CDW-coupled excitations. For strong laser excitation, the broadening of superlattice satellite reflexes indicates the presence of dislocation-type CDW defects that additionally reduce the PLD in the defect core.

\subsection{Improving the Electron Source}

As described in Chapter 2 and 3, the microgun was developed and successfully implemented in ultrafast low-energy electron diffraction experiments [14, 49]. The need for miniaturization is rooted in the necessity to reduce the pulse broadening caused by energy dispersion (see Fig. 4.1). At the same time, it is important to collect as much of the backreflected electron signal as possible without blocking it by the electron gun. Before discussing possible alternative approaches to this problem, we focus first on the limiting factors and major obstacles in the gun fabrication process.

Challenges of a Microgun Any miniaturized electron gun should adhere to the following requirements:

1. Strongly confined emitter

2. Lens for beam collimation

3. Small front diameter

4. Efficient shielding

5. UHV compatibility

6. Electrical robustness

7. Mechanical robustness 
In this work, photolithography and FIB etching have been used in order to fabricate a device that fullfills the given requirements (see Chapter 2). The choice of these techniques, however, introduce limitations and inter-dependencies to several of the listed requirements. A full discussion of all details includes many technicalities which go beyond the scope of this section and are therefore kept to a minimum.

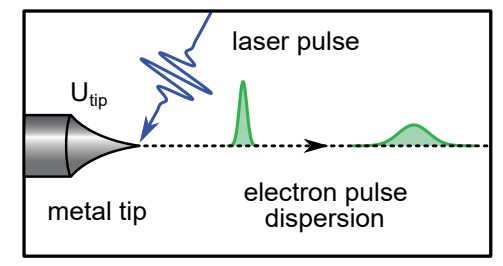

Figure 4.1: Electron pulse broadening due to a finite initial energy width.

The first four points are related to the specific geometry of the electron gun and therefore challenge the capabilities of the FIB technique. Generally, metal tips are known to be well suited as photoemitters because a very confined emission surface at the apex results in enhanced beam characteristics [128]. While FIB has been proven to be quite suitable for etching metal tips with apex diameters in the range of several of nanometers [254], the variety of electrode shapes and lens assembly geometries are, however, strongly limited. This is mainly due to the fact that the electron-optical elements should meet a high degree of rotational symmetry along the optical axis to ensure a sufficient electron beam quality. FIB etching perpendicular or along the symmetry axis maintains the overall symmetry whereas any deviation from these directions easily removes it. Additionally, the etching process might increase the surface roughness which can reduce the electrical robustness because of strong local electric fields and possible field emission.

The role of electrical shielding turned out to be of higher priority than initially expected. Diffracted low-energy electrons are highly susceptible to any stray fields leaking out of the electron gun or the support. In the presence of these fields, the diffraction pattern is strongly distorted, and the observable $k$-space is significantly reduced at short gun-sample distances (several $100 \mu \mathrm{m}$ ). Hence, electrical shielding should, on the one hand, fully suppress any stray fields, while also only marginally increase the front diameter.

One major issue intrinsic to the FIB technique concerns the deposition of platinum, which is necessary for the building process and electrical connections. During the deposition procedure, a small needle is positioned near the sample, through which a precursor gas is funneled into the vacuum chamber close to the sample. In order to grow a conductive connection, this precursor gas is locally cracked via intense secondary scattering that is induced by high-voltage ion beam irradiation. 
The radius of this deposition process lies in the range of several tens of micrometers, resulting in a large conductive metal film that interconnects all electrodes of the gun and the conductive metal strips on the glass support. Additionally, the problem is exacerbated when adsorbed remains of the precursor gas are cracked by the impact of the scanning electron beam. In order to reduce the effect of this emerging platinum halo on the sample, the dose of the incoming electron beam during each transfer step was reduced as much as possible, and the vacuum chamber was vented after every transfer step to remove any adsorbed precursor gas. Moreover, at crucial areas, the platinum halo was removed via ion-beam etching to electrically disconnect all electrodes and metal strip lines. Despite the efforts to minimize the impact of platinum halo, the achievable breakdown voltages for this device are drastically decreased by at least half compared to the raw support $(\approx 1000 \mathrm{~V})$, and the electrical robustness is significantly lowered.

The third point introduces very specific requirements on the multi-layer support as well as the subsequent photolithographic fabrication process (see also Fig. D.1). Firstly, a very thin substrate $(\sim 70 \mu \mathrm{m})$ minimizes the shadowing of the back-diffracted electrons, but complicates the handling during lithography and strongly reduces the mechanical robustness of the final device. Secondly, to ensure a minimal propagation distance between gun and sample, the metallic contact lines should converge in a small surface at a corner of the substrate. However, while lithography, in general, is an ideal tool for producing complicated nanostructures on a surface, it underperforms on edges and corners of substrates. To work around this issue, the lithographic process is carried out away from the edge of the substrate, and the substrate is cleaved as the final step. To this end, predetermined breaking lines of roughly half the thickness of the substrate are sawed into the glass support prior to the lithographic process step. However, this makes the glass substrates much more fragile and drastically complicates the handling during lithography. Although the cleaving procedure typically produces clean edges, their exact position varies on the order of several tens of micrometers, sometimes removing large parts of metallic strip lines.

The kinds of materials suitable for a pulsed LEED gun are limited, since we operate in ultra-high vacuum conditions. Specifically, very low outgasing rates are crucial to avoid contamination of the sample surface during a ULEED experiment. While metals ( $\mathrm{Au}, \mathrm{Ag}, \mathrm{Ti}, \mathrm{Cr}, \mathrm{W}, \mathrm{Pt})$ are usually not problematic, the class of electrically insulating materials is restricted to glasses, ceramics and certain polymers (polyimide and polytetrafluorethylen (Teflon)). Moreover, all materials have to be thermally stable and keep a low outgasing rate for temperatures of up to $150^{\circ} \mathrm{C}$ since UHV conditions are achieved via an intermediate baking-out process.

Taken together, the fabrication process comprises a large number of steps, after each of which it is crucial to check the fabrication quality. Nevertheless, it is very challenging to fully avoid 
sharp conductive edges and to control breakdown voltages, among other things. Consequently, some possibility of device failure remains. Combined with a total building time of several weeks, including intermediate gun tests in the vacuum chamber, the feedback loop for improving the production process is very long. During this thesis, the entire building process was completed about 25 times.

Future gun development might include improvements of electrical and mechanical robustness. More specifically, increasing the shortage voltage threshold would allow for higher electron energies and extraction fields. A wider electron energy range provides access to a wider range of a LEED spectrum, and higher extraction fields around the tip apex have a positive effect on the achievable pulse duration. It is also highly desirable to reduce the fragility of the instrument as it can be quickly damaged during handling outside the vacuum chamber and during measurements when working with the sample manipulator inside the vacuum chamber. In view of the above discussion, however, mechanical stability and miniaturization are virtually opposing concepts and very challenging to reconcile.

(a)

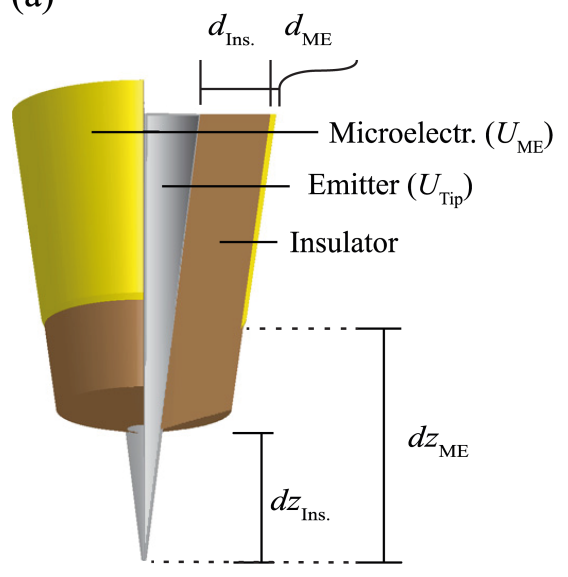

(b)

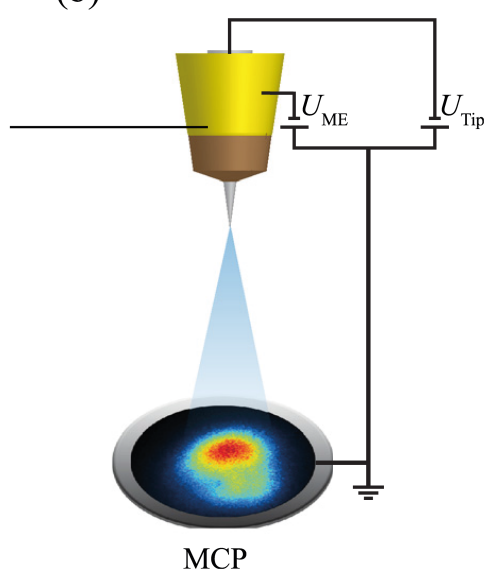

Figure 4.2: Illustration of (a) a compact microelectrode field emitter and (b) experimental setup. The multilayer coating of a tungsten nanotip is an elegant way for miniaturization. Reprinted from [216], with the permission of AIP Publishing.

Alternative Approaches We now discuss alternative ways to approach the task of fabricating a miniaturized source for low-energy electrons. A valid starting point is to rethink the design or topology of the device. The essential core elements determining the gun design proposed in this work are a nanometric tip, disk electrodes and conducting strip lines on a thin substrate. A very elegant design would combine the actual electrodes with the conductive metal contacts. A straightforward version of such an approach was published by Lüneburg et al., presenting a highly 
compact microelectrode-integrated field emitter. Figure 4.2 shows an illustration of the device that consists of a tungsten nanotip coated with a few micrometers thick polyimide film followed by a several nanometers thick gold film. The extension of this approach, however, would involve further conductive and insulating layers complicating the fabrication process on this curved surface even further. Moreover, in a back-reflection geometry, an efficient shielding is very important and probably challenging to achieve for this design.

(a)

(c)
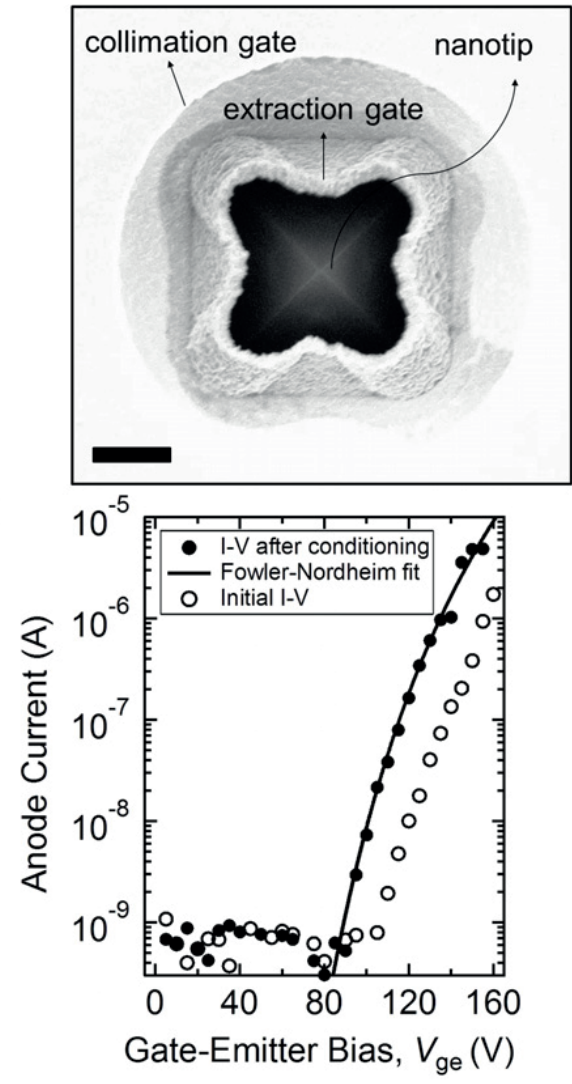

(b)

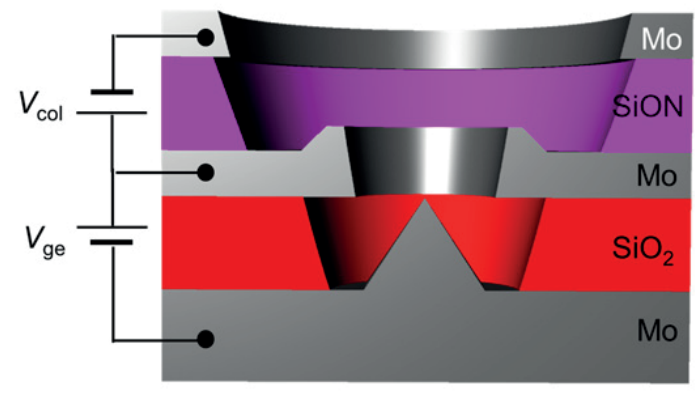

(d)

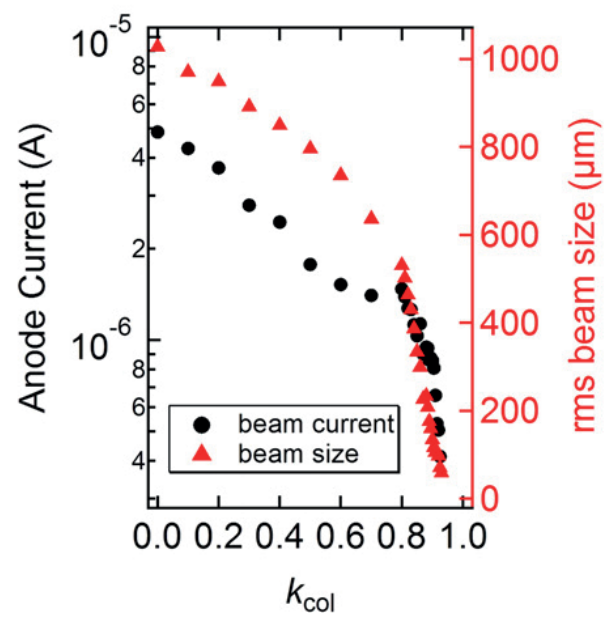

Figure 4.3: (a) Micrograph of the double-gate single nanotip field emitter device (top view). The scale bar denotes $1 \mu \mathrm{m}$. (b) Schematic illustration of the device, cutout of the side view. Characterization data in the field emission mode (c) and (d). Reprinted from [255], with the permission of AIP Publishing.

While Lüneburg's approach leans towards the topology determined by the nanometric tip, a different approach by Lee et al. focused on a design in the plane. As illustrated by Figure 4.3a and 4.3b, the device consists of a nanotip field emitter and two gates combined in a multilayer stack. The advantage of this design is that it inherits the strengths of lithography, including fabrication of well-defined nanostructures, reproducibility due to standardized process steps, and the capability to produce large numbers of devices simultaneously. There are considerable drawbacks of this design, 
however, for diffraction in a backscattering mode. Firstly, in this geometry, it is challenging to align a fs-laser onto the nanotip emitter for pulsed operation. Secondly, the device should be positioned at a corner of the substrate in order to minimize the shadowing of backscattered electrons. As discussed above, producing high quality nanostructures at edges and corners is a weakness of lithography.
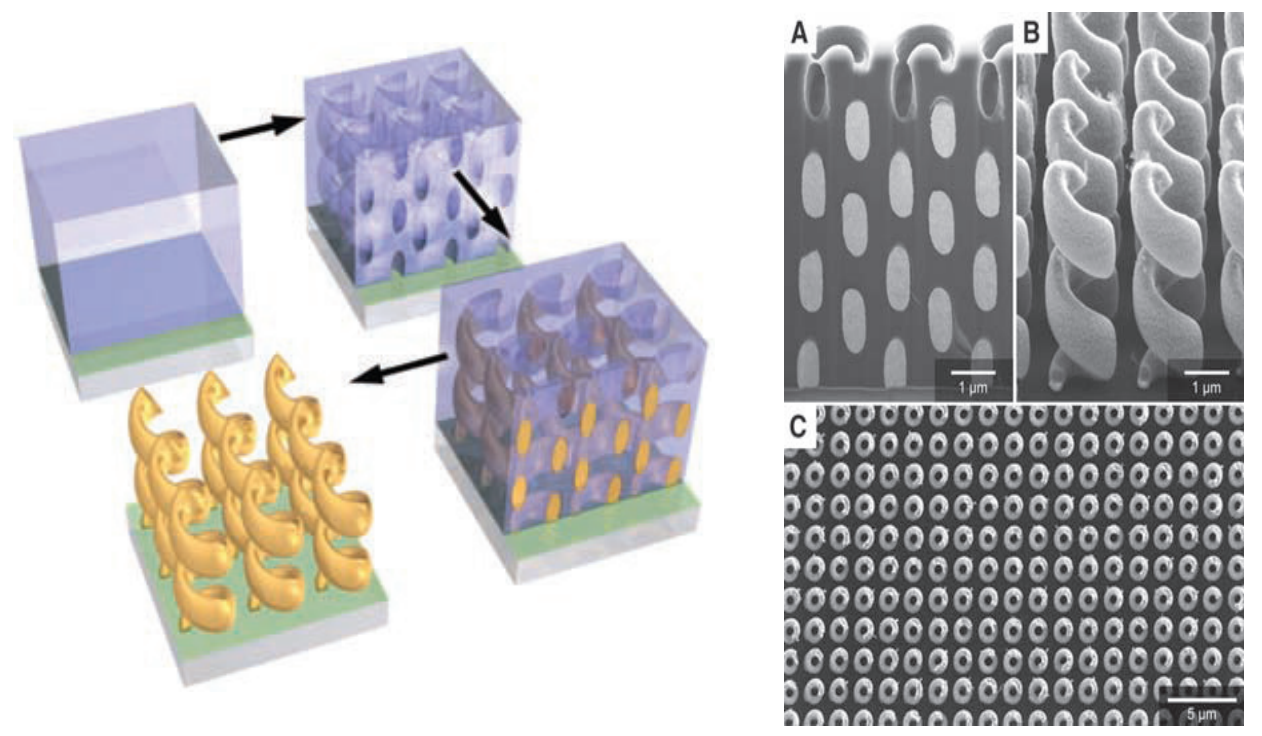

Figure 4.4: Fabrication of metal nanostructures using $3 \mathrm{~d}$ laser printing. Lithographic process including the fabrication of a template and the controlled growth of metal structures (left). Micrographs depicting high-quality gold helices in a large regular pattern (right A-C). From [256]. Reprinted with permission from AAAS.

Recently, it has been shown that lithography can also be performed in three dimensions by means of laser nanostructuring yielding outstanding resolution and precision [257]. The underlying mechanism is a multiphoton absorption process which can be used to drastically change the solubility of a photoresist in a very confined volume and thereby build structures on the scale of the light focus. To this day, however, this direct laser printing technique can only be used to process non-conductive polymers preventing direct access to $3 \mathrm{~d}$ metallic nanostructures. A seminal work from Gansel et al. extended the approach of laser nanostructuring and combined it with electroplating, achieving metal structures of unprecedented quality on the nanometer scale [256], as shown in Figure 4.4. More specifically, a laser beam writes helix shapes into a photoresist creating a template on a thin indiumtin oxide layer (see 4.4 left). Subsequently, by means of electroplating, gold is grown starting from the conductive layer and filling the void cavities of the negative template. As depicted in Figure 4.4 A-C, high-quality gold helices of about $1 \mu$ m diameter are grown on the substrate in a highly regular pattern. The challenge of this technique, however, lies in the control of the process parameters for fabricating the template and growing the metal structures. For a miniaturized electron gun, the weak 
points of the procedure may concern the growing of a nanometric tip as well as the minimization of surface roughness to avoid field emission from sharp edges when voltages are applied.

Besides miniaturization of the gun, there are further prominent strategies available to obtain short electron pulses at the sample plane. For example, radio-frequency electron pulse compression - or temporal refocusing - has been successfully employed for electron beams in the keV-regime to yield sub-100fs electron pulses [258, 259]. Figure 4.5 illustrates the basic principle of this technique, which is based on a dispersed electron pulse passing trough a time-dependent electric field. The top row shows the instantaneous state of the field and the position of the electron pulse, whereas the bottom row illustrates the momentary phase-space distribution. In the course of stage 1 through 3 , the transient electric field inside the cavity flips the configuration in phase space, such that fast electrons at the front of the pulse are decelerated, and slow electrons at the back of the pulse are accelerated resulting in an overcompressed pulse. In stage 4, the electron pulse freely propagates towards the sample and reaches its state of minimal longitudinal (z-direction) extension at the sample plane (stage 5). The temporal focus length can be tuned via the amplitude of the cavity field. A main challenge of this approach is a frequency- and phase-stable operation in order to minimize jitter and, as a result, the achievable pulse duration.
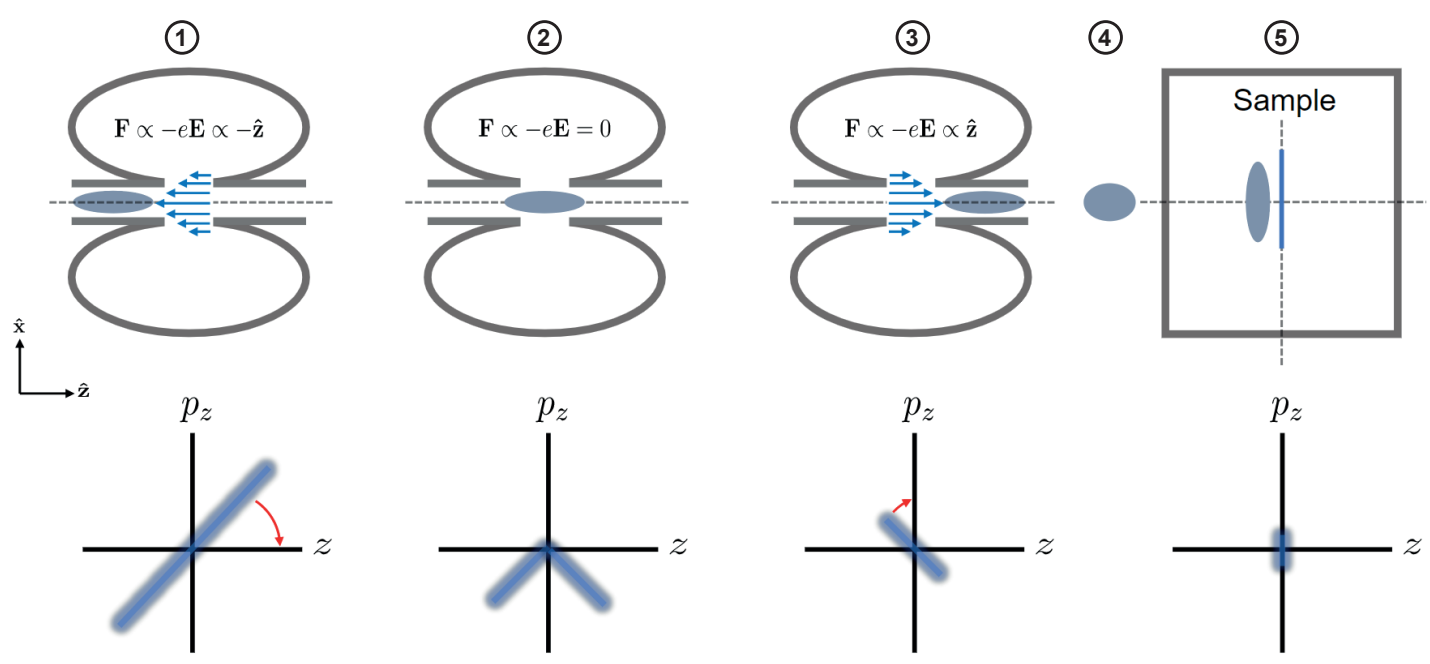

Figure 4.5: Radio-frequency electron pulse compression. A sequence of five states illustrates the momentary electron position and field (top row) as well as the phase space distribution (bottom row). It allows for temporal refocusing and, as a consequence, ultrashort electron pulses in the sample plane. Adapted from [260].

In a separate project, we pursue this alternative strategy as it may allow us to achieve low-energy electron pulses with subpicosecond duration when combined with the microgun. Additionally, a major advantage lies in an increased temporal focus length which would result in a larger gun-sample 
distance. This could strongly facilitate the handling during the experiment.

A third avenue, distinct from gun miniaturization and pulse compression, incorporates a concept from low-energy electron microscopy (LEEM). Here, the electron source provides electron energies in the keV-regime which are strongly decelerated in front of the sample down to electron energies of a few eV. In a setup with a pulsed electron source (see 4.6), this strategy is very beneficial since higher electron energies prevent the pulse from broadening. However, as Figure 4.6 suggests, this type of approach affects the entire geometry of the experiment. While an ultrafast implementation of LEEM would allow for time-resolved real-space imaging of surface dynamics, at this point, it is unclear whether the diffraction mode of such an instrument could match the momentum resolution of ULEED. Nonetheless, a spatial resolution of few $\mathrm{nm}$ in an ultrafast LEEM setup would enable nanodiffraction on a picosecond and potentially even femtosecond time scale. However, a detailed discussion of this new type of experiment goes beyond the scope of this section.

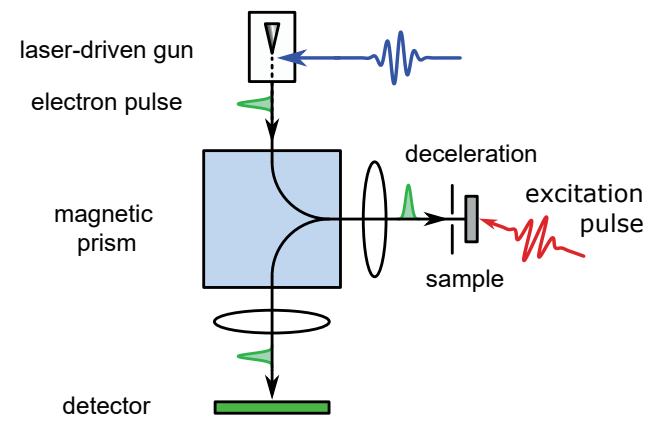

Figure 4.6: Sketch of a LEEM with a pulsed source.

\subsection{CDW-related Prospects}

The time-resolved study of the incommensurate phases of $1 T-\mathrm{TaS}_{2}$ with ps-resolution, presented in Chapter 3, highlighted the role of CDW-coupled excitations on the order parameter. Other aspects of CDW fluctuation modes require further investigation and will be briefly discussed.

Diffuse Scattering An interesting aspect concerns the question of diffuse scattering caused by phase fluctuations. According to Wang et al. [151], low-energy phasons scatter into the vicinity of satellite peaks and dominate the spot profile exhibiting a dependence $q^{-2}$ of the phason momentum. Based on this work, Minor et al. investigated spot profiles of $1 T-\mathrm{TaS}_{2}$ using x-ray diffraction in order to measure the phason thermal-diffuse scattering. In their measurement, they analyze satellites close to the main peaks (010) and (030) in the IC phase. Line profile fits provide phason velocities 
of propagation in all directions, showing a strong anisotropy that is expected for layered compounds. However, comparing a model with such a high number of fit parameters to the experiment in a meaningful way, requires high quality data, which is very challenging to achieve. This is necessary to reliably rule out other mechanisms affecting the line shape, e.g. CDW defects or non-CDW phonons. In the time-resolved study presented in this work, we observe a significant difference between the peak and integrated intensity of satellite reflexes for the highest fluence. We interpret this reshaping as the creation of CDW defects since low-energy phasons should be also observable for lower fluences. In order to observe a potential phason 'halo' very close to a satellite spot, we believe that a higher momentum resolution of the electron gun is necessary.

While Wang et al. [151] proposed an explicit expression for the line shape of a satellite reflex, equation 1.72 offers a more general approach to predict the impact of amplitude and phase fluctuations on the diffuse background. From a theoretical point of view, the main challenge for reliable predictions, however, lies in the accessability to the phonon population $n_{j, k}$, dispersion $\omega_{j, k}$ and polarization $\hat{\boldsymbol{e}}_{j, p, \boldsymbol{s}}$. In order to obtain the phonon dispersion and polarization of complex materials, it is usually necessary to use sophisticated computational techniques [176, 177, 234]. For CDW systems, this is exceedingly challenging because the coupling between electronic and vibrational degrees of freedom, the phonon softening and the incommensurablity have to be taken into account. In equilibrium, reasonable assumptions can be made for the phonon population $n_{j, k}$. However, in a non-equilibrium scenario, it is a difficult task to model the distribution of phonons because the number of populated phonons at different momenta in different branches changes over time (see also Fig. 1.21). In ultrafast experiments [261-263], a common way to predict the dynamics of phonon populations is given by the framework of multi-temperature models which, however, often simplify the problem by using equilibrium distributions as approximation.

Impact of Pinning Further investigation concerns the effect of pinning that breaks the translation invariance in incommensurate CDW systems and introduces a finite gap in the phason spectrum [71]. This effect has mostly been studied in x-ray experiments [264, 265] and electric transport measurements observing a nonlinear conductivity $[71,99,266]$. However, little is known about how pinning affects the ultrafast dynamics of collective modes in a diffraction pattern. Future experiments could therefore study the impact of impurity pinning, for example, in an intercalated system [265] or commensurability pinning in the commensurate phase of $1 T-\mathrm{TaS}_{2}$. A comparative study could reveal the influence of pinning on the relaxation dynamics of fluctuation modes. 
Exotic Phases $1 T-\mathrm{TaS}_{2}$ is known for its unique properties offering a wealth of phases and phase transitions. Stojchevska et al. reported about a 'hidden' state in $1 T-\mathrm{TaS}_{2}$ that emerges from the commensurate phase at very low temperatures and under intense femtosecond laser illumination [7]. In comparison to the other phases of the system, this non-equilibrium state exhibits a large drop of electrical resistance, strongly modified single-particle and collective-mode spectra, and a marked change of optical reflectivity. With the considerably improved temporal resolution of ULEED, great insight could been gained into the unique fluctuation modes of this exotic phase. It should be noted, however, that the measurement of the phase transition necessitates a modified pump-probe scheme (see Fig. 3.1) because of the state's long life time in the order of tens to hundreds seconds [232]. Solutions to this problem could be significantly increased repetition rates or intermediate 'erasing' pulses.

Furthermore, as has been shown by Vogelgesang et al. [14], the phase ordering kinetics of the NC-IC phase transition are governed by the dynamics of CDW defects. An interesting open question concerns the nature of the transient ensemble of CDW defects in the IC phase after the phase transition that potentially has characteristics of a so-called hexatic phase [267]. This phase has a specific order defined by characteristic translational and orientational correlations of the involved CDW defects. In $1 T-\mathrm{TaS}_{2}$, a hexatic phase has been artificially created via $\mathrm{Nb}$ impurity doping and was studied by means of scanning tunneling microscopy [268]. In diffraction, the CDW defect correlations of the hexatic phase should be directly observable in the radial and azimuthal broadening of satellite reflexes [269] (see Fig. B.1). Additionally, since CDW defects can be seen as CDW phase singularities, phase fluctuations are expected to play a relevant role which remains to be clarified.

Further Sample Systems Generally, a strength of the presented ULEED technique is its sensitivity to the first few layers of a material. While, in this work, the surface of the layered $1 T-\mathrm{TaS}_{2}$ bulk structure has been studied, including its complex interlayer couplings, the study of CDW dynamics in a single-layer should yield interesting complementary results. In a monolayer, the lack of interlayer coupling, strongly influences electron correlation [270,271] and affects the formation and properties of the charge-density wave. Along this line, interesting CDW sample systems comprise different types of TMDC monolayer and heterostructures [160, 271, 272], or quasi-1d charge-density waves on semiconductor and metal surfaces [43-45]. Recently, the ultrafast dynamics of self-assembled atomic indium chains on $\mathrm{Si}(111)$ have been studied in our group [48] featuring the coherent control over the metal-insulator structural phase transition in this system. Combined with the enhanced temporal resolution, developed in this work, more insight can be gained about the structural modes that drive this phase transition. 
Apart from the class of TMDCs, molybdenum bronzes [11, 37, 273] and tellurides [15, 38, 193, 274] have been subject to intense investigation, featuring a large set of different collective modes and highlighting the role of fluctuations in phase transtitions. Furthermore, CDWs in cuprates hosting high-temperature superconductivity constitute an interesting field of study [275]. The underlying mechanism for the emergence of both superconductivity and charge-density waves has a common origin in the coupling of electrons to the lattice $[273,276]$. The role of CDW fluctuations in cuprates has been crucial for the mutual relationship between these two effects and has not yet been full understood [277]. More specifically, it is still unclear how these fluctuation characteristics influence the superconducting state and if they compete or even enable it [275].

Interesting parallels could be drawn to other types of incommensurate systems. While CDWs exhibit displacive incommensurateness, intergrowth or overgrowth structures, such as rare-gas monolayers (Ar or $\mathrm{Kr}$ ) on graphite or $\mathrm{Hg}_{3-\delta} \mathrm{AsF}_{6}$, show incommensurateness between two different atomic lattices $[69,116]$. Specifically, rare-gas monolayers have been subject to extensive experimental as well as theoretical studies [278-288]. These systems are particularly known for phase transitions between commensurate and incommensurate phases, as well as melting transitions to fluid phases which occur only in low-dimensional systems [80].

\subsection{Concluding Remarks}

In summary, this work includes important contributions in the field of ultrafast dynamics and surface science. The development of a new ultrafast electron gun has significantly shifted the limits of the achievable temporal resolutions of the ULEED technique and considerably extended the class of observable dynamic processes. In addition to the technological progress, new insights into the structural dynamics of a broken-symmetry state could be gained by investigating the prototypical CDW system $1 T-\mathrm{TaS}_{2}$. In conclusion, ULEED paves the way to the investigation of an incredible wealth of previously inaccessible dynamics and represents a versatile tool to study systems in reduced dimensions, such as layered materials and adsorbed monolayers. 


\section{A Symbols and Abbreviations}

$\alpha$

$\gamma$

$\Delta$

$\delta$

$\epsilon$

$\epsilon_{0}$

$\eta$

$\theta$

$\theta_{D}$

$\lambda$

$\mu$

$\xi$

$\rho$

$\sigma$

$\tau$

$\Phi$

$\phi$

$\varphi$

$\chi$

$\psi$

$\omega$

$\AA$

A

$\boldsymbol{A}$

angle

reciprocal domain size

complex order parameter, electronic gap

amplitude fluctuation, Kronecker delta

energy of a state

dielectric constant

renormalized amplitude

angle, theta function

Debye temperature

wave length, dimensionless electron-phonon coupling constant

chemical potential, atomic mass

integer

charge density

width of distribution

time constant

pair correlation function

potential, angle, complex amplitude

phase of complex order parameter, phase fluctuation

static response or susceptibility

order parameter

frequency

Ångström

scattering amplitude

PLD amplitude 


\begin{tabular}{|c|c|}
\hline$a$ & lattice constant or vector \\
\hline$a^{*}$ & reciprocal lattice constant or vector \\
\hline$b, b^{+}$ & bosonic ladder operators \\
\hline $\boldsymbol{b}$ & superstructure lattice vector, Burgers vector \\
\hline$C$ & constant \\
\hline$c, c^{+}$ & fermionic ladder operators, constant \\
\hline$E$ & energy \\
\hline$E_{k}$ & single electron dispersion \\
\hline$e$ & electron charge \\
\hline$\hat{\boldsymbol{e}}$ & phonon polarization vector \\
\hline$F$ & free-energy, structure factor, fluence \\
\hline$f$ & Fermi function, atomic form factor \\
\hline$G$ & reciprocal vector, lattice factor \\
\hline$g$ & electron-phonon coupling constant \\
\hline$g$ & lattice vector \\
\hline$H$ & Hamiltonian \\
\hline$\hbar$ & reduced Planck constant \\
\hline$I$ & intensity \\
\hline$i$ & imaginary unit \\
\hline$J$ & intensity, Bessel function \\
\hline $\boldsymbol{k}$ & wave vector \\
\hline $\boldsymbol{k}_{F}$ & Fermi wave vector \\
\hline$k_{B}$ & Boltzmann constant \\
\hline$L$ & equilibrium lattice site \\
\hline$M$ & mass of atom, Debye-Waller exponent \\
\hline$m$ & mass, integer \\
\hline$m^{*}$ & effective mass \\
\hline$N$ & number density of atoms, integer \\
\hline$n$ & electron density, integer, defect density, population \\
\hline$O$ & higher orders in expansion \\
\hline$p$ & number of conduction electrons per atom \\
\hline$Q$ & wave vector \\
\hline$q$ & wave vector \\
\hline$\delta q$ & wave vector \\
\hline
\end{tabular}




$\begin{array}{ll}\boldsymbol{R} & \text { postion vector of unit cell } \\ \boldsymbol{r} & \text { position vector } \\ \boldsymbol{s} & \text { scattering vector } \\ T & \text { temperature, instrumental response function } \\ t & \text { transfer function, time } \\ U & \text { Coulomb energy, atomic potential } \\ u & \text { lattice displacement } \\ V & \text { atomic potential, exchange energy } \\ v & \text { effective potential } \\ v_{F} & \text { Fermi velocity } \\ W & \text { Debye-Waller exponent } \\ x & \text { position in space } \\ z & \text { position }\end{array}$

$\begin{array}{ll}\text { ARPES } & \text { angle-resolved photoemission spectroscopy } \\ \text { BCPS } & \text { molecular compound }\left(\mathrm{ClC}_{6} \mathrm{D}_{4}\right)_{2} \mathrm{SO}_{2} \\ \text { C } & \text { commensurate } \\ \text { CCD } & \text { charge-coupled device } \\ \text { CDW } & \text { charge-density wave } \\ \text { CMOS } & \text { complementary metal oxide semiconductor } \\ \text { ERC } & \text { european research council } \\ \text { eV } & \text { electron volt } \\ \text { FEM } & \text { finite element modeling } \\ \text { FIB } & \text { focused-ion-beam } \\ \text { fs } & \text { femtosecond } \\ \text { FWHM } & \text { full-width at half-maximum } \\ \text { GAUSS } & \text { Georg-August University School of Science } \\ \text { GDA } & \text { Gaussian displacement approximation } \\ \text { GPA } & \text { Gaussian phase approximation } \\ \text { IC } & \text { incommensurate } \\ \text { LEED } & \text { low-energy electron diffraction } \\ \text { LEEM } & \text { low-energy electron microscopy } \\ \text { mbar } & \text { millibar } \\ \text { mm } & \text { millimeter }\end{array}$




$\begin{array}{ll}\mu \mathrm{m} & \text { micrometer } \\ \mathrm{MCP} & \text { multi-channel plate } \\ \mathrm{NC} & \text { nearly commensurate } \\ \mathrm{nm} & \text { nanometer } \\ \mathrm{NMR} & \text { nuclear magnetic resonance } \\ \text { NOPA } & \text { noncollinear optical parametric amplifier } \\ \text { OPA } & \text { optical parametric amplifier } \\ \text { PLD } & \text { periodic lattice displacement } \\ \text { ps } & \text { picosecond } \\ \text { RHEED } & \text { reflection high-energy electron diffraction } \\ \text { rms } & \text { root-mean-square } \\ \text { TEM } & \text { transmission electron microscopy } \\ \text { TMDC } & \text { transition metal dichalcogenide } \\ \text { TTF-TCNQ } & \text { tetrathiafulvalene-tetracyanoquinodimethane } \\ \text { UHV } & \text { ultra-high vacuum } \\ \text { ULEED } & \text { ultrafast low-energy electron diffraction } \\ \text { UTEM } & \text { ultrafast transmission electron microscopy }\end{array}$




\section{B | NC-IC Spot Broadening}

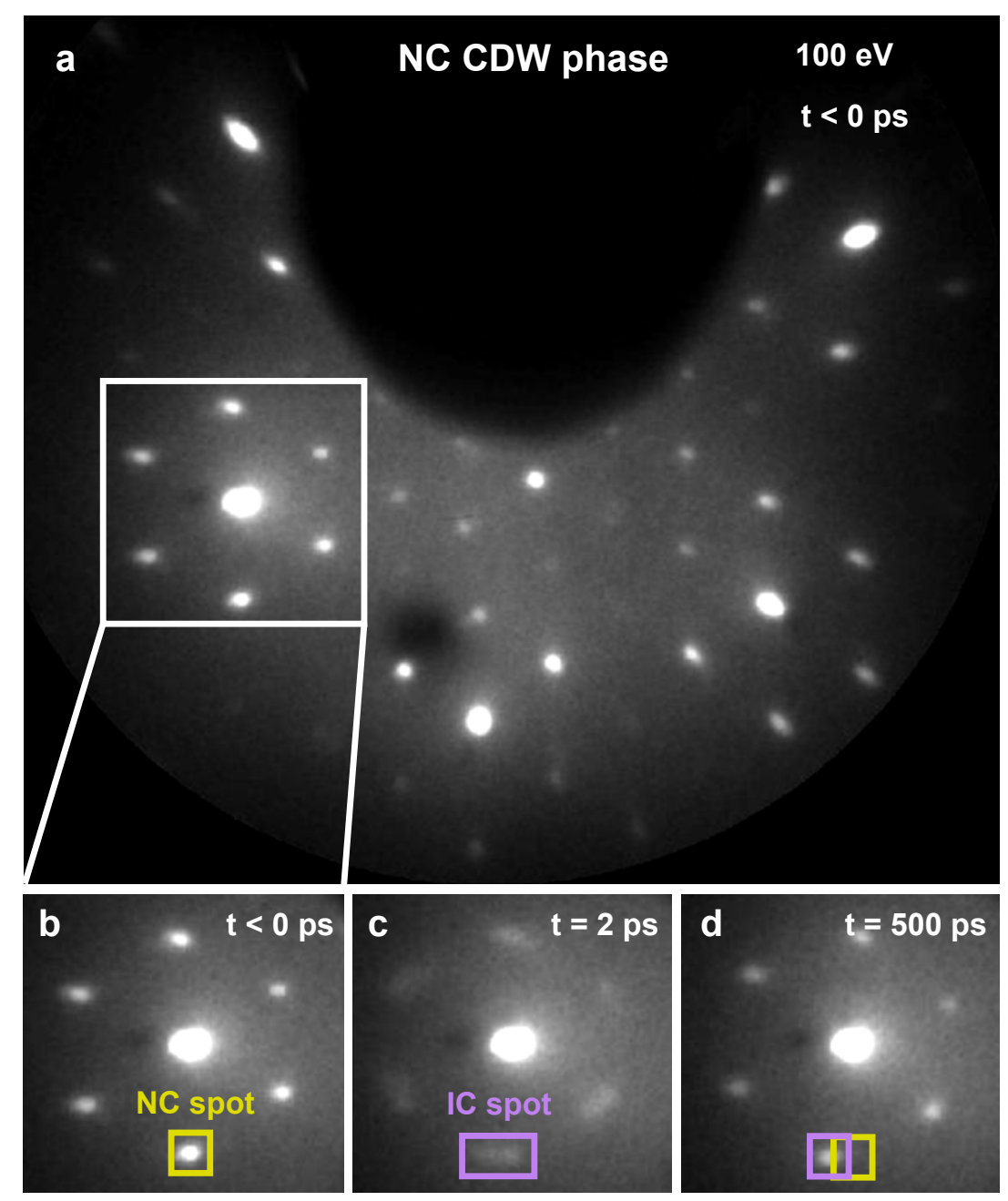

Figure B.1: Preliminary data on the ultrafast NC-IC phase transition $1 T-\mathrm{TaS}_{2}$. (a) Diffraction pattern of $\mathrm{NC}$ phase for negative delay at room temperature. (b)-(d) Time frames for a fluence of $F=2.7 \mathrm{~mJ} / \mathrm{cm}^{2}$, including a single main and six first-order satellite spots. During the phase transition, the intensity of NC spots rapidly decreases paralleled by the emergence of azimuthally broadened IC satellite spots. The position and approximate size of NC (yellow) and IC (violet) reflexes are indicated by rectangles. 


\section{C | FEM of Sup-Ext-Unit}

Figure C.1 shows maps of transmission, pulse duration and normalized emittance for varying suppressor and extractor voltage. The electron trajectories were obtained by the same method, discussed in Chapter 2. All three maps exhibit a common feature in the bottom left corner. This feature defines a diagonal line (violet line), refered to as cut-off line, below of which no or few particles are transmitted and, accordingly, the pulse width and the emittance are not defined. More specifically, in the triangular shaped area, the electric field around the apex of the nanotip (see $2.2 \mathrm{~b}$ ) flips sign and electrons are accelerated back to the tip's surface after emission (see Fig. C.1d). Accordingly, parallel lines correspond to different sign-change-positions, either being in front of the tip's apex or behind. Moving along a line in the map, the strength of the extraction field changes, with stronger fields towards the top left.

As shown in Fig. C.1a, transmission values are high $(55-65 \%)$ close to the cut-off line, while decreasing with distance. The transmission percentage refers to the total number of calculated electrons per voltage pair (around 50k). For the applied voltage intervals, the overall pulse duration lies at 1 ps or below. The smallest values of around $200 \mathrm{fs}$ can be achieved near the cut-off line. However, directly on the line, the pulse width strongly increases to values around $400 \mathrm{fs}$ because some particles are already subjected to a decelerating field (see Fig. C.1d) which broadens the position distribution along the gun axis.

The emittance is a useful quantity for describing the quality of electrons beams [289]. The normalized root-mean-square (rms) emittance is defined as

$$
\tilde{\epsilon_{n}}=\frac{v_{z} / c}{\sqrt{1-\left(v_{z} / c\right)^{2}}} \sqrt{\left\langle x^{2}\right\rangle\left\langle x^{\prime 2}\right\rangle-\left\langle x x^{\prime}\right\rangle^{2}}
$$

where $v_{z}$ is the electron velocity in along the gun axis, $c$ is the speed of light, $\left\langle x^{2}\right\rangle,\left\langle x^{\prime 2}\right\rangle$ and $\left\langle x x^{\prime}\right\rangle^{2}$ are the moments of the electron distribution in trace space, a plane perpendicular to the beam propagation [289]. The two first terms denote the standard deviations of position and slope, whereas the third measures the correlations between the first two.

For the given set of parameters, the normalized rms emittance (Fig. C.1c) of the microgun lies 
in the range of about 100-350 $\mathrm{nm}$ mrad. This compares well to the experimental value of about $250 \mathrm{~nm}$ rad (see Chapter 2). The emittance varies strongly in close vicinity to the cut-off line, whereas, for all other voltage pairs, it shows a rather weak dependence. The behavior near the cut-off line is connected to the interplay between the sup-ext-unit and the following Einzel lens. A strong collimation right behind the tip apex (Fig. C.1d) reduces spherical aberration of the following Einzel lens since trajectories enter the lens close to the symmetry axis. For a more divergent beam, particles distant from the axis suffer more significantly from aberration or are cut off by an electrode, setting an upper limit for the emittance.
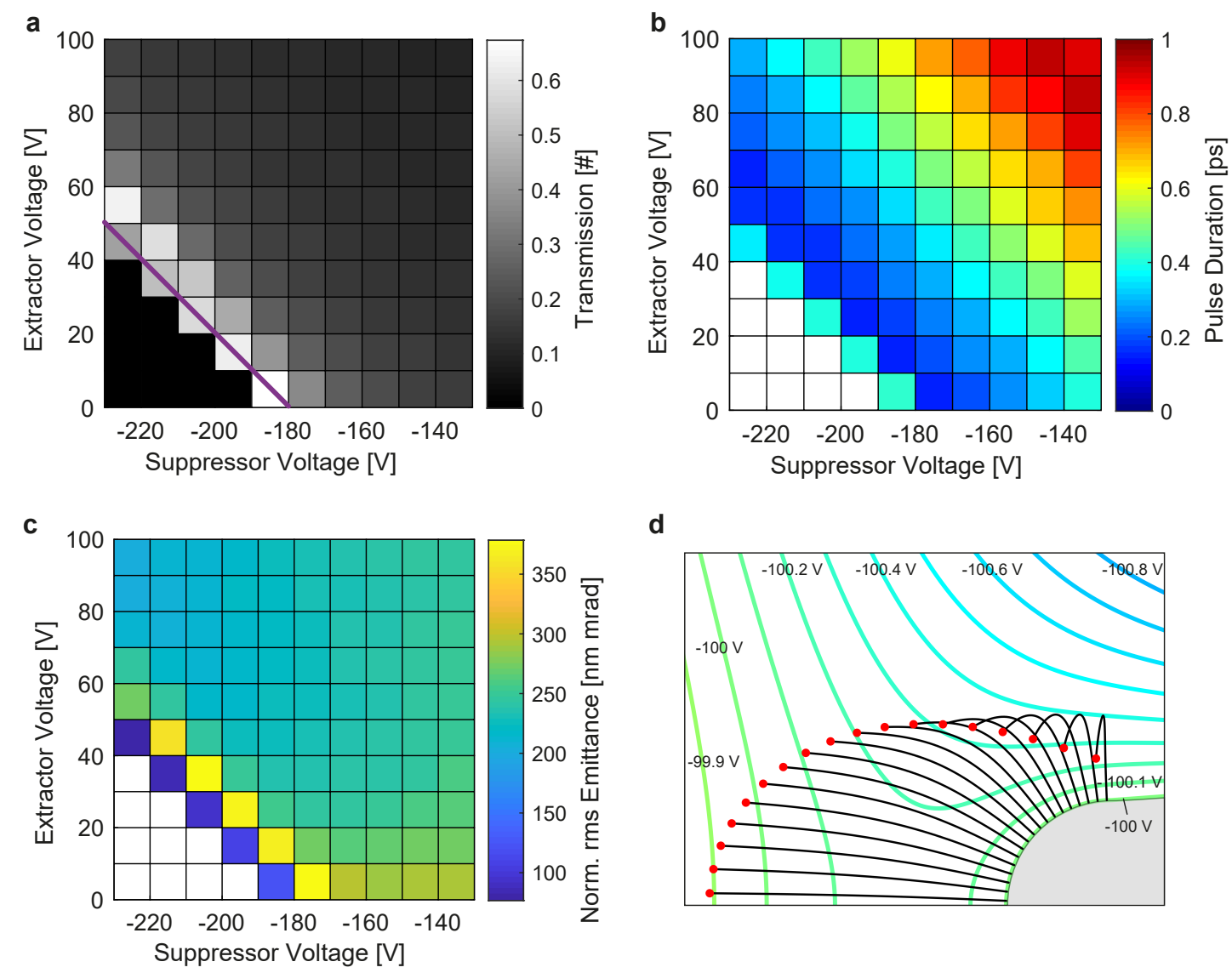

d

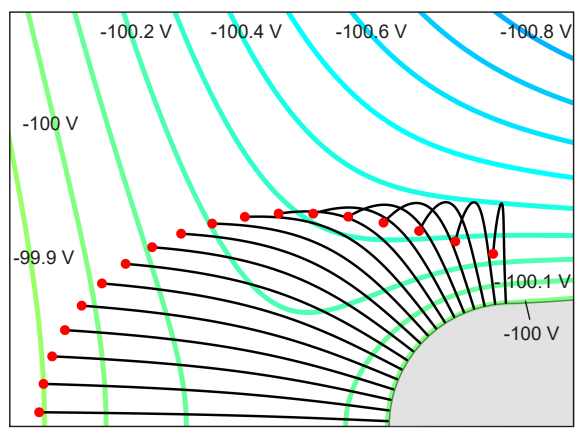

Figure C.1: Electron gun modeling. Maps of (a) the transmission, (b) the pulse duration and (c) the normalized radial emittance in dependence of suppressor and extractor voltage for an electron energy of $100 \mathrm{eV}$ and fixed lens voltage of $-180 \mathrm{~V}$. All values are obtained in a plane $400 \mu \mathrm{m}$ behind the ground electrode. For each voltage pair, a number of 51480 trajectories were calculated. Transmission values lower than 4000 particles were set to zero. The initial kinetic energy width was chosen to be $\sigma_{E}=0.5 \mathrm{eV}$. (d) Trajectories (black lines) and local electric field for a voltage setting of $(-100|-190| 0 \mid 180)$ V. Electrons emitted on the side of the apex are strongly decelerated and bent towards the symmetry axis. 


\section{D $\mid$ Electron Gun Holder}
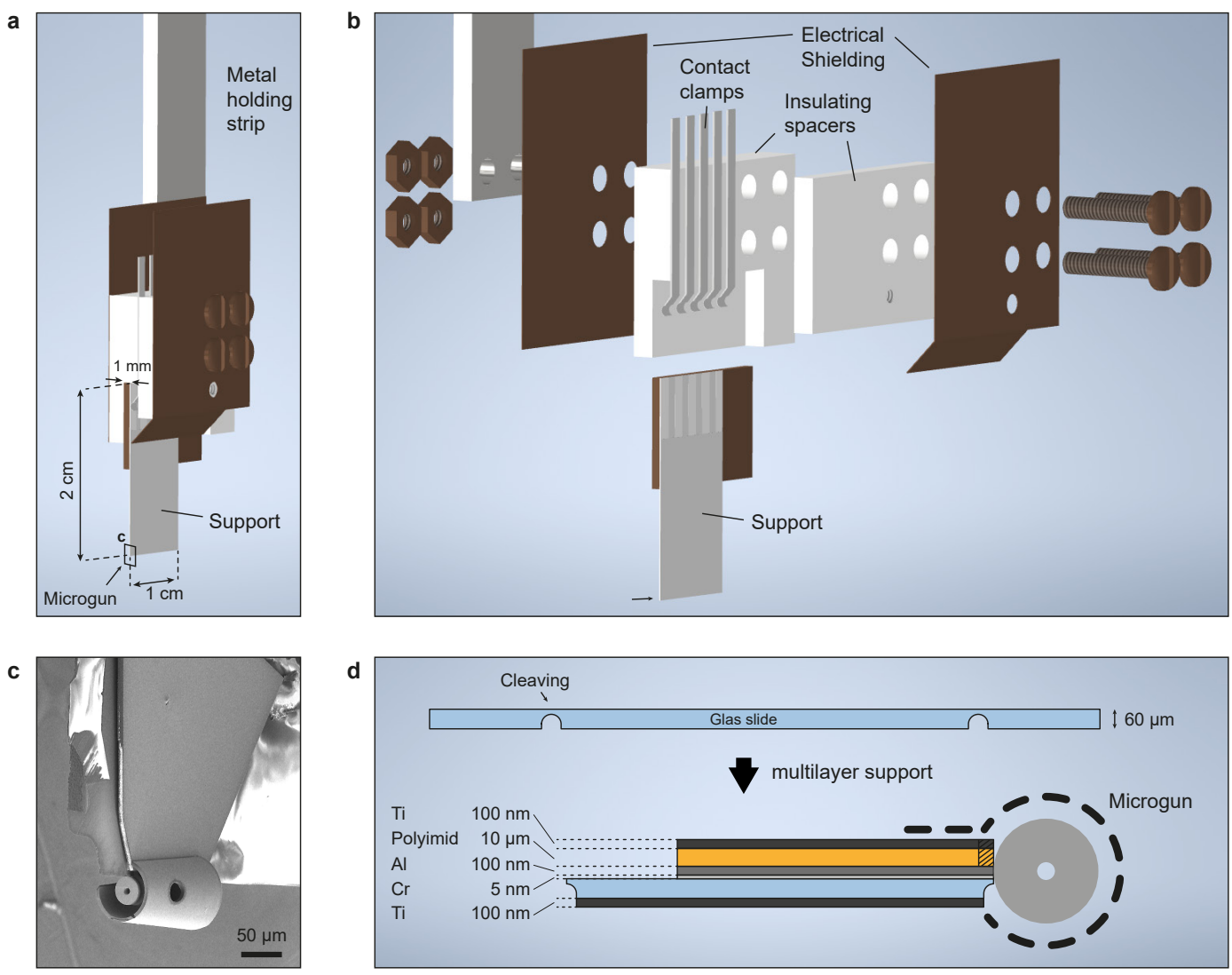

Figure D.1: Technical overview. (a) Sketch of gun holder design. The holder is attached to a metal holding strip that is mounted at the top of the vacuum chamber. The microgun support is inserted into the holder from below where it is electrically contacted and held in position by metal clamps. (b) Exploded view of holder showing individual components. The used materials include stainless steel, bronze and polytetrafluorethylen (Teflon). (c) Micrograph of shielded microgun. White patches indicate charged areas by the electron beam. (d) Sketch of support fabrication. Starting from a thin glass support, a multilayer stack is fabricated using photolithographic techniques. It comprises metal strip lines for voltage contacts as well as the electrical shielding. 


\section{E | Diffraction Image Processing}

This section describes the structure of measured data sets and provides an insight into the image postprocessing procedures including the correction of distortions in the recorded diffraction patterns.

\section{Structure of measured data}

In the context of this work, a run of the ULEED experiment is characterized by a number of parameters, such as the specific CDW phase $\{\mathrm{IC}, \mathrm{NC}\}$, the electron energy $\{80 \mathrm{eV}, 100 \mathrm{eV}\}$ and the pump fluence $\left\{\mathrm{F}_{1}, \mathrm{~F}_{2}, \mathrm{~F}_{3}\right\}$. This results in a large collection of run data sets because almost all parameter combinations were realized. Each measurement run consists of several loops. A single loop is a stack of diffraction images in which one image corresponds to a given time delay (see Fig. E.1). The measurement duration for a single loop is approximately one hour leading to a total run time of several hours. In order to improve the signal-to-noise ratio, loops within a measurement run

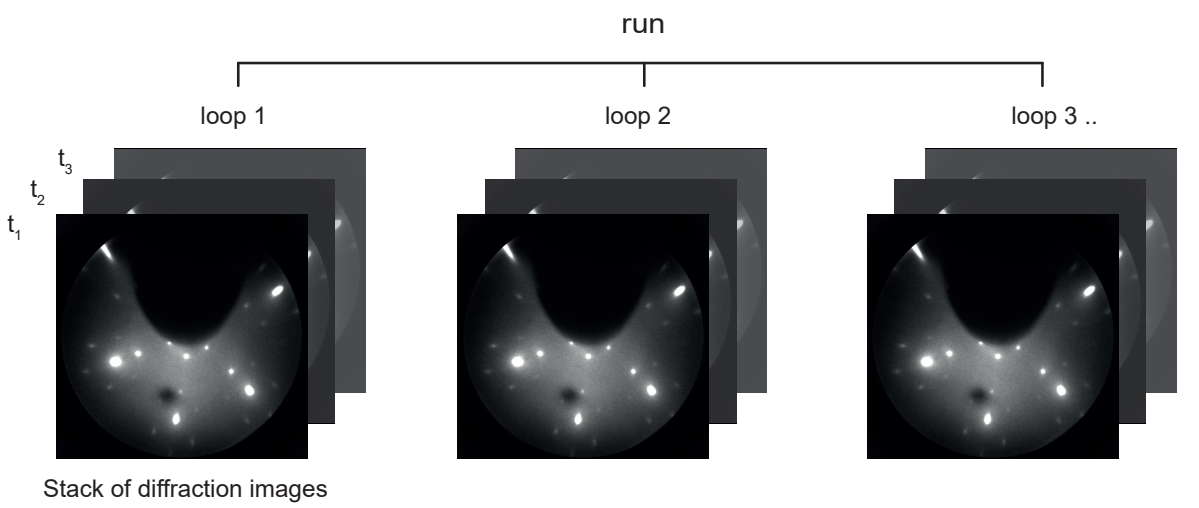

Figure E.1: Structure of a data set. Measurement run (IC-CDW of $1 T-\mathrm{TaS}_{2}$ at an electron energy of $80 \mathrm{eV}$ for a fixed fluence) containing three loops. A loop consists of a stack of diffraction images in which each frame corresponds to a specific time delay $\left\{\mathrm{t}_{1}, \mathrm{t}_{2}, \mathrm{t}_{3} \ldots\right\}$.

are merged into a single one using the following procedure. In a first step, a median filter applied to each individual diffraction image lowers the noise as it removes pixels with very large or low values (hot and dead pixels). Secondly, at a fixed time delay, diffraction patterns for loops $n>1$ are 
compared with the diffraction pattern of the first loop and corrected for small shifts that occur due to charging effects of the electron gun or sample drift. These shifts are usually small between two neighboring loops, but larger between loops that are temporally further apart. Thirdly, for a given time delay, diffraction images of all loops are averaged with weights given by the summed total intensity of each individual image. This collapses all loops to a single stack of diffraction images.

\section{Correction of image distortions}

In general, diffraction techniques provide access to the reciprocal lattice of a crystal structure by projecting the diffracted far field onto a detector. In LEED, the radius of curvature of the associated Ewald sphere is small which typically results in large scattering angles for low-order beams. Commonly, a detector shaped as a spherical cap facilitates the detection of the diffracted beams and allows to directly map the Ewald sphere. The diffracted image on the sphere is then projected onto a flat detector using a suitable camera system (see Fig. E.2a). Any deviation from this idealized scheme leads to geometric distortions in the final image (see Fig. E.2b-d). In our experimental setup, geometric distortions are introduced by a planar electron detector, non-normal beam incidence on the sample and inadequate alignment of detector and sample plane. A second source of distortions enters through electromagnetic fields which alter the electron's trajectory. These fields include a weak decelerating electrostatic field at the front plate of the MCP to filter out electrons with an energy lower than approximately $15 \mathrm{eV}$, and a rather inhomogeneous magnetostatic field introduced through a strong magnet attached at the side of the UHV chamber in order to control the position of the diffraction pattern on the planar detector. The combination of geometrical and field distortions (see Fig. E.3b or E.4c) strongly complicates the correction process.

In the following, a number of approaches for image correction are briefly presented. All but the last one of these methods are based on the minimization of a cost function $U$ that varies with predefined length given by reflex positions and prior knowledge of the materials system. A global minimization algorithm (MATLAB: Simulanneal, GobalSearch) searches for the global minimum in the high dimensional landscape of $U$ to find the optimal parameters of a correction function that removes the distortions in the image.

Polynomials In the first approach, the correction function is given by a radially symmetric polynomial

$$
f\left(\boldsymbol{r}_{s}\right)=\left(\boldsymbol{r}_{s}-\boldsymbol{r}_{c}\right)+a\left(\boldsymbol{r}_{s}-\boldsymbol{r}_{c}\right)^{2}+b\left(\boldsymbol{r}_{s}-\boldsymbol{r}_{c}\right)^{3}+c\left(\boldsymbol{r}_{s}-\boldsymbol{r}_{c}\right)^{4}+d\left(\boldsymbol{r}_{s}-\boldsymbol{r}_{c}\right)^{5} \ldots
$$


where the center of distortion is given by $\boldsymbol{r}_{c}$ and the vector $\boldsymbol{r}_{s}$ denotes a point in the image, e.g. a diffraction reflex. In optics, these simplistic polynomials are often used to correct for barrel and pincushion distortions. The parameters $a, b, c$ and $d$ are determined by minimizing the cost function

$$
U=\sum_{i}\left(\left(\frac{d_{m, i}}{d_{s a t, i}}-f_{\text {rat }}\right)^{2}+\left(\bar{d}_{m}-d_{m, i}\right)^{2}+\left(\bar{d}_{s a t}-d_{\text {sat }, i}\right)^{2}\right) .
$$

The quantities $d_{m, i}$ and $d_{s a t, i}$ denote the distances between selected main and satellite diffraction reflexes, respectively, and $\bar{d}_{m}$ and $\bar{d}_{s a t}$ their average values. The constant $f_{\text {rat }}$ is the known ratio between main lattice and CDW periodicity. The cost function $U$ approaches its minimum when the selected distances approach their average values and their ratio of the distances approaches the constant $f_{\text {rat }}$.

Although this method partially corrects geometric distortions in the diffraction pattern, it is not suitable for including non-radial and very localized distortions in the image. Moreover, to correct for larger distortions at the edge of the image an increasing number of polynomial orders has to be included. An alternative approach that allows to extend the set of representable functions, is to decompose the distortion field into gradients of Zernike polynomials $Z_{n}^{m}(\rho, \varphi)$ which are often used in optics and imaging [290]. However, in order to properly represent a complicated, non-radial distortion field, high-order Zernike polynomials have to be taken into account. This results in strong divergences at the edges of the distortion field which are challenging to control.

Gnomonic projection The next approach considers the actual geometry of the backscattering diffraction experiment, which consists of the conically diffracted beam from the sample and the projection onto a planar detector. In the field of map projections [291], this type of projection is called gnomonic (see Fig. E.2b-c). This projection leads to strong distortions at the edges of the image because points on the sphere with high latitude are projected far from the center of distortion in the detector plane. If the north pole and the tangent point do not coincide the additional tilt results in a non-radial distortion field (see Fig. E.2c). The coordinates of the tangent point and the radius of the sphere determine the distortion field. These parameters are obtained in a similar way minimizing the above mentioned cost function $U$. More specifically, selected spots in the diffraction pattern (Fig. E.2e) are back-projected onto a sphere (Fig. E.2f) according to a gnomonic map. New spot coordinates (red dots) are obtained by the orthographic view onto the north pole (Fig. E.2g). The minimization algorithm determines the optimal gnomonic projection and leads to a corrected diffraction spot coordinates shown in Figure E.2h.

In an extended version of this approach, a decelerating homogeneous electric field is taken into account which leads to parabolic electron trajectories (see Fig. E.2d). This alters the projected 
positions on the sphere. To determine the new positions, the emission angles in the sample plane are determined analytically for each diffraction spot, which are then used to guide the particles straight onto the sphere according to the angles obtained. The final new spot coordinates follow then from the intersection with the sphere and the orthographic projection facing the north pole. This method leads to additional minor corrections compared to the previous version.

Although both methods lead to a overall slightly less distorted diffraction pattern, some areas, especially in the upper half of the image, still show significant deviations (see Fig. E.2h).
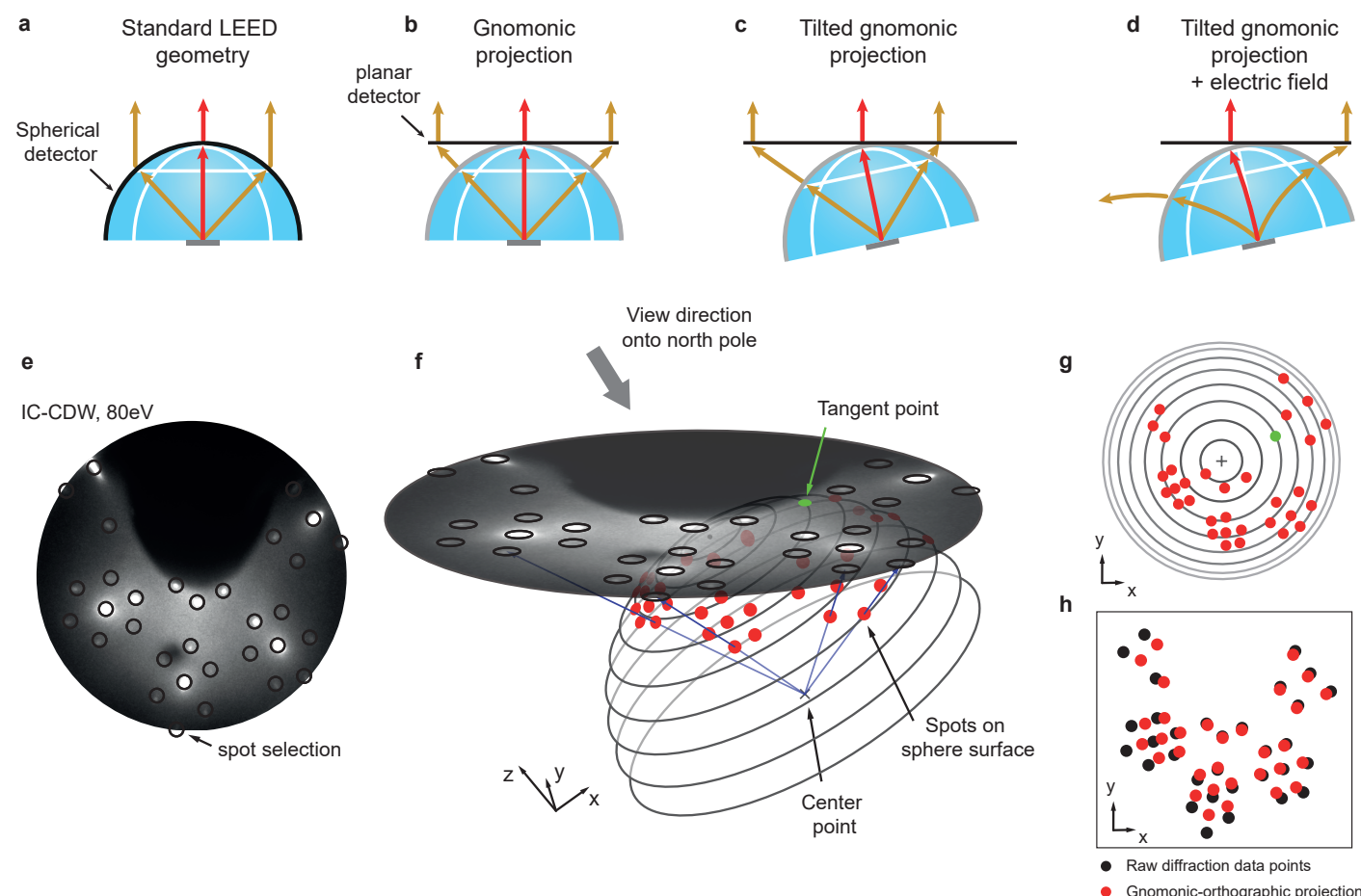

Figure E.2: Distortions caused by gnomonic projections. (a) Standard LEED geometry including a hemispherical electron detector. (b) A planar detector leads to a gnomonic distortion of the diffraction image. (c) An additional tilt of the emission cone results in a non-radial distortion. (d) A decelerating electric field leads to parabolic electron trajectories adding further distortion to the image. (e) Distorted diffraction pattern of the IC phase of $1 T-\mathrm{TaS}_{2}$ at an electron energy of $80 \mathrm{eV}$. Black circles indicate the positions of diffraction reflexes that are used in the correction procedure. (f) Sketch of the gnomonic map showing the diffraction pattern in the detector plane and electron trajectories (violet straight lines) that project coordinates on a half sphere (red dots). The illustrated gnomonic projection in (f) and (g) is an example and does not correspond to the optimal one determined by the algorithm (shown in (h)). (g) View on the north pole of the half sphere shown in (f). The orthographic projection yields the new set of corrected reflexes positions (red dots). (h) Comparison of diffraction spot positions obtained from the measurement (black dots) with the new positions given by the optimal gnomonic projection (red dots). 
a

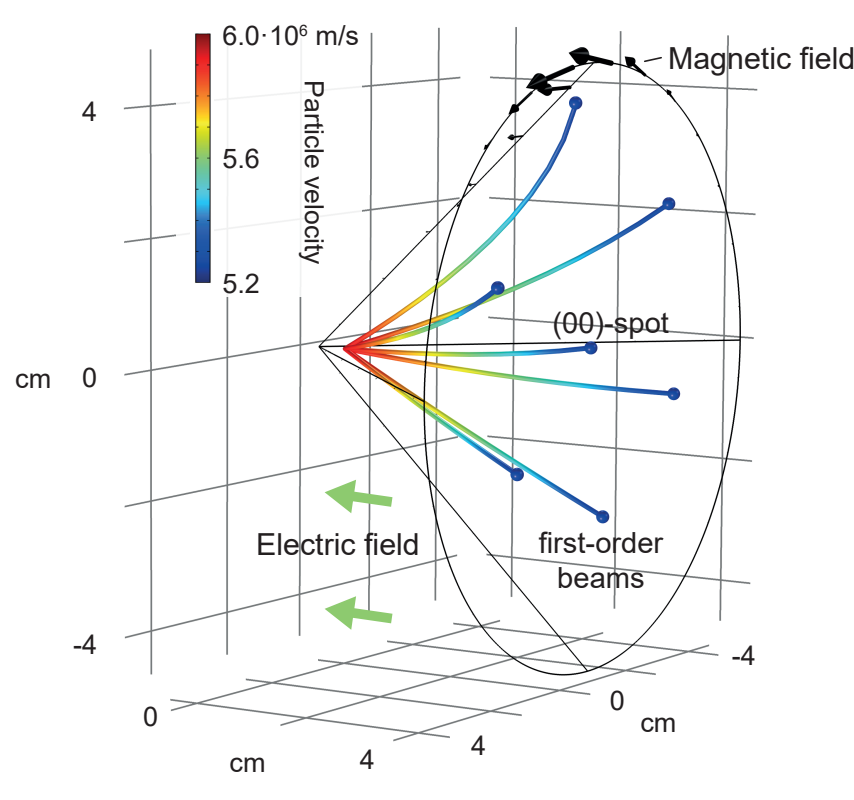

b

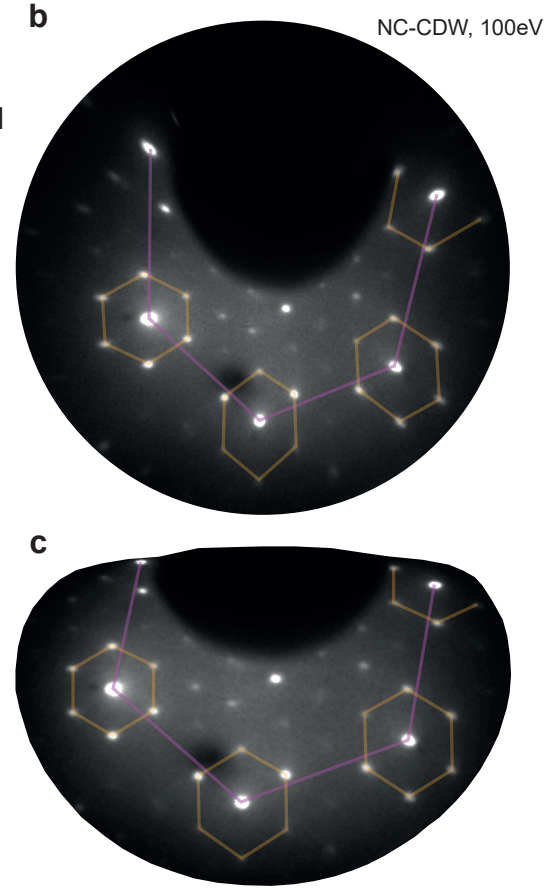

Figure E.3: Image correction via FEM. (a) Traces of particles propagated in an electromagnetic field using FEM. The color scale indicates the particle's velocity. The black and green arrows denote the magnetic and electric field, respectively. (b) Distorted diffraction pattern of the NC-phase of $1 T-\mathrm{TaS}_{2}$ at an electron energy of $100 \mathrm{eV}$. Colored hexaga serve as guide for the eye. (c) Undistorted image using the correction map obtained from the FEM model.

Image correction via FEM In a more comprehensive model, the geometry of the experiment as well as the decelerating electric field and the inhomogeneous magnetic field are considered. As illustrated in Fig. E.3a, particles (zero beam and first-order main lattice) are conically emitted and propagate in an electromagnetic field towards a planar detector using finite element modeling $\left(\mathrm{Comsol}^{\circledR}\right)$. The final positions on the detector are compared to the ones obtained from measurement yielding a specific cost function. Assuming a hexagonal diffraction pattern (see Fig. E.3b), the first-order main lattice beams are emitted each separated by an angle of $60^{\circ}$ in the plane. The free parameters in this model are the field strength of the magnetic dipole field, the opening angle of the emission cone, the two tilt angles of the cone, the azimuthal rotation angle of the first-order beams and the $\mathrm{x}$ - and $\mathrm{y}$-position of the center of the diffraction pattern. These optimal parameters are again obtained by minimizing the associated cost function. In the corrected image (see Fig. E.3c), it is apparent that the magnetic dipole field leads to a the strong vertical distortion of the diffraction pattern.

Although the present method covers many features of actual experimental setup, it is important 
to note that deviations from the real field distributions are challenging to include and complicate the full removal of all distortions in the diffraction image. More specifically, inhomogeneities in the homogeneous electric field and the magnetic dipole field as well as local electric fields around the electron gun are challenging to consider in this model.
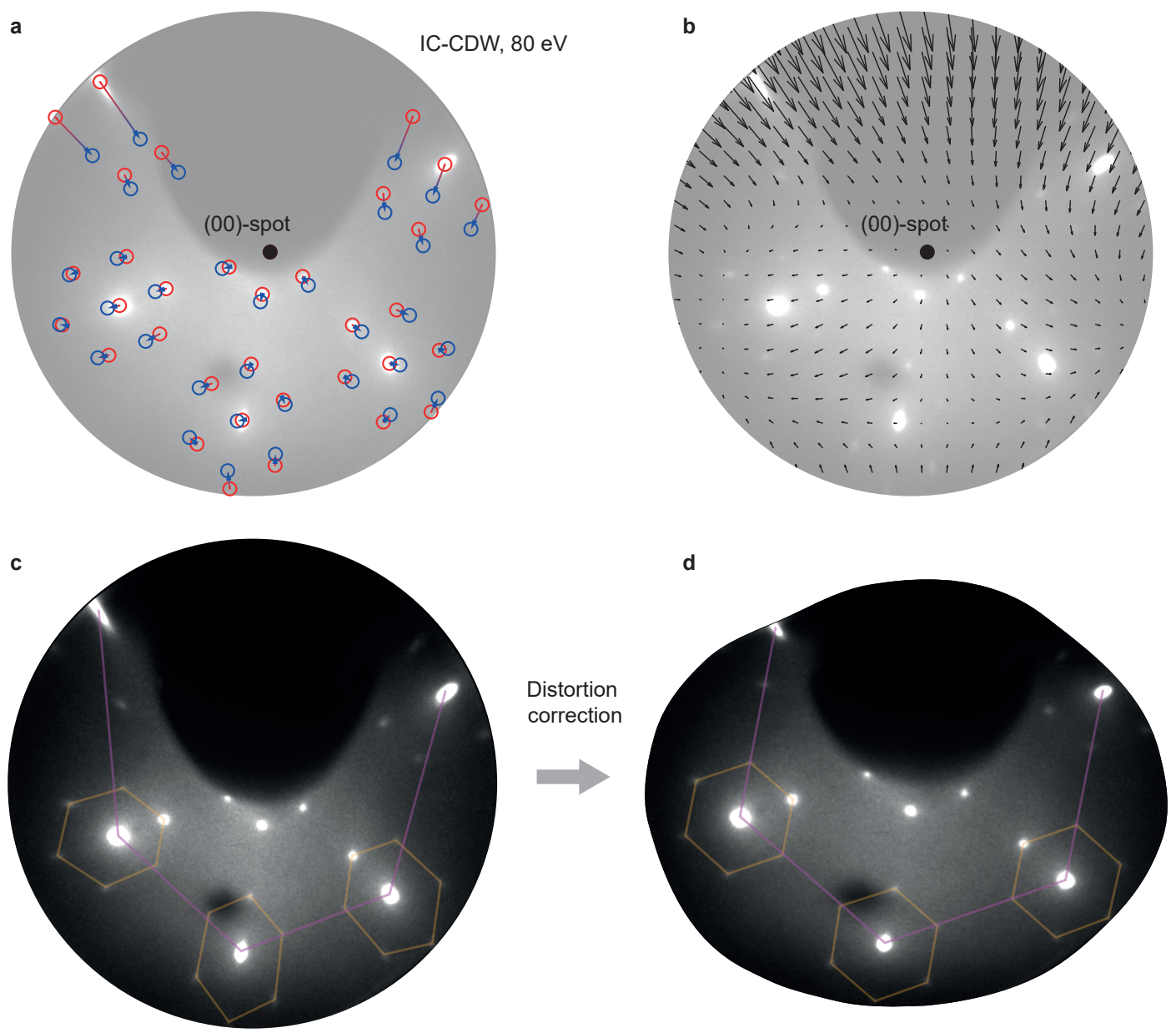

Figure E.4: Distortion correction via an interpolated vector field. (a) Diffraction pattern of the IC-phase of $1 T-\mathrm{TaS}_{2}$ at an electron energy of $80 \mathrm{eV}$. Blue circles indicate the positions of measured diffraction reflexes, while red circles denote the exact spot positions given by the crystal structure. (b) Interpolated vector field using vectors obtained in (a). (c) Distorted diffraction pattern. Colored hexaga serve as guide for the eye. (d) Corrected image obtained by applying vector field given in (b).

Interpolation of correction vector field In the previous approaches, a minimization algorithm was applied to find the optimal correction function within a specific class of parameter-dependent functions. In this last method, the correction map is obtained from the full knowledge of the crystal structure and the positions of the diffraction spots determined by the measurement. To this end, 
diffraction reflexes are selected in a measured diffraction image (see Fig. E.4a, red dots) that are then moved to the positions of the known structure (Fig. E.4b, blue dots). The full correction vector field (Fig. E.4c) that maps the intensities of all intermediate coordinates in the diffraction image to their new positions, is obtained via interpolation using biharmonic splines (MATLAB ${ }^{\circledR} 4$ griddata method). The application of the correction vector field on the distorted diffraction image (Fig.E.4c) leads then to the final undistorted image shown in Fig. E.4d.

The grade of the corrected image depends on the number of given diffraction reflexes in the image that can serve as support points in the interpolation algorithm. In comparison to the previous methods, it is important to point out that the correction via an interpolated vector field is only applicable if the crystal structure of the material has been fully resolved. Since the crystal structures of all CDW phases of $1 T-\mathrm{TaS}_{2}$ are known, the interpolation method was used troughout this work to correct the record the measured diffraction images. 


\section{Bibliography}

[1] W. E. King, G. H. Campbell, A. Frank, B. Reed, J. F. Schmerge, B. J. Siwick, B. C. Stuart, and P. M. Weber. Ultrafast electron microscopy in materials science, biology, and chemistry. Journal of Applied Physics, 97(11):111101, 2005. Cited on page 1.

[2] A. H. Zewail. Four-dimensional electron microscopy. Science, 328(5975):187-193, 2010. Cited on pages 1 and 55 .

[3] A. Rousse, C. Rischel, S. Fourmaux, I. Uschmann, S. Sebban, G. Grillon, P. Balcou, E. Förster, J. Geindre, P. Audebert, J. Gauthier, and D. Hulin. Non-thermal melting in semiconductors measured at femtosecond resolution. Nature, 410(6824):65-68, 2001. Cited on page 1.

[4] K. Sokolowski-Tinten, C. Blome, C. Dietrich, A. Tarasevitch, M. Horn von Hoegen, D. von der Linde, A. Cavalleri, J. Squier, and M. Kammler. Femtosecond X-Ray Measurement of Ultrafast Melting and Large Acoustic Transients. Physical Review Letters, 87(22):225701, 7, 2001. Cited on page 1.

[5] B. J. Siwick, J. R. Dwyer, R. E. Jordan, and R. J. D. Miller. An Atomic-Level View of Melting Using Femtosecond Electron Diffraction. Science, 302(5649):1382-1385, 21, 2003. Cited on pages 1,2 and 55 .

[6] A. Tomeljak, H. Schäfer, D. Städter, M. Beyer, K. Biljakovic, and J. Demsar. Dynamics of Photoinduced Charge-Density-Wave to Metal Phase Transition in $\mathrm{K}_{0.3} \mathrm{MoO}_{3}$. Physical Review Letters, 102(6), 13, 2009. Cited on page 1.

[7] L. Stojchevska, I. Vaskivskyi, T. Mertelj, P. Kusar, D. Svetin, S. Brazovskii, and D. Mihailovic. Ultrafast Switching to a Stable Hidden Quantum State in an Electronic Crystal. Science, 344(6180):177-180, 11, 2014. Cited on pages 1, 43, 50, 65, 77 and 97.

[8] V. R. Morrison, R. P. Chatelain, K. L. Tiwari, A. Hendaoui, A. Bruhács, M. Chaker, and B. J. Siwick. A photoinduced metal-like phase of monoclinic $\mathrm{VO}_{2}$ revealed by ultrafast electron diffraction. Science, 346(6208):445-448, 24, 2014. Cited on page 1. 
[9] E. Beaurepaire, J.-C. Merle, A. Daunois, and J.-Y. Bigot. Ultrafast Spin Dynamics in Ferromagnetic Nickel. Physical Review Letters, 76(22):4250-4253, 27, 1996. Cited on pages 1 and 2.

[10] H. E. Elsayed-Ali, T. B. Norris, M. A. Pessot, and G. A. Mourou. Time-resolved observation of electron-phonon relaxation in copper. Physical Review Letters, 58(12):1212-1215, 23, 1987. Cited on page 1.

[11] J. Demsar, K. Biljaković, and D. Mihailovic. Single Particle and Collective Excitations in the One-Dimensional Charge Density Wave Solid $\mathrm{K}_{0.3} \mathrm{MoO}_{3}$ Probed in Real Time by Femtosecond Spectroscopy. Physical Review Letters, 83(4):800-803, 26, 1999. Cited on pages 1 and 98 .

[12] T. Rohwer, S. Hellmann, M. Wiesenmayer, C. Sohrt, A. Stange, B. Slomski, A. Carr, Y. Liu, L. M. Avila, M. Kalläne, S. Mathias, L. Kipp, K. Rossnagel, and M. Bauer. Collapse of long-range charge order tracked by time-resolved photoemission at high momenta. Nature, 471(7339):490-493, 2011. Cited on page 1.

[13] R. Yusupov, T. Mertelj, V. V. Kabanov, S. Brazovskii, P. Kusar, J.-H. Chu, I. R. Fisher, and D. Mihailovic. Coherent dynamics of macroscopic electronic order through a symmetry breaking transition. Nature Physics, 6(9):681-684, 2010. Cited on page 1.

[14] S. Vogelgesang, G. Storeck, J. G. Horstmann, T. Diekmann, M. Sivis, S. Schramm, K. Rossnagel, S. Schäfer, and C. Ropers. Phase ordering of charge density waves traced by ultrafast low-energy electron diffraction. Nature Physics, 14(2):184-190, 6, 2017. Cited on pages $1,3,25,27,28,29,36,50,64,66,67,68,73,77,81,88$ and 97.

[15] D. Mihailovic. The Importance of Topological Defects in Photoexcited Phase Transitions Including Memory Applications:11, 2019. Cited on pages 1 and 98.

[16] A. Mann, E. Baldini, A. Odeh, A. Magrez, H. Berger, and F. Carbone. Probing the coupling between a doublon excitation and the charge-density wave in $\mathrm{TaS}_{2}$ by ultrafast optical spectroscopy. Physical Review B, 94(11):115122, 9, 2016. Cited on pages 2 and 66.

[17] C. Giannetti, M. Capone, D. Fausti, M. Fabrizio, F. Parmigiani, and D. Mihailovic. Ultrafast optical spectroscopy of strongly correlated materials and high-temperature superconductors: a non-equilibrium approach. Advances in Physics, 65(2):58-238, 3, 2016. Cited on page 2.

[18] M. Bauer, A. Marienfeld, and M. Aeschlimann. Hot electron lifetimes in metals probed by time-resolved two-photon photoemission. Progress in Surface Science, 90(3):319-376, 2015. Cited on page 2. 
[19] M. Wolf. Femtosecond dynamics of electronic excitations at metal surfaces. Surface Science, 377-379:343-349, 1997. Cited on page 2.

[20] S. L. Johnson, P. Beaud, E. Vorobeva, C. J. Milne, é. D. Murray, S. Fahy, and G. Ingold. Directly Observing Squeezed Phonon States with Femtosecond X-Ray Diffraction. Physical Review Letters, 102(17), 27, 2009. Cited on page 2.

[21] V. Juvé, M. Holtz, F. Zamponi, M. Woerner, T. Elsaesser, and A. Borgschulte. Field-Driven Dynamics of Correlated Electrons in $\mathrm{LiH}$ and NaBH 4 Revealed by Femtosecond X-Ray Diffraction. Physical Review Letters, 111(21):217401, 19, 2013. Cited on page 2.

[22] D. M. Fritz, D. A. Reis, B. Adams, R. A. Akre, J. Arthur, C. Blome, P. H. Bucksbaum, A. L. Cavalieri, S. Engemann, S. Fahy, R. W. Falcone, P. H. Fuoss, K. J. Gaffney, M. J. George, J. Hajdu, M. P. Hertlein, P. B. Hillyard, M. Horn-von Hoegen, M. Kammler, J. Kaspar, R. Kienberger, P. Krejcik, S. H. Lee, A. M. Lindenberg, B. McFarland, D. Meyer, T. Montagne, E. D. Murray, A. J. Nelson, M. Nicoul, R. Pahl, J. Rudati, H. Schlarb, D. P. Siddons, K. Sokolowski-Tinten, T. Tschentscher, D. von der Linde, and J. B. Hastings. Ultrafast Bond Softening in Bismuth: Mapping a Solid's Interatomic Potential with X-rays. Science, 315(5812):633-636, 2, 2007. Cited on page 2.

[23] P. Baum, D.-S. Yang, and A. H. Zewail. 4D Visualization of Transitional Structures in Phase Transformations by Electron Diffraction. Science, 318(5851):788-792, 2, 2007. Cited on pages 2 and 56 .

[24] M. Eichberger, H. Schäfer, M. Krumova, M. Beyer, J. Demsar, H. Berger, G. Moriena, G. Sciaini, and R. J. D. Miller. Snapshots of cooperative atomic motions in the optical suppression of charge density waves. Nature, 468(7325):799-802, 9, 2010. Cited on pages $2,50,51,52,64,65,66,67,68$ and 73.

[25] T. Aruga. Charge-density waves on metal surfaces. Journal of Physics: Condensed Matter, 14(35):8393-8414, 9, 2002. Cited on pages 2, 14 and 15.

[26] G. Grüner. Density waves in solids. Reading, Mass. Addison-Wesley Pub. Co., Advanced Book Program, 1994.

[27] U. Hofer. Time-Resolved Coherent Photoelectron Spectroscopy of Quantized Electronic States on Metal Surfaces. Science, 277(5331):1480-1482, 5, 1997. Cited on page 2.

[28] E. Knoesel, A. Hotzel, and M. Wolf. Temperature dependence of surface state lifetimes, dephasing rates and binding energies on $\mathrm{Cu}(111)$ studied with time-resolved photoemission. 
Journal of Electron Spectroscopy and Related Phenomena, 88-91:577-584, 1998. Cited on page 2.

[29] F. Bechstedt. Principles of Surface Physics. Berlin, Heidelberg. Springer Berlin Heidelberg, 2003.

[30] K. Oura, V. G. Lifshits, A. A. Saranin, A. V. Zotov, and M. Katayama. Surface Science: An Introduction. Springer Science \& Business Media, 2003.

[31] J. M. Kosterlitz. Ordering, metastability and phase transitions in two-dimensional systems. J. Phys. C, 6:1181-1203, 1973. Cited on page 2.

[32] R. E. Peierls. Quantum Theory of Solids. Clarendon Press, 1955.

[33] H. Fröhlich. On the theory of superconductivity: the one-dimensional case. Proceedings of the Royal Society of London. Series A. Mathematical and Physical Sciences, 223(1154):296305, 6, 1954. Cited on pages 2, 9, 18, 19 and 64 .

[34] S. Hellmann, T. Rohwer, M. Kalläne, K. Hanff, C. Sohrt, A. Stange, A. Carr, M. Murnane, H. Kapteyn, L. Kipp, M. Bauer, and K. Rossnagel. Time-domain classification of chargedensity-wave insulators. Nature Communications, 3(1):1069, 2012. Cited on pages 2, 50, $51,53,65,66,67,75$ and 76.

[35] W. A. Little. Possibility of Synthesizing an Organic Superconductor. Physical Review, 134(6):A1416-A1424, 15, 1964. Cited on page 2.

[36] W. Lee, Y. Chuang, R. Moore, Y. Zhu, L. Patthey, M. Trigo, D. Lu, P. Kirchmann, O. Krupin, M. Yi, M. Langner, N. Huse, J. Robinson, Y. Chen, S. Zhou, G. Coslovich, B. Huber, D. Reis, R. Kaindl, R. Schoenlein, D. Doering, P. Denes, W. Schlotter, J. Turner, S. Johnson, M. Först, T. Sasagawa, Y. Kung, A. Sorini, A. Kemper, B. Moritz, T. Devereaux, D.-H. Lee, Z. Shen, and Z. Hussain. Phase fluctuations and the absence of topological defects in a photo-excited charge-ordered nickelate. Nature Communications, 3(1), 2012. Cited on pages 2, 42, 68 and 77.

[37] H. Y. Liu, I. Gierz, J. C. Petersen, S. Kaiser, A. Simoncig, A. L. Cavalieri, C. Cacho, I. C. E. Turcu, E. Springate, F. Frassetto, L. Poletto, S. S. Dhesi, Z.-A. Xu, T. Cuk, R. Merlin, and A. Cavalleri. Possible observation of parametrically amplified coherent phasons in $\mathrm{K}_{0.3} \mathrm{MoO}_{3}$ using time-resolved extreme-ultraviolet angle-resolved photoemission spectroscopy. Physical Review B, 88(4), 2, 2013. Cited on pages 2, 64, 77 and 98. 
[38] A. Zong, A. Kogar, Y.-Q. Bie, T. Rohwer, C. Lee, E. Baldini, E. Ergeçen, M. B. Yilmaz, B. Freelon, E. J. Sie, H. Zhou, J. Straquadine, P. Walmsley, P. E. Dolgirev, A. V. Rozhkov, I. R. Fisher, P. Jarillo-Herrero, B. V. Fine, and N. Gedik. Evidence for topological defects in a photoinduced phase transition. Nature Physics, 15:27-31, 15, 2018. Cited on pages 2, $42,53,66,68,73,77$ and 98 .

[39] G.-H. Gweon, J. D. Denlinger, J. A. Clack, J. W. Allen, C. G. Olson, E. DiMasi, M. C. Aronson, B. Foran, and S. Lee. Direct Observation of Complete Fermi Surface, Imperfect Nesting, and Gap Anisotropy in the High-Temperature Incommensurate Charge-DensityWave Compound SmTe 3. Physical Review Letters, 81(4):886-889, 27, 1998. Cited on pages 2 and 64 .

[40] J. P. Pouget, B. Hennion, C. Escribe-Filippini, and M. Sato. Neutron-scattering investigations of the Kohn anomaly and of the phase and amplitude charge-density-wave excitations of the blue bronze $\mathrm{K}_{0.3} \mathrm{MoO}_{3}$. Physical Review B, 43(10):8421-8430, 1, 1991. Cited on pages 2 and 64 .

[41] F. Denoyer, F. Comès, A. F. Garito, and A. J. Heeger. X-Ray-Diffuse-Scattering Evidence for a Phase Transition in Tetrathiafulvalene Tetracyanoquinodimethane (TTF-TCNQ). Physical Review Letters, 35(7):445-449, 18, 1975. Cited on page 2.

[42] C. B. Scruby, P. M. Williams, and G. S. Parry. The role of charge density waves in structural transformations of 1T-TaS 2 . Philosophical Magazine, 31(2):255-274, 1975. Cited on pages $2,43,48,65$ and 84 .

[43] H. W. Yeom, S. Takeda, E. Rotenberg, I. Matsuda, K. Horikoshi, J. Schaefer, C. M. Lee, S. D. Kevan, T. Ohta, T. Nagao, and S. Hasegawa. Instability and Charge Density Wave of Metallic Quantum Chains on a Silicon Surface. Physical Review Letters, 82(24):4898-4901, 14, 1999. Cited on pages 3 and 97.

[44] T. Nakagawa, G. I. Boishin, H. Fujioka, H. W. Yeom, I. Matsuda, N. Takagi, M. Nishijima, and T. Aruga. Fermi Surface Nesting and Structural Transition on a Metal Surface: In / $\mathrm{Cu}(001)$. Physical Review Letters, 86(5):854-857, 29, 2001. Cited on pages 3 and 97.

[45] K. Swamy, A. Menzel, R. Beer, and E. Bertel. Charge-Density Waves in Self-Assembled Halogen-Bridged Metal Chains. Physical Review Letters, 86(7):1299-1302, 12, 2001. Cited on pages 3 and 97. 
[46] M. Gulde, S. Schweda, G. Storeck, M. Maiti, H. K. Yu, A. M. Wodtke, S. Schafer, and C. Ropers. Ultrafast low-energy electron diffraction in transmission resolves polymer/graphene superstructure dynamics. Science, 345(6193):200-204, 11, 2014. Cited on pages 3, 27, 28, 29, 56, 60, 64 and 66.

[47] G. Storeck, S. Vogelgesang, M. Sivis, S. Schäfer, and C. Ropers. Nanotip-based photoelectron microgun for ultrafast LEED. Structural Dynamics, 4(4):044024, 2017. Cited on pages $3,4,28,29,64,66$ and 78.

[48] J. G. Horstmann. Coherent control of a surface structural phase transition. Nature (accepted), 2020. Cited on pages 3, 64, 66 and 97.

[49] G. Storeck, J. G. Horstmann, T. Diekmann, S. Vogelgesang, G. von Witte, S. V. Yalunin, K. Rossnagel, and C. Ropers. Structural dynamics of incommensurate charge-density waves tracked by ultrafast low-energy electron diffraction. Structural Dynamics, 7(3):034304, 2020. Cited on pages 3, 4 and 88 .

[50] B. Wit, O. Bunjes, M. Wenderoth, and C. Ropers. Structure and Non-Equilibrium HeatTransfer of a Physisorbed Molecular Layer on Graphene (accepted). Advanced Materials Interfaces, 2020. Cited on pages 3 and 27.

[51] R. Peierls. More surprises in theoretical physics. Princeton University Press, 1991.

[52] R. Peierls. Bemerkungen über umwandlungstemperaturen. Helv. Phys. Acta, 7(2):81, 1934. Cited on page 5 .

[53] N. W. Ashcroft, N. D. Mermin, and D. Wei. Solid State Physics. Cengage Learning Asia Pte Limited, 2016.

[54] J. Solyom. Fundamentals of the Physics of Solids, Volume 3 - Normal, Broken- Symmetry, and Correlated Systems. Springer Science $\backslash \&$ Business Media, 2010.

[55] S. Kagoshima, H. Nagasawa, and T. Sambongi. One-Dimensional Conductors. Berlin, Heidelberg. Springer, 1988.

[56] S. Vogelgesang. Ultrafast low-energy electron diffraction at surfaces. PhD thesis. GeorgAugust-Universität Göttingen, 2019. Cited on pages 7, 22, 24, 28 and 47.

[57] B. Mihaila. Lindhard function of a d-dimensional Fermi gas. arXiv preprint arXiv:1111.5337, 2011. Cited on page 7.

[58] S. K. Chan and V. Heine. Spin density wave and soft phonon mode from nesting Fermi surfaces. Journal of Physics F: Metal Physics, 3(4):795-809, 1973. Cited on pages 7 and 15. 
[59] C. Herring. Magnetism: a treatise on modern theory and materials. 4. Exchange interactions among itinerant electrons. Academic Press, 1966.

[60] J. M. Ziman. Principles of the Theory of Solids. Cambridge University Press, 1972.

[61] G. Grimvall. The electron-phonon interaction in metals. North-Holland Pub. Co., 1981.

[62] F. Giustino. Electron-phonon interactions from first principles. Reviews of Modern Physics, 89(1):015003, 16, 2017. Cited on page 9.

[63] P. L. Taylor and O. Heinonen. A quantum approach to condensed matter physics. Cambridge, UK ; New York, NY. Cambridge University Press, 2002.

[64] A. Altland and B. D. Simons. Condensed Matter Field Theory. Cambridge University Press, 2010.

[65] E. Pavarini, E. Koch, R. Scalettar, and R. Martin. The Physics of Correlated Insulators, Metals, and Superconductors Lecture Notes of the Autumn School on Correlated Electrons 2017. Forschungszentrum Jülich GmbH, 2017.

[66] G. Czycholl. Theoretische Festkörperphysik: von den klassischen Modellen zu modernen Forschungsthemen. 3. Auflage edition, Berlin. Springer, 2008.

[67] P. Aynajian. Electron-phonon interaction in conventional and unconventional superconductors. Heidelberg ; New York. Springer, 2010.

[68] H.Z. Cummins. Experimental studies of structurally incommensurate crystal phases. Physics Reports, 185(5):211-409, 1990. Cited on pages 11, 13, 16, 19, 21 and 40.

[69] J. D. Axe. Incommensurate Structures. Philosophical Transactions of the Royal Society B: Biological Sciences, 290(1043):593-603, 20, 1980. Cited on pages 13 and 98.

[70] S. v. Smaalen. Incommensurate crystallography. Oxford ; New York. Oxford University Press, 2007.

[71] P. Monceau. Electronic crystals: an experimental overview. Advances in Physics, 61(4):325581, 2012. Cited on pages 13, 19, 20 and 96.

[72] K. Rossnagel. On the origin of charge-density waves in select layered transition-metal dichalcogenides. Journal of Physics: Condensed Matter, 23(21):213001, 1, 2011. Cited on pages $14,15,43,48$ and 65 .

[73] M. D. Johannes and I. I. Mazin. Fermi surface nesting and the origin of charge density waves in metals. Physical Review B, 77(16), 30, 2008. Cited on page 14. 
[74] J.-P. Pouget. The Peierls instability and charge density wave in one-dimensional electronic conductors. Comptes Rendus Physique, 17(3):332-356, 2016. Cited on pages 14 and 20.

[75] P. C. Snijders and H. H. Weitering. Colloquium : Electronic instabilities in self-assembled atom wires. Reviews of Modern Physics, 82(1):307-329, 12, 2010. Cited on page 14.

[76] C. Sohrt, A. Stange, M. Bauer, and K. Rossnagel. How fast can a Peierls-Mott insulator be melted? Faraday Discuss., 171:243-257, 7, 2014. Cited on pages 15, 43, 48, 49, 50, 64, 65, 66 and 76.

[77] W. L. McMillan. Landau theory of charge-density waves in transition-metal dichalcogenides. Physical Review B, 12(4):1187-1196, 15, 1975. Cited on pages 16, 23, 66 and 67.

[78] W. L. McMillan. Theory of discommensurations and the commensurate-incommensurate charge-density-wave phase transition. Physical Review B, 14(4):1496-1502, 15, 1976. Cited on pages $16,21,23,44$ and 66 .

[79] A. Beekman, L. Rademaker, and J. van Wezel. An introduction to spontaneous symmetry breaking. SciPost Physics Lecture Notes. Publisher: Stichting SciPost, 2019. Cited on page 16.

[80] P. M. Chaikin and T. C. Lubensky. Principles of condensed matter physics. Cambridge ; New York, NY, USA. Cambridge University Press, 1995.

[81] N. Goldenfeld. Lectures on Phase Transitions and the Renormalization Group. 1 edition, CRC Press, 1992.

[82] I. P. Ivanov. General two-order-parameter Ginzburg-Landau model with quadratic and quartic interactions. Physical Review E, 79(2):021116, 2018. Cited on page 16.

[83] F. Schwabl. Statistische Mechanik. Springer-Verlag, 2013.

[84] J. Ollivier, J. Etrillard, B. Toudic, C. Ecolivet, P. Bourges, and A. P. Levanyuk. Direct Observation of a Phason Gap in an Incommensurate Molecular Compound. Physical Review Letters, 81(17):3667-3670, 26, 1998. Cited on pages 17, 19, 20 and 21.

[85] S. Weinberg. The Quantum Theory of Fields. Cambridge University Press, 1995.

[86] T. W. B. Kibble. Topology of cosmic domains and strings. Journal of Physics A: Mathematical and General, 9(8):1387-1398, 1976. Cited on pages 17 and 64.

[87] W. H. Zurek. Cosmological experiments in superfluid helium? Nature, 317(6037):505-508, 1985. Cited on pages 17 and 64. 
[88] A. Auerbach. Interacting Electrons and Quantum Magnetism. Springer Science \& Business Media, 2012.

[89] P. W. Higgs. Broken Symmetries and the Masses of Gauge Bosons. Physical Review Letters, 13(16):508-509, 19, 1964. Cited on pages 17 and 64.

[90] Y. Nambu. Quasi-Particles and Gauge Invariance in the Theory of Superconductivity. Physical Review, 117(3):648-663, 1, 1960. Cited on pages 17 and 64.

[91] F. Strocchi. Symmetry breaking. 2nd ed edition, Berlin ; New York. Springer, 2008.

[92] P. Lee, T. Rice, and P. Anderson. Conductivity from charge or spin density waves. Solid State Communications, 14(8). Publisher: Elsevier:703-709, 1974. Cited on pages 17, 18 and 19.

[93] J. Axe. Incommensurate structural phase transformations. Proceedings of the conference on neutron scattering. Volume I (ed. R. M. Moon), 1976. Cited on page 18.

[94] A. W. Overhauser. Observability of Charge-Density Waves by Neutron Diffraction. Physical Review B, 3:3173, 1971. Cited on pages 18, 19, 39, 40, 66, 68 and 77.

[95] G. F. Giuliani and A. W. Overhauser. Structure factor of a charge-density wave. Physical Review B, 23(8):3737-3743, 15, 1981. Cited on pages 18, 40, 66, 68 and 77.

[96] C. Schlenker, ed. Low-Dimensional Electronic Properties of Molybdenum Bronzes and Oxides. Red. by F. Lévy and E. Mooser. Vol. 11. Physics and Chemistry of Materials with Low-Dimensional Structures. Dordrecht: Springer Netherlands, 1989. Cited on pages 18, 19 and 20.

[97] R. Currat. Neutron scattering studies of incommensurate systems. Ferroelectrics, 236(1):1122, 2000. Cited on page 19.

[98] L. Degiorgi, B. Alavi, G. Mihály, and G. Grüner. Complete excitation spectrum of chargedensity waves: Optical experiments on $\mathrm{K}_{0.3} \mathrm{MoO}_{3}$. Physical Review B, 44(15):7808-7819, 15, 1991. Cited on page 20.

[99] P. A. Lee and T. M. Rice. Electric field depinning of charge density waves. Physical Review $B, 19(8): 3970-3980,15,1979$. Cited on pages 20 and 96.

[100] A. A. Sinchenko, P. Lejay, and P. Monceau. Sliding charge-density wave in two-dimensional rare-earth tellurides. Physical Review B, 85(24):241104, 25, 2012. Cited on page 20. 
[101] H. Yao, J. A. Robertson, E.-A. Kim, and S. A. Kivelson. Theory of stripes in quasi-twodimensional rare-earth tellurides. Physical Review B, 74(24):245126, 28, 2006. Cited on page 20 .

[102] F. DiSalvo and R. Fleming. Search for a sliding charge density wave in layered compounds. Solid State Communications, 35(9):685-687, 1980. Cited on page 20.

[103] S. M. Shapiro, P. Vorderwisch, K. Habicht, K. Hradil, and H. Schneider. Observation of phasons in the magnetic shape memory alloy $\mathrm{Ni}_{2} \mathrm{MnGa}$. Europhysics Letters (EPL), 77(5):56004, 2007. Cited on pages 20 and 21.

[104] M. Quilichini and R. Currat. Neutron evidence for an overdamped phason branch in incommensurate K2SeO4. Solid State Communications, 48(12):1011-1015, 1983. Cited on page 20.

[105] R. Zeyher and W. Finger. Phason Dynamics of Incommensurate Crystals. Physical Review Letters, 49(25):1833-1837, 20, 1982. Cited on page 20.

[106] R. Blinc and A. Levanyuk. Incommensurate phases in dielectrics. North-Holland Amsterdam, 1986.

[107] J. Hlinka, M. Quilichini, R. Currat, and J. F. Legrand. Phason dispersion in the incommensurate phase of betaine calcium chloride dihydrate. Journal of Physics: Condensed Matter, 9(7):1461-1475, 17, 1997. Cited on page 20.

[108] P. Launois, F. Moussa, M. H. Lemée-Cailleau, and H. Cailleau. Phase and amplitude modes of the $n=4$ incommensurate phase II of biphenyl: Neutron scattering experiments and theoretical results. Physical Review B, 40(7):5042-5055, 1, 1989. Cited on page 20.

[109] R. Currat, L. Bernard, and P. Delamoye. Incommensurate phase in beta- $\mathrm{ThBr}_{4}$. Incommensurate phases in dielectrics. 1986. Cited on page 21.

[110] S. Ravy, H. Requardt, D. Le Bolloc'h, P. Foury-Leylekian, J.-P. Pouget, R. Currat, P. Monceau, and M. Krisch. Inelastic x-ray scattering study of charge-density-wave dynamics in the $\mathrm{Rb}_{0.3} \mathrm{MoO}_{3}$ blue bronze. Physical Review B, 69(11), 18, 2004. Cited on page 21.

[111] G. Travaglini, I. Mörke, and P. Wachter. CDW evidence in one-dimensional $\mathrm{K}_{0.3} \mathrm{MoO}_{3}$ by means of Raman scattering. Solid State Communications, 45(3). Publisher: Elsevier:289292, 1983. Cited on page 21.

[112] H. Requardt, J. E. Lorenzo, P. Monceau, R. Currat, and M. Krisch. Dynamics in the chargedensity-wave system $\mathrm{NbSe} 3$ using inelastic x-ray scattering with meV energy resolution. Physical Review B, 66(21), 12, 2002. Cited on page 21. 
[113] B. Hennion, J. P. Pouget, and M. Sato. Charge-density-wave phase elasticity of the blue bronze. Physical Review Letters, 68(15):2374-2377, 13, 1992. Cited on page 21.

[114] J. Odin, J. Lasjaunias, K. Biljaković, K. Hasselbach, and P. Monceau. Low temperature specific heat of blue bronze $\mathrm{K}_{0.3} \mathrm{MoO}_{3}$. The European Physical Journal B, 24(3):315-322, 2001. Cited on page 21.

[115] K. Nakanishi and H. Shiba. Domain-Like Incommensurate Charge-Density-Wave States and Collective Modes. Journal of the Physical Society of Japan, 1978. Cited on pages 21, 44, 45, 47 and 77.

[116] P. Bak. Commensurate phases, incommensurate phases and the devil's staircase. Reports on Progress in Physics, 45(6):587-629, 1, 1982. Cited on pages 22, 23, 24 and 98.

[117] Y. I. Frenkel and T. Kontorova. The model of dislocation in solid body. Zh. Eksp. Teor. Fiz, 8(1340), 1938. Cited on page 23.

[118] F. C. Frank and J. Van der Merwe. One-dimensional dislocations. II. Misfitting monolayers and oriented overgrowth. Proceedings of the Royal Society of London. Series A. Mathematical and Physical Sciences, 198(1053). Publisher: The Royal Society London:216-225, 1949. Cited on page 23.

[119] G. Theodorou and T. Rice. Statics and dynamics of incommensurate lattices. Physical Review B, 18(6). Publisher: APS:2840, 1978. Cited on page 23.

[120] S. Aubry. Chapter 8 of Solitons and Condensed Matter Physics editors: Bishop AR and Schneider T. Springer-Verlag, Berlin, 1979.

[121] J. M. Greene. A method for determining a stochastic transition. Journal of Mathematical Physics, 20(6). Publisher: American Institute of Physics:1183-1201, 1979. Cited on page 23.

[122] P. Bak. Chaotic behavior and incommensurate phases in the anisotropic Ising model with competing interactions. Physical Review Letters, 46(13). Publisher: APS:791, 1981. Cited on page 23 .

[123] M. Henzler and W. Göpel. Oberflächenphysik des Festkörpers. Wiesbaden. Vieweg+Teubner Verlag, 1994.

[124] T. Fauster and M. A. Schneider. Oberflächenphysik: Grundlagen und Methoden. Oldenbourg Verlag, 2013. 
[125] M. A. Van Hove, W. H. Weinberg, and C.-M. Chan. Low-energy electron diffraction: experiment, theory and surface structure determination. Softcover reprint of the hardcover 1. edition 1986 edition, Berlin. Springer, 1986.

[126] M. P. Seah and W. A. Dench. Quantitative electron spectroscopy of surfaces: A standard data base for electron inelastic mean free paths in solids. Surface and Interface Analysis, 1(1):2-11, 1979. Cited on page 26.

[127] L. J. Clarke. Surface crystallography: an introduction to low energy electron diffraction. Wiley, 1985.

[128] R. Bormann, S. Strauch, S. Schäfer, and C. Ropers. An ultrafast electron microscope gun driven by two-photon photoemission from a nanotip cathode. Journal of Applied Physics, 118(17):173105, 6, 2015. Cited on pages 29, 56, 58, 60 and 89.

[129] G. von Witte, T. Kißlinger, J. G. Horstmann, K. Rossnagel, M. A. Schneider, C. Ropers, and L. Hammer. Surface structure and stacking of the commensurate (13x13)R13.9 charge density wave phase of 1T-TaS2 ( 0001 ). Physical Review B, 100(15):155407, 8, 2019. Cited on pages $29,46,69$ and 84 .

[130] S. Hunklinger. Festkörperphysik. 2., verb. Aufl edition, München. Oldenbourg, 2009.

[131] A. Ichimiya and P. I. Cohen. Reflection high-energy electron diffraction. Cambridge, U.K. ; New York. Cambridge University Press, 2004.

[132] G. A. Somorjai and Y. Li. Introduction to surface chemistry and catalysis. John Wiley \& Sons, 2010.

[133] H. Busch and M. Henzler. Quantitative evaluation of terrace width distributions from LEED measurements. Surface science, 167(2). Publisher: Elsevier:534-548, 1986. Cited on page 36.

[134] P. Debye. Zur theorie der spezifischen wärmen. Annalen der Physik, 344(14). Publisher: Wiley Online Library:789-839, 1912. Cited on page 37.

[135] I. Waller. Zur Theorie der Röntgenreflexion. Annalen der Physik, 384(3). Publisher: Wiley Online Library:261-272, 1926. Cited on page 37.

[136] M. Holt, P. Zschack, H. Hong, M. Y. Chou, and T.-C. Chiang. X-Ray Studies of Phonon Softening in TiSe 2. Physical Review Letters, 86(17):3799-3802, 23, 2001. Cited on page 37. 
[137] M. Trigo, J. Chen, V. H. Vishwanath, Y. M. Sheu, T. Graber, R. Henning, and D. A. Reis. Imaging nonequilibrium atomic vibrations with x-ray diffuse scattering. Physical Review B, 82(23):235205, 10, 2010. Cited on page 37.

[138] M. J. Stern, L. P. René de Cotret, M. R. Otto, R. P. Chatelain, J.-P. Boisvert, M. Sutton, and B. J. Siwick. Mapping momentum-dependent electron-phonon coupling and nonequilibrium phonon dynamics with ultrafast electron diffuse scattering. Physical Review B, 97(16):165416, 12, 2018. Cited on pages 37, 38 and 68.

[139] T. P. Devereaux, A. M. Shvaika, K. Wu, K. Wohlfeld, C. J. Jia, Y. Wang, B. Moritz, L. Chaix, W.-S. Lee, Z.-X. Shen, G. Ghiringhelli, and L. Braicovich. Directly Characterizing the Relative Strength and Momentum Dependence of Electron-Phonon Coupling Using Resonant Inelastic X-Ray Scattering. Physical Review X, 6(4):041019, 25, 2016. Cited on page 38 .

[140] H. Yan, D. Song, K. F. Mak, I. Chatzakis, J. Maultzsch, and T. F. Heinz. Time-resolved Raman spectroscopy of optical phonons in graphite: Phonon anharmonic coupling and anomalous stiffening. Physical Review B, 80(12):121403, 17, 2009. Cited on page 38.

[141] L. Waldecker, R. Bertoni, H. Hübener, T. Brumme, T. Vasileiadis, D. Zahn, A. Rubio, and R. Ernstorfer. Momentum-Resolved View of Electron-Phonon Coupling in Multilayer WSe 2. Physical Review Letters, 119(3), 21, 2017. Cited on pages 38 and 68.

[142] M. Harb, H. Enquist, A. Jurgilaitis, F. T. Tuyakova, A. N. Obraztsov, and J. Larsson. Phonon-phonon interactions in photoexcited graphite studied by ultrafast electron diffraction. Physical Review B, 93(10):104104, 7, 2016. Cited on pages 38 and 76.

[143] T. Chase, M. Trigo, A. H. Reid, R. Li, T. Vecchione, X. Shen, S. Weathersby, R. Coffee, N. Hartmann, D. A. Reis, X. J. Wang, and H. A. Dürr. Ultrafast electron diffraction from non-equilibrium phonons in femtosecond laser heated Au films. Applied Physics Letters, 108(4):041909, 25, 2016. Cited on page 38.

[144] H. Kayser and H. M. Konen. Handbuch der spectroscopie. S. Hirzel, 1900.

[145] U. Dehlinger. XXXVII. Über die Verbreiterung der Debyelinien bei kaltbearbeiteten Metallen. Zeitschrift für Kristallographie-Crystalline Materials, 65(1). Publisher: De Gruyter Oldenbourg:615-631, 1927. Cited on page 38.

[146] R. Currat and T. Janssen. Excitations in incommensurate crystal phases. Solid State Physics, 1988. Cited on pages 39 and 42. 
[147] W. Minor, L. D. Chapman, S. N. Ehrlich, and R. Colella. Phason velocities in $\mathrm{TaS}_{2}$ by X-ray diffuse scattering. Physical Review B, 39(2):1360-1362, 15, 1989. Cited on pages 40, 42, 64, 73 and 77.

[148] M. A. Krivoglaz. Diffuse Scattering of X-rays and Neutrons by Fluctuations. Springer Berlin, 1996.

[149] J. D. Axe. Debye-Waller factors for incommensurate structures. Physical Review B, 21(10):41814190, 15, 1980. Cited on pages 40, 41, 42 and 77.

[150] A. Bruce, R. A. Cowley, B. Dorner, A. Muller, H. Thomas, and T. i. C. Physics; 23. Structural phase transitions. Taylor \& Francis London, 1981.

[151] Y. R. Wang and A. W. Overhauser. Thermal-diffuse scattering of $\mathrm{x}$ rays by phasons. Physical Review B, 39(2):1357-1359, 15, 1989. Cited on pages 42, 68, 95 and 96.

[152] W. Adlhart. Dynamic structure factors for excitations in modulated structures. Acta Crystallographica Section A, 38(4):498-504, 1, 1982. Cited on pages 42 and 77.

[153] T. A. Aslanyan, T. Shigenari, and K. Abe. Comment on the Debye-Waller factors for incommensurate structures. arXiv:cond-mat/0502638, 26, 2005. Cited on pages 42 and 77.

[154] L. D. Chapman and R. Colella. Experimental Evidence from X-Ray Diffraction for Phase Excitations in Solids. Physical Review Letters, 52(8):652-655, 20, 1984. Cited on pages 42, 48, 64 and 77.

[155] K.-H. Ehses. Phase Fluctuations in the IC-Phase of $\mathrm{Rb}_{2} \mathrm{ZnCl}_{4}$. Japanese Journal of Applied Physics, 24:793, S2 1, 1985. Cited on page 42.

[156] I. Aramburu, G. Madariaga, D. Grebille, J. Perez-Mato, and T. Breczewski. High-order diffraction satellites and temperature variation of the modulation in the incommensurate phase of $\mathrm{Rb}_{2} \mathrm{ZnCl}_{4}$. Journal de Physique I, 7(2). Publisher: EDP Sciences:371-383, 1997. Cited on page 42 .

[157] B. Sipos, A. F. Kusmartseva, A. Akrap, H. Berger, L. Forró, and E. Tutǐ̌s. From Mott state to superconductivity in $1 \mathrm{~T}-\mathrm{TaS}_{2}$. Nature Materials, 7(12):960-965, 2008. Cited on pages 43, 46 and 65.

[158] T. Ritschel. Druckabhängigkeit der Ladungsdichtewelle in $1 T-\mathrm{TaS}_{2} . \mathrm{PhD}$ thesis. Technische Universität Dresden, 2010. Cited on page 43.

[159] J. Wilson, F. Di Salvo, and S. Mahajan. Charge-density waves and superlattices in the metallic layered transition metal dichalcogenides. Advances in Physics, 24(2):117-201, 1975. Cited on pages $43,45,48$ and 61 . 
[160] H. Lin, W. Huang, K. Zhao, S. Qiao, Z. Liu, J. Wu, X. Chen, and S.-H. Ji. Scanning tunneling spectroscopic study of monolayer $1 \mathrm{~T}-\mathrm{TaS}_{2}$ and 1T-TaSe 2 . Nano Research, 13(1):133-137, 2020. Cited on pages 43 and 97.

[161] A. Spijkerman, J. L. de Boer, A. Meetsma, G. A. Wiegers, and S. van Smaalen. X-ray crystalstructure refinement of the nearly commensurate phase of $1 \mathrm{~T}-\mathrm{TaS}_{2}$ in $(3+2)$-dimensional superspace. Physical Review B, 56(21):13757-13767, 1, 1997. Cited on pages 43, 44, 45, 46, 47, 48, 65, 66 and 69.

[162] M. Kratochvilova, A. D. Hillier, A. R. Wildes, L. Wang, S.-W. Cheong, and J.-G. Park. The low-temperature highly correlated quantum phase in the charge-density-wave $1 \mathrm{~T}-\mathrm{TaS}_{2}$ compound. npj Quantum Materials, 2(1):42, 2017. Cited on page 44.

[163] S. Hellmann, M. Beye, C. Sohrt, T. Rohwer, F. Sorgenfrei, H. Redlin, M. Kalläne, M. Marczynski-Bühlow, F. Hennies, M. Bauer, A. Föhlisch, L. Kipp, W. Wurth, and K. Rossnagel. Ultrafast Melting of a Charge-Density Wave in the Mott Insulator 1T-TaS 2 . Physical Review Letters, 105(18), 27, 2010. Cited on pages 44, 50, 51, 64 and 66.

[164] D. E. Moncton, J. D. Axe, and F. J. DiSalvo. Neutron scattering study of the charge-density wave transitions in 2H-TaSe 2 and 2H-NbSe 2 . Physical Review B, 16(2):801-819, 15, 1977. Cited on page 44.

[165] K. Nakanishi and H. Shiba. Domain-like Incommensurate Charge-Density-Wave States and the First-Order Incommensurate-Commensurate Transitions in Layered Tantalum Dichalcogenides. II. 2H-Polytype. Journal of the Physical Society of Japan, 1978. Cited on pages 44, 45 and 47.

[166] K. Nakanishi and H. Shiba. Domain-like Incommensurate Charge-Density-Wave States and the First-Order Incommensurate-Commensurate Transitions in Layered Tantalum Dichalcogenides. I. 1T-Polytype. Journal of the Physical Society of Japan, 43(6):1839-1847, 1977. Cited on pages 44, 45, 47 and 66.

[167] K. Nakanishi, H. Takatera, Y. Yamada, and H. Shiba. The Nearly Commensurate Phase and Effect of Harmonics on the Successive Phase Transition in 1T-TaS 2 . Journal of the Physical Society of Japan, 43(5):1509-1517, 1977. Cited on pages 44, 45, 66 and 67.

[168] A. Zong, X. Shen, A. Kogar, L. Ye, C. Marks, D. Chowdhury, T. Rohwer, B. Freelon, S. Weathersby, R. Li, J. Yang, J. Checkelsky, X. Wang, and N. Gedik. Ultrafast manipulation of mirror domain walls in a charge density wave. Science Advances, 4(10):eaau5501, 2018. Cited on page 45. 
[169] R. Brouwer and F. Jellinek. The low-temperature superstructures of $1 \mathrm{~T}-\mathrm{TaSe}_{2}$ and $2 \mathrm{H}-\mathrm{TaSe}_{2}$. Physica B+C, 99(1):51-55, 1980. Cited on page 46.

[170] R. E. Thomson, B. Burk, A. Zettl, and J. Clarke. Scanning tunneling microscopy of the charge-density-wave structure in 1T-TaS 2 . Physical Review B, 49(24):16899, 1994. Cited on pages 46,47 and 48 .

[171] P. M. Williams, G. S. Parry, and C. B. Scrub. Diffraction evidence for the Kohn anomaly in 1T-TaS 2 . Philosophical Magazine, 29(3):695-699, 1974. Cited on page 48.

[172] F. Clerc, C. Battaglia, M. Bovet, L. Despont, C. Monney, H. Cercellier, M. G. Garnier, P. Aebi, H. Berger, and L. Forró. Lattice-distortion-enhanced electron-phonon coupling and Fermi surface nesting in 1T-TaS 2 . Physical Review B, 74(15), 17, 2006. Cited on page 48.

[173] J. Demsar, L. Forró, H. Berger, and D. Mihailovic. Femtosecond snapshots of gap-forming charge-density-wave correlations in quasi-two-dimensional dichalcogenides $1 \mathrm{~T}-\mathrm{TaS}_{2}$ and 2H-TaSe 2 . Physical Review B, 66(4), 28, 2002. Cited on pages 48, 50, 51, 64, 65, 66, 68 and 75.

[174] A. Thompson, R. Gamble, and J. Revelli. Transitions between semiconducting and metallic phases in 1-T TaS 2 . Solid State Communications, 9(13):981-985, 1971. Cited on page 48.

[175] F. J. Di Salvo, J. A. Wilson, B. G. Bagley, and J. V. Waszczak. Effects of doping on chargedensity waves in layer compounds. Physical Review B, 12(6):2220-2235, 15, 1975. Cited on page 48 .

[176] T. Ritschel, J. Trinckauf, K. Koepernik, B. Büchner, M. v. Zimmermann, H. Berger, Y. I. Joe, P. Abbamonte, and J. Geck. Orbital textures and charge density waves in transition metal dichalcogenides. Nature Physics, 11(4):328-331, 2015. Cited on pages 49, 65 and 96.

[177] T. Ritschel, H. Berger, and J. Geck. Stacking-driven gap formation in layered $1 T-\mathrm{TaS}_{2}$. Physical Review B, 98(19):195134, 26, 2018. Cited on pages 49 and 96.

[178] S. Uchida and S. Sugai. Infrared and Raman studies on commensurate CDW states in transition metal dichalcogenides. Physica B+C, 105(1):393-399, 1981. Cited on pages 49 and 64.

[179] A. S. Barker, J. A. Ditzenberger, and F. J. DiSalvo. Infrared study of the electronic instabilities in tantalum disulfide and tantalum diselenide. Physical Review B, 12(6):2049-2054, 15, 1975. Cited on page 49 . 
[180] D. R. Karecki and B. P. Clayman. Far-infrared reflection spectra of 1T-TaS 2 and 1T$\mathrm{TaSe}_{2}$ in commensurate and incommensurate charge-density-wave states. Physical Review B, 19(12):6367-6371, 15, 1979. Cited on page 49.

[181] J. Smith, J. Tsang, and M. Shafer. Raman spectra of several layer compounds with charge density waves. Solid State Communications, 19(4):283-286, 1976. Cited on page 49.

[182] J. Duffey, R. Kirby, and R. Coleman. Raman scattering from 1T-TaS 2 . Solid State Communications, 20(6):617-621, 1976. Cited on pages 49 and 64.

[183] K. R. A. Ziebeck, B. Dorner, W. G. Stirling, and R. Schöllhorn. Kohn anomaly in the $1 \mathrm{~T}_{2}$ phase of $\mathrm{TaS}_{2}, 1976$. Cited on pages 49 and 50.

[184] N. S. Luo, P. Ruggerone, J. P. Toennies, and G. Benedek. Electron-phonon coupling at metal surfaces probed by helium atom scattering. Physica Scripta, T49B:584-592, 1, 1993. Cited on page 49.

[185] Y. Machida, T. Hanashima, K. Ohkubo, K. Yamawaki, M. Tanaka, and S. Sasaki. Observation of Soft Phonon Modes in $1 T-\mathrm{TaS}_{2}$ by means of X-ray Thermal Diffuse Scattering. Journal of the Physical Society of Japan, 73(11):3064-3069, 15, 2004. Cited on page 49.

[186] I. Avigo, P. Zhou, M. Kalläne, K. Rossnagel, U. Bovensiepen, and M. Ligges. Excitation and Relaxation Dynamics of the Photo-Perturbed Correlated Electron System 1T-TaS 2 . Applied Sciences, 9(1):44, 2019. Cited on pages 50 and 66.

[187] L. Perfetti, P. A. Loukakos, M. Lisowski, U. Bovensiepen, H. Berger, S. Biermann, P. S. Cornaglia, A. Georges, and M. Wolf. Time Evolution of the Electronic Structure of 1T-TaS 2 through the Insulator-Metal Transition. Physical Review Letters, 97(6), 8, 2006. Cited on pages $50,64,65,66,67$ and 76.

[188] L. Perfetti, P. A. Loukakos, M. Lisowski, U. Bovensiepen, M. Wolf, H. Berger, S. Biermann, and A. Georges. Femtosecond dynamics of electronic states in the Mott insulator 1T-TaS 2 by time resolved photoelectron spectroscopy. New Journal of Physics, 10(5):053019, 14, 2008. Cited on pages 50, 64, 65 and 66.

[189] G. Lantz, C. Laulhé, S. Ravy, M. Kubli, M. Savoini, K. Tasca, E. Abreu, V. Esposito, M. Porer, A. Ciavardini, L. Cario, J. Rittmann, P. Beaud, and S. L. Johnson. Domain-size effects on the dynamics of a charge density wave in $1 \mathrm{~T}-\mathrm{TaS}_{2}$. Physical Review B, 96(22), 6, 2017. Cited on pages 50, 64, 67 and 77. 
[190] C. Laulhé, T. Huber, G. Lantz, A. Ferrer, S. O. Mariager, S. Grübel, J. Rittmann, J. A. Johnson, V. Esposito, A. Lübcke, L. Huber, M. Kubli, M. Savoini, V. L. R. Jacques, L. Cario, B. Corraze, E. Janod, G. Ingold, P. Beaud, S. L. Johnson, and S. Ravy. Ultrafast Formation of a Charge Density Wave State in $1 \mathrm{~T}-\mathrm{TaS}_{2}$ : Observation at Nanometer Scales Using Time-Resolved X-Ray Diffraction. Physical Review Letters, 118(24), 16, 2017. Cited on pages 50, 66 and 77 .

[191] K. Haupt, M. Eichberger, N. Erasmus, A. Rohwer, J. Demsar, K. Rossnagel, and H. Schwoerer. Ultrafast Metamorphosis of a Complex Charge-Density Wave. Phys. Rev. Lett.:5, 2016. Cited on pages 50, 53, 64, 66 and 67.

[192] T.-R. T. Han, F. Zhou, C. D. Malliakas, P. M. Duxbury, S. D. Mahanti, M. G. Kanatzidis, and C.-Y. Ruan. Exploration of metastability and hidden phases in correlated electron crystals visualized by femtosecond optical doping and electron crystallography. Science Advances, 1(5):e1400173-e1400173, 26, 2015. Cited on page 53.

[193] A. Kogar, A. Zong, P. E. Dolgirev, X. Shen, J. Straquadine, Y.-Q. Bie, X. Wang, T. Rohwer, I.-C. Tung, Y. Yang, et al. Light-induced charge density wave in LaTe 3. Nature Physics, 16(2). Publisher: Nature Publishing Group:159-163, 2020. Cited on pages 53, 77 and 98.

[194] N. Erasmus, M. Eichberger, K. Haupt, I. Boshoff, G. Kassier, R. Birmurske, H. Berger, J. Demsar, and H. Schwoerer. Ultrafast Dynamics of Charge Density Waves in $4 \mathrm{Hb}-\mathrm{TaSe}_{2}$ Probed by Femtosecond Electron Diffraction. Physical Review Letters, 109(16):167402, 18, 2012. Cited on pages 53, 64, 68 and 73.

[195] T.-R. T. Han, Z. Tao, S. D. Mahanti, K. Chang, C.-Y. Ruan, C. D. Malliakas, and M. G. Kanatzidis. Structural dynamics of two-dimensional charge-density waves in CeTe 3 investigated by ultrafast electron crystallography. Physical Review B, 86(7), 27, 2012. Cited on pages 53 and 64 .

[196] R. J. D. Miller. Femtosecond Crystallography with Ultrabright Electrons and X-rays: Capturing Chemistry in Action. Science, 343(6175):1108-1116, 7, 2014. Cited on page 55.

[197] A. Hanisch-Blicharski, A. Janzen, B. Krenzer, S. Wall, F. Klasing, A. Kalus, T. Frigge, M. Kammler, and M. Horn-von Hoegen. Ultra-fast electron diffraction at surfaces: From nanoscale heat transport to driven phase transitions. Ultramicroscopy, 127:2-8, 2013. Cited on page 55. 
[198] S. P. Weathersby, G. Brown, M. Centurion, T. F. Chase, R. Coffee, J. Corbett, J. P. Eichner, J. C. Frisch, A. R. Fry, M. Gühr, N. Hartmann, C. Hast, R. Hettel, R. K. Jobe, E. N. Jongewaard, J. R. Lewandowski, R. K. Li, A. M. Lindenberg, I. Makasyuk, J. E. May, D. McCormick, M. N. Nguyen, A. H. Reid, X. Shen, K. Sokolowski-Tinten, T. Vecchione, S. L. Vetter, J. Wu, J. Yang, H. A. Dürr, and X. J. Wang. Mega-electron-volt ultrafast electron diffraction at SLAC National Accelerator Laboratory. Review of Scientific Instruments, 86(7):073702, 2015. Cited on page 55.

[199] D. J. Flannigan and A. H. Zewail. 4D Electron Microscopy: Principles and Applications. Accounts of Chemical Research, 45(10):1828-1839, 16, 2012. Cited on page 55.

[200] J. S. Kim, T. LaGrange, B. W. Reed, M. L. Taheri, M. R. Armstrong, W. E. King, N. D. Browning, and G. H. Campbell. Imaging of Transient Structures Using Nanosecond in Situ TEM. Science, 321(5895):1472-1475, 12, 2008. Cited on page 55.

[201] K. Bücker, M. Picher, O. Crégut, T. LaGrange, B. Reed, S. Park, D. Masiel, and F. Banhart. Electron beam dynamics in an ultrafast transmission electron microscope with Wehnelt electrode. Ultramicroscopy, 171:8-18, 2016. Cited on page 55.

[202] L. Piazza, D. Masiel, T. LaGrange, B. Reed, B. Barwick, and F. Carbone. Design and implementation of a fs-resolved transmission electron microscope based on thermionic gun technology. Chemical Physics, 423:79-84, 2013. Cited on page 55.

[203] A. Feist, K. E. Echternkamp, J. Schauss, S. V. Yalunin, S. Schäfer, and C. Ropers. Quantum coherent optical phase modulation in an ultrafast transmission electron microscope. Nature, 521(7551):200-203, 14, 2015. Cited on pages 55 and 58.

[204] A. Feist, N. Bach, N. Rubiano da Silva, T. Danz, M. Möller, K. E. Priebe, T. Domröse, J. G. Gatzmann, S. Rost, J. Schauss, S. Strauch, R. Bormann, M. Sivis, S. Schäfer, and C. Ropers. Ultrafast transmission electron microscopy using a laser-driven field emitter: Femtosecond resolution with a high coherence electron beam. Ultramicroscopy, 176:63-73, 1, 2017. Cited on pages 55 and 60 .

[205] F. Carbone, O.-H. Kwon, and A. H. Zewail. Dynamics of Chemical Bonding Mapped by Energy-Resolved 4D Electron Microscopy. Science, 325(5937):181-184, 10, 2009. Cited on page 55.

[206] R. M. van der Veen, T. J. Penfold, and A. H. Zewail. Ultrafast core-loss spectroscopy in four-dimensional electron microscopy. Structural Dynamics, 2(2):024302, 2015. Cited on page 55. 
[207] H. E. Elsayed-Ali and J. W. Herman. Picosecond time-resolved surface-lattice temperature probe. Applied Physics Letters, 57(15):1508-1510, 8, 1990. Cited on page 55.

[208] M. Aeschlimann, E. Hull, J. Cao, C. A. Schmuttenmaer, L. G. Jahn, Y. Gao, H. E. ElsayedAli, D. A. Mantell, and M. R. Scheinfein. A picosecond electron gun for surface analysis. Review of Scientific Instruments, 66(2):1000-1009, 1995. Cited on page 55.

[209] S. Wall, B. Krenzer, S. Wippermann, S. Sanna, F. Klasing, A. Hanisch-Blicharski, M. Kammler, W. G. Schmidt, and M. Horn-von Hoegen. Atomistic Picture of Charge Density Wave Formation at Surfaces. Physical Review Letters, 109(18), 2, 2012. Cited on page 56.

[210] R. Karrer, H. J. Neff, M. Hengsberger, T. Greber, and J. Osterwalder. Design of a miniature picosecond low-energy electron gun for time-resolved scattering experiments. Review of Scientific Instruments, 72(12):4404-4407, 2001. Cited on page 56.

[211] C. Cirelli, M. Hengsberger, A. Dolocan, H. Over, J. Osterwalder, and T. Greber. Direct observation of space charge dynamics by picosecond low-energy electron scattering. $E P L$ (Europhysics Letters), 85(1):17010, 2009. Cited on page 56.

[212] A. Paarmann, M. Gulde, M. Müller, S. Schäfer, S. Schweda, M. Maiti, C. Xu, T. Hohage, F. Schenk, C. Ropers, and R. Ernstorfer. Coherent femtosecond low-energy single-electron pulses for time-resolved diffraction and imaging: A numerical study. Journal of Applied Physics, 112(11):113109, 2012. Cited on pages 56 and 58.

[213] P. Hommelhoff, Y. Sortais, A. Aghajani-Talesh, and M. A. Kasevich. Field Emission Tip as a Nanometer Source of Free Electron Femtosecond Pulses. Physical Review Letters, 96(7), 21, 2006. Cited on page 56.

[214] C. Ropers, D. R. Solli, C. P. Schulz, C. Lienau, and T. Elsaesser. Localized Multiphoton Emission of Femtosecond Electron Pulses from Metal Nanotips. Physical Review Letters, 98(4), 25, 2007. Cited on page 56.

[215] H. Yanagisawa, M. Hengsberger, D. Leuenberger, M. Klöckner, C. Hafner, T. Greber, and J. Osterwalder. Energy Distribution Curves of Ultrafast Laser-Induced Field Emission and Their Implications for Electron Dynamics. Physical Review Letters, 107(8):087601, 16, 2011. Cited on page 56.

[216] S. Lüneburg, M. Müller, A. Paarmann, and R. Ernstorfer. Microelectrode for energy and current control of nanotip field electron emitters. Applied Physics Letters, 103(21):213506, 18, 2013. Cited on pages 56 and 91. 
[217] B. Schröder, M. Sivis, R. Bormann, S. Schäfer, and C. Ropers. An ultrafast nanotip electron gun triggered by grating-coupled surface plasmons. Applied Physics Letters, 107(23):231105, 7, 2015. Cited on page 56.

[218] A. Căsăndruc, R. Bücker, G. Kassier, and R. J. D. Miller. Optical fiber-based photocathode. Applied Physics Letters, 109(9):091105, 29, 2016. Cited on pages 56 and 60.

[219] M. Müller, A. Paarmann, and R. Ernstorfer. Femtosecond electrons probing currents and atomic structure in nanomaterials. Nature Communications, 5:5292, 31, 2014. Cited on page 56.

[220] E. Quinonez, J. Handali, and B. Barwick. Femtosecond photoelectron point projection microscope. Review of Scientific Instruments, 84(10):103710, 2013. Cited on page 56.

[221] J. Vogelsang, J. Robin, B. J. Nagy, P. Dombi, D. Rosenkranz, M. Schiek, P. Groß, and C. Lienau. Ultrafast Electron Emission from a Sharp Metal Nanotaper Driven by Adiabatic Nanofocusing of Surface Plasmons. Nano Letters, 15(7):4685-4691, 8, 2015. Cited on page 56.

[222] G. F. Mancini, B. Mansart, S. Pagano, B. van der Geer, M. de Loos, and F. Carbone. Design and implementation of a flexible beamline for fs electron diffraction experiments. Nuclear Instruments and Methods in Physics Research Section A: Accelerators, Spectrometers, Detectors and Associated Equipment, 691:113-122, 2012. Cited on page 60.

[223] H. Park and J. M. Zuo. Direct measurement of transient electric fields induced by ultrafast pulsed laser irradiation of silicon. Applied Physics Letters, 94(25):251103, 22, 2009. Cited on page 60.

[224] J. Goldstone. Field theories with Superconductor solutions. Il Nuovo Cimento, 19(1):154164, 1961. Cited on page 64.

[225] J. Bardeen, L. N. Cooper, and J. R. Schrieffer. Theory of Superconductivity. Physical Review, 108(5):1175-1204, 1, 1957. Cited on page 64.

[226] T. Hirata and F. Ohuchi. Temperature dependence of the Raman spectra of 1T-TaS 2 . Solid State Communications, 117(6):361-364, 2001. Cited on page 64.

[227] J. C. Petersen, S. Kaiser, N. Dean, A. Simoncig, H. Y. Liu, A. L. Cavalieri, C. Cacho, I. C. E. Turcu, E. Springate, F. Frassetto, L. Poletto, S. S. Dhesi, H. Berger, and A. Cavalleri. Clocking the Melting Transition of Charge and Lattice Order in $1 \mathrm{~T}-\mathrm{TaS}_{2}$ with Ultrafast Extreme-Ultraviolet Angle-Resolved Photoemission Spectroscopy. Physical Review Letters, 107(17):177402, 18, 2011. Cited on pages 64, 65, 66 and 76. 
[228] C. Laulhé, L. Cario, B. Corraze, E. Janod, T. Huber, G. Lantz, S. Boulfaat, A. Ferrer, S. Mariager, J. Johnson, S. Grübel, A. Lübcke, G. Ingold, P. Beaud, S. Johnson, and S. Ravy. X-ray study of femtosecond structural dynamics in the 2D charge density wave compound 1T-TaS 2 . Physica B: Condensed Matter, 460:100-104, 2015. Cited on pages 64 and 67.

[229] C. W. Nicholson, A. Lücke, W. G. Schmidt, M. Puppin, L. Rettig, R. Ernstorfer, and M. Wolf. Beyond the molecular movie: Dynamics of bands and bonds during a photoinduced phase transition. Science, 362(6416):821-825, 16, 2018. Cited on page 64.

[230] X. Shi, W. You, Y. Zhang, Z. Tao, P. M. Oppeneer, X. Wu, R. Thomale, K. Rossnagel, M. Bauer, H. Kapteyn, and M. Murnane. Ultrafast electron calorimetry uncovers a new longlived metastable state in $1 T$ - $\mathrm{TaSe}_{2}$ mediated by mode-selective electron-phonon coupling. Science Advances, 5(3):eaav4449, 2019. Cited on pages 64, 65, 76 and 77.

[231] T.-R. T. Han. Ultrafast electron crystallography studies of charge-density waves materials and nanoscale ice. Michigan State University, 2015.

[232] I. Vaskivskyi, J. Gospodaric, S. Brazovskii, D. Svetin, P. Sutar, E. Goreshnik, I. A. Mihailovic, T. Mertelj, and D. Mihailovic. Controlling the metal-to-insulator relaxation of the metastable hidden quantum state in $1 \mathrm{~T}-\mathrm{TaS}_{2}$. Science Advances, 1(6):e1500168, 2015. Cited on pages 65 and 97.

[233] P. Fazekas and E. Tosatti. Electrical, structural and magnetic properties of pure and doped 1T-TaS 2 . Philosophical Magazine B, 39(3):229-244, 1979. Cited on page 65.

[234] P. Darancet, A. J. Millis, and C. A. Marianetti. Three-dimensional metallic and twodimensional insulating behavior in octahedral tantalum dichalcogenides. Physical Review B, 90(4):045134, 25, 2014. Cited on pages 65 and 96.

[235] K. T. Law and P. A. Lee. 1T-TaS 2 as a quantum spin liquid. Proceedings of the National Academy of Sciences, 114(27):6996-7000, 3, 2017. Cited on page 65.

[236] M. Klanjšek, A. Zorko, R. Žitko, J. Mravlje, Z. Jagličić, P. K. Biswas, P. Prelovšek, D. Mihailovic, and D. Arčon. A high-temperature quantum spin liquid with polaron spins. Nature Physics, 13(11):1130-1134, 2017. Cited on page 65.

[237] B. Dardel, M. Grioni, D. Malterre, P. Weibel, Y. Baer, and F. Lévy. Spectroscopic signatures of phase transitions in a charge-density-wave system: $1 T-\mathrm{TaS}_{2}$. Physical Review B, 46(12):7407-7412, 15, 1992. Cited on page 65. 
[238] T. Ishiguro and H. Sato. High-resolution electron microscopy of discommensuration in the nearly commensurate phase on warming of $1 T-\mathrm{TaS}_{2}$. Physical Review B, 52(2):759-765, 1, 1995. Cited on page 66.

[239] L. Le Guyader, T. Chase, A. H. Reid, R. K. Li, D. Svetin, X. Shen, T. Vecchione, X. J. Wang, D. Mihailovic, and H. A. Dürr. Stacking order dynamics in the quasi-two-dimensional dichalcogenide $1 T-\mathrm{TaS}_{2}$ probed with $\mathrm{MeV}$ ultrafast electron diffraction. Structural Dynamics, 4(4):044020, 2017. Cited on page 66.

[240] P. Kusar, T. Mertelj, V. V. Kabanov, J.-H. Chu, I. R. Fisher, H. Berger, L. Forró, and D. Mihailovic. Anharmonic order-parameter oscillations and lattice coupling in strongly driven 1T-TaS2 and TbTe3 charge-density-wave compounds: A multiple-pulse femtosecond laser spectroscopy study. Physical Review B, 83(3):035104, 10, 2011. Cited on page 66.

[241] J. Li, J. Li, K. Sun, L. Wu, H. Huang, R. Li, J. Yang, X. Wang, H. Luo, R. J. Cava, I. K. Robinson, Y. Zhu, and J. Tao. Ultrafast decoupling of atomic sublattices in a chargedensity-wave material. arXiv:1903.09911, 2019. Cited on pages 68 and 77.

[242] M. R. Otto, J.-H. Pöhls, L. P. R. de Cotret, M. J. Stern, M. Sutton, and B. J. Siwick. Ultrafast signatures of exciton-phonon coupling in $\mathrm{TiSe}_{2}$. arXiv:1912.03559 [cond-mat], 7, 2019. Cited on page 68.

[243] X. Gu and R. Yang. Phonon transport in single-layer transition metal dichalcogenides: A first-principles study. Applied Physics Letters, 105(13):131903, 29, 2014. Cited on page 68.

[244] M. Mohr, J. Maultzsch, E. Dobardžić, S. Reich, I. Milošević, M. Damnjanović, A. Bosak, M. Krisch, and C. Thomsen. Phonon dispersion of graphite by inelastic x-ray scattering. Physical Review B, 76(3):035439, 30, 2007. Cited on page 69.

[245] Note that the different behavior of these inequivalent peaks is unrelated to a potential decoupling of atomic sublattices, as reported in Ref. [Li, 2019] based on different groups of peaks. In fact, the diffraction signal is inherently mostly affected by the sulfur atoms due to the much larger atomic scattering factors at $100 \mathrm{eV}$ energy. Pat. Cited on page 69.

[246] M. Eichberger. Probing Structural Dynamics in Dichalcogenides with Ultrashort Electron Pulses. PhD thesis. Universität Konstanz, 2014. Cited on page 75.

[247] M. Ligges, I. Avigo, D. Golež, H. U. R. Strand, Y. Beyazit, K. Hanff, F. Diekmann, L. Stojchevska, M. Kalläne, P. Zhou, K. Rossnagel, M. Eckstein, P. Werner, and U. Bovensiepen. Ultrafast Doublon Dynamics in Photoexcited $1 T-\mathrm{TaS}_{2}$. Physical Review Letters, 120(16):166401, 18, 2018. Cited on page 76. 
[248] M. Trigo, M. Fuchs, J. Chen, M. P. Jiang, M. Cammarata, S. Fahy, D. M. Fritz, K. Gaffney, S. Ghimire, A. Higginbotham, S. L. Johnson, M. E. Kozina, J. Larsson, H. Lemke, A. M. Lindenberg, G. Ndabashimiye, F. Quirin, K. Sokolowski-Tinten, C. Uher, G. Wang, J. S. Wark, D. Zhu, and D. A. Reis. Fourier-transform inelastic X-ray scattering from time- and momentum-dependent phonon-phonon correlations. Nature Physics, 9(12):790-794, 2013. Cited on page 76.

[249] T. Aslanyan, T. Shigenari, and K. Abe. Debye-Waller factors for incommensurate structures. Journal of Physics: Condensed Matter, 10(21). Publisher: IOP Publishing:4565, 1998. Cited on page 77.

[250] P. A. Lee, T. M. Rice, and P. W. Anderson. Fluctuation Effects at a Peierls Transition. Physical Review Letters, 31(7):462-465, 13, 1973. Cited on page 77.

[251] R. H. McKenzie and J. W. Wilkins. Effect of Lattice Zero-Point Motion on Electronic Properties of the Peierls-Fröhlich State. Physical Review Letters, 69(7):1085-1088, 17, 1992. Cited on page 77.

[252] L. Degiorgi, G. Grüner, K. Kim, R. H. McKenzie, and P. Wachter. Optical probing of thermal lattice fluctuations in charge-density-wave condensates. Physical Review B, 49(20):1475414757, 15, 1994. Cited on page 77.

[253] H. Monien. Exact Results for the Crossover from Gaussian to Non-Gaussian Order Parameter Fluctuations in Quasi-One-Dimensional Electronic Systems. Physical Review Letters, 87(12):126402, 31, 2001. Cited on page 77.

[254] M. J. Vasile, D. A. Grigg, J. E. Griffith, E. A. Fitzgerald, and P. E. Russell. Scanning probe tips formed by focused ion beams. Review of Scientific Instruments, 62(9):2167-2171, 1991. Cited on page 89.

[255] C. Lee, S. Tsujino, and R. J. D. Miller. Transmission low-energy electron diffraction using double-gated single nanotip field emitter. Applied Physics Letters, 113(1):013505, 2, 2018. Cited on page 92.

[256] J. K. Gansel, M. Thiel, M. S. Rill, M. Decker, K. Bade, V. Saile, G. v. Freymann, S. Linden, and M. Wegener. Gold Helix Photonic Metamaterial as Broadband Circular Polarizer. Science, 325(5947). Publisher: American Association for the Advancement of Science Section: Report:1513-1515, 18, 2009. Cited on page 93. 
[257] M. Deubel, G. von Freymann, M. Wegener, S. Pereira, K. Busch, and C. M. Soukoulis. Direct laser writing of three-dimensional photonic-crystal templates for telecommunications. Nature Materials, 3(7):444-447, 2004. Cited on page 93.

[258] T. van Oudheusden, E. F. de Jong, S. B. van der Geer, W. P. E. M. O. 't Root, O. J. Luiten, and B. J. Siwick. Electron source concept for single-shot sub-100 fs electron diffraction in the $100 \mathrm{keV}$ range. Journal of Applied Physics, 102(9):093501, 2007. Cited on page 94.

[259] R. P. Chatelain, V. R. Morrison, C. Godbout, and B. J. Siwick. Ultrafast electron diffraction with radio-frequency compressed electron pulses. Applied Physics Letters, 101(8):081901, 20, 2012. Cited on page 94.

[260] B. J. Siwick. http://www.physics.mcgill.ca/siwicklab/hardware.htm. 2020. Cited on page 94.

[261] L. Perfetti, P. A. Loukakos, M. Lisowski, U. Bovensiepen, H. Eisaki, and M. Wolf. Ultrafast Electron Relaxation in Superconducting $\mathrm{Bi}_{2} \mathrm{Sr}_{2} \mathrm{CaCu}_{2} \mathrm{O}_{8}+$ delta by Time-Resolved Photoelectron Spectroscopy. Physical Review Letters, 99(19):197001, 9, 2007. Cited on page 96.

[262] Z. Tao, T.-R. T. Han, and C.-Y. Ruan. Anisotropic electron-phonon coupling investigated by ultrafast electron crystallography: Three-temperature model. Physical Review B, 87(23), 20, 2013. Cited on page 96.

[263] L. Waldecker, R. Bertoni, R. Ernstorfer, and J. Vorberger. Electron-Phonon Coupling and Energy Flow in a Simple Metal beyond the Two-Temperature Approximation. Physical Review X, 6(2), 6, 2016. Cited on page 96.

[264] J.-D. Su, A. R. Sandy, J. Mohanty, O. G. Shpyrko, and M. Sutton. Collective pinning dynamics of charge-density waves in $1 T-\mathrm{TaS}_{2}$. Physical Review B, 86(20), 5, 2012. Cited on page 96.

[265] X. M. Chen, A. J. Miller, C. Nugroho, G. A. de la Peña, Y. I. Joe, A. Kogar, J. D. Brock, J. Geck, G. J. MacDougall, S. L. Cooper, E. Fradkin, D. J. Van Harlingen, and P. Abbamonte. Influence of Ti doping on the incommensurate charge density wave in $1 T-\mathrm{TaS}_{2}$. Physical Review B, 91(24):245113, 8, 2015. Cited on page 96.

[266] S. Uchida, K. Tanabe, and S. Tanaka. Nonlinear conduction in two-dimensional CDW system: 1T-TaS 2 . Solid State Communications, 27(6). Publisher: Elsevier:637-640, 1978. Cited on page 96.

[267] L. Harnau, S. Dietrich, G. Gompper, and M. Schick. Soft Matter. Publisher: Wiley-VCH Berlin, 2007. Cited on page 97. 
[268] H. Dai and C. M. Lieber. Solid-hexatic-liquid phases in two-dimensional charge-density waves. Physical Review Letters, 69(10):1576-1579, 7, 1992. Cited on page 97.

[269] I. R. Peterson and V. M. Kaganer. Diffraction line profile of a two-dimensional hexatic. Physical Review Letters, 73(1):102-105, 4, 1994. Cited on page 97.

[270] K. Sugawara, Y. Nakata, R. Shimizu, P. Han, T. Hitosugi, T. Sato, and T. Takahashi. Unconventional charge-density-wave transition in monolayer 1 T-TiSe2. ACS nano, 10(1). Publisher: ACS Publications:1341-1345, 2016. Cited on page 97.

[271] Y. Chen, W. Ruan, M. Wu, S. Tang, H. Ryu, H.-Z. Tsai, R. Lee, S. Kahn, F. Liou, C. Jia, O. R. Albertini, H. Xiong, T. Jia, Z. Liu, J. A. Sobota, A. Y. Liu, J. E. Moore, Z.-X. Shen, S. G. Louie, S.-K. Mo, and M. F. Crommie. Strong correlations and orbital texture in single-layer 1T-TaSe 2 . Nature Physics, 16(2):218-224, 2020. Cited on page 97.

[272] M. M. Ugeda, A. Pulkin, S. Tang, H. Ryu, Q. Wu, Y. Zhang, D. Wong, Z. Pedramrazi, A. Martín-Recio, Y. Chen, F. Wang, Z.-X. Shen, S.-K. Mo, O. V. Yazyev, and M. F. Crommie. Observation of topologically protected states at crystalline phase boundaries in single-layer WSe2. Nature Communications, 9(1), 2018. Cited on page 97.

[273] D. Mou, A. Sapkota, H.-H. Kung, V. Krapivin, Y. Wu, A. Kreyssig, X. Zhou, A. I. Goldman, G. Blumberg, R. Flint, and A. Kaminski. Discovery of an Unconventional Charge Density Wave at the Surface of K 0.9 Mo 6 O 17. Physical Review Letters, 116(19):196401, 13, 2016. Cited on page 98.

[274] N. Ru, C. L. Condron, G. Y. Margulis, K. Y. Shin, J. Laverock, S. B. Dugdale, M. F. Toney, and I. R. Fisher. Effect of chemical pressure on the charge density wave transition in rare-earth tritellurides R Te 3. Physical Review B, 77(3):035114, 14, 2008. Cited on page 98.

[275] D. H. Torchinsky, F. Mahmood, A. T. Bollinger, I. Božović, and N. Gedik. Fluctuating charge-density waves in a cuprate superconductor. Nature Materials, 12(5):387-391, 2013. Cited on page 98.

[276] C. Balseiro and L. Falicov. Superconductivity and charge-density waves. Physical Review B, 20(11). Publisher: APS:4457, 1979. Cited on page 98.

[277] S. A. Kivelson, I. P. Bindloss, E. Fradkin, V. Oganesyan, J. M. Tranquada, A. Kapitulnik, and C. Howald. How to detect fluctuating stripes in the high-temperature superconductors. Reviews of Modern Physics, 75(4):1201-1241, 8, 2003. Cited on page 98. 
[278] J. K. Kjems, L. Passell, H. Taub, J. G. Dash, and A. D. Novaco. Neutron scattering study of nitrogen adsorbed on basal-plane-oriented graphite. Physical Review B, 13(4):1446-1462, 15, 1976. Cited on page 98.

[279] M. D. Chinn and S. C. Fain. Structural Phase Transition in Epitaxial Solid Krypton Monolayers on Graphite. Physical Review Letters, 39(3):146-149, 18, 1977. Cited on page 98.

[280] C. G. Shaw, S. C. Fain, and M. D. Chinn. Observation of Orientational Ordering of Incommensurate Argon Monolayers on Graphite. Physical Review Letters, 41(14):955-957, 2, 1978. Cited on page 98.

[281] S. C. Fain, M. D. Chinn, and R. D. Diehl. Commensurate-incommensurate transition of solid krypton monolayers on graphite. Physical Review B, 21(9):4170-4172, 1, 1980. Cited on page 98.

[282] P. W. Stephens, P. Heiney, R. J. Birgeneau, and P. M. Horn. X-Ray Scattering Study of the Commensurate-Incommensurate Transition of Monolayer Krypton on Graphite. Physical Review Letters, 43(1):47-51, 2, 1979. Cited on page 98.

[283] D. E. Moncton, P. W. Stephens, R. J. Birgeneau, P. M. Horn, and G. S. Brown. Synchrotron X-Ray Study of the Commensurate-Incommensurate Transition of Monolayer Krypton on Graphite. Physical Review Letters, 46(23):1533-1536, 8, 1981. Cited on page 98.

[284] H. Shiba. The Nonregistered-Registered Phase Transition of Rare Gas Monolayers Adsorbed on Graphite. Journal of the Physical Society of Japan, 46(6). Publisher: Institute of Pure and Applied Physics, 1979. Cited on page 98.

[285] P. Bak, D. Mukamel, J. Villain, and K. Wentowska. Commensurate-incommensurate transitions in rare-gas monolayers adsorbed on graphite and in layered charge-density-wave systems. Physical Review B, 19(3):1610-1613, 1, 1979. Cited on page 98.

[286] J. Villain. Ordering in two dimensions. Proceedings of an International Conference on Ordering in Two Dimensions (Ordering in Two Dimensions. Proceedings of an International Conference on Ordering in Two Dimensions, Lake Geneva, WI, USA, 28-30 May 1980), edited by Sinha (SK Amsterdam, Netherlands: North-Holland, 1981). 1980, pp. 123-129. Cited on page 98.

[287] S. N. Coppersmith, D. S. Fisher, B. I. Halperin, P. A. Lee, and W. F. Brinkman. Dislocations and the Commensurate-Incommensurate Transition in Two Dimensions. Physical Review Letters, 46(8):549-552, 23, 1981. Cited on page 98. 
[288] E. Ustinov. Effective and accurate approach for modeling of commensurate-incommensurate transition in krypton monolayer on graphite. The Journal of chemical physics, 141(13). Publisher: American Institute of Physics:134706, 2014. Cited on page 98.

[289] M. Reiser and P. O'Shea. Theory and design of charged particle beams. Wiley Online Library, 1994.

[290] M. Born and E. Wolf. Principles of optics: electromagnetic theory of propagation, interference and diffraction of light. Elsevier, 2013.

[291] J. P. Snyder. Map projections-A working manual. US Government Printing Office, 1987. 


\section{Acknowledgements}

At the end of my work, I would like to thank the people who accompanied me on my way as a doctoral student.

In first place, I would like to thank Claus Ropers for showing me the way of the scientist, for the long years of fruitful and constructive collaboration, and for trusting the work I did.

I also thank all members of the examination committee, in particular Stefan Mathias for being the second reviewer of my work. I would like to point out that despite the global pandemic and the uncertain times, the committee was very supportive and communication was very reliable.

During the process of writing my scientific publications, I had the pleasure to work with brilliant scientists, including Sascha Schäfer, Murat Sivis, Simon Vogelgesang, Jan Gerrit Horstmann, Theo Diekmann, Gevin von Witte, Sergey Yalunin and Kai Rossnagel.

I am also grateful to those who carefully proofread my thesis: Hugo Lourenco-Martins, Bart Wit, Jan Gerrit Horstmann and Susa Thomas.

Special thanks goes to the ULEED tribe, my various office mates over the past years, the coffee break crowd and the GSR team. In times of Covid-19, it becomes even more apparent how joyful, necessary and important it is to work closely with colleagues in the workplace.

I would also like to thank the technical support that was crucial for many steps of this work. In particular, I thank Volker Radisch, Jochen Herbst, Jörg Malindretos, Thomas Lehmann, Karin Ahlborn, Bernhard Spicher, Marie Petri and the colleagues in the central workshop.

Finally, I would like to thank my family and, especially, my wife Kira who always supported me and lightened up my day in very dark times. 